\title{
Flowsheet Evaluation for the Dissolving and Neutralization of Sodium Reactor Experiment Used Nuclear Fuel
}

W. E. Daniel

E. K. Hansen

T. C. Shehee

October 2012 
SRNL-STI-2012-00279

Revision 1

\section{DISCLAIMER}

This work was prepared under an agreement with and funded by the U.S. Government. Neither the U.S. Government or its employees, nor any of its contractors, subcontractors or their employees, makes any express or implied:

1. warranty or assumes any legal liability for the accuracy, completeness, or for the use or results of such use of any information, product, or process disclosed; or

2. representation that such use or results of such use would not infringe privately owned rights; or

3. endorsement or recommendation of any specifically identified commercial product, process, or service.

Any views and opinions of authors expressed in this work do not necessarily state or reflect those of the United States Government, or its contractors, or subcontractors.

\section{Printed in the United States of America \\ Prepared for U.S. Department of Energy}


Keywords: Dissolution,

Neutralization, HMI, DR3, SRE, UNF

Retention: Permanent

\title{
Flowsheet Evaluation for the Dissolving and Neutralization of Sodium Reactor Experiment Used Nuclear Fuel
}

\author{
W. E. Daniel \\ E. K. Hansen \\ T. C. Shehee
}

October 2012

Savannah River National Laboratory Savannah River Nuclear Solutions, LLC Aiken, SC 29808

Prepared for the U.S. Department of Energy under contract number DE-AC09-08SR22470. 


\section{REVIEWS AND APPROVALS}

AUTHORS:

W. E. Daniel, Process Technology Programs

Date

E. K. Hansen, Engineering and Process Development

Date

T. C. Shehee, Separations \& Actinide Science Programs

Date

TECHNICAL REVIEW:

M. C. Thompson, Separations \& Actinide Science Programs

Date

F. Fondeur, Separations \& Actinide Science Programs

Date

APPROVAL:

S. D. Fink, Manager

Date

Separations \& Actinide Science Programs

S. L. Marra, Manager

Date

Environmental \& Chemical Process Technology Research Programs

W. G. Dyer, Manager

Date

H-Canyon Engineering 
SRNL-STI-2012-00279

Revision 1

\section{PREFACE OR ACKNOWLEDGEMENTS}

Thanks to Bill Clifton and Ric Castles for providing data and drawings of the various used nuclear fuel assemblies. 


\section{EXECUTIVE SUMMARY}

This report includes the literature review, hydrogen off-gas calculations, and hydrogen generation tests to determine that H-Canyon can safely dissolve the Sodium Reactor Experiment (SRE; thorium fuel), Ford Nuclear Reactor (FNR; aluminum alloy fuel), and Denmark Reactor (DR-3; silicide fuel, aluminum alloy fuel, and aluminum oxide fuel) assemblies in the L-Bundles with respect to the hydrogen levels in the projected peak off-gas rates. This is provided that the number of L-Bundles charged to the dissolver is controlled. Examination of SRE dissolution for potential issues has aided in predicting the optimal batching scenario.

As demonstrated in prior work on the dissolution of the University of Missouri Research Reactor (MURR) assemblies, this work relies on the literature composite lower flammability limits (LFL) for $\mathrm{H}_{2}$ in an air/NO/ $\mathrm{N}_{2} \mathrm{O}$ mixture and pilot-scale studies on the dissolution of Savannah River Site (SRS) fuels. How the various used nuclear fuels are charged to the dissolver, the depth of the dissolver solution, and the concentrations of nitric acid and catalysts (mercury and fluoride) will control the dissolution rate during the initial stage of the dissolution cycle. The concentration of catalysts (mercury and fluoride), the nitric acid concentration, and the dissolved metals concentrations (aluminum, thorium) impact the off-gas generation rates and thus the hydrogen generated during dissolution. The calculations detailed in this report demonstrate that the FNR, SRE, and DR-3 used nuclear fuel (UNF) are bounded by MURR UNF and may be charged using the controls outlined for MURR dissolution in a prior report.

The physical property measurements and flow calculations on the dissolved SRE, FNR, and DR-3 UNF simulants indicate that the material should flow down the waste header in Building 221- $\mathrm{H}$. For conservativeness, the analysis assumed the lines were partially full of fluid with no air bubble entrapment. The flow calculations show that the initial section of the waste lines leaving Building 221- $\mathrm{H}$ is limited to $27.2 \mathrm{gpm}$ since the waste header has no vertical drop in the piping. The flow calculations showed that the secondary sections of waste lines provide a flow of at least $45.3 \mathrm{gpm}$. These calculated flow rates are for dirty piping. For clean piping, the calculated flow rates would be slightly larger. When the various sections of pipe are completely filled with fluid the calculated minimum flow rate increases to $58.4 \mathrm{gpm}$. This indicates that the hydrostatic head has increased relative to the pressure drop associated with the additional piping. The calculations show that the waste lines leaving Building 221- $\mathrm{H}$ could handle the 25 to $30 \mathrm{gpm}$ flow that the steam jets provide. In reality, the waste lines are most likely not at full pipe flow.

It is recommended that Waste Header \#1, 4, or 3 (transfer lines WF1100, WF1101, or WF1102 respectively) be used if there is a potential for the fluid to have a Bingham Plastic yield stress of larger than $1 \mathrm{~Pa}$ such as those of the 12,000L dissolver batch fluids. If Waste Header \#2 (WF1103) is used for such fluids, the fluid will backup in to the header in Building 221-H. The 10-inch header associated with WH \#2 may backup and provide the necessary head for $25 \mathrm{gpm}$, but this was not analyzed.

The deposition velocity was calculated between 3.5 to $4.1 \mathrm{ft} / \mathrm{s}$. Given a discharge rate of $25 \mathrm{gpm}$, only the steepest transfer lines have a potential to mitigate settling of solids. Intermittent flushing with inhibited water is recommended in minimizing undissolved solids buildup at the maximum achievable flow rate with a minimum of at least 3 waste line volumes or an acceptable drop rate in the waste header liquid level is observed. 


\section{TABLE OF CONTENTS}

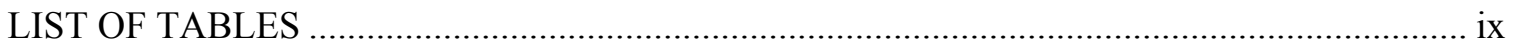

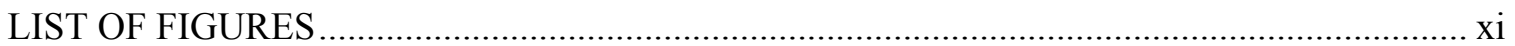

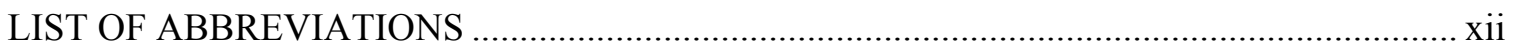

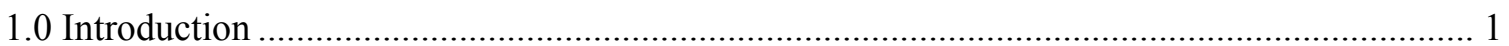

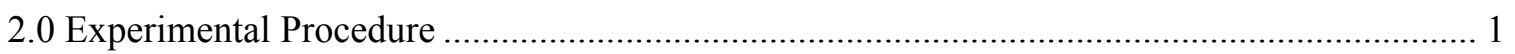

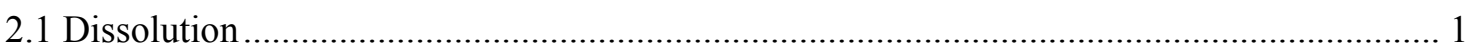

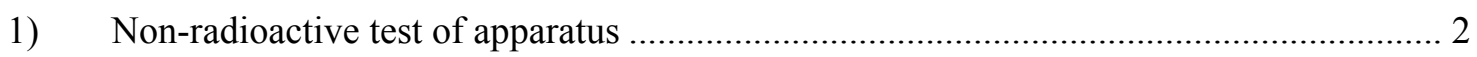

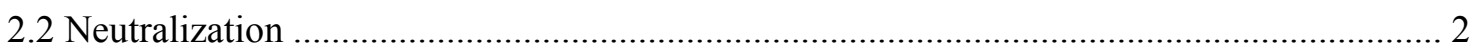

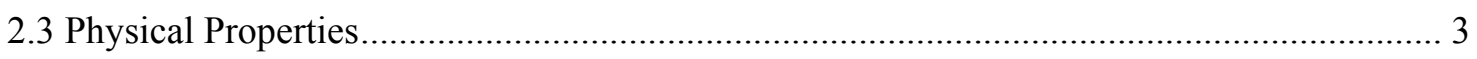

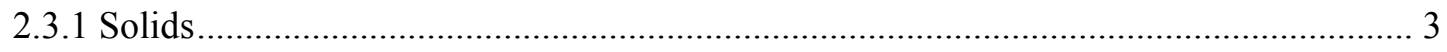

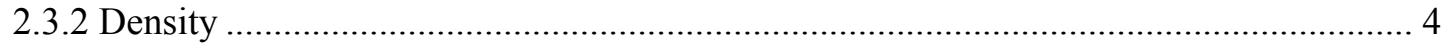

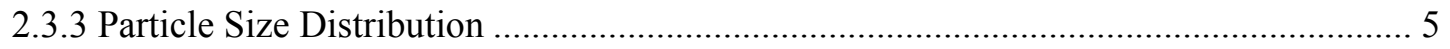

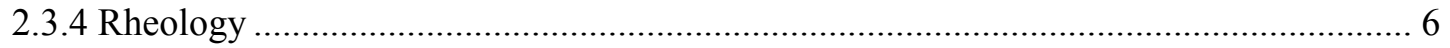

2.3.5 Method to Determine Average Velocity and Volumetric Flow Rate ............................. 7

2.3.6 Methods to Determine Non-Newtonian pipe frictional losses ..................................... 9

2.3.7 Impact of Partial Pipe Fill on Solids Suspension ...................................................... 10

2.3.8 Determining Deposition Velocity and Sediment Transport ......................................... 12

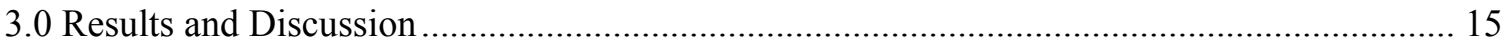

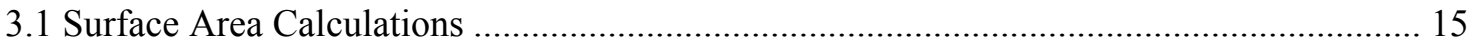

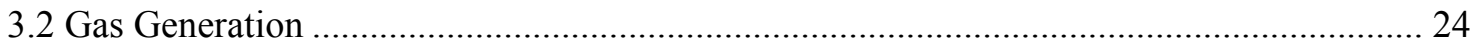

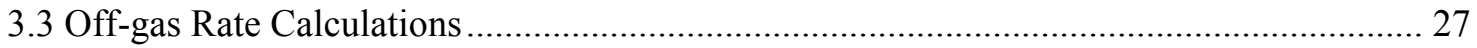

3.3.1 Off-gas Rate Calculation Assumptions and Conditions ................................................. 27

3.3.2 Off-gas Rate Values for Dissolver Charging Scenarios .............................................. 35

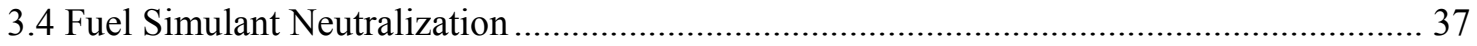

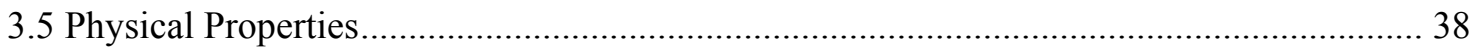

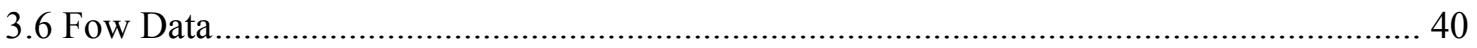

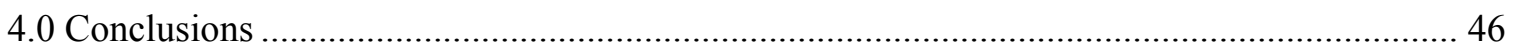

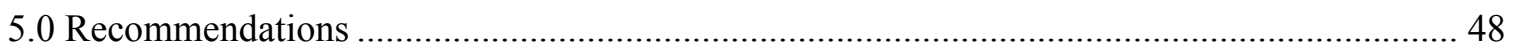

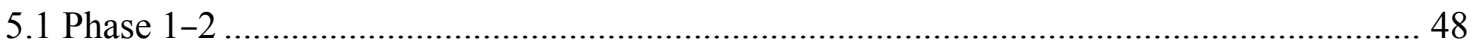

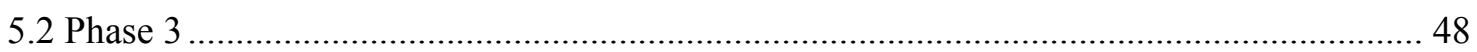

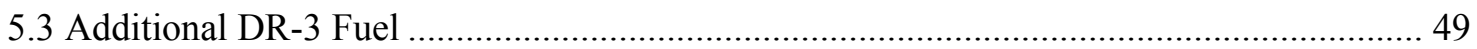

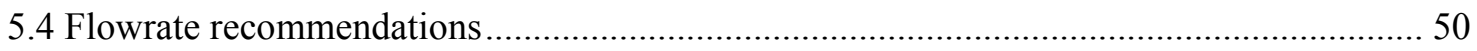

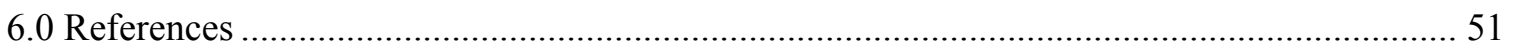


A.1. Disassembly L-Area Bundling Tube Surface Area Calculations. 54

A.2. Sodium Reactor Experiment (SRE) Surface Area Calculations 63

A.3. Hahn-Meitner-Institut (HMI) Surface Area Calculations 69

A.3.1. Standard HMI Assembly Surface Area Calculations 69

A.3.2. Control HMI Assembly Surface Area Calculations 72

A.4. DR-3 (Denmark Reactor) Surface Area Calculations .................................................. 76

A.4.1. DR-3-1 (Denmark Reactor) Surface Area Calculations …………......................... 76

A.4.2. DR-3-2 (Denmark Reactor) Surface Area Calculations ........................................ 82 


\section{LIST OF TABLES}

Table 2-1. Dissolution schemes used to determine bounding $\mathrm{H}_{2}$ gas generation rates. 2

Table 2-2. Uranium, Thorium and Aluminum content in SRE, DR-3, and FNR UNF mixed dissolver solutions. ${ }^{1}$

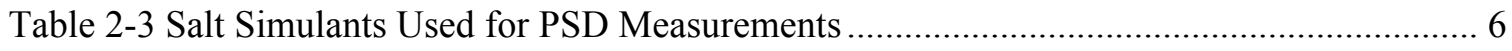

Table 2-4. Sludge Flow Curve Profile Using MV1 Geometry .................................................. 7

Table 2-5. Complete Elevation, Piping Run, Elbows, Entrance and Exit Data for Waste Transfer Line between Building 221-H to HPP\#5 and HPP\#6 14

Table 2-6. Initial Elevation, Piping Run, Elbows, Entrance for Waste Transfer Exiting Building $221-\mathrm{H}$ 14

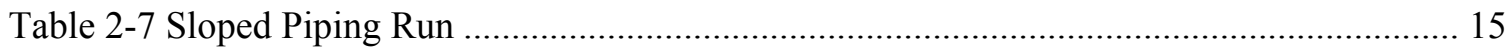

Table 3-1. L-Bundle and UNF Assemblies Total Surface Areas per Immersion Height ............. 16

Table 3-2. L-Bundle and UNF Assemblies Surface Areas per Immersion Height for Off-gas Calculations. 20

Table 3-3. Initial results of dissolution studies 26

Table 3-4. $\mathrm{H}_{2}$ Lower Flammability Limit from Scott and Dyer Data........................................ 28

Table 3-5. Off-gas Composition Values for Dissolving Thorium Metal.................................... 29

Table 3-6. Peak Off-gas Generation Rate for Thorium Dissolving Thorium Metal.................... 29

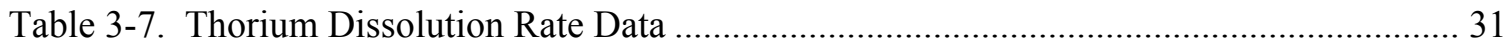

Table 3-8. Thorium Dissolution Rate Fits as function of $\mathrm{HNO}_{3}$ and thorium Concentrations..... 34

Table 3-9. Predicted Thorium Dissolution Rates at 7 and $10 \mathrm{M} \mathrm{HNO}_{3}$ and $0.05 \mathrm{M} \mathrm{F}$................ 34

Table 3-10. Example Off-Gas Rates for Dissolver Batching Scenario with 4 SRE L-Bundles... 36

Table 3-11 Undissolved Solids Density Determination Using Concentrated 14,000 L Dissolver Fluids. 38

Table 3-12 Particle Size Distribution, Percentiles and Mean Values 39

Table 3-13. Energy Equations for Velocity Determination for Waste Transfer Lines Between Building 221-H and HPP

Table 3-14. Energy Equations for Velocity Determination for Initial Section of the Waste Transfer Lines Leaving Building 221-H with Slope of 0.005.

Table 3-15. Average Velocity, Reynolds Number, and Flow rate for Waste Transfer Lines Between Building 221-H and HPP 
Table 3-16. Average Velocity, Reynolds Number, and Flow rate for Initial Section of the Waste Transfer Lines Leaving Building 221-H With Slope of 0.005.

Table 3-17 Deposition, Dimensionless Grain Diameter, Fill Ratio, Velocity and Reynolds Number For the Minimum and Maximum Pipe Slopes at 25 GPM.

Table 3-18 Maximum Flow Using The Most non-Newtonian Solution, 12000 L 4FS19D-0.8M 43

Table 3-19. Supernate, Slurry, and Undissolved Density and Solids Fraction Data, 14,000 L and 12,000 L Dissolver Batch Fluids.

Table 3-20. Rheological Data, 14,000 L and 12,000 L Dissolver Batch Fluids.

Table A-1. Disassembly L-Area Bundling Tube Parts and Dimensions .................................... 55

Table A-2. Inner and Outer Surface Area Calculation for DABT or L-Bundle ........................... 56

Table A-3. Clearances between L-Bundle and SRE, HMI, and DR-3 Assemblies ..................... 59

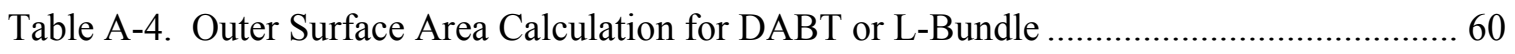

Table A-5. Sodium Reactor Experiment (SRE) Shipping Can Parts and Dimensions.................. 63

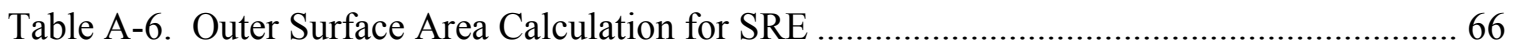

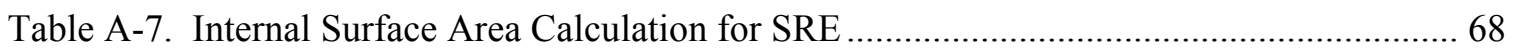

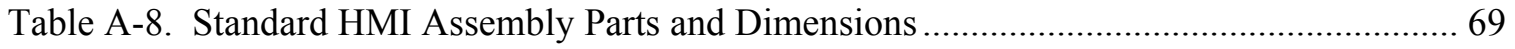

Table A-9. Surface Area Calculation for Standard HMI Assembly .......................................... 71

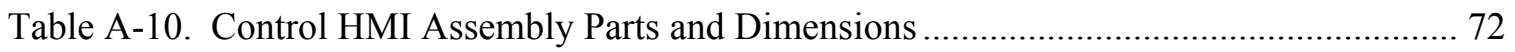

Table A-11. Surface Area Calculation for Control HMI Assembly ............................................. 74

Table A-12. Denmark Reactor DR-3-1 Assembly Parts and Dimensions.................................... 80

Table A-13. Inner and Outer Surface Area Calculation for DR-3-1 Assembly .......................... 81

Table A-14. Inner and Outer Surface Area Calculation for DR-3-2 Assembly .......................... 84 


\section{LIST OF FIGURES}

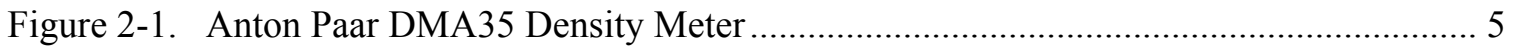

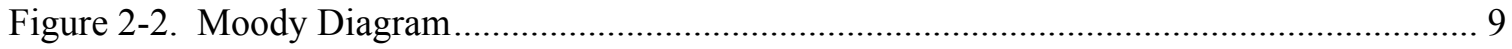

Figure 2-3. Partial Pipe Fill To Determine The Hydraulic Radius for a Given Fill Height ......... 11

Figure 3-1. L-Bundle and Various UNF Assemblies Total Surface Areas per Immersion Height

Figure 3-2. Dissolution apparatus showing the dark brown gas generated during the dissolution

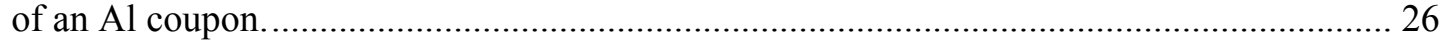

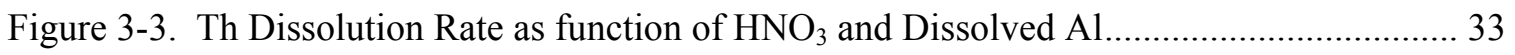

Figure 3-4. Th Dissolution Rate as function of $\mathrm{HNO}_{3}$ and Th Concentrations ........................... 33

Figure 3-5. Th Dissolution Rate at 7 and $10 \mathrm{M} \mathrm{HNO}_{3}, 0.05 \mathrm{M} \mathrm{F}$ as Function of Th Concentration

Figure 3-6. Neutralization of simulated dissolver batch. A) after addition of a few $\mathrm{mL} 50 \mathrm{wt} \%$ $\mathrm{NaOH}$ showing initial precipitation; B) neutralization at $\mathrm{pH} 4.5$; C) neutralization at $\mathrm{pH} 7$;

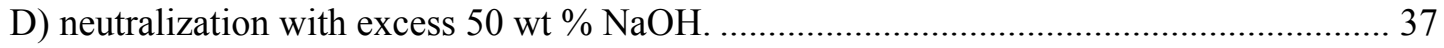

Figure 3-7 Flow Curves for 14,000 and 12,000 L 4F5S19D Slurries ......................................... 39

Figure 3-8 Particle Size Distribution of 12,000 L Dissolver Batch Fluids.................................. 40

Figure A-1. Sketch of Disassembly L-Area Bundling Tube (DABT) or L-Bundle ..................... 54

Figure A-2. Sketch of Sodium Reactor Experiment (SRE) Shipping Can ................................. 64

Figure A-3. Sketch of Cross-section of Sodium Reactor Experiment (SRE) Shipping Can ........ 65

Figure A-4. Sketch of Lifting Bail of Sodium Reactor Experiment (SRE) Shipping Can ........... 65

Figure A-5. Sketch of Standard HMI Assembly with Cropped Sections Cross Hatched............. 70

Figure A-6. Sketch of Standard HMI Assembly with Side Plate Removed ................................ 70

Figure A-7. Sketch of Control HMI Assembly with Cropped Sections Cross Hatched............... 73

Figure A-8. Sketch of End of Control HMI Assembly with Cropped Sections Cross Hatched ... 73

Figure A-9. Sketch of Control HMI Assembly with Front Side Plate Removed ......................... 73

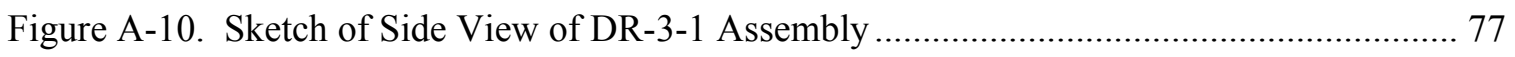

Figure A-11. Sketch of Top Cross-section of DR-3-1 Assembly ............................................... 78

Figure A-12. Sketch of Side Cross-section of DR-3-1 Assembly ….......................................... 79 
Figure A-13. Sketch of Top and Bottom Cup Plates of DR-3-1 Assembly

Figure A-14. Sketch of Side View of DR-3-2 Assembly ...

Figure A-15. Sketch of Side Cross-section of DR-3-2 Assembly 83

\section{LIST OF ABBREVIATIONS}

$\begin{array}{ll}\text { DABT } & \text { Disassembly L-Area Bundling Tube or L-Bundle } \\ \text { DI } & \text { Distilled and deionized } \\ \text { DR-3 } & \text { Denmark Reactor } \\ \text { DU } & \text { Depleted Uranium } \\ \text { FNR } & \text { Ford Nuclear Reactor } \\ \text { HA/LU } & \text { High Aluminum / Low Uranium } \\ \text { HMI } & \text { Hahn-Meitner-Institute } \\ \text { HPP } & \text { H-Area Pump Pit } \\ \text { LFL } & \text { Lower Flammability Limit } \\ \text { MURR } & \text { University of Missouri Research Reactor } \\ \text { PSD } & \text { particle size distribution } \\ \text { scfm } & \text { standard cubic feet per minute } \\ \text { SG } & \text { Specific gravity } \\ \text { SRE } & \text { Sodium Reactor Experiment } \\ \text { SRNL } & \text { Savannah River National Laboratory } \\ \text { STDEV } & \text { Standard Deviation } \\ \text { UDS } & \text { undissolved solids } \\ \text { UNF } & \text { used nuclear fuel } \\ \text { WH } & \text { Waste Header }\end{array}$




\subsection{Introduction}

The H-Canyon processing of Sodium Reactor Experiment (SRE) ${ }^{1}$ Used Nuclear Fuel (UNF) is to begin in 2012. The SRE fuel is currently being stored in L-Basin. SRE is a thorium/uranium alloy that is sealed in aluminum cans in a Disassembly L-Area Bundling Tube (DABT) also known as an L-Bundle. This fuel is identified as "vulnerable" and needs a timely disposition. The SRE fuel will be dissolved in HCanyon along with a High Aluminum/Low Uranium-235 (HA/LU) UNF. Once dissolved, depleted uranium will be added to the dissolver solution to reduce the ${ }^{235} \mathrm{U}$ enrichment in the material if required to meet liquid waste requirements.

The candidate HA/LU fuels are the FNR and DR-3 fuels. The DR-3 UNF originated from the Ris $\varnothing$ National Laboratory in Roskilde, Denmark. The majority of this fuel is an aluminum clad silicide type fuel where the fuel "meat" is $\mathrm{U}_{3} \mathrm{Si}_{2}-\mathrm{Al}$ alloy. Dissolution of silicide fuel should be such that the [Si] remains well below the threshold concentration of $0.1 \mathrm{M}$ silicon. This concentration avoids formation of gelatinous silicic acid. ${ }^{2}$ Another DR-3 UNF, recently identified for processing, is either $\mathrm{U}_{-} \mathrm{Al}_{\mathrm{x}}$ or $\mathrm{U}_{3} \mathrm{O}_{8}-\mathrm{Al}$ (cermet). Hahn-Meitner-Institute (HMI) UNF was an early candidate HA/LU fuels. This fuel was included in the surface area calculations, but will not be dissolved at this time.

SRNL was tasked with determining through literature search and laboratory experimentation the flowsheet parameters necessary to safely and effectively dissolve, process, and neutralize the SRE and HA/LU UNF in H-Canyon. The flowsheet evaluation defined the number of SRE and HA/LU bundles that can safely be charged to the dissolver batch without exceeding the hydrogen lower flammability limit (LFL). SRNL evaluated issues pertaining to the dissolution of the various fuels together and provided guidance for charging either the HA/LU material to the dissolved SRE solution, or vice-versa. SRNL also provided limits and controls for ensuring the neutralized solution flows properly down the waste header after neutralization. Determination of these limits and controls have been established from testing the rheological properties of the mixtures derived from the addition of $50 \mathrm{wt} \% \mathrm{NaOH}$ solution to several dissolver simulant solutions.

\subsection{Experimental Procedure}

\subsection{Dissolution}

Thorium (Th) and aluminum (Al) metal were dissolved under a variety of conditions in nitric acid (Table 2-1) to measure the gas generated during dissolution as well as dissolution time. The experimental configuration for these studies was modeled after the apparatus in a recent report ${ }^{3}$, where dissolver solution is added into a glass reaction vessel, placed onto a hot plate-stirrer, and heated to $50-100{ }^{\circ} \mathrm{C}$. A $75 \mathrm{~mL}$ dissolver solution volume was selected for dissolutions based on the available mass of Th metal, the calculated total gas production, and the minimum solution needed to completely cover the one inch square thorium coupon. The vessel was purged with nitrogen or argon to remove air prior to starting dissolution by passing the purge gas through the dissolver, condenser and sample bulb. The gas port automatically closes when the gas line is removed which precludes the introduction of $\mathrm{O}_{2}$ to the system. The reaction dissolver solution was heated to $99 \pm 1{ }^{\circ} \mathrm{C}$, at which time a coupon of $99.5 \mathrm{wt} \% \mathrm{Th}$ metal of known weight and physical dimensions was immersed into the dissolver solution using a glass basket. The sample remained immersed until the Th was completely dissolved. Off-gas volume generated during the dissolution was measured and samples collected. The dissolving system utilizes Tedlar ${ }^{\circledR}$ bags to collect the off-gas and $40 \mathrm{~mL}$ glass sample bulbs to collect subsamples for analysis. The volume of gas collected in each bag was determined by water displacement with an accuracy of $\pm 5 \mathrm{~mL}$. Off-gas collected during the experiments was analyzed by gas chromatography to determine composition. 
Table 2-1. Dissolution schemes used to determine bounding $\mathrm{H}_{2}$ gas generation rates.

\begin{tabular}{|c|c|c|c|c|c|c|}
\hline Dissolution & \multicolumn{2}{|c|}{ Metal foil used } & {$\left[\mathrm{HNO}_{3}\right]_{\text {Initial }}$} & {$\left[\mathbf{F}^{-}\right]$} & {$\left[\mathrm{Hg}^{2+}\right]$} & {$[\mathrm{Al}]_{\text {Initial }}{ }^{2}$} \\
\hline $1^{1}$ & - & $\mathrm{Al}$ & 10 & - & 0.02 & 0.3 \\
\hline 1-A & - & $\mathrm{Al}$ & 10 & - & 0.002 & 0.3 \\
\hline 2 & $\mathrm{Th}$ & - & 4 & 0.05 & - & - \\
\hline 3 & $\mathrm{Th}$ & - & 7 & 0.05 & - & - \\
\hline 4 & $\mathrm{Th}$ & - & 10 & 0.05 & - & - \\
\hline 5 & Th & - & 7 & 0.05 & - & 0.3 \\
\hline $3-A^{3}$ & $\mathrm{Th}^{4+}{ }_{(\mathrm{aq})}$ & $\mathrm{Al}$ & 7 & 0.05 & 0.002 & - \\
\hline $4-A^{3}$ & $\mathrm{Th}^{4+}{ }_{(\mathrm{aq})}$ & $\mathrm{Al}$ & 10 & 0.05 & 0.002 & - \\
\hline $5-A^{3}$ & $\mathrm{Th}^{4+}{ }_{(\mathrm{aq})}$ & $\mathrm{Al}$ & 7 & 0.05 & 0.002 & 0.3 \\
\hline 5-B & Th & - & 7 & 0.01 & - & 0.3 \\
\hline 6 & $\mathrm{Th}$ & - & 10 & 0.05 & - & 0.3 \\
\hline 7 & $\mathrm{Th}$ & $\mathrm{Al}$ & 10 & 0.05 & - & 0.3 \\
\hline 8 & $\mathrm{Th}$ & $\mathrm{Al}$ & 10 & 0.05 & 0.002 & 0.3 \\
\hline $9^{4}$ & $\mathrm{Th}$ & $\mathrm{Al}$ & 10 & 0.05 & 0.002 & 0.3 \\
\hline $10^{4}$ & $\mathrm{Th}$ & - & 7 & 0.05 & - & 0.3 \\
\hline
\end{tabular}

1) Non-radioactive test of apparatus

2) Represents the concentration present after dissolution of FNR and part of SRE bundle

3) Dissolver solution is from Th dissolutions 3, 4 and 5. Hg added to aid in dissolution of Al.

4) Duplicate Measurement

\subsection{Neutralization}

SRNL personnel prepared four simulant solution stocks representing the expected composition of SRE UNF and multiple dissolver batch blends. Simulant compositions are shown in Table 2-2. These concentrations were selected from the compositions of the fuels expected to be processed at the same time, the expected order of dissolver additions, and the typical volume used in the dissolver. ${ }^{2}$ All simulants included depleted uranium (DU) at concentrations that represent a dissolved fuel with added DU such that the isotopic ratio was reduced to $5 \mathrm{wt} \%$. This provides a bounding scenario should it be determined that a further down blending is required. If necessary, $\mathrm{DU}$ is available in the form of $\mathrm{UO}_{3}$ powder, with a ${ }^{235} \mathrm{U}$ concentration of approximately $0.2 \mathrm{wt} \%$, dissolved to several hundred $\mathrm{g} / \mathrm{L} \mathrm{U}$ as needed and added to the dissolver solution. All simulants also included gadolinium $(\mathrm{Gd})$ at $0.5 \mathrm{~g} / \mathrm{L}$ to represent the $\mathrm{Gd}$ added as a neutron poison. Mercury has been added since it was required to aid in the dissolution of the HA/LU fuel and the L-Bundles.

Precipitation and neutralization experiments were performed on each simulant shown in Table 2-2. The acidic solution was neutralized to the $\mathrm{pH}$ where the first solids precipitate and did not redissolve, expected to be at a $\mathrm{pH}$ of $\sim 4.5$; to $\mathrm{pH} 7$; to $0.8 \mathrm{M}$ free hydroxide; and to $1.2 \mathrm{M}$ free hydroxide. The neutralization uses $50 \mathrm{wt} \% \mathrm{NaOH}$, calculated to achieve the above neutralization points and dispensed from a $50 \mathrm{~mL}$ burette. The quantity of $50 \mathrm{wt} \% \mathrm{NaOH}$ used to achieve excess free hydroxide included any consumed by the aluminum present in the system. To monitor the first two $\mathrm{pH}$ points, EMD brand $\mathrm{pH} 0-14$ indicator strips were used. For samples that approximate an evaporated dissolver solution, the simulant was heated to pre-concentrate each solution to $85 \%$ of the original volume (equivalent of a change in dissolver volume of $14000 \mathrm{~L}$ to $12000 \mathrm{~L}$ due to evaporation). This simulant was then neutralized as outlined above. Samples of the neutralization to the 0.8 and $1.2 \mathrm{M}$ excess were collected for rheology and density measurements. 
SRNL-STI-2012-00279

Revision 1

Table 2-2. Uranium, Thorium and Aluminum content in SRE, DR-3, and FNR UNF mixed dissolver solutions. ${ }^{1}$

\begin{tabular}{|c|c|c|c|c|c|c|}
\hline Fuel simulants & $\begin{array}{c}\text { Final [U] after } \\
\text { DU Addition } \\
\text { (g/L) }\end{array}$ & $\begin{array}{c}\text { [Th] } \\
\text { (g/L) }\end{array}$ & $\begin{array}{c}\text { [Al] } \\
\text { (g/L) }\end{array}$ & [U] (M) & [Th] (M) & [Al] (M) \\
\hline $\begin{array}{c}5 \text { SRE bundle } \\
\text { charge }\end{array}$ & 29.5 & 19.9 & 5.9 & 0.12 & 0.09 & 0.2 \\
\hline $\begin{array}{c}\text { 10 SRE bundle } \\
\text { charge }\end{array}$ & 59.0 & 39.8 & 11.9 & 0.4 & 0.17 & 0.4 \\
\hline $\begin{array}{c}4 \text { FNR + 5 } \\
\text { SRE + 19 DR- } \\
\text { 3 bundle } \\
\text { charge }\end{array}$ & 40.1 & 19.9 & 34.3 & 0.17 & 0.09 & 1.3 \\
\hline $\begin{array}{c}8 \text { SRE + 19 } \\
\text { DR-3 bundle } \\
\text { charge }\end{array}$ & 55.9 & 31.8 & 31.4 & 0.24 & 0.14 & 1.2 \\
\hline $\begin{array}{c}4 \text { FNR + 5 } \\
\text { SRE + 19 DR- } \\
\text { 3 bundle } \\
\text { charge }\end{array}$ & 46.9 & 23.3 & 40.1 & 1.5 & 0.11 & 1.5 \\
\hline $\begin{array}{c}8 \text { SRE + 19 } \\
\text { DR-3 bundle } \\
\text { charge }\end{array}$ & 65.4 & 37.2 & 36.7 & 1.4 & 0.16 & 1.4 \\
\hline
\end{tabular}

1) Dissolver volume chosen for calculating these concentrations is 14,000 L unless noted otherwise. Simulant prepared at a final $\mathrm{HNO}_{3}$ concentration of $0.5 \mathrm{M}$. Silicon concentration in this simulant is approximately $0.014 \mathrm{M}$. Mercury concentration in this simulant is approximately $0.02 \mathrm{M}$.

2) Represents a concentrated dissolver volume of $12,000 \mathrm{~L}$. Silicon concentration increases to approximately $0.016 \mathrm{M}$.

\subsection{Physical Properties}

The physical properties measured in this task were solids, density and rheology. Each of these measurements or calculations is discussed in detail below. The supernate samples were obtained by letting a subsample of the slurry settle for at least 48 hours. The free liquid (supernate) was obtained by using a pipette. The concentrated solids were also analyzed to determine the density of the undissolved solids (UDS).

\subsubsection{Solids}

The measured properties for solids analyses include the total solids in the slurry and the total solids in the supernate (or soluble solids in the supernate). These properties were determined using Equation 1 and 2:

(1) $\quad f_{\text {ts }}=\frac{m_{\text {dried slurry }}}{m_{\text {slurry }}}$

$$
f_{\text {SSS }}=\frac{m_{\text {dried supernate }}}{m_{\text {supernate }}}
$$

where:

$$
m_{\text {slurry }}=\text { mass of slurry sample (grams) }
$$


SRNL-STI-2012-00279

Revision 1

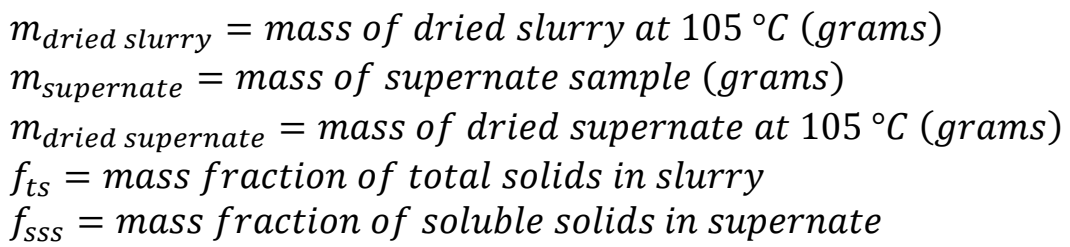

Approximately two to four grams of a sub-sample was placed into an unglazed crucible and then placed into a forced convection oven at $95{ }^{\circ} \mathrm{C}$ for a minimum of 4 hours prior to increasing the oven temperature to $105^{\circ} \mathrm{C}$ for the remainder of the analysis. The mass of the crucible and the mass of the crucible plus sub-sample were measured prior to placing it into the oven. The sample plus crucible were then measured approximately 24 and 48 hours after they were placed into the oven, where the sample was immediately taken from an oven and placed on a scale. A standard salt solution made of $20 \mathrm{wt} \% \mathrm{of} \mathrm{NaCl}$ was used to verify the operation of the oven. Triplicate samples of the slurry and supernate were measured and analyzed.

The average $\left(\bar{x}_{j}\right)$ and standard deviation $\left(\sigma_{j}\right)$ for each property was calculated using Equations 3 and 4 respectively. .

$$
\begin{aligned}
& \bar{x}_{j}=\frac{\sum_{i=1}^{n} x_{i}}{n} \\
& \sigma_{j}=\sqrt{\frac{\sum_{i=1}^{n}\left(x_{i}-\bar{x}_{j}\right)}{n(n-1)}}
\end{aligned}
$$

The fraction of undissolved solids in the slurry $\left(f_{u d s}\right)$ was calculated using Equation 5 and the STDEV $\left(\sigma_{f_{u d s}}\right)$ was calculated using Equation 6.

$$
\begin{aligned}
& f_{u d s}=\frac{f_{t s}-f_{s s s}}{1-f_{s s s}} \\
& \sigma_{f_{u d s}}=\left(\left(\frac{1}{1-f_{s s s}}\right)^{2} \sigma_{f_{t s}}^{2}+\left(\frac{f_{t s}-1}{\left(1-f_{s s s}\right)^{2}}\right)^{2} \sigma_{f_{s s s}}^{2}\right)^{\frac{1}{2}}
\end{aligned}
$$

\subsubsection{Density}

The densities of the slurry, supernate and concentrated slurry was measured using an Anton Paar DMA35 handheld density meter, see Figure $2-1$. The accuracy of the density meter is $0.001 \mathrm{~g} / \mathrm{cm}^{3}$ for solutions with viscosity $<100 \mathrm{mPa}^{*}$ s and density $<2 \mathrm{~g} / \mathrm{cm}^{3}$. The density meter was checked prior to operation by measuring the density of water at room temperature.

The DMA35 determines the density of the fluid by measuring the shift in frequency of the oscillator Utubes. The sample is pulled into the oscillator U-tubes using a vacuum plunger to fill the tubes. Air bubbles and settling solids can impact the measurement. Triplicate measurements of each slurry, supernate and concentrated slurry were made; their average and STDEVs were calculated. 
SRNL-STI-2012-00279

Revision 1

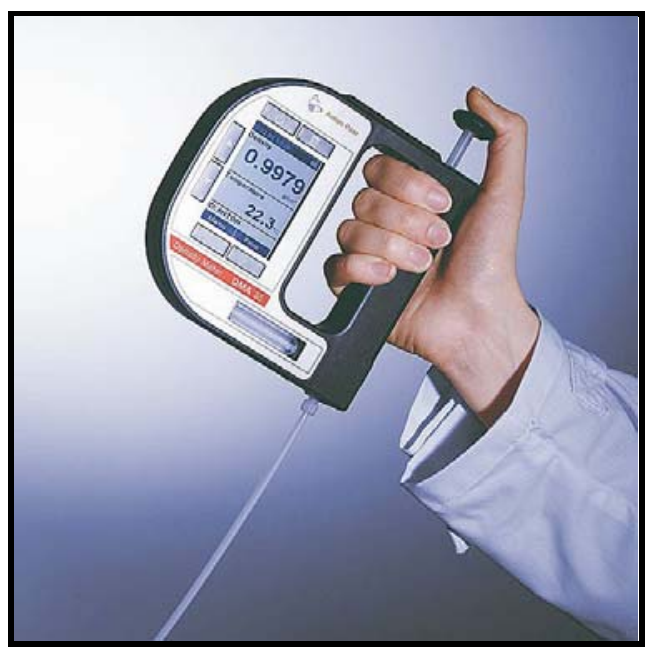

Figure 2-1. Anton Paar DMA35 Density Meter

The average and STDEV of the concentrated slurry and supernate densities and mass fraction of undissolved solids were used to determine the average density of the UDS $\left(\rho_{u d s}\right)$ and its STDEV ( $\left.\sigma_{\text {puds }}\right)$ using Equations 7 and 8 respectively.

$$
\begin{aligned}
\rho_{u d s} & =\frac{f_{u d s} \rho_{\text {sup }} \rho_{s}}{\rho_{\text {sup }}+\rho_{s}\left(f_{u d s}-1\right)} \\
\sigma_{\rho_{u d s}} & =\frac{\left[\left(\rho_{\text {sup }}^{2} \rho_{s}-\rho_{s}^{2} \rho_{\text {sup }}\right)^{2} \sigma_{f_{u d s}}^{2}+\left(\rho_{s}^{2} f_{u d s}\left(f_{u d s}-1\right)\right)^{2} \sigma_{\rho_{s u p}}^{2}+\left(\rho_{s}^{2} f_{u d s}\right)^{2} \sigma_{\rho_{S}}^{2}\right]^{\frac{1}{2}}}{\left(\rho_{\text {sup }}+\rho_{s}\left(f_{u d s}-1\right)\right)^{2}}
\end{aligned}
$$

\subsubsection{Particle Size Distribution}

The particle size distribution (PSD) was determined using the Microtrac X100. The Microtrac X-100 particle size analyzer uses a wet sample delivery controller (recirculator) to disperse the sample uniformly in a fluid and deliver the sample to the analyzer. This wet sample delivery controller in its basic form consists of a reservoir where the sample is introduced, a fluid pump, a valve to the drain system, and the necessary tubing connections to the analyzer. The flow through the analyzer sample cell is always from the bottom to the top. A laser beam is projected through a transparent quartz cell containing a stream of moving particles suspended in deionized water. Light from the laser strikes the particles and is scattered through various angles. The scattering angles and intensities of the scattered light are measured by two photodiode arrays producing electronic signals proportional to the measured light flux. The Microtrac proprietary mathematical software processes the signals to obtain a particle size distribution. Upon completion of the analysis, the Microtrac generates a report containing the tabular data, a histogram plot of the data, and various instrument parameters.

The volumetric particle size distribution is used. The mean volume and 90 percentile particle sizes are used to support the various calculations below. It is assumed that the densities of the various precipitated particles are the same when using the PSD data for calculations, though in reality this is mostly not the case, but it is impractical (or impossible) to measure the individual flocculated materials or compounds. If the measured PSDs are similar, the data sets were averaged to determine the percentile data, mean volume, mean area, and mean number sizes and reported. 
The slurry samples were diluted using simulant salt solutions that were determined by reviewing the ICP-ES data of the various supernatants from the 14,000 L slurry batches. The salt simulants are required to provide the necessary dilution for the PSD measurement and to minimize any effect of dissolution with the undissolved solids in the actual wastes. After the salt solutions were made, they were processed through a 0.25 micron absolute filter to remove any undissolved solids greater than the filter size. The salt solutions used for the 0.8 and 1.2 M slurries are provided in Table 2-3.

Table 2-3 Salt Simulants Used for PSD Measurements

\begin{tabular}{|c|c|c|}
\hline Compound/Property & 1.2 M Simulant & 0.8 M Simulant \\
\hline DI water $(\mathrm{g})$ & 842.06 & 774.04 \\
\hline Aluminum Nitrate $+9 \mathrm{H}_{2} \mathrm{O}(\mathrm{g})$ & 90.92 & 106.43 \\
\hline Sodium Nitrate $(\mathrm{g})$ & 34.60 & 205.87 \\
\hline $50 \mathrm{wt} \%$ Sodium Hydroxide $(\mathrm{g})$ & 140.72 & 114.07 \\
\hline Density $(\mathrm{g} / \mathrm{mL})$ & 1.1083 & 1.2004 \\
\hline
\end{tabular}

The particle size distributions of the materials processed at SRNL are expected to be larger than those of the fluids processed in $221-\mathrm{H}$. The neutralization process at the $221-\mathrm{H}$ occurs in an agitated vessel, where the impellers are flat blades ${ }^{4,5,6}$ and the addition rate of caustic solution occurs over a period of hours ${ }^{7}$. The flat blade design can provide a high level of shearing and dispersion of the caustic fluid during the neutralization process which was not utilized during the SRNL feed prep. Additional the slow rate of caustic addition can reduce the resulting flocculated particle size distribution. ${ }^{7}$ Extensive work in simulant sludge preparation for DWPF simulants have shown that there are multiple variables that can impact the PSD during the neutralization process, such as mixer speed, changes in $\mathrm{pH}$, caustic addition rate, and scaling. ${ }^{8,9,10}$ In these studies, the effect of particles less than 1 micron in diameter seem to have the most drastic impact on rheology.

\subsubsection{Rheology}

The rheological properties of the dissolver simulant fluids were measured using a Haake VT550 rotoviscometer. Visual inspection of the samples showed they contained solids and the solution was viscous in nature, indicating that the MV1 bob/cup configuration would be utilized to perform the measurements. The MV1 bob/cup configuration is a concentric cylinder, where the inner cylinder rotates and the outer cylinder is fixed. The VT550 controls the rotational speed and measures both the rotational speed and measured torque on the rotating cylinder. Given the geometry of the MV1/bob/cup, the measured shear stress and shear rates are calculated by the Haake software. The shear rates are those for Newtonian fluids and if the fluid does have non-Newtonian behavior, corrections to the shear rate are not be performed. Corrections to the shear rate for non-Newtonian behavior will increase the shear rate for any given point, resulting in the decrease in the plastic viscosity, given that the fluid is shear thinning, which is the case for fluids processed at SRS. Flow curve measurements utilized an existing tank farm flow curve profile listed in Table 2-4. A National Institute of Standards and Testing (NIST) traceable Newtonian viscosity standard was used to verify the operability of the VT550 roto-viscometer on a daily basis of use, where the calculated viscosity to the flow curve is within $\pm 10 \%$ of the NIST oil standard viscosity at $25{ }^{\circ} \mathrm{C}$. Measurements of the fluids in this task were performed at $20{ }^{\circ} \mathrm{C}$ in duplicate. Samples were prepared by vigorously shaking the bottle to breakup flocculated material, swirling to assist in removing entrained air, loading into the cup, raising into to heating/cooling bath, trimming excess fluid and starting the measurement using the sludge flow curve profile. 
Table 2-4. Sludge Flow Curve Profile Using MV1 Geometry

\begin{tabular}{|c|c|c|}
\hline \multicolumn{3}{|c|}{ Shear rate and time of measurement } \\
\hline Up Curve & Hold & Down Curve \\
\hline $\begin{array}{c}0 \text { to } 600 \mathrm{~s}^{-1} \text { linearly } \\
\text { in } 5 \mathrm{~min}\end{array}$ & $600 \mathrm{~s}^{-1}$ for $1 \mathrm{~min}$ & $\begin{array}{c}600 \text { to } 0 \mathrm{~s}^{-1} \text { linearly } \\
\text { in } 5 \mathrm{~min}\end{array}$ \\
\hline
\end{tabular}

The resulting up and down flow curves were linearly regressed using the following rheological models:

(9) Newtonian: $\tau=\mu \dot{\gamma}$

(10) Bingham Plastic: $\tau=\tau_{o}+\eta_{\infty} \dot{\gamma}$

where:

$$
\begin{aligned}
& \tau=\text { measured shear stress }(\mathrm{Pa}) \\
& \mu=\text { viscosity }(\mathrm{Pa} \cdot \mathrm{s}) \\
& \tau_{o}=\text { Bingham Plastic Yield Stres }(\mathrm{Pa}) \\
& \eta_{\infty}=\text { plastic viscosity }(\mathrm{Pa} \cdot \mathrm{s}) \\
& \dot{\gamma}=\text { shear rate }(1 / \mathrm{s})
\end{aligned}
$$

The average apparent viscosity at the maximum shear rate was defined as:

(11) Apparent Viscosity at maximum shear rate: $\eta_{600 \mathrm{~s}^{-1}}=\left(\frac{\tau}{\dot{\gamma}}\right)_{600 \mathrm{~s}^{-1}}$

\subsubsection{Method to Determine Average Velocity and Volumetric Flow Rate}

The flow rate in the gravity waste transfer lines was determined using the energy Equation $12 .{ }^{11}$ This energy Equation assumes the flow is turbulent and this assumption will be verified.

$$
\frac{\mathrm{P}_{1}}{g \rho_{1}}+\mathrm{z}_{1}+\frac{\mathrm{V}_{1}^{2}}{2 \mathrm{~g}}=\frac{\mathrm{P}_{2}}{\mathrm{~g} \rho_{2}}+\mathrm{z}_{2}+\frac{\mathrm{V}_{2}^{2}}{2 \mathrm{~g}}+\mathrm{h}_{\mathrm{L}}
$$

where:

$$
\begin{aligned}
& P_{i}=\text { pressure }\left(\frac{l b f}{f t^{2}}\right) \\
& \rho_{i}=\text { fluid density }\left(\frac{l b m}{f t^{3}}\right) \\
& z_{i}=\text { elevation above a datum }(f t) \\
& V_{i}=\text { average fluid velocity }\left(\frac{f t}{s}\right) \\
& g=\text { gavitational acceleration }\left(\frac{f t}{s^{2}}\right) \\
& h_{L}=\text { head losses }(f t) \\
& i=\text { any two points in the hydraulic system }
\end{aligned}
$$

The average fluid velocity and hydraulic radius are given as Equation 13 and 14 respectively.

$$
\mathrm{V}=\frac{\dot{\mathrm{Q}}}{\mathrm{A}}
$$




$$
R=\frac{A}{P_{w}}
$$

where:

$$
\begin{aligned}
& R=\text { hydraulic radius }(f t) \\
& A=\text { cross }- \text { sectional area of flow }\left(f t^{2}\right) \\
& P_{w}=\text { wetted perimeter }(f t) \\
& \dot{Q}=\text { volumemetric flow rate }\left(\frac{f t^{3}}{s}\right)
\end{aligned}
$$

The head losses associated with the waste lines include entrance, piping, elbows, and exit losses. These losses are determined using the following Equations $15,16,17$ and $18^{11,12}$ :

$$
\begin{aligned}
& \text { Entrance: } \quad h_{\text {entrance }}=K_{\text {entrance }} \frac{V^{2}}{2 g}=0.5 \frac{V^{2}}{2 g} \\
& \text { Exit: } \quad h_{\text {exit }}=K_{\text {exit }} \frac{V^{2}}{2 g}=1 \frac{V^{2}}{2 g} \\
& \text { Piping: } h_{\text {piping }}=f_{T} \frac{L}{R} \frac{V^{2}}{8 g} \\
& \text { Elbow: } h_{\text {elbow }}=K_{\text {elbow }} \frac{V^{2}}{2 g}=f_{T} K_{\frac{r}{d}} \frac{V^{2}}{2 g}
\end{aligned}
$$

where:

$$
\begin{aligned}
& f_{T}=\text { turbulent friction factor (unitless) } \\
& L=\text { length of piping }(f t) \\
& K_{\bar{d}}=\text { constant for specific pipe }\left(\frac{r}{d}\right) \text { bend in turbulent flow }(\text { see page } A-29)^{12}
\end{aligned}
$$

The total frictional loss is the sum of the above losses and the results are shown in Equation 19.

$$
h_{L}=0.5 \frac{V^{2}}{2 g}+\frac{V^{2}}{2 g}+\sum_{i=1}^{n} f_{T} K_{\frac{r}{d}}, \frac{V^{2}}{2 g}+f_{T} \frac{L}{R} \frac{V^{2}}{8 g}=1.5 \frac{V^{2}}{2 g}+\sum_{i=1}^{n} f_{T} K_{\frac{r}{d}}, i \frac{V^{2}}{2 g}+f_{T} \frac{L}{4 R} \frac{V^{2}}{2 g}
$$

In the case of gravity flow, $\mathrm{P}_{1} \cong \mathrm{P}_{2}$ since the pressure where the waste enters the header in Building $221-\mathrm{H}$ and exiting into the H Pump Pit \#5 or \#6 are essentially the same. It is also assumed that the pipe is partially full and the inlet and outlet velocities are the same. The differences in inlet/outlet velocities would have little impact in the overall hydraulics of the system, due to the length of piping and other minor frictional losses. Additionally, the effect of air entrainment on the hydraulics of the system or filling of the pipe was ignored. The effect of air entrainment can drastically impact the hydraulics, reducing the flowrate. ${ }^{13,14,15}$ Equation 12 reduces to Equation 20.

$$
z_{1}-z_{2}=h_{L}=1.5 \frac{V^{2}}{2 g}+\sum_{i=1}^{n} f_{T} K \frac{r}{d^{\prime}}, \frac{V^{2}}{2 g}+f_{T} \frac{L}{4 R} \frac{V^{2}}{2 g}
$$

The loss coefficients used above are for turbulent flow conditions and can be determined using Reynolds number $\left(\mathrm{N}_{\mathrm{RE}}\right)$, Equation 21, and the flow is considered turbulent when $\mathrm{N}_{\mathrm{RE}}$ is greater than 4,000 .

$$
N_{R E}=\frac{4 V \rho R}{\mu}
$$

where:

$$
\rho=\text { density of the fluid }\left(\frac{l b m}{f t^{3}}\right)
$$




$$
\mu=\text { fluid viscosity }\left(\frac{l b m}{f t \cdot s}\right)
$$

The Darcy-Weisbach friction factor for fully developed turbulent flow in conduit, $\mathrm{f}_{\mathrm{T}}$, can be determined using the Colebrook-White Equation for filled conduit, Equation 22. ${ }^{11}$ This Equation was solved iteratively when coupled with Equation 20.

$$
\begin{aligned}
& \frac{1}{\sqrt{f_{T}}}=-2 \log \left(\frac{k}{14.8 R}+\frac{2.51}{N_{R E} \sqrt{f_{T}}}\right) \\
& \frac{1}{\sqrt{f_{T}}}=-2 \log \left(\frac{k}{12 R}+\frac{2.51}{N_{R E} \sqrt{f_{T}}}\right)
\end{aligned}
$$

where:

$$
k=\text { pipe roughness }(f t)
$$

For a Reynolds number between the 2100 and 4000, the flow is considered transitional, where the friction factor cannot be explicitly determined as shown in the Moody diagram (Figure 2-2) for Newtonian fluids ${ }^{16}$. If the calculated Reynolds number is in the transitional or "critical" regime, the turbulent friction factor will be used since it will yield a lower flow rate.

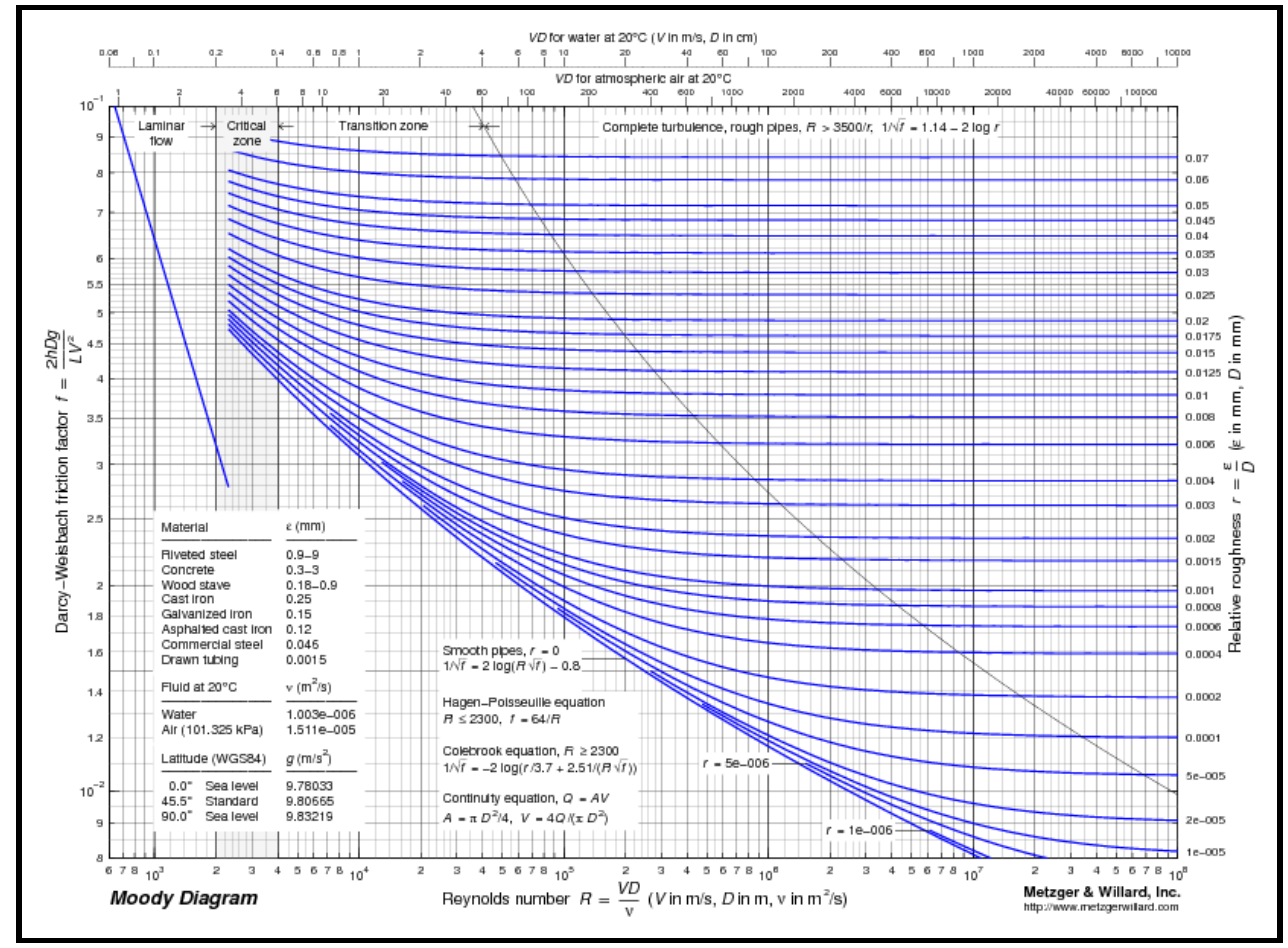

Figure 2-2. Moody Diagram

\subsubsection{Methods to Determine Non-Newtonian pipe frictional losses}

The slurries will be analyzed as a Bingham Plastic fluid. Two dimensionless parameters are required to further assess the pressure drop associated with this model and they are the Reynolds Bingham Number (Equation 24) and the Hedstrom number (Equation 25). 


$$
\begin{aligned}
& R e_{B}=\frac{4 R V \rho}{\eta_{\infty}} \\
& H e=\frac{16 R^{2} \rho \tau_{o}}{\eta_{\infty}^{2}}
\end{aligned}
$$

For non-Newtonian flow, the pressure drop can be determined using the following relationship developed for a Bingham Plastic fluid. ${ }^{17}$ Note that pipe roughness is not included in the turbulent term; hence, this calculated value could be lower than expected, but no correction will be performed. The laminar and turbulent friction factors are determined using Equations 26 and 27:

$$
\begin{aligned}
& \left.f_{B P-L} \approx \frac{16}{R e_{B}}\left[1+\frac{H e}{6 R e}\right] \text { (Laminar }\right) \\
& f_{B P-T}=10^{a} R e_{B}^{b}(\text { Turbulent })
\end{aligned}
$$

where:

$$
\begin{aligned}
& f_{B P-L}=\text { Bingham Plastic laminar friction factor } \\
& f_{B P-T}=\text { Bingham Plastic Turbulent friction factor } \\
& \begin{array}{l}
a=-1.47\left[1+0.146 e^{-2.9 \times 10^{-5} \cdot H e}\right] \\
b=-0.193
\end{array}
\end{aligned}
$$

The laminar and turbulent friction factor can be combined a single friction factor, Equation 28 . Note the factor of 4. This is required to convert the Fanning friction factor to the Darcy friction factor which was used to determine the pressure drop.

$$
f_{B P}=4 \cdot\left(f_{B P-T}^{m}+f_{B P-L}^{m}\right)^{\left(\frac{1}{m}\right)}
$$

where: $m=1.7+\frac{40,000}{R e_{B}}$

Correcting the minor losses if the flow becomes laminar will not be performed. By not performing such a calculation, the calculated velocity will be larger since the loss coefficient is larger in laminar flow as compared to turbulent flow. Laminar minor loses can be determined using either the $2-\mathrm{K}$ or $3-\mathrm{K}$ method, though losses for very long radius elbows as those in these drain lines are not provided. ${ }^{18}$

\subsubsection{Impact of Partial Pipe Fill on Solids Suspension}

The previous calculations were based on the assumption that the pipe is full of fluid when determining the hydraulic losses for either a Newtonian or Bingham Plastic fluid. In this section, the velocity in various sections of horizontal piping is investigated to determine if the velocity is adequate to maintain the undissolved solids in suspension. The procedure consists of determining the height of fluid in the various sloped sections of piping and then using a simple correlation.

Assuming that the inlet/outlet and minor losses are ignored in sloped sections of piping and the fluid level is the same for a given pipe slope, Equation 19 reduces to Equation 29:

$$
z_{1}-z_{2}=f_{T} \frac{L}{4 R} \frac{V^{2}}{2 g}(f t)
$$

Or solving for the slope of the pipe (S) we obtain Equation 30: 


$$
S=\frac{z_{1}-z_{2}}{L}=\frac{f T}{4 R} \frac{V^{2}}{2 g}(f t / f t)
$$

The hydraulic radius for a partially filled (or full) pipe can be determined using Figure 2-3.

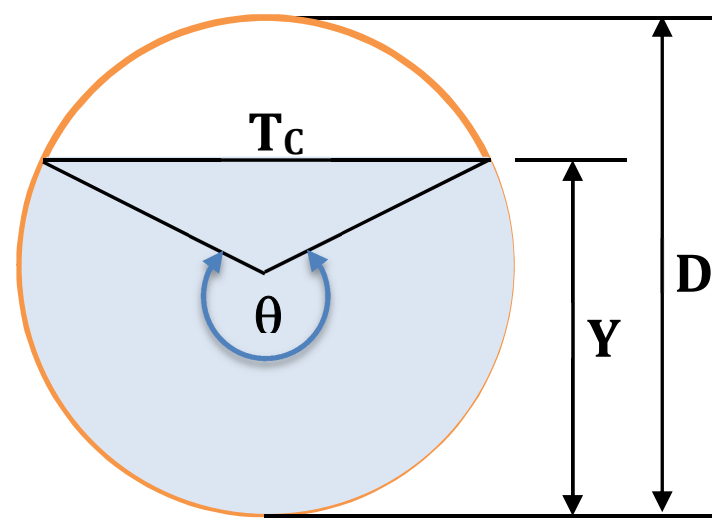

Figure 2-3. Partial Pipe Fill to Determine the Hydraulic Radius for a Given Fill Height

where:

$$
\begin{aligned}
& \mathrm{D}=\text { diameter of pipe }(\mathrm{ft}) \\
& \mathrm{Y}=\text { Fill Height }(\mathrm{ft}) \\
& F=\text { Fill Factor }=\frac{Y}{D} \text { (dimensionless) } \\
& \theta=2 \cdot \operatorname{acos}(1-2 \cdot F) \text { (radians) } \\
& T_{C}=\text { cord length at fill height }=D \sin \left(\frac{\theta}{2}\right)(\mathrm{ft}) \\
& A=\text { cross sectional area of flow }=\frac{D^{2} \cdot(\theta-\sin \theta)}{8}\left(\mathrm{ft}^{2}\right) \\
& P_{w}=\text { wetted perimeter }=\frac{D \cdot \theta}{2}(\mathrm{ft})
\end{aligned}
$$

The definition of the hydraulic radius is given by Equation 14 and substituting in the variables above yields Equation 31 which is the hydraulic radius for any pipe fill:

$$
R=\frac{\frac{D^{2} \cdot(\theta-\sin \theta)}{\frac{D}{2}}}{\frac{D \cdot \theta}{2}}=\frac{D \cdot(\theta-\sin \theta)}{4 \cdot \theta}
$$

The hydraulic radius is used in the energy Equation, Reynolds number, and friction coefficient. The friction factor for partially filled pipe for Newtonian fluids can be determined using Equation 23 and the non-Newtonian as previously described.

The energy Equation 30 in this case is solved using the goal seek option in EXCEL. The Newtonian frictional factor (Equation 23) is determined using eight iterations and the non-Newtonian friction factor is determined using Equation 28. Inputs for these calculations are the actual flowrate provided by the jet transfer pump, fluid properties, clean pipe roughness, and the pipe inside diameter. 


\subsubsection{Determining Deposition Velocity and Sediment Transport}

The deposition velocity (i.e., velocity at which particles will settle out of the flow stream) will be determined assuming the particles are considered hard bodies (e.g., they are not considered flocculated material that contains interstitial fluids) and the particles are not cohesive. The assumption that these freshly made solids are hard bodies makes this calculation conservative, since the interstitial fluids would reduce the "average" density of the particle as determined using the light scattering results.

In gravity flow, the flow can be subcritical, critical or supercritical, based on the Froude number. At critical flow, $\mathrm{Fr}=0$, the specific energy is at a minimum and the velocity head is half the hydraulic depth of the channel for small slopes, but this condition is very unstable. When the channel slope is less than that for critical flow, the flow is considered subcritical $(\mathrm{Fr}<1)$, resulting in a slower flow. When the channel slope is steeper than the critical slope, then the flow is faster.

The deposition velocity for open channel flow is shown in Equation 32. ${ }^{16}$ The form of this equation is consistent with that used for completely filled piping used to transport non-cohesive solids, though the power coefficients maybe slightly different and other physical properties considered. ${ }^{19}$

$$
V_{D}=1.833\left[\frac{8 g R}{\rho}\left(\rho_{S}-\rho\right)\right]^{\frac{1}{2}}\left(\frac{d_{85}}{R}\right)^{0.158}\left(\frac{f t}{s e c}\right)
$$

where:

$$
\begin{aligned}
& V_{D}=\text { deposition velocity } \\
& \rho_{S}=\text { density of solid } \\
& d_{85}=\text { particle size of the } 85 \% \text { by volume }
\end{aligned}
$$

Sediment transport is another model that can be used to determine how to treat the particulate material once it is in suspension and the mass flux of the particles in the flow. One of the methods is the Ackers and White model, which is based on a physical model for open channel transport process. ${ }^{20,21}$ In this model, a dimensionless grain diameter $\mathrm{d}_{\mathrm{gr}}$ is calculated (Equation 33) and if this value is between 1 and 60 the sediment is transported in suspension (as part of the fluid) and if it is greater than 60 the particles are considered coarse. If $\mathrm{d}_{\mathrm{gr}}$ is less than 1 , than this method does not apply since the solids tend to be cohesive and no calculation will be performed.

$$
\begin{aligned}
& d_{g r}=d_{s}\left[\frac{g\left(\frac{\rho_{S}}{\rho}-1\right)}{v^{2}}\right]^{\frac{1}{3}} \quad(\text { dimensionless }) \\
& n^{\prime}=1.00-0.56 \cdot \log \left(d_{g r}\right)(\text { dimensionless }) \\
& A^{\prime}=\frac{0.23}{d_{g r}^{0.5}}+0.14 \text { (dimensionless) } \\
& m^{\prime}=\frac{6.83}{d_{g r}}+1.67 \quad(\text { dimensionless }) \\
& C=10^{a \prime}(\mathrm{N})(\text { dimensionless }) \\
& a^{\prime}=2.79 \log \left(d_{g r}\right)-\left(\log \left(d_{g r}\right)\right)^{2}-3.46 \quad \text { (dimensionless) }
\end{aligned}
$$




$$
\begin{aligned}
& u_{*}=\left(g d_{h} S\right)^{0.5}\left(\frac{f t}{\mathrm{sec}}\right) \\
& F=\frac{u_{*}^{n^{\prime}}}{\left[g d_{s}\left(\frac{\rho_{S}}{\rho}-1\right)\right]^{0.5}} \cdot\left[\frac{V}{5.66 \cdot \log \left(\frac{10 d_{h}}{d_{s}}\right)}\right]^{1-n} \quad \text { (dimensionless) } \\
& G=C\left(\frac{F}{A^{\prime}}-1\right)^{m^{\prime}}(\text { dimensionless) } \\
& X=G \cdot \frac{\rho_{S}}{\rho} \cdot \frac{d_{s}}{d_{h}} \cdot\left(\frac{V}{u_{*}}\right)^{n^{\prime}}\left(\frac{l b m}{l b m}\right)
\end{aligned}
$$

where:

$$
\begin{aligned}
& d_{g r}=\text { dimensionless grain diameter } \\
& n^{\prime}, A^{\prime}, m^{\prime}, C, a^{\prime}=\text { variou parameters } \\
& u_{*}=\text { shear or friction velocity } \\
& F=\text { Particle Mobility } \\
& G=\text { dimensionless sediment transport function } \\
& X=\text { mass of sediment transported per unit mass of fluid flow }
\end{aligned}
$$

Review of the piping drawings ${ }^{22,23,24,25,26,27,28}$ for the waste transfer line (or waste headers) between Building 221-H and HPP\#5 or HPP\#6 are summarized in Table 2-5 for the data necessary to evaluate Equation 12. The waste lines running parallel to the east wall of Building 221-H consists of 10-inch headers that reduce to 3 -inch schedule 40 pipe in at the transition box next to Section 4 of the building and finally discharging into HPP\#5 or HPP\#6. Lines WF1100 (WH\#1, HL-1), WF1101 (WH\#4, LL-4), and WF1102 (WH\#3, LL-3) have vertical drops that occur at the transition box Building 221-H. There is no vertical drop in the 3-inch line after leaving the 10-inch header for WF1103 (WH\#2, HL-2), hence this flow circuit has the lowest driving head. The slopes (vertical/horizontal distances) in the all the transfer lines has a minimum of 0.005 and a maximum of 0.29 . The minimum slope was specified in reference 29 . 
Table 2-5. Complete Elevation, Piping Run, Elbows, Entrance and Exit Data for Waste Transfer Line between Building 221-H to HPP\#5 and HPP\#6

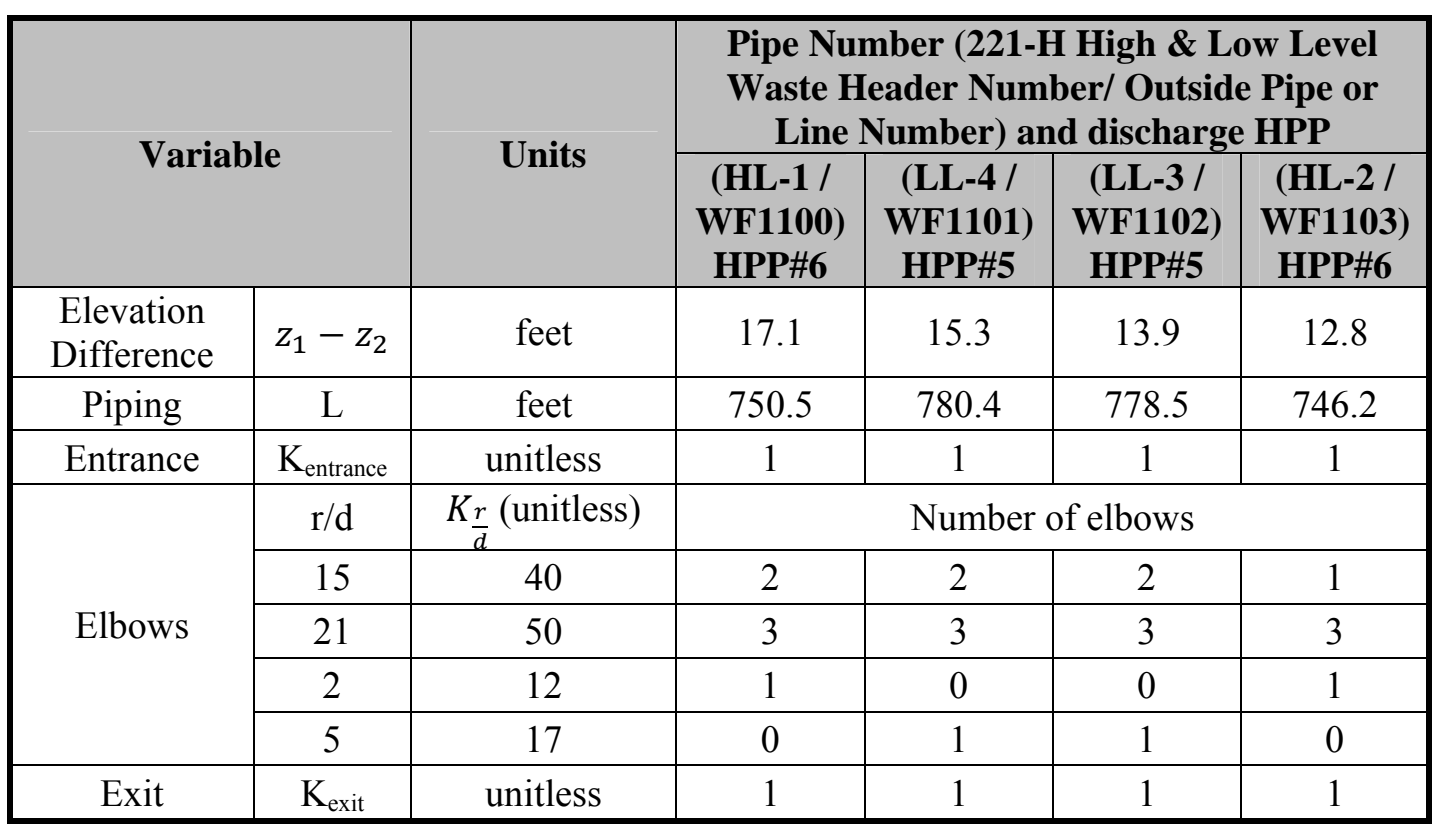

All the transfer lines leaving Building 221-H initially have a slope of 0.005 and a calculation was performed to determine the velocity that this section of piping can handle. Table 2-6 provides the various lengths of piping and fittings associated with this initial section of the waste header.

\section{Table 2-6. Initial Elevation Drop and 0.005 Sloped Piping Run, Elbows, Entrance for Waste Transfer Exiting Building 221-H}

\begin{tabular}{|c|c|c|c|c|c|c|}
\hline \multirow{2}{*}{} & \multirow{2}{*}{ Variable } & \multicolumn{3}{c|}{$\begin{array}{c}\text { Pipe Number (221-H Waste Header/ Outside } \\
\text { Line Number) and discharge HPP }\end{array}$} \\
\cline { 4 - 8 } & Units & $\begin{array}{c}\text { (HL-1/ } \\
\text { WF1100) } \\
\text { HPP\#6 }\end{array}$ & $\begin{array}{c}\text { (LL-4 / } \\
\text { WF1101) } \\
\text { HPP\#5 }\end{array}$ & $\begin{array}{c}\text { (LL-3 / } \\
\text { WF1102) } \\
\text { HPP\#5 }\end{array}$ & $\begin{array}{c}\text { WL-2 / } \\
\text { WF1103) } \\
\text { HPP\#6 }\end{array}$ \\
\hline $\begin{array}{c}\text { Elevation } \\
\text { Difference }\end{array}$ & $z_{1}-z_{2}$ & feet & 4.91 & 3.12 & 1.71 & 0.64 \\
\hline Piping & $\mathrm{L}$ & feet & 115.5 & 112.2 & 112.3 & 111.2 \\
\hline Entrance & $\mathrm{K}_{\text {entrance }}$ & unitless & 1 & 1 & 1 & 1 \\
\hline \multirow{3}{*}{ Elbows } & $\mathrm{r} / \mathrm{d}$ & $K_{\frac{r}{d}}$ (unitless) & \multicolumn{5}{|c|}{ Number of elbows } \\
\cline { 2 - 8 } & 15 & 40 & 2 & 2 & 2 & 1 \\
\cline { 2 - 8 } & 21 & 50 & 1 & 1 & 1 & 1 \\
\hline
\end{tabular}

The waste generated in Building $221-\mathrm{H}$ is transferred to the 10 -inch header using an S-2 steam jet ${ }^{30}$, which can supply flow up to 30 gallon per minute $(\mathrm{gpm}){ }^{30}$ The nominal flow rate provided by the S-2 steam jet is typically around $25 \mathrm{gpm}$.

Calculations for both the complete hydraulic system and that of the initial sloped section were calculated. The pipe roughness used in the calculations for a clean pipe $(k=0.00015 \mathrm{ft})$ is about 44 times smaller than for a rusted pipe $(k=0.00667 \mathrm{ft}){ }^{31}$ The pipe roughness for a rusted pipe was selected based on discussion with R. Eubanks on the operations of this header, where flushing after each transfer is not 
normally performed. The actual roughness is not known for these transfer lines, but this example shows the impact that potential material buildup can have on flow.

Water and a limiting dissolver fluid (highest viscosity / lowest density) were used in the calculations to determine the velocity, Reynolds number, and flow rate. These water calculations provide an upper bound to flow rates. For the 12,000 L batched slurries that have significant non-Newtonian properties, their rheological data as well as their Newtonian fit to the flow curve will be used to determine this parameters.

Table 2-7 is a summary of the various sections of sloped piping leaving Building 221-H that are greater than 40 feet in length and their respective slopes. Slopes of 0.005 and $0.0268 \mathrm{ft} / \mathrm{ft}$ will be used in calculations to determine the velocity, fill ratio, and Reynolds number for all the characterized fluids.

Table 2-7 Sloped Piping Run

\begin{tabular}{|c|c|c|c|c|c|c|}
\hline \multicolumn{3}{|c|}{ Sloped Piping Run } & $\begin{array}{c}\text { (HL-1 / } \\
\text { WF1100) } \\
\text { HPP\#6 }\end{array}$ & $\begin{array}{c}\text { (LL-4 / } \\
\text { WF1101) } \\
\text { HPP\#5 }\end{array}$ & $\begin{array}{c}(\text { LL-3 / } \\
\text { WF1102) } \\
\text { HPP\#5 }\end{array}$ & $\begin{array}{c}\text { (HL-2 / } \\
\text { WF1103) } \\
\text { HPP\#6 }\end{array}$ \\
\hline \multirow{3}{*}{1} & Length & $\mathrm{ft}$ & 43.77 & 42.27 & 42.27 & 42.27 \\
\cline { 2 - 7 } & Slope & $\mathrm{ft} / \mathrm{ft}$ & 0.0050 & 0.0054 & 0.0054 & 0.0054 \\
\hline \multirow{2}{*}{2} & Length & $\mathrm{ft}$ & 58.25 & 58.25 & 59.75 & 59.75 \\
\cline { 2 - 7 } & Slope & $\mathrm{ft} / \mathrm{ft}$ & 0.0050 & 0.0050 & 0.0050 & 0.0050 \\
\hline \multirow{3}{*}{3} & Length & $\mathrm{ft}$ & 206 & 206 & 206 & 206 \\
\cline { 2 - 7 } & Slope & $\mathrm{ft} / \mathrm{ft}$ & 0.0268 & 0.0268 & 0.0268 & 0.0268 \\
\hline \multirow{3}{*}{4} & Length & $\mathrm{ft}$ & 177.5 & 177.5 & 179 & 179 \\
\cline { 2 - 7 } & Slope & $\mathrm{ft} / \mathrm{ft}$ & 0.0134 & 0.0134 & 0.0131 & 0.0131 \\
\hline \multirow{2}{*}{5} & Length & $\mathrm{ft}$ & 91.42 & 91.42 & 92.92 & 92.92 \\
\cline { 2 - 7 } & Slope & $\mathrm{ft} / \mathrm{ft}$ & 0.0135 & 0.0135 & 0.0131 & 0.0131 \\
\hline \multirow{2}{*}{6} & Length & $\mathrm{ft}$ & 113.08 & 113.08 & 111.08 & 111.08 \\
\cline { 2 - 7 } & Slope & $\mathrm{ft} / \mathrm{ft}$ & 0.0150 & 0.0150 & 0.0050 & 0.0050 \\
\hline \multirow{2}{*}{7} & Length & $\mathrm{ft}$ & 47 & 47 & 46 & 46 \\
\cline { 2 - 7 } & Slope & $\mathrm{ft} / \mathrm{ft}$ & 0.0155 & 0.0155 & 0.0050 & 0.0050 \\
\hline
\end{tabular}

\subsection{Results and Discussion}

\subsection{Surface Area Calculations $^{1}$}

Before any off-gas generation calculations can be performed for the dissolution of various UNF assemblies in the H-Canyon dissolvers, surface area calculations per unit height need to be performed for the L-Bundle into which the various UNF assemblies are placed. Table 3-1 shows the total surface area (inside and outside) values for the L-Bundle along with the SRE, HMI ${ }^{32}$, and DR-3 assemblies. ${ }^{2}$ The yellow row in Table 3-1 represents a 54 inch $(4.5 \mathrm{ft})$ immersion into the dissolver and the pink row represents the approximate maximum height $(11 \mathrm{ft})$ inside the L-Bundle for placing the UNF assemblies. For comparison, the MURR and L-Bundle Total Surface Area (inside and outside) values from a previous

\footnotetext{
${ }^{1}$ HMI was originally selected for evaluation but was subsequently removed from planned dissolving recipes.
} 
calculation $^{33}$ are shown in Table 3-1. Table 3-1 plots the total surface area data from and shows that the MURR assembly from prior dissolver campaigns has a much higher surface area per unit height value than the SRE, HMI, and DR-3 assemblies.

In a prior report for the dissolution of the MURR assemblies ${ }^{34}$ it was shown that the total surface areas (inside and outside) of the L-Bundle and the MURR assemblies should not be used in off-gas generation calculations as actual plant data shows that the vacuum system was not overwhelmed by the dissolving of the MURR assemblies. As stated in the prior report this restriction of only using the outer surface areas is due to mass transfer limitations of nitric acid and mercury to the metal surfaces, heat transfer from the surfaces due to heat of dissolution, and the large amount of gas generated and associated bubbles at the surfaces. Since the external shape and size of the MURR assembly is comparable to the external shape and size of the SRE, HMI, and DR-3 assemblies, the same assumption for using only the outer surfaces also applies. In addition, the prior report for the MURR assemblies assumed that the peak off-gas rates for the L-Bundle and MURR assemblies occur at different times. In other words, the outer surface area of the L-Bundle is the primary contributor to the off-gas generation until it dissolves exposing the outer surface area of the assembly, which then becomes the primary off-gas contributor. Following these assumptions, only the outer surface areas of the L-Bundle and the appropriate surface areas of the new UNF assemblies (SRE, HMI, and DR-3) that were used in the off-gas calculations are shown in Table 3-2.

The details of the surface area calculations for the Disassembly L-Area Bundling Tube along with the SRE, HMI, and DR-3 (Denmark Reactor) assemblies are shown in Appendix A.

Table 3-1. L-Bundle and UNF Assemblies Total Surface Areas per Immersion Height

\begin{tabular}{|c|c|c|c|c|c|c|c|}
\hline $\begin{array}{c}\text { Assembly } \\
\text { Immersion } \\
\text { Depth [ft] }\end{array}$ & $\begin{array}{c}\text { MURR \& } \\
\text { L-Bundle } \\
\text { Total } \\
\text { Surface } \\
\text { Area }\left[\mathrm{ft}^{2}\right] \\
\end{array}$ & \begin{tabular}{|c|} 
Outer SRE \\
\& L-Bundle \\
Total \\
Surface Area \\
{$\left[\mathrm{ft}^{2}\right]$} \\
\end{tabular} & \begin{tabular}{|c|} 
Outer \& \\
Inner SRE \& \\
L-Bundle \\
Total Surface \\
Area $\left[\mathrm{ft}^{2}\right]$ \\
\end{tabular} & \begin{tabular}{|c|} 
HMI-Std \& \\
L-Bundle \\
Total \\
Surface \\
Area $\left[\mathrm{ft}^{2}{ }^{2}\right]$ \\
\end{tabular} & $\begin{array}{c}\text { HMI-Ctrl \& } \\
\text { L-Bundle } \\
\text { Total } \\
\text { Surface } \\
\text { Area }\left[\mathrm{ft}^{2}\right] \\
\end{array}$ & $\begin{array}{c}\text { DR-3-1 \& } \\
\text { L-Bundle } \\
\text { Total } \\
\text { Surface } \\
\text { Area }\left[\mathrm{ft}^{2}\right] \\
\end{array}$ & $\begin{array}{c}\text { DR-3-2 \& } \\
\text { L-Bundle } \\
\text { Total } \\
\text { Surface } \\
\text { Area }\left[\mathrm{ft}^{2}\right] \\
\end{array}$ \\
\hline 0.000 & 0.77 & 0.65 & 0.65 & 0.65 & 0.65 & 0.65 & 0.65 \\
\hline 0.083 & 1.74 & 1.13 & 1.15 & 1.16 & 1.16 & 1.23 & 1.23 \\
\hline 0.167 & 3.16 & 1.42 & 1.45 & 1.47 & 1.54 & 1.58 & 1.58 \\
\hline 0.250 & 4.57 & 1.71 & 1.75 & 1.77 & 1.84 & 1.93 & 1.93 \\
\hline 0.333 & 5.99 & 2.00 & 2.06 & 2.08 & 2.14 & 2.28 & 2.28 \\
\hline 0.417 & 7.41 & 2.29 & 2.36 & 2.38 & 2.45 & 2.64 & 2.64 \\
\hline 0.500 & 8.83 & 2.59 & 2.66 & 2.69 & 2.75 & 2.99 & 2.99 \\
\hline 0.583 & 10.25 & 2.88 & 2.96 & 2.99 & 3.06 & 3.34 & 3.34 \\
\hline 0.667 & 11.66 & 3.17 & 3.26 & 3.29 & 3.36 & 3.69 & 3.69 \\
\hline 0.750 & 13.08 & 3.46 & 3.56 & 3.60 & 3.67 & 4.04 & 4.04 \\
\hline 0.833 & 14.50 & 3.76 & 3.87 & 3.90 & 3.97 & 4.40 & 4.40 \\
\hline 0.917 & 15.92 & 4.05 & 4.17 & 4.21 & 4.27 & 4.75 & 4.75 \\
\hline 1.000 & 17.34 & 4.34 & 4.47 & 4.51 & 4.58 & 5.10 & 5.10 \\
\hline 1.083 & 18.76 & 4.63 & 4.77 & 4.82 & 4.88 & 5.45 & 5.45 \\
\hline 1.167 & 20.17 & 4.92 & 5.07 & 5.12 & 5.19 & 5.80 & 5.80 \\
\hline 1.250 & 21.59 & 5.22 & 5.37 & 5.42 & 5.49 & 6.16 & 6.16 \\
\hline 1.333 & 23.01 & 5.51 & 5.68 & 5.73 & 5.79 & 6.51 & 6.51 \\
\hline 1.417 & 24.43 & 5.80 & 5.98 & 6.03 & 6.10 & 6.86 & 6.86 \\
\hline 1.500 & 25.85 & 6.09 & 6.28 & 6.34 & 6.40 & 7.21 & 7.21 \\
\hline
\end{tabular}


Table 3-1. L-Bundle and UNF Assemblies Total Surface Areas per Immersion Height

\begin{tabular}{|c|c|c|c|c|c|c|c|}
\hline $\begin{array}{c}\text { Assembly } \\
\text { Immersion } \\
\text { Depth [ft] }\end{array}$ & $\begin{array}{c}\text { MURR \& } \\
\text { L-Bundle } \\
\text { Total } \\
\text { Surface } \\
\text { Area [ft }{ }^{2} \text { ] } \\
\end{array}$ & \begin{tabular}{|c|} 
Outer SRE \\
\& L-Bundle \\
Total \\
Surface Area \\
{$\left[\mathrm{ft}^{2}\right]$}
\end{tabular} & \begin{tabular}{|c|} 
Outer \& \\
Inner SRE \& \\
L-Bundle \\
Total Surface \\
Area [ft ${ }^{2}$ ] \\
\end{tabular} & \begin{tabular}{|c|} 
HMI-Std \& \\
L-Bundle \\
Total \\
Surface \\
Area $\left[\mathrm{ft}^{2}\right]$ \\
\end{tabular} & \begin{tabular}{|c|} 
HMI-Ctrl \& \\
L-Bundle \\
Total \\
Surface \\
Area $\left[\mathrm{ft}^{2}\right]$ \\
\end{tabular} & $\begin{array}{c}\text { DR-3-1 \& } \\
\text { L-Bundle } \\
\text { Total } \\
\text { Surface } \\
\text { Area [ft }{ }^{2} \text { ] } \\
\end{array}$ & $\begin{array}{c}\text { DR-3-2 \& } \\
\text { L-Bundle } \\
\text { Total } \\
\text { Surface } \\
\text { Area [ft }{ }^{2} \text { ] } \\
\end{array}$ \\
\hline 1.583 & 27.27 & 6.39 & 6.58 & 6.64 & 6.71 & 7.56 & 7.56 \\
\hline 1.667 & 28.68 & 6.68 & 6.88 & 6.95 & 7.01 & 7.92 & 7.92 \\
\hline 1.750 & 30.10 & 6.97 & 7.18 & 7.25 & 7.32 & 8.27 & 8.27 \\
\hline 1.833 & 31.52 & 7.26 & 7.49 & 7.55 & 7.62 & 8.62 & 8.62 \\
\hline 1.917 & 32.94 & 7.56 & 7.79 & 7.86 & 7.92 & 8.97 & 8.97 \\
\hline 2.000 & 34.36 & 7.85 & 8.09 & 8.16 & 8.23 & 9.32 & 9.32 \\
\hline 2.083 & 35.77 & 8.14 & 8.39 & 8.47 & 8.53 & 9.74 & 9.68 \\
\hline 2.167 & 37.22 & 8.43 & 8.69 & 8.79 & 8.84 & 10.10 & 10.03 \\
\hline 2.250 & 37.63 & 8.72 & 8.99 & 9.23 & 9.14 & 10.51 & 10.36 \\
\hline 2.333 & 38.59 & 9.02 & 9.30 & 9.54 & 9.45 & 10.86 & 10.86 \\
\hline 2.417 & 40.01 & 9.31 & 9.60 & 9.84 & 9.74 & 11.21 & 11.21 \\
\hline 2.500 & 41.43 & 9.60 & 9.90 & 10.15 & 10.01 & 11.56 & 11.56 \\
\hline 2.583 & 42.85 & 9.89 & 10.20 & 10.45 & 10.36 & 11.91 & 11.91 \\
\hline 2.667 & 44.27 & 10.19 & 10.50 & 10.75 & 10.74 & 12.27 & 12.27 \\
\hline 2.750 & 45.68 & 10.48 & 10.80 & 11.06 & 11.07 & 12.62 & 12.62 \\
\hline 2.833 & 47.10 & 10.77 & 11.11 & 11.36 & 11.37 & 12.97 & 12.97 \\
\hline 2.917 & 48.52 & 11.06 & 11.41 & 11.67 & 11.67 & 13.32 & 13.32 \\
\hline 3.000 & 49.94 & 11.35 & 11.71 & 11.97 & 11.98 & 13.67 & 13.67 \\
\hline 3.083 & 51.36 & 11.65 & 12.01 & 12.28 & 12.28 & 14.03 & 14.03 \\
\hline 3.167 & 52.77 & 11.94 & 12.31 & 12.58 & 12.59 & 14.38 & 14.38 \\
\hline 3.250 & 54.19 & 12.23 & 12.61 & 12.88 & 12.89 & 14.73 & 14.73 \\
\hline 3.333 & 55.61 & 12.52 & 12.91 & 13.19 & 13.20 & 15.08 & 15.08 \\
\hline 3.417 & 57.03 & 12.82 & 13.22 & 13.49 & 13.50 & 15.43 & 15.43 \\
\hline 3.500 & 58.45 & 13.11 & 13.52 & 13.80 & 13.80 & 15.79 & 15.79 \\
\hline 3.583 & 59.87 & 13.40 & 13.82 & 14.10 & 14.11 & 16.14 & 16.14 \\
\hline 3.667 & 61.28 & 13.69 & 14.12 & 14.41 & 14.41 & 16.49 & 16.49 \\
\hline 3.750 & 62.70 & 13.98 & 14.42 & 14.71 & 14.72 & 16.84 & 16.84 \\
\hline 3.833 & 64.12 & 14.28 & 14.72 & 15.01 & 15.02 & 17.19 & 17.19 \\
\hline 3.917 & 65.54 & 14.57 & 15.03 & 15.32 & 15.32 & 17.55 & 17.55 \\
\hline 4.000 & 66.96 & 14.86 & 15.33 & 15.62 & 15.63 & 17.90 & 17.90 \\
\hline 4.083 & 68.37 & 15.15 & 15.63 & 15.93 & 15.93 & 18.25 & 18.25 \\
\hline 4.167 & 69.79 & 15.45 & 15.93 & 16.23 & 16.24 & 18.55 & 18.60 \\
\hline 4.250 & 71.21 & 15.74 & 16.23 & 16.54 & 16.54 & 18.92 & 18.95 \\
\hline 4.333 & 72.63 & 16.03 & 16.53 & 16.80 & 16.85 & 19.38 & 19.31 \\
\hline 4.417 & 74.08 & 16.32 & 16.84 & 17.25 & 17.15 & 19.73 & 19.60 \\
\hline 4.500 & 74.48 & 16.61 & 17.14 & 17.55 & 17.45 & 20.08 & 19.94 \\
\hline 4.583 & 75.45 & 16.91 & 17.46 & 17.86 & 17.76 & 20.43 & 20.43 \\
\hline 4.667 & 76.87 & 17.20 & 17.81 & 18.16 & 18.06 & 20.79 & 20.79 \\
\hline
\end{tabular}


Table 3-1. L-Bundle and UNF Assemblies Total Surface Areas per Immersion Height

\begin{tabular}{|c|c|c|c|c|c|c|c|}
\hline $\begin{array}{c}\text { Assembly } \\
\text { Immersion } \\
\text { Depth [ft] }\end{array}$ & $\begin{array}{c}\text { MURR \& } \\
\text { L-Bundle } \\
\text { Total } \\
\text { Surface } \\
\text { Area [ft }^{2} \text { ] } \\
\end{array}$ & \begin{tabular}{|c|} 
Outer SRE \\
\& L-Bundle \\
Total \\
Surface Area \\
{$\left[\mathrm{ft}^{2}\right]$}
\end{tabular} & \begin{tabular}{|c|} 
Outer \& \\
Inner SRE \& \\
L-Bundle \\
Total Surface \\
Area [ft $\left.{ }^{2}\right]$ \\
\end{tabular} & \begin{tabular}{|c|} 
HMI-Std \& \\
L-Bundle \\
Total \\
Surface \\
Area $\left[\mathrm{ft}^{2}\right]$ \\
\end{tabular} & \begin{tabular}{|c} 
HMI-Ctrl \& \\
L-Bundle \\
Total \\
Surface \\
Area $\left[\mathrm{ft}^{2}{ }^{2}\right]$ \\
\end{tabular} & $\begin{array}{c}\text { DR-3-1 \& } \\
\text { L-Bundle } \\
\text { Total } \\
\text { Surface } \\
{\text { Area }\left[\mathrm{ft}^{2}\right]} \\
\end{array}$ & $\begin{array}{c}\text { DR-3-2 \& } \\
\text { L-Bundle } \\
\text { Total } \\
\text { Surface } \\
\text { Area [ft }{ }^{2} \text { ] }\end{array}$ \\
\hline 4.750 & 78.28 & 17.49 & 17.49 & 18.46 & 18.37 & 21.14 & 21.14 \\
\hline 4.833 & 79.70 & 17.78 & 17.78 & 18.77 & 18.67 & 21.49 & 21.49 \\
\hline 4.917 & 81.12 & 18.08 & 18.08 & 19.07 & 18.98 & 21.84 & 21.84 \\
\hline 5.000 & 82.54 & 18.37 & 18.37 & 19.38 & 19.25 & 22.19 & 22.19 \\
\hline 5.083 & 83.96 & 18.66 & 18.66 & 19.68 & 19.57 & 22.55 & 22.55 \\
\hline 5.167 & 85.38 & 18.95 & 18.95 & 19.99 & 19.94 & 22.90 & 22.90 \\
\hline 5.250 & 86.79 & 19.25 & 19.25 & 20.29 & 20.29 & 23.25 & 23.25 \\
\hline 5.333 & 88.21 & 19.54 & 19.54 & 20.59 & 20.60 & 23.60 & 23.60 \\
\hline 5.417 & 89.63 & 19.83 & 19.83 & 20.90 & 20.90 & 23.95 & 23.95 \\
\hline 5.500 & 91.05 & 20.12 & 20.12 & 21.20 & 21.20 & 24.31 & 24.31 \\
\hline 5.583 & 92.47 & 20.41 & 20.41 & 21.51 & 21.51 & 24.66 & 24.66 \\
\hline 5.667 & 93.88 & 20.71 & 20.71 & 21.81 & 21.81 & 25.01 & 25.01 \\
\hline 5.750 & 95.30 & 21.00 & 21.00 & 22.12 & 22.12 & 25.36 & 25.36 \\
\hline 5.833 & 96.72 & 21.29 & 21.29 & 22.42 & 22.42 & 25.71 & 25.71 \\
\hline 5.917 & 98.14 & 21.58 & 21.58 & 22.72 & 22.72 & 26.07 & 26.07 \\
\hline 6.000 & 99.56 & 21.88 & 21.88 & 23.03 & 23.03 & 26.42 & 26.42 \\
\hline 6.083 & 100.98 & 22.17 & 22.17 & 23.33 & 23.33 & 26.77 & 26.77 \\
\hline 6.167 & 102.39 & 22.46 & 22.46 & 23.64 & 23.64 & 27.12 & 27.12 \\
\hline 6.250 & 103.81 & 22.75 & 22.75 & 23.94 & 23.94 & 27.47 & 27.47 \\
\hline 6.333 & 105.23 & 23.04 & 23.04 & 24.25 & 24.25 & 27.80 & 27.83 \\
\hline 6.417 & 106.65 & 23.34 & 23.34 & 24.55 & 24.55 & 28.10 & 28.18 \\
\hline 6.500 & 108.07 & 23.63 & 23.63 & 24.82 & 24.85 & 28.60 & 28.53 \\
\hline 6.583 & 109.48 & 23.92 & 23.92 & 25.26 & 25.16 & 28.95 & 28.88 \\
\hline 6.667 & 110.94 & 24.21 & 24.21 & 25.57 & 25.46 & 29.31 & 29.18 \\
\hline 6.750 & 111.34 & 24.51 & 24.51 & 25.87 & 25.77 & 29.66 & 29.53 \\
\hline 6.833 & 112.31 & 24.80 & 24.80 & 26.17 & 26.07 & 30.01 & 30.01 \\
\hline 6.917 & 113.73 & 25.09 & 25.09 & 26.48 & 26.38 & 30.36 & 30.36 \\
\hline 7.000 & 115.14 & 25.38 & 25.38 & 26.78 & 26.68 & 30.71 & 30.71 \\
\hline 7.083 & 116.56 & 25.67 & 25.67 & 27.09 & 26.98 & 31.07 & 31.07 \\
\hline 7.167 & 117.98 & 25.97 & 25.97 & 27.39 & 27.29 & 31.42 & 31.42 \\
\hline 7.250 & 119.40 & 26.26 & 26.26 & 27.70 & 27.59 & 31.77 & 31.77 \\
\hline 7.333 & 120.82 & 26.55 & 26.55 & 28.00 & 27.90 & 32.12 & 32.12 \\
\hline 7.417 & 122.24 & 26.84 & 26.84 & 28.30 & 28.21 & 32.47 & 32.47 \\
\hline 7.500 & 123.65 & 27.14 & 27.14 & 28.61 & 28.49 & 32.83 & 32.83 \\
\hline 7.583 & 125.07 & 27.43 & 27.43 & 28.91 & 28.78 & 33.18 & 33.18 \\
\hline 7.667 & 126.49 & 27.72 & 27.72 & 29.22 & 29.14 & 33.53 & 33.53 \\
\hline 7.750 & 127.91 & 28.01 & 28.01 & 29.52 & 29.52 & 33.88 & 33.88 \\
\hline 7.833 & 129.33 & 28.31 & 28.31 & 29.83 & 29.82 & 34.23 & 34.23 \\
\hline
\end{tabular}


Table 3-1. L-Bundle and UNF Assemblies Total Surface Areas per Immersion Height

\begin{tabular}{|c|c|c|c|c|c|c|c|}
\hline $\begin{array}{c}\text { Assembly } \\
\text { Immersion } \\
\text { Depth [ft] }\end{array}$ & $\begin{array}{c}\text { MURR \& } \\
\text { L-Bundle } \\
\text { Total } \\
\text { Surface } \\
\text { Area [ft }^{2} \text { ] } \\
\end{array}$ & $\begin{array}{c}\text { Outer SRE } \\
\text { \& L-Bundle } \\
\text { Total } \\
\text { Surface Area } \\
{\left[\mathrm{ft}^{2}\right]}\end{array}$ & $\begin{array}{c}\text { Outer \& } \\
\text { Inner SRE \& } \\
\text { L-Bundle } \\
\text { Total Surface } \\
\text { Area [ft }{ }^{2} \text { ] } \\
\end{array}$ & \begin{tabular}{|c|} 
HMI-Std \& \\
L-Bundle \\
Total \\
Surface \\
Area $\left[\mathrm{ft}^{2}\right]$ \\
\end{tabular} & \begin{tabular}{|c} 
HMI-Ctrl \& \\
L-Bundle \\
Total \\
Surface \\
Area $\left[\mathrm{ft}^{2}{ }^{2}\right]$ \\
\end{tabular} & $\begin{array}{c}\text { DR-3-1 \& } \\
\text { L-Bundle } \\
\text { Total } \\
\text { Surface } \\
\text { Area [ft }{ }^{2} \text { ] } \\
\end{array}$ & $\begin{array}{c}\text { DR-3-2 \& } \\
\text { L-Bundle } \\
\text { Total } \\
\text { Surface } \\
\text { Area [ft }{ }^{2} \text { ] }\end{array}$ \\
\hline 7.917 & 130.74 & 28.60 & 28.60 & 30.13 & 30.12 & 34.59 & 34.59 \\
\hline 8.000 & 132.16 & 28.89 & 28.89 & 30.43 & 30.43 & 34.94 & 34.94 \\
\hline 8.083 & 133.58 & 29.18 & 29.18 & 30.74 & 30.73 & 35.29 & 35.29 \\
\hline 8.167 & 135.00 & 29.47 & 29.47 & 31.04 & 31.04 & 35.64 & 35.64 \\
\hline 8.250 & 136.42 & 29.77 & 29.77 & 31.35 & 31.34 & 35.99 & 35.99 \\
\hline 8.333 & 137.84 & 30.06 & 30.06 & 31.65 & 31.65 & 36.35 & 36.35 \\
\hline 8.417 & 139.25 & 30.35 & 30.35 & 31.96 & 31.95 & 36.70 & 36.70 \\
\hline 8.500 & 140.67 & 30.64 & 30.64 & 32.26 & 32.25 & 37.06 & 37.05 \\
\hline 8.583 & 142.09 & 30.94 & 30.94 & 32.56 & 32.56 & 37.43 & 37.40 \\
\hline 8.667 & 143.51 & 31.23 & 31.23 & 32.83 & 32.86 & 37.83 & 37.75 \\
\hline 8.750 & 144.93 & 31.55 & 31.55 & 33.28 & 33.17 & 38.18 & 38.10 \\
\hline 8.833 & 146.35 & 31.81 & 31.81 & 33.58 & 33.47 & 38.53 & 38.46 \\
\hline 8.917 & 147.80 & 32.07 & 32.07 & 33.88 & 33.78 & 38.88 & 38.76 \\
\hline 9.000 & 148.16 & 32.36 & 32.36 & 34.19 & 34.08 & 39.23 & 39.11 \\
\hline 9.083 & 148.37 & 32.60 & 32.60 & 34.49 & 34.38 & 39.59 & 39.57 \\
\hline 9.167 & 148.59 & 32.84 & 32.84 & 34.80 & 34.69 & 39.94 & 39.92 \\
\hline 9.250 & 148.80 & & & 35.10 & 34.99 & 40.29 & 40.27 \\
\hline 9.333 & 149.02 & & & 35.41 & 35.30 & 40.64 & 40.62 \\
\hline 9.417 & 149.23 & & & 35.71 & 35.60 & 40.99 & 40.97 \\
\hline 9.500 & 149.45 & & & 36.01 & 35.91 & 41.35 & 41.32 \\
\hline 9.583 & 149.66 & & & 36.32 & 36.21 & 41.70 & 41.68 \\
\hline 9.667 & 149.88 & & & 36.62 & 36.51 & 42.05 & 42.03 \\
\hline 9.750 & 150.10 & & & 36.93 & 36.82 & 42.40 & 42.38 \\
\hline 9.833 & 150.31 & & & 37.23 & 37.12 & 42.75 & 42.73 \\
\hline 9.917 & 150.53 & & & 37.54 & 37.43 & 43.11 & 43.08 \\
\hline 10.000 & 150.74 & & & 37.84 & 37.72 & 43.46 & 43.44 \\
\hline 10.083 & 150.96 & & & 38.14 & 37.99 & 43.81 & 43.79 \\
\hline 10.167 & 151.17 & & & 38.45 & & 44.16 & 44.14 \\
\hline 10.250 & 151.39 & & & 38.75 & & 44.51 & 44.49 \\
\hline 10.333 & 151.60 & & & 39.06 & & & 44.84 \\
\hline 10.417 & 151.82 & & & 39.36 & & & 45.20 \\
\hline 10.500 & 152.03 & & & 39.67 & & & 45.55 \\
\hline 10.583 & 152.25 & & & 39.97 & & & 45.90 \\
\hline 10.667 & 152.46 & & & 40.27 & & & 46.25 \\
\hline 10.750 & 152.68 & & & 40.58 & & & 46.60 \\
\hline 10.833 & 152.89 & & & 40.85 & & & 46.96 \\
\hline 10.917 & 153.11 & & & & & & \\
\hline 11.000 & 153.33 & & & & & & \\
\hline
\end{tabular}


SRNL-STI-2012-00279

Revision 1

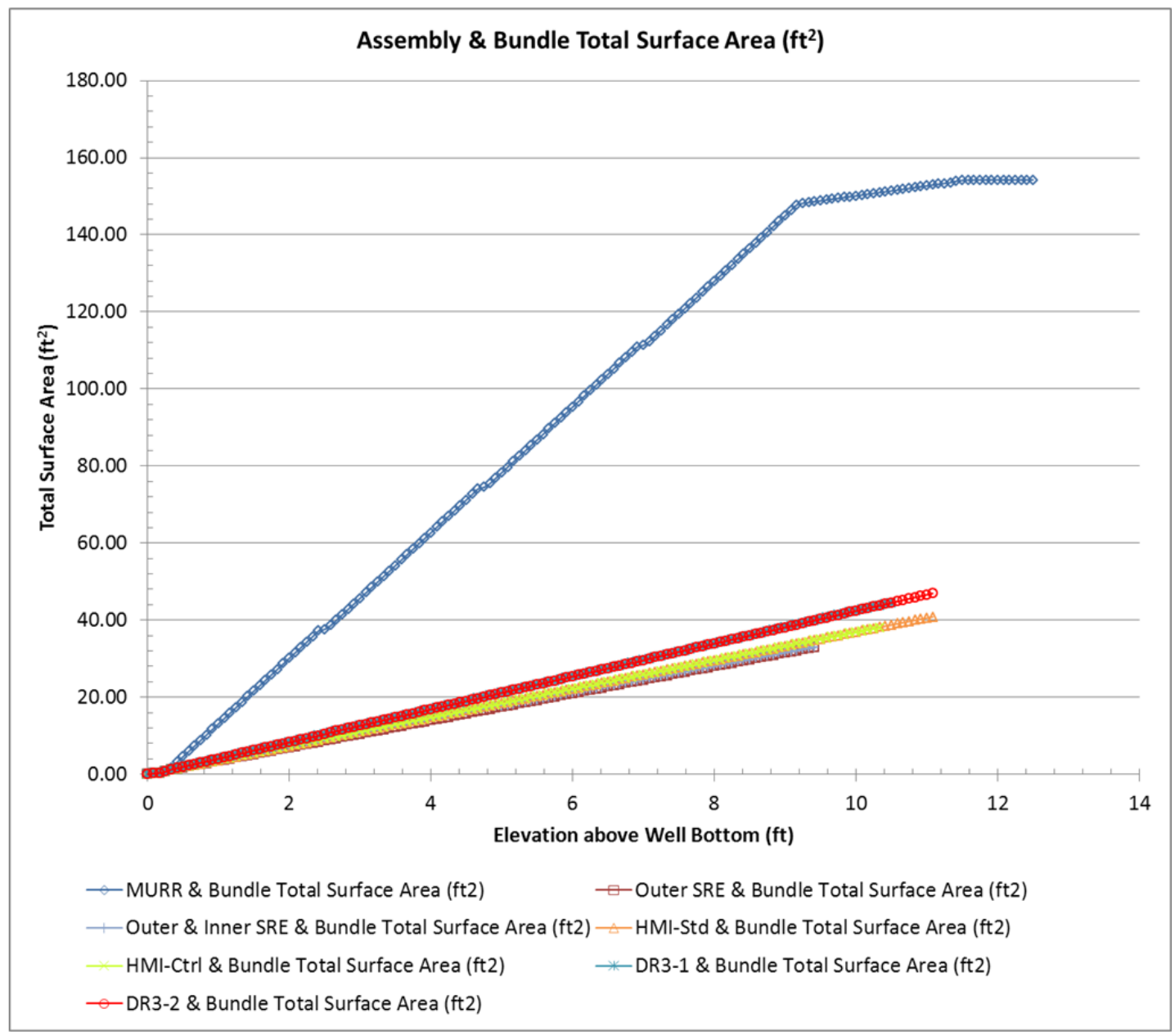

Figure 3-1. L-Bundle and Various UNF Assemblies Total Surface Areas per Immersion Height

Table 3-2. L-Bundle and UNF Assemblies Surface Areas per Immersion Height for Off-gas Calculations

\begin{tabular}{|c|c|c|c|c|c|c|c|}
\hline $\begin{array}{c}\text { Assembly } \\
\text { Immersion } \\
\text { Depth [ft] }\end{array}$ & $\begin{array}{c}\text { L-Bundle } \\
\text { Outer } \\
\text { Surface } \\
\text { Area }\left(\mathbf{f t}^{2}\right) \\
\end{array}$ & $\begin{array}{c}\text { Outer } \\
\text { SRE } \\
\text { Surface } \\
\text { Area }\left(\mathbf{f t}^{2}\right) \\
\end{array}$ & $\begin{array}{c}\text { Inner SRE } \\
\text { Surface } \\
\text { Area }\left(\mathbf{f t}^{2}\right)\end{array}$ & $\begin{array}{c}\text { HMI-Std } \\
\text { Surface } \\
\text { Area }\left(\mathrm{ft}^{2}{ }^{2}\right)\end{array}$ & $\begin{array}{c}\text { HMI-Ctrl } \\
\text { Surface } \\
\text { Area }\left(\mathbf{f t}^{2}\right)\end{array}$ & $\begin{array}{c}\text { DR-3-1 } \\
\text { Surface } \\
\text { Area }\left(\mathrm{ft}^{2}\right)\end{array}$ & $\begin{array}{c}\text { DR-3-2 } \\
\text { Surface } \\
\text { Area }\left(\mathrm{ft}^{2}\right)\end{array}$ \\
\hline 0.000 & 0.44 & 0.00 & 0.00 & 0.00 & 0.00 & 0.00 & 0.00 \\
\hline 0.083 & 0.67 & 0.13 & 0.03 & 0.17 & 0.17 & 0.24 & 0.24 \\
\hline 0.167 & 0.78 & 0.21 & 0.04 & 0.26 & 0.33 & 0.37 & 0.37 \\
\hline 0.250 & 0.89 & 0.29 & 0.04 & 0.35 & 0.42 & 0.51 & 0.51 \\
\hline 0.333 & 1.00 & 0.36 & 0.05 & 0.44 & 0.50 & 0.65 & 0.65 \\
\hline 0.417 & 1.11 & 0.44 & 0.06 & 0.53 & 0.59 & 0.78 & 0.78 \\
\hline 0.500 & 1.22 & 0.52 & 0.07 & 0.61 & 0.68 & 0.92 & 0.92 \\
\hline 0.583 & 1.33 & 0.59 & 0.08 & 0.70 & 0.77 & 1.05 & 1.05 \\
\hline 0.667 & 1.44 & 0.67 & 0.09 & 0.79 & 0.86 & 1.19 & 1.19 \\
\hline 0.750 & 1.55 & 0.74 & 0.10 & 0.88 & 0.95 & 1.33 & 1.33 \\
\hline 0.833 & 1.65 & 0.82 & 0.11 & 0.97 & 1.03 & 1.46 & 1.46 \\
\hline
\end{tabular}


SRNL-STI-2012-00279

Revision 1

Table 3-2. L-Bundle and UNF Assemblies Surface Areas per Immersion Height for Off-gas Calculations

\begin{tabular}{|c|c|c|c|c|c|c|c|}
\hline $\begin{array}{c}\text { Assembly } \\
\text { Immersion } \\
\text { Depth [ft] }\end{array}$ & $\begin{array}{c}\text { L-Bundle } \\
\text { Outer } \\
\text { Surface } \\
\left.\text { Area (ft }{ }^{2}\right)\end{array}$ & $\begin{array}{c}\text { Outer } \\
\text { SRE } \\
\text { Surface } \\
\text { Area }\left(\mathbf{f t}^{2}\right)\end{array}$ & $\begin{array}{c}\text { Inner SRE } \\
\text { Surface } \\
\text { Area }\left(\mathbf{f t}^{2}\right)\end{array}$ & $\begin{array}{c}\text { HMI-Std } \\
\text { Surface } \\
\text { Area }\left(\mathbf{f t}^{2}\right)\end{array}$ & $\begin{array}{c}\text { HMI-Ctrl } \\
\text { Surface } \\
\text { Area }\left(\mathbf{f t}^{2}\right)\end{array}$ & $\begin{array}{c}\text { DR-3-1 } \\
\text { Surface } \\
\text { Area }\left(\mathbf{f t}^{2}\right)\end{array}$ & $\begin{array}{c}\text { DR-3-2 } \\
\text { Surface } \\
\text { Area }\left(\mathbf{f t}^{2}\right)\end{array}$ \\
\hline 0.917 & 1.76 & 0.90 & 0.12 & 1.06 & 1.12 & 1.60 & 1.60 \\
\hline 1.000 & 1.87 & 0.97 & 0.13 & 1.14 & 1.21 & 1.73 & 1.73 \\
\hline 1.083 & 1.98 & 1.05 & 0.14 & 1.23 & 1.30 & 1.87 & 1.87 \\
\hline 1.167 & 2.09 & 1.13 & 0.15 & 1.32 & 1.39 & 2.01 & 2.01 \\
\hline 1.250 & 2.20 & 1.20 & 0.16 & 1.41 & 1.48 & 2.14 & 2.14 \\
\hline 1.333 & 2.31 & 1.28 & 0.17 & 1.50 & 1.56 & 2.28 & 2.28 \\
\hline 1.417 & 2.42 & 1.36 & 0.18 & 1.59 & 1.65 & 2.41 & 2.41 \\
\hline 1.500 & 2.53 & 1.43 & 0.19 & 1.67 & 1.74 & 2.55 & 2.55 \\
\hline 1.583 & 2.64 & 1.51 & 0.19 & 1.76 & 1.83 & 2.69 & 2.69 \\
\hline 1.667 & 2.75 & 1.58 & 0.20 & 1.85 & 1.92 & 2.82 & 2.82 \\
\hline 1.750 & 2.85 & 1.66 & 0.21 & 1.94 & 2.01 & 2.96 & 2.96 \\
\hline 1.833 & 2.96 & 1.74 & 0.22 & 2.03 & 2.09 & 3.09 & 3.09 \\
\hline 1.917 & 3.07 & 1.81 & 0.23 & 2.12 & 2.18 & 3.23 & 3.23 \\
\hline 2.000 & 3.18 & 1.89 & 0.24 & 2.20 & 2.27 & 3.37 & 3.37 \\
\hline 2.083 & 3.29 & 1.97 & 0.25 & 2.29 & 2.36 & 3.56 & 3.50 \\
\hline 2.167 & 3.40 & 2.04 & 0.26 & 2.40 & 2.45 & 3.71 & 3.64 \\
\hline 2.250 & 3.51 & 2.12 & 0.27 & 2.63 & 2.54 & 3.90 & 3.75 \\
\hline 2.333 & 3.62 & 2.19 & 0.28 & 2.72 & 2.62 & 4.04 & 4.04 \\
\hline 2.417 & 3.73 & 2.27 & 0.29 & 2.80 & 2.71 & 4.17 & 4.17 \\
\hline 2.500 & 3.84 & 2.35 & 0.30 & 2.89 & 2.76 & 4.31 & 4.31 \\
\hline 2.583 & 3.95 & 2.42 & 0.31 & 2.98 & 2.89 & 4.45 & 4.45 \\
\hline 2.667 & 4.05 & 2.50 & 0.32 & 3.07 & 3.05 & 4.58 & 4.58 \\
\hline 2.750 & 4.16 & 2.58 & 0.33 & 3.16 & 3.16 & 4.72 & 4.72 \\
\hline 2.833 & 4.27 & 2.65 & 0.34 & 3.25 & 3.25 & 4.85 & 4.85 \\
\hline 2.917 & 4.38 & 2.73 & 0.34 & 3.33 & 3.34 & 4.99 & 4.99 \\
\hline 3.000 & 4.49 & 2.81 & 0.35 & 3.42 & 3.43 & 5.13 & 5.13 \\
\hline 3.083 & 4.60 & 2.88 & 0.36 & 3.51 & 3.52 & 5.26 & 5.26 \\
\hline 3.167 & 4.71 & 2.96 & 0.37 & 3.60 & 3.61 & 5.40 & 5.40 \\
\hline 3.250 & 4.82 & 3.03 & 0.38 & 3.69 & 3.69 & 5.53 & 5.53 \\
\hline 3.333 & 4.93 & 3.11 & 0.39 & 3.78 & 3.78 & 5.67 & 5.67 \\
\hline 3.417 & 5.04 & 3.19 & 0.40 & 3.87 & 3.87 & 5.81 & 5.81 \\
\hline 3.500 & 5.15 & 3.26 & 0.41 & 3.95 & 3.96 & 5.94 & 5.94 \\
\hline 3.583 & 5.25 & 3.34 & 0.42 & 4.04 & 4.05 & 6.08 & 6.08 \\
\hline 3.667 & 5.36 & 3.42 & 0.43 & 4.13 & 4.14 & 6.21 & 6.21 \\
\hline 3.750 & 5.47 & 3.49 & 0.44 & 4.22 & 4.22 & 6.35 & 6.35 \\
\hline 3.833 & 5.58 & 3.57 & 0.45 & 4.31 & 4.31 & 6.49 & 6.49 \\
\hline 3.917 & 5.69 & 3.65 & 0.46 & 4.40 & 4.40 & 6.62 & 6.62 \\
\hline 4.000 & 5.80 & 3.72 & 0.47 & 4.48 & 4.49 & 6.76 & 6.76 \\
\hline
\end{tabular}


SRNL-STI-2012-00279

Revision 1

Table 3-2. L-Bundle and UNF Assemblies Surface Areas per Immersion Height for Off-gas Calculations

\begin{tabular}{|c|c|c|c|c|c|c|c|}
\hline $\begin{array}{c}\text { Assembly } \\
\text { Immersion } \\
\text { Depth [ft] }\end{array}$ & $\begin{array}{c}\text { L-Bundle } \\
\text { Outer } \\
\text { Surface } \\
\left.\text { Area (ft }{ }^{2}\right)\end{array}$ & $\begin{array}{c}\text { Outer } \\
\text { SRE } \\
\text { Surface } \\
\text { Area }\left(\mathbf{f t}^{2}\right)\end{array}$ & $\begin{array}{c}\text { Inner SRE } \\
\text { Surface } \\
\text { Area }\left(\mathbf{f t}^{2}\right)\end{array}$ & $\begin{array}{c}\text { HMI-Std } \\
\text { Surface } \\
\text { Area }\left(\mathbf{f t}^{2}\right)\end{array}$ & $\begin{array}{c}\text { HMI-Ctrl } \\
\text { Surface } \\
\text { Area }\left(\mathbf{f t}^{2}\right)\end{array}$ & $\begin{array}{c}\text { DR-3-1 } \\
\text { Surface } \\
\text { Area }\left(\mathbf{f t}^{2}\right)\end{array}$ & $\begin{array}{c}\text { DR-3-2 } \\
\text { Surface } \\
\text { Area }\left(\mathbf{f t}^{2}\right)\end{array}$ \\
\hline 4.083 & 5.91 & 3.80 & 0.48 & 4.57 & 4.58 & 6.89 & 6.89 \\
\hline 4.167 & 6.02 & 3.87 & 0.49 & 4.66 & 4.67 & 6.98 & 7.03 \\
\hline 4.250 & 6.13 & 3.95 & 0.49 & 4.75 & 4.75 & 7.13 & 7.17 \\
\hline 4.333 & 6.24 & 4.03 & 0.50 & 4.80 & 4.84 & 7.38 & 7.30 \\
\hline 4.417 & 6.35 & 4.10 & 0.51 & 5.03 & 4.93 & 7.51 & 7.39 \\
\hline 4.500 & 6.45 & 4.18 & 0.52 & 5.12 & 5.02 & 7.65 & 7.51 \\
\hline 4.583 & 6.56 & 4.26 & 0.55 & 5.21 & 5.11 & 7.78 & 7.78 \\
\hline 4.667 & 6.67 & 4.33 & 0.61 & 5.29 & 5.20 & 7.92 & 7.92 \\
\hline 4.750 & 6.78 & 4.41 & 0.00 & 5.38 & 5.29 & 8.06 & 8.06 \\
\hline 4.833 & 6.89 & 4.49 & 0.00 & 5.47 & 5.37 & 8.19 & 8.19 \\
\hline 4.917 & 7.00 & 4.56 & 0.00 & 5.56 & 5.46 & 8.33 & 8.33 \\
\hline 5.000 & 7.11 & 4.64 & 0.00 & 5.65 & 5.52 & 8.46 & 8.46 \\
\hline 5.083 & 7.22 & 4.71 & 0.00 & 5.74 & 5.62 & 8.60 & 8.60 \\
\hline 5.167 & 7.33 & 4.79 & 0.00 & 5.82 & 5.78 & 8.74 & 8.74 \\
\hline 5.250 & 7.44 & 4.87 & 0.00 & 5.91 & 5.91 & 8.87 & 8.87 \\
\hline 5.333 & 7.55 & 4.94 & 0.00 & 6.00 & 6.00 & 9.01 & 9.01 \\
\hline 5.417 & 7.65 & 5.02 & 0.00 & 6.09 & 6.09 & 9.14 & 9.14 \\
\hline 5.500 & 7.76 & 5.10 & 0.00 & 6.18 & 6.18 & 9.28 & 9.28 \\
\hline 5.583 & 7.87 & 5.17 & 0.00 & 6.27 & 6.27 & 9.42 & 9.42 \\
\hline 5.667 & 7.98 & 5.25 & 0.00 & 6.35 & 6.35 & 9.55 & 9.55 \\
\hline 5.750 & 8.09 & 5.33 & 0.00 & 6.44 & 6.44 & 9.69 & 9.69 \\
\hline 5.833 & 8.20 & 5.40 & 0.00 & 6.53 & 6.53 & 9.82 & 9.82 \\
\hline 5.917 & 8.31 & 5.48 & 0.00 & 6.62 & 6.62 & 9.96 & 9.96 \\
\hline 6.000 & 8.42 & 5.55 & 0.00 & 6.71 & 6.71 & 10.10 & 10.10 \\
\hline 6.083 & 8.53 & 5.63 & 0.00 & 6.80 & 6.80 & 10.23 & 10.23 \\
\hline 6.167 & 8.64 & 5.71 & 0.00 & 6.88 & 6.89 & 10.37 & 10.37 \\
\hline 6.250 & 8.75 & 5.78 & 0.00 & 6.97 & 6.97 & 10.50 & 10.50 \\
\hline 6.333 & 8.85 & 5.86 & 0.00 & 7.06 & 7.06 & 10.62 & 10.64 \\
\hline 6.417 & 8.96 & 5.94 & 0.00 & 7.15 & 7.15 & 10.70 & 10.78 \\
\hline 6.500 & 9.07 & 6.01 & 0.00 & 7.20 & 7.24 & 10.99 & 10.91 \\
\hline 6.583 & 9.18 & 6.09 & 0.00 & 7.43 & 7.33 & 11.12 & 11.05 \\
\hline 6.667 & 9.29 & 6.17 & 0.00 & 7.52 & 7.42 & 11.26 & 11.13 \\
\hline 6.750 & 9.40 & 6.24 & 0.00 & 7.61 & 7.50 & 11.39 & 11.26 \\
\hline 6.833 & 9.51 & 6.32 & 0.00 & 7.69 & 7.59 & 11.53 & 11.53 \\
\hline 6.917 & 9.62 & 6.39 & 0.00 & 7.78 & 7.68 & 11.67 & 11.67 \\
\hline 7.000 & 9.73 & 6.47 & 0.00 & 7.87 & 7.77 & 11.80 & 11.80 \\
\hline 7.083 & 9.84 & 6.55 & 0.00 & 7.96 & 7.86 & 11.94 & 11.94 \\
\hline 7.167 & 9.95 & 6.62 & 0.00 & 8.05 & 7.95 & 12.07 & 12.07 \\
\hline
\end{tabular}


SRNL-STI-2012-00279

Revision 1

Table 3-2. L-Bundle and UNF Assemblies Surface Areas per Immersion Height for Off-gas Calculations

\begin{tabular}{|c|c|c|c|c|c|c|c|}
\hline $\begin{array}{c}\text { Assembly } \\
\text { Immersion } \\
\text { Depth [ft] }\end{array}$ & $\begin{array}{c}\text { L-Bundle } \\
\text { Outer } \\
\text { Surface } \\
\left.\text { Area (ft }{ }^{2}\right)\end{array}$ & $\begin{array}{c}\text { Outer } \\
\text { SRE } \\
\text { Surface } \\
\text { Area }\left(\mathbf{f t}^{2}\right)\end{array}$ & $\begin{array}{c}\text { Inner SRE } \\
\text { Surface } \\
\text { Area }\left(\mathbf{f t}^{2}\right)\end{array}$ & $\begin{array}{c}\text { HMI-Std } \\
\text { Surface } \\
\text { Area }\left(\mathbf{f t}^{2}\right)\end{array}$ & $\begin{array}{c}\text { HMI-Ctrl } \\
\text { Surface } \\
\text { Area }\left(\mathbf{f t}^{2}\right)\end{array}$ & $\begin{array}{c}\text { DR-3-1 } \\
\text { Surface } \\
\text { Area }\left(\mathbf{f t}^{2}\right)\end{array}$ & $\begin{array}{c}\text { DR-3-2 } \\
\text { Surface } \\
\text { Area }\left(\mathbf{f t}^{2}\right)\end{array}$ \\
\hline 7.250 & 10.05 & 6.70 & 0.00 & 8.14 & 8.03 & 12.21 & 12.21 \\
\hline 7.333 & 10.16 & 6.78 & 0.00 & 8.22 & 8.12 & 12.35 & 12.35 \\
\hline 7.417 & 10.27 & 6.85 & 0.00 & 8.31 & 8.22 & 12.48 & 12.48 \\
\hline 7.500 & 10.38 & 6.93 & 0.00 & 8.40 & 8.28 & 12.62 & 12.62 \\
\hline 7.583 & 10.49 & 7.01 & 0.00 & 8.49 & 8.35 & 12.75 & 12.75 \\
\hline 7.667 & 10.60 & 7.08 & 0.00 & 8.58 & 8.51 & 12.89 & 12.89 \\
\hline 7.750 & 10.71 & 7.16 & 0.00 & 8.67 & 8.66 & 13.03 & 13.03 \\
\hline 7.833 & 10.82 & 7.23 & 0.00 & 8.75 & 8.75 & 13.16 & 13.16 \\
\hline 7.917 & 10.93 & 7.31 & 0.00 & 8.84 & 8.84 & 13.30 & 13.30 \\
\hline 8.000 & 11.04 & 7.39 & 0.00 & 8.93 & 8.93 & 13.43 & 13.43 \\
\hline 8.083 & 11.15 & 7.46 & 0.00 & 9.02 & 9.02 & 13.57 & 13.57 \\
\hline 8.167 & 11.25 & 7.54 & 0.00 & 9.11 & 9.10 & 13.71 & 13.71 \\
\hline 8.250 & 11.36 & 7.62 & 0.00 & 9.20 & 9.19 & 13.84 & 13.84 \\
\hline 8.333 & 11.47 & 7.69 & 0.00 & 9.29 & 9.28 & 13.98 & 13.98 \\
\hline 8.417 & 11.58 & 7.77 & 0.00 & 9.37 & 9.37 & 14.12 & 14.12 \\
\hline 8.500 & 11.69 & 7.85 & 0.00 & 9.46 & 9.46 & 14.26 & 14.25 \\
\hline 8.583 & 11.80 & 7.92 & 0.00 & 9.55 & 9.55 & 14.41 & 14.39 \\
\hline 8.667 & 11.91 & 8.00 & 0.00 & 9.60 & 9.63 & 14.60 & 14.52 \\
\hline 8.750 & 12.02 & 8.11 & 0.00 & 9.83 & 9.72 & 14.73 & 14.66 \\
\hline 8.833 & 12.13 & 8.15 & 0.00 & 9.92 & 9.81 & 14.87 & 14.80 \\
\hline 8.917 & 12.24 & 8.19 & 0.00 & 10.01 & 9.90 & 15.00 & 14.88 \\
\hline 9.000 & 12.35 & 8.27 & 0.00 & 10.10 & 9.99 & 15.14 & 15.02 \\
\hline 9.083 & 12.45 & 8.29 & 0.00 & 10.18 & 10.08 & 15.28 & 15.26 \\
\hline 9.167 & 12.56 & 8.32 & 0.00 & 10.27 & 10.16 & 15.41 & 15.39 \\
\hline 9.250 & 12.67 & & & 10.36 & 10.25 & 15.55 & 15.53 \\
\hline 9.333 & 12.78 & & & 10.45 & 10.34 & 15.68 & 15.66 \\
\hline 9.417 & 12.89 & & & 10.54 & 10.43 & 15.82 & 15.80 \\
\hline 9.500 & 13.00 & & & 10.63 & 10.52 & 15.96 & 15.94 \\
\hline 9.583 & 13.11 & & & 10.71 & 10.61 & 16.09 & 16.07 \\
\hline 9.667 & 13.22 & & & 10.80 & 10.69 & 16.23 & 16.21 \\
\hline 9.750 & 13.33 & & & 10.89 & 10.78 & 16.37 & 16.34 \\
\hline 9.833 & 13.44 & & & 10.98 & 10.87 & 16.50 & 16.48 \\
\hline 9.917 & 13.54 & & & 11.07 & 10.96 & 16.64 & 16.62 \\
\hline 10.000 & 13.65 & & & 11.16 & 11.04 & 16.77 & 16.75 \\
\hline 10.083 & 13.76 & & & 11.24 & 11.09 & 16.91 & 16.89 \\
\hline 10.167 & 13.87 & & & 11.33 & & 17.05 & 17.02 \\
\hline 10.250 & 13.98 & & & 11.42 & & 17.18 & 17.16 \\
\hline 10.333 & 14.09 & & & 11.51 & & 17.32 & 17.30 \\
\hline
\end{tabular}


Table 3-2. L-Bundle and UNF Assemblies Surface Areas per Immersion Height for Off-gas Calculations

\begin{tabular}{|c|c|c|c|c|c|c|c|}
\hline $\begin{array}{c}\text { Assembly } \\
\text { Immersion } \\
\text { Depth [ft] }\end{array}$ & $\begin{array}{c}\text { L-Bundle } \\
\text { Outer } \\
\text { Surface } \\
\text { Area }\left(\mathbf{f t}^{2}\right) \\
\end{array}$ & $\begin{array}{c}\text { Outer } \\
\text { SRE } \\
\text { Surface } \\
\text { Area }\left(\mathbf{f t}^{2}\right) \\
\end{array}$ & $\begin{array}{c}\text { Inner SRE } \\
\text { Surface } \\
\text { Area }\left(\mathbf{f t}^{2}{ }^{2}\right)\end{array}$ & $\begin{array}{c}\text { HMI-Std } \\
\text { Surface } \\
\text { Area }\left(\mathrm{ft}^{2}{ }^{2}\right)\end{array}$ & $\begin{array}{c}\text { HMI-Ctrl } \\
\text { Surface } \\
\text { Area }\left(\mathrm{ftt}^{2}\right)\end{array}$ & $\begin{array}{c}\text { DR-3-1 } \\
\text { Surface } \\
\text { Area }\left(\mathbf{f t}^{2}\right)\end{array}$ & $\begin{array}{c}\text { DR-3-2 } \\
\text { Surface } \\
\text { Area }\left(\mathrm{ft}^{2}\right)\end{array}$ \\
\hline 10.417 & 14.20 & & & 11.60 & & 17.45 & 17.43 \\
\hline 10.500 & 14.31 & & & 11.69 & & 17.59 & 17.57 \\
\hline 10.583 & 14.42 & & & 11.77 & & 17.67 & 17.71 \\
\hline 10.667 & 14.53 & & & 11.86 & & 17.83 & 17.84 \\
\hline 10.750 & 14.64 & & & 11.95 & & & 17.98 \\
\hline 10.833 & 14.74 & & & 12.00 & & & 18.11 \\
\hline 10.917 & 14.85 & & & & & & 18.25 \\
\hline 11.000 & 14.97 & & & & & & 18.39 \\
\hline
\end{tabular}

\subsection{Gas Generation}

To complement the off-gas generation calculations based on the surface area of the UNF and prior literature, a series of dissolution experiments were performed. The gas generated during the dissolution of thorium has been reported in several previous reports. ${ }^{35,36}$ In a report by Karraker, dissolution of Th metal in $10 \mathrm{M} \mathrm{HNO}_{3}$ and $0.05 \mathrm{M}$ fluoride results in $\mathrm{H}_{2}$ gas generated at $3.60 \%$ by volume. In a later symposium report ${ }^{36}$, dissolution of Th metal in $13 \mathrm{M} \mathrm{HNO}_{3}$ and $0.05 \mathrm{M}$ fluoride is reported to generate $\mathrm{H}_{2}$ gas at $2.50 \%$ by volume. These data points are at the upper bounds desired for the upcoming dissolution campaigns. The previous work also was performed without any competing dissolution reactions. One potential complication for the upcoming campaign is the large quantities of aluminum present from the $\mathrm{HA} / \mathrm{LU}$ fuel as well as the L-Bundles containing the used fuels. The aluminum complicates the dissolution in two main areas: 1) hydrogen generation during dissolution and 2) aluminum complexation of the fluoride necessary in the dissolution of the Th metal. Experimental conditions are listed in Table 2-1 and results are listed in Table 3-3.

Thorium metal dissolution is similar to $\mathrm{Pu}$ and $\mathrm{U}$ in that a thin oxide coating forms that protects the metal from further oxidation at ambient temperatures. After the oxide film is breached at any point(s), several different metal $\mathrm{HNO}_{3}$ reactions can take place to dissolve the thorium or oxidize it to $\mathrm{ThO}_{2}$. Fluoride in solution first attacks the oxide to form an oxyfluoride which can then be dissolved by $\mathrm{HNO}_{3}$. The following Equations were derived from Karraker's work in which HF was used. ${ }^{35}$ Current campaigns will use KF.

$$
\begin{aligned}
& \mathrm{ThO}_{2}+2 \mathrm{HF} \rightarrow \mathrm{ThOF}_{2}+\mathrm{H}_{2} \mathrm{O} \\
& \mathrm{ThOF}_{2}+4 \mathrm{HNO}_{3} \rightarrow \mathrm{Th}\left(\mathrm{NO}_{3}\right)_{4}+2 \mathrm{HF}+2 \mathrm{H}_{2} \mathrm{O}
\end{aligned}
$$

The other possible reactions of Th metal are shown in the following Equations, using different amounts of acid with only one reaction requiring as much as six moles of acid per mole of metal.

$$
\begin{aligned}
& \mathrm{Th}+6 \mathrm{HNO}_{3} \rightarrow \mathrm{Th}\left(\mathrm{NO}_{3}\right)_{4}+\mathrm{NO}+\mathrm{NO}_{2}+3 \mathrm{H}_{2} \mathrm{O} \\
& 3 \mathrm{Th}+16 \mathrm{HNO}_{3} \rightarrow 3 \mathrm{Th}\left(\mathrm{NO}_{3}\right)_{4}+4 \mathrm{NO}+4 \mathrm{H}_{2} \mathrm{O} \\
& \mathrm{Th}+4 \mathrm{HNO}_{3} \rightarrow \mathrm{Th}\left(\mathrm{NO}_{3}\right)_{4}+2 \mathrm{H}_{2} \\
& \mathrm{Th}+4 \mathrm{HNO}_{3} \rightarrow \mathrm{ThO}_{2}+4 \mathrm{NO}_{2}+2 \mathrm{H}_{2} \mathrm{O}
\end{aligned}
$$


Equation 45 is a minor reaction based on Karraker's report where very little $\mathrm{NO}_{2}$ was measured in the offgas. In the current dissolutions of thorium (Th) in $4 \mathrm{M}, 7 \mathrm{M}$ and $10 \mathrm{M}$ nitric acid, little or no color was observed meaning that little $\mathrm{NO}_{2} / \mathrm{N}_{2} \mathrm{O}_{4}$ gas was evolved from the solution. When $\mathrm{Al}$ was dissolved alone, the gas evolved was the dark red color of $\mathrm{NO}_{2} / \mathrm{N}_{2} \mathrm{O}_{4}$ gas, see Figure 3-2.

Equation 46 appears to be the main reaction occurring in Th dissolution. Karraker performed dissolutions with 10-190 grams of Th in $10 \mathrm{M} \mathrm{HNO}_{3}$ with $0.1 \mathrm{M} \mathrm{HF}$. The ratio of moles of acid to moles of metal dissolved by Equation 46 is 5.33 while Karraker observed an average ratio of $5.22 \pm 0.22$ with NO being the primary component. This value lies within the uncertainty of his experiments, but does not exclude other reactions lowering the ratio.

Equation 47 also occurs to some extent since Karraker observed $\mathrm{H}_{2}$ gas in his work. The quantity of $\mathrm{H}_{2}$ produced might be expected to increase slightly as the oxidizing potential of the acid concentration decreases and due to a higher percentage of the acid being ionized at the lower acid concentration. However, as the acid concentration decreases the reaction rate decreases as well so the total volume of gas generated per minute decreases so that dilution in the off-gas system may be possible for any small increase in $\mathrm{H}_{2}$ gas generation.

Karraker reported that the off-gas composition for his experiments was 85 vol \% NO, 7.6 vol $\% \mathrm{~N}_{2}$, $3.5 \mathrm{vol} \% \mathrm{H}_{2}, 2.1 \mathrm{vol} \% \mathrm{H}_{2} \mathrm{O}$ with less than 1 vol \% each of $\mathrm{NO}_{2}, \mathrm{~N}_{2} \mathrm{O}$ and $\mathrm{O}_{2}$ during his dissolutions of Th in $10 \mathrm{M} \mathrm{HNO}_{3}$ with $0.1 \mathrm{M} \mathrm{HF}$. Nitrogen and oxygen are most likely due to residual air in the samples analyzed in the experiments. That conclusion is based on each being below the detectability limit of the analytical method so it is not known for certain about the last three gases except that the three gases total the remaining $1.8 \mathrm{vol} \%$ of the gas composition. If the gas composition is calculated without $\mathrm{N}_{2}$, the percentages become 92 vol $\% \mathrm{NO}, 3.8$ vol $\% \mathrm{H}_{2}, 2.3$ vol $\% \mathrm{H}_{2} \mathrm{O}$ with less than 1.9 vol $\%$ each of $\mathrm{NO}_{2}$, $\mathrm{N}_{2} \mathrm{O}$, and $\mathrm{O}_{2}$. The amount of $\mathrm{H}_{2} \mathrm{O}$ in the gases is not what was produced in the reaction since much of the water would condense and drain back to the reaction vessel as during plant dissolving. However, the percentages of $\mathrm{NO}$ and $\mathrm{H}_{2}$ in the off-gas are an estimate based on the contributions from Equations 46 and 47 with all other reactions contributing less than $2 \%$ to the gas volume.

Equation 48, which produces Th oxide, is a minor reaction which would be expected to occur to a slightly higher percentage at low acid concentrations due to the oxidizing potential being lower and the percent of free $\mathrm{H}^{+}$concentration being higher. However, Orth in his summary paper ${ }^{37}$ on Th processing stated that some undissolved $\mathrm{ThO}_{2}$ was present after dissolution of the metal. The dissolution rate was 4 tons per day of thorium so any of the protective layer of $\mathrm{ThO}_{2}$ would be expected to accumulate in the dissolver with some being transferred out with the solution going to solvent extraction. This campaign has less than $2 \mathrm{MT}$ of Th and will not go through solvent extraction.

Since there are multiple reactions that can occur in the solution, changes in the ratio of $\mathrm{HNO}_{3}$ consumed per mole of Th metal dissolved should be expected to be observed even at the same acid concentration, but especially as the $\mathrm{HNO}_{3}$ concentration changes. Additional dissolution tests are being proposed to clarify whether the $\mathrm{H}_{2}$ gas increases as acid concentration decreases and whether any increase is still within the safe limits for dilution. 


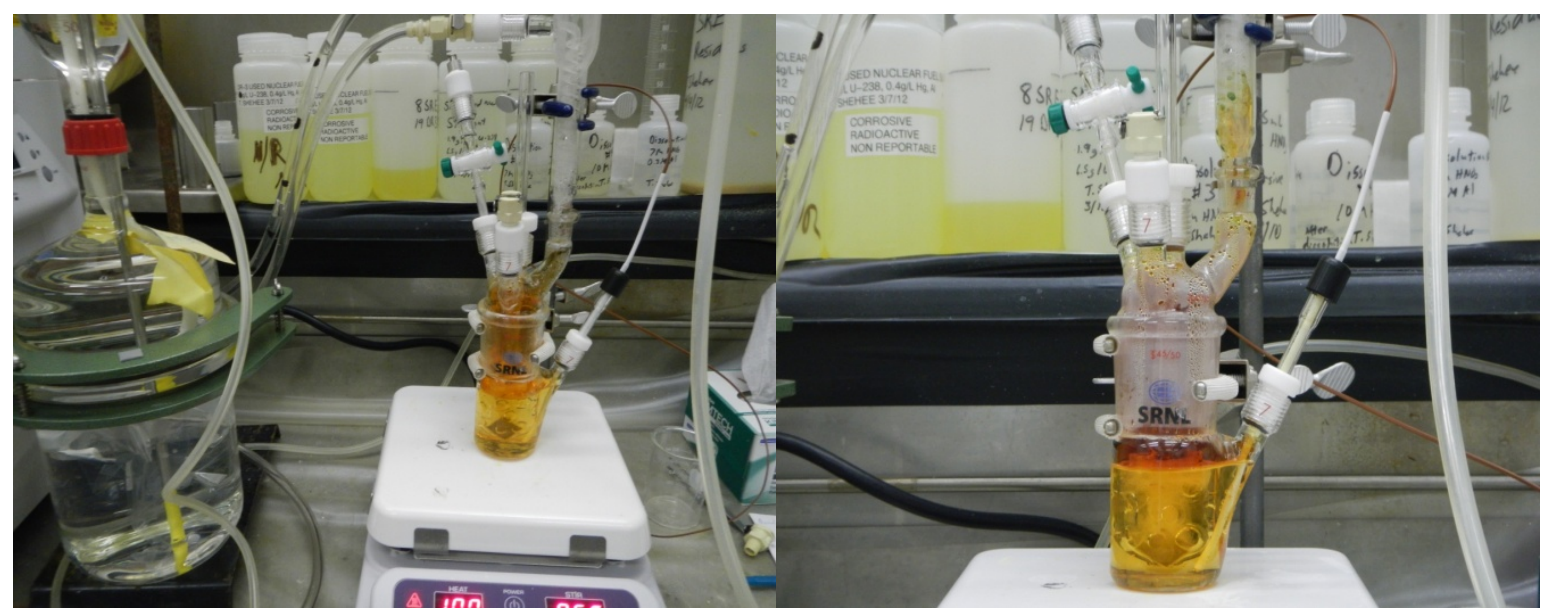

Figure 3-2. Dissolution apparatus showing the dark brown gas generated during the dissolution of an Al coupon.

Table 3-3. Initial results of dissolution studies

\begin{tabular}{|c|c|c|c|c|c|c|c|}
\hline \multirow{2}{*}{$\begin{array}{c}\text { Dissolution } \\
1^{1}\end{array}$} & \multicolumn{2}{|c|}{$\begin{array}{l}\text { Metal foil } \\
\text { used }\end{array}$} & \multirow{2}{*}{$\begin{array}{l}\mathrm{Al}(\mathrm{g}) \\
0.6702\end{array}$} & \multirow{2}{*}{$\begin{array}{c}\text { Th (g) } \\
-\end{array}$} & \multirow{2}{*}{\begin{tabular}{|c|}
$\begin{array}{c}\text { Volume of off- } \\
\text { gas (mL, }\end{array}$ \\
$\sim \mathbf{2 5}{ }^{\circ} \mathbf{C}, \sim 1 \mathbf{~ a t m}, \pm$ \\
$\mathbf{5 m L}$ ) \\
$\begin{array}{c}\text { Exceeded Tedlar } \\
\text { bag volume }\end{array}$
\end{tabular}} & \multirow{2}{*}{$\begin{array}{c}\begin{array}{c}\mathrm{H}_{2} \\
(\mathrm{vol} \% \\
\pm \mathbf{1 0 \%}\end{array} \\
0.55\end{array}$} & \multirow{2}{*}{$\begin{array}{r}\begin{array}{r}\text { Dissolution } \\
\text { time (min) }\end{array} \\
\text { Not measurec }\end{array}$} \\
\hline & - & $\mathrm{Al}$ & & & & & \\
\hline 1-A & - & $\mathrm{Al}$ & 0.6334 & - & 70 & 0.31 & $>125$ \\
\hline 2 & $\mathrm{Th}$ & - & - & 0.8890 & 51.5 & 1.10 & 33 \\
\hline 3 & $\mathrm{Th}$ & - & - & 0.9250 & 60 & 0.18 & 5 \\
\hline 4 & $\mathrm{Th}$ & - & - & 0.9180 & 50 & 0.12 & 3.5 \\
\hline 5 & $\mathrm{Th}$ & - & - & 0.9140 & 85 & 1.7 & 4 \\
\hline $3-A^{3}$ & $\mathrm{Th}^{4+}(\mathrm{aq})$ & $\mathrm{Al}$ & 0.6317 & - & 283 & 0.28 & 4.5 \\
\hline 4- $A^{3}$ & $\mathrm{Th}^{4+}{ }_{(\mathrm{aq})}$ & $\mathrm{Al}$ & 0.6482 & - & 24 & 0.86 & $>25$ \\
\hline $5-A^{3}$ & $\mathrm{Th}^{4+}{ }_{(\mathrm{aq})}$ & $\mathrm{Al}$ & 0.6241 & - & 270 & 1.4 & 6.75 \\
\hline 5-B & $\mathrm{Th}$ & - & & 0.8707 & 75 & 0.5 & 21 \\
\hline 6 & $\mathrm{Th}$ & - & & 1.1011 & 58 & $<0.1$ & 4 \\
\hline 7 & $\mathrm{Th}$ & $\mathrm{Al}$ & 0.9238 & 0.5897 & 126 & 0.9 & 4.5 \\
\hline 8 & $\mathrm{Th}$ & $\mathrm{Al}$ & 1.0327 & 0.5968 & 142 & 0.6 & 5 \\
\hline $9^{4}$ & $\mathrm{Th}$ & $\mathrm{Al}$ & 1.0575 & 0.6517 & 140 & 0.5 & 4.25 \\
\hline $10^{4}$ & $\mathrm{Th}$ & - & & 1.0880 & 77 & 0.4 & 5 \\
\hline \multicolumn{8}{|c|}{ 1) Non-radioactive test of apparatus } \\
\hline \multicolumn{8}{|c|}{ 2) Concentration present after dissolution of FNR and part of SRE bundle } \\
\hline \multicolumn{8}{|c|}{ 3) Dissolver solution is from Th dissolutions 3, 4 and 5. Hg was added to aid in dissolution of Al. } \\
\hline \multicolumn{8}{|c|}{ 4) Duplicate Measurement } \\
\hline
\end{tabular}




\subsection{Off-gas Rate Calculations}

\subsubsection{Off-gas Rate Calculation Assumptions and Conditions}

The prior report ${ }^{34}$ on the dissolution of the MURR assemblies shows that for an immersion depth of 54 inches, an initial $\mathrm{HNO}_{3}$ of $6 \mathrm{M}$, an initial $0.002 \mathrm{M} \mathrm{Hg}$ concentration, an irradiation reduction factor of at least $50 \%$, and a $40 \mathrm{scfm}$ air purge, the peak off-gas rate from the dissolution of four L-Bundles would be $30.9 \mathrm{scfm}$ and the peak off-gas generation for the four MURR assemblies in each of the four L-Bundles would be $14.1 \mathrm{scfm}$. These peak off-gas generation rates from the dissolution of the four L-Bundles and the MURR assemblies are both less than the $34.37 \mathrm{scfm}$ off-gas generation limit to satisfy $60 \% \mathrm{H}_{2} \mathrm{LFL}$ criteria with a $40 \mathrm{scfm}$ air purge.

To calculate the peak off-gas rates for the dissolution of the SRE, HMI, and DR-3 assemblies in the LBundle, several assumptions were made as was done for the dissolution of the MURR assemblies in a prior report ${ }^{34}$. The off-gas rates for the FNR assemblies were not calculated in this report since it was determined in separate documents ${ }^{20,38}$ that the hydrogen off-gas generation from the dissolution of the LBundle with five FNR assemblies would be the same as dissolving the L-Bundle with four MURR assemblies.

First, the chemistry of the dissolution of the Al exterior surfaces for the L-Bundle, SRE, HMI, and DR-3 was assumed to follow the reaction:

$$
\mathrm{Al}_{(\mathrm{s})}+3.75 \mathrm{HNO}_{3} \rightarrow \mathrm{Al}\left(\mathrm{NO}_{3}\right)_{3}+0.225 \mathrm{NO}_{(\mathrm{g})}+0.15 \mathrm{~N}_{2} \mathrm{O}_{(\mathrm{g})}+0.1125 \mathrm{~N}_{2(\mathrm{~g})}+1.875 \mathrm{H}_{2} \mathrm{O}_{(\mathrm{g})}
$$

So 3.75 moles of $\mathrm{HNO}_{3}$ acid are assumed consumed per mole of $\mathrm{Al}$ dissolved. This value was used to estimate how much $\mathrm{HNO}_{3}$ remains after dissolution as well as how much $\mathrm{Al}$ is in solution.

Based on a collection of articles from a symposium of reprocessing irradiated fuels ${ }^{36}$, another potential Th dissolution chemistry reaction is:

$$
\mathrm{Th}_{(\mathrm{s})}+5 \mathrm{HNO}_{3} \rightarrow \mathrm{Th}\left(\mathrm{NO}_{3}\right)_{4}+2.47 \mathrm{H}_{2} \mathrm{O}+0.03 \mathrm{H}_{2(\mathrm{~g})}+0.25 \mathrm{NOx}_{(\mathrm{g})}+0.25 \mathrm{~N}_{2(\mathrm{~g})}
$$

For this reaction 5 moles of $\mathrm{HNO}_{3}$ acid are consumed per mole of thorium (Th) dissolved which matches Karraker's observation. William Prout states in this reference that in practice 5 to 5.2 moles of $\mathrm{HNO}_{3}$ acid are consumed per mole of Th dissolved which matches earlier observations. ${ }^{39}$ Based on these references, it was assumed that 5 moles of $\mathrm{HNO}_{3}$ acid are consumed per mole of Th dissolved.

Since the HMI and DR-3 are High Aluminum-Low Uranium UNFs like the MURR assemblies, the same off-gas composition used for the MURR dissolution calculations ${ }^{34}$ was used. This off-gas composition for the High Aluminum-Low Uranium assemblies is $7.0 \mathrm{vol} \% \mathrm{H}_{2}, 71.4 \mathrm{vol} \% \mathrm{NO}$, and $21.6 \mathrm{vol} \% \mathrm{~N}_{2} \mathrm{O}$ on an air, water, and nitrogen free basis where the molar ratio of $\mathrm{NO} / \mathrm{N}_{2} \mathrm{O}$ is 3.3. Note that this off-gas composition is based on a calculation from $\mathrm{Weitz}^{40}$ on the LFL for H-Canyon Dissolver which points out that the reported gas compositions used to determine the $7 \mathrm{vol} \% \mathrm{H}_{2}$ were actually less than this value (3$5 \mathrm{vol} \% \mathrm{H}_{2}$ ) before placing on a $\mathrm{N}_{2}$ free basis.

The same $\mathrm{H}_{2}$ Lower Flammability Limit (LFL) criteria of $60 \%$ of the $\mathrm{H}_{2}$ LFL value at $200{ }^{\circ} \mathrm{C}$ as was used for the MURR dissolution calculation was used for the HMI, SRE, and DR-3 dissolution calculations. This LFL at $200{ }^{\circ} \mathrm{C}$ was chosen due to the iodine reactor in the off-gas stream of H-Canyon being heated to $200{ }^{\circ} \mathrm{C}$. These $\mathrm{H}_{2} \mathrm{LFL}$ values originally came from F. Scott's $28^{\circ} \mathrm{C}$ data ${ }^{41}$ and are assumed applicable for $\mathrm{NO} / \mathrm{N}_{2} \mathrm{O}$ molar ratios from 2 to 5 . A list of these LFL values is shown in Table 3-4 which came from 
the prior MURR dissolution calculations ${ }^{34}$. Note that the $28{ }^{\circ} \mathrm{C}$ Scott data ${ }^{41}$ was corrected to $200{ }^{\circ} \mathrm{C}$ using a temperature correction from Dyer's work ${ }^{42}$ where:

$$
\mathrm{LFL}_{\mathrm{T}}=\mathrm{LFL}_{\mathrm{ref}} *\left(1-\mathrm{A} *\left(\mathrm{~T}-\mathrm{T}_{\mathrm{ref}}\right)\right)
$$

where:

$\mathrm{LFL}_{\mathrm{T}}=\mathrm{LFL}$ at Temperature $\mathrm{T}\left({ }^{\circ} \mathrm{C}\right)$

$\mathrm{LFL}_{\text {ref }}=\mathrm{LFL}$ at Reference Temperature $\mathrm{T}\left({ }^{\circ} \mathrm{C}\right)$

$\mathrm{A}=$ empirical coefficient (Zabetakis attenuation factor) $=0.0011$

$\mathrm{T}=$ temperature $\left({ }^{\circ} \mathrm{C}\right)$ at which LFL is to be evaluated .

So for the $28{ }^{\circ} \mathrm{C} \mathrm{Scott}$ data the correction to $200{ }^{\circ} \mathrm{C}$ becomes:

$$
\mathrm{LFL}_{200}=\mathrm{LFL}_{28} *(1-0.0011 *(200-28))=\mathrm{LFL}_{28} * 0.811
$$

The $200{ }^{\circ} \mathrm{C} \mathrm{H}_{2} \mathrm{LFL}$ values and $60 \%$ of these values are shown in Table 3-4.

Table 3-4. $\mathbf{H}_{2}$ Lower Flammability Limit from Scott and Dyer Data.

\begin{tabular}{|c|c|c|c|}
\hline Air [vol \%] & Scott $\mathrm{H}_{2}$ LFL at $28{ }^{\circ} \mathrm{C}[\mathrm{vol} \%]$ & Dyer $\mathrm{H}_{2} \mathrm{LFL}$ at $200{ }^{\circ} \mathrm{C}[\mathrm{vol} \%]$ & $60 \%$ of $\mathrm{H}_{2}$ LFL at $200{ }^{\circ} \mathrm{C}[\mathrm{vol} \%]$ \\
\hline 0 & 5.62 & 4.56 & 2.73 \\
\hline 5 & 6.00 & 4.87 & 2.92 \\
\hline 10 & 6.10 & 4.95 & 2.97 \\
\hline 15 & 6.20 & 5.03 & 3.02 \\
\hline 20 & 6.30 & 5.11 & 3.07 \\
\hline 25 & 6.40 & 5.19 & 3.11 \\
\hline 30 & 6.50 & 5.27 & 3.16 \\
\hline 35 & 6.70 & 5.43 & 3.26 \\
\hline 40 & 6.80 & 5.51 & 3.31 \\
\hline 45 & 6.83 & 5.54 & 3.32 \\
\hline 50 & 6.70 & 5.43 & 3.26 \\
\hline 55 & 6.63 & 5.38 & 3.23 \\
\hline 60 & 6.50 & 5.27 & 3.16 \\
\hline 65 & 6.20 & 5.03 & 3.02 \\
\hline 70 & 5.70 & 4.62 & 2.77 \\
\hline 75 & 5.60 & 4.54 & 2.72 \\
\hline 80 & 5.20 & 4.22 & 2.53 \\
\hline 85 & 4.60 & 3.73 & 2.24 \\
\hline 90 & 4.30 & 3.49 & 2.09 \\
\hline 96 & 4.00 & 3.24 & 1.95 \\
\hline
\end{tabular}

In the prior MURR dissolution off-gas calculations ${ }^{34}$, the maximum peak off-gas rate of $34.37 \mathrm{scfm}$ during dissolution was calculated based on the assumed off-gas composition discussed above with a 40 scfm air purge rate to stay below the $60 \%$ of $\mathrm{H}_{2} \mathrm{LFL}$ at $200{ }^{\circ} \mathrm{C}$. This same peak off-gas rate of 34.37 scfm will be used for the HMI, SRE, and DR-3 dissolutions. The reason the same flammability limit was applied to the thorium SRE assembly is that the thorium fuel is housed in an aluminum bundle tube which sits in the L-Bundle as shown in Appendix A.2. The exposed surface area of the thorium fuel inside the SRE aluminum bundle tube is about an order of magnitude smaller than the outer area of the SRE aluminum bundle tube and the aluminum L-Bundle. Using the flammability limits from the MURR 
dissolution is also conservative since the off-gas composition data for dissolving pure metal thorium is less than $7 \mathrm{vol} \% \mathrm{H}_{2}$. The literature and experimental off-gas composition values for dissolving thorium metal only are shown in Table 3-5. The highest vol $\% \mathrm{H}_{2}$ value from the literature and from the current experiments discussed earlier is $3.6 \mathrm{vol} \%$ on a water free basis. Since the thorium dissolution off-gas 3.6 vol $\% \mathrm{H}_{2}$ is smaller than the $\mathrm{Al}$ dissolution off-gas $7 \mathrm{vol} \% \mathrm{H}_{2}$ and the thorium surface area is smaller than the aluminum surface area, using the Al dissolution off-gas LFL's is conservative.

Table 3-5. Off-gas Composition Values for Dissolving Thorium Metal in Solution without Th Present Initially

\begin{tabular}{|c|c|c|c|c|c|c|c|c|c|c|c|}
\hline Data Source & $\begin{array}{c}\text { Initial } \\
\mathrm{HNO}_{3} \\
{[\mathrm{M}]}\end{array}$ & $\begin{array}{l}\text { Initial } \\
\mathrm{F}[\mathrm{M}]\end{array}$ & $\begin{array}{c}\text { Initial } \\
\text { Th }[\mathrm{M}]\end{array}$ & $\begin{array}{l}\text { Initial } \\
\mathrm{Al}[\mathrm{M}]\end{array}$ & {$\left[\begin{array}{c}\mathrm{H}_{2} \\
{[\mathrm{vol} \%]}\end{array}\right]$} & $\left.\mid \begin{array}{c}\mathrm{NO} \\
{[\mathrm{vol} \%]}\end{array}\right]$ & $\begin{array}{c}\mathrm{N}_{2} \\
\text { [vol \%] }\end{array}$ & $\begin{array}{c}\mathrm{NO}_{2} \\
{[\mathrm{vol} \%]}\end{array}$ & $\begin{array}{c}\mathrm{N}_{2} \mathrm{O} \\
{[\mathrm{vol} \%]}\end{array}$ & $\left.\mid \begin{array}{c}\mathrm{O}_{2} \\
{[\mathrm{vol} \%]}\end{array}\right]$ & $\begin{array}{c}\mathrm{H}_{2} \mathrm{O} \\
{[\mathrm{vol} \%]}\end{array}$ \\
\hline $\begin{array}{l}\text { Karraker }^{35} \\
\text { Water Free }\end{array}$ & 10 & $\begin{array}{c}0.05- \\
0.1\end{array}$ & 0 & 0 & 3.6 & 86.8 & 7.8 & 0.6 & 0.6 & 0.6 & 0 \\
\hline Symposium $^{36}$ & 10 & 0.05 & 0 & 0 & 2.5 & 9 & 13 & 73 & 2.5 & 0 & 0 \\
\hline Dissolution 4 & 10 & 0.05 & 0 & 0 & 0.1 & 0 & 99.6 & 0 & 0 & 0.3 & 0 \\
\hline Dissolution 6 & 10 & 0.05 & 0 & 0.3 & $<0.1$ & $<0.1$ & 27.5 & 0 & $<0.1$ & 7.9 & 0 \\
\hline Dissolution 5-B & 7 & 0.01 & 0 & 0.3 & 0.5 & $<0.1$ & 5.8 & 0 & $<0.1$ & 1.0 & 0 \\
\hline Dissolution 3 & 7 & 0.05 & 0 & 0 & 0.2 & 0 & 99.7 & 0 & 0 & $<0.1$ & 0 \\
\hline Dissolution 5 & 7 & 0.05 & 0 & 0.3 & 1.7 & 0 & 98.2 & 0 & 0 & $<0.1$ & 0 \\
\hline Dissolution 10 & 7 & 0.05 & 0 & 0.3 & 0.4 & $<0.1$ & 9.7 & 0 & 0.9 & 0.8 & 0 \\
\hline Dissolution 2 & 4 & 0.05 & 0 & 0 & 1.1 & 0 & 87.8 & 0 & 0 & 11.1 & 0 \\
\hline
\end{tabular}

Note: Dissolutions 1-5 in Table 2-1 used $\mathrm{N}_{2}$ as carrier gas and the rest used argon as the carrier gas.

Dissolution 2 was contaminated with air during analytical handling.

In the off-gas calculations for the dissolution of the MURR assemblies ${ }^{34}$, the reference peak off-gas generation rate was $0.676 \mathrm{scfm} / \mathrm{ft}^{2}$ at $0.001 \mathrm{M} \mathrm{Hg}$ based on testing by Caracciolo. ${ }^{43}$ This same reference peak off-gas rate was assumed for the dissolution of the SRE, HMI, and DR-3 assemblies in the LBundle. Note that this High Aluminum-Low Uranium off-gas generation rate can be used for the thorium SRE assembly since the thorium SRE fuel is housed in an aluminum bundle tube which sits in the Disassembly L Area Bundling Tube (DABT) or L-Bundle. From current experiments discussed earlier, the measured off-gas generation rate for dissolving pure thorium metal is 0.004 to $0.055 \mathrm{scfm} / \mathrm{ft}^{2}$ as shown in Table 3-6. After the L-Bundle is dissolved followed by the outer aluminum bundling tube of the SRE then the SRE fuel tubes will dissolve but based on the expected off-gas generation rate and the lower surface area, the prior off-gas generation rates for the exterior portions of the SRE will be bounding.

Table 3-6. Peak Off-gas Generation Rate for Thorium Dissolving Thorium Metal

\begin{tabular}{|c|c|c|c|c|c|}
\hline Data Source & $\begin{array}{c}\text { Initial } \mathrm{HNO}_{3} \\
{[\mathrm{M}]}\end{array}$ & Initial F [M] & Initial Th $[\mathrm{M}]$ & Initial Al [M] & Off-gas Generation [scfm/ $\left.\mathrm{ft}^{2}\right]$ \\
\hline Dissolution 4 & 10 & 0.05 & 0 & 0 & 0.037 \\
\hline Dissolution 6 & 10 & 0.05 & 0 & 0.3 & 0.034 \\
\hline Dissolution 5-B & 7 & 0.01 & 0 & 0.3 & 0.010 \\
\hline Dissolution 3 & 7 & 0.05 & 0 & 0 & 0.030 \\
\hline Dissolution 5 & 7 & 0.05 & 0 & 0.3 & 0.055 \\
\hline Dissolution 10 & 7 & 0.05 & 0 & 0.3 & 0.039 \\
\hline Dissolution 2 & 4 & 0.05 & 0 & 0 & 0.004 \\
\hline
\end{tabular}

Additional experimental details are found in Table 2-1 
Based on work by Robert Moore ${ }^{44}$ the thorium dissolution rate is linearly proportional to the $\mathrm{F}$ concentration, which acts like a catalyst. For the planned dissolution of the SRE assemblies, the initial HF concentration was assumed to be $0.05 \mathrm{M}$. In the Moore reference, it states that the thorium dissolution rate is second order with respect to the initial $\mathrm{HNO}_{3}$ concentration which means that the initial $\mathrm{HNO}_{3}$ concentration has a bigger effect on the dissolution rate than the $\mathrm{F}$ concentration.

The $\mathrm{Al}$ dissolution rate is assumed linearly proportional to the $\mathrm{Hg}$ concentration as was done in the MURR dissolution calculations ${ }^{34}$. For the planned dissolution of the HMI, SRE, and DR-3 assemblies the initial $\mathrm{Hg}$ concentration was assumed to be $0.002 \mathrm{M}$.

The dissolution rate of the MURR assemblies was affected by the irradiation of the fuel assemblies by a $50 \%$ reduction due to hardening of surfaces as described in the prior dissolution calculations. ${ }^{34}$ This $50 \%$ reduction in dissolution rate directly translates into a 50\% reduction of the peak off-gas generation rate. Since the HMI and DR-3 assemblies are comparable to the MURR assemblies (High Aluminum-low Uranium), a similar 50\% reduction in dissolution rate was assumed for these off-gas calculations. However, for the SRE assemblies the $\mathrm{U}^{235}$ burn-up is about $23 \%$ versus the about $54-65 \% \mathrm{U}^{235}$ burn-up of the HMI and DR-3 fuels and so no reduction in dissolution rate for the SRE assemblies was assumed. This zero impact of irradiation on the dissolution of the thorium fuels is conservative or will indicate higher off-gas rates than actually expected.

For the prior dissolution calculation of the MURR assemblies ${ }^{34}$ the impact of dissolved Al on the dissolution rate was characterized using Equation 53:

$$
\mathrm{r}_{\mathrm{Al}}=102.64 * 10^{-1.078 *[\mathrm{Al}]}
$$

where:

$$
\begin{aligned}
& \mathrm{r}_{\mathrm{Al}}=\mathrm{Al} \text { dissolution rate in } \mathrm{mg} / \mathrm{cm}^{2} / \mathrm{min} \\
& {[\mathrm{Al}]=\text { molar concentration of dissolved } \mathrm{Al}}
\end{aligned}
$$

In the prior dissolution calculations for the MURR assemblies this correlation was used to scale down the peak dissolution rate of $52.53 \mathrm{mg} / \mathrm{cm}^{2} / \mathrm{min}$ at about $0.27 \mathrm{M} \mathrm{Al}$ when calculating the impact of the dissolved $\mathrm{Al}$ from a prior dissolution batch. This adjustment to the dissolution rate based on dissolved Al can be expressed as:

$$
r_{a d j}=\frac{102.64 * 10^{-1.078 *[A l]}}{52.53}
$$

where:

$\mathrm{r}_{\mathrm{adj}}=\mathrm{Al}$ dissolution rate adjustment factor for dissolved $\mathrm{Al}$

$[\mathrm{Al}]=$ molar concentration of dissolved $\mathrm{Al}$

A compilation of the thorium dissolution rate data from literature ${ }^{44,36}$ and from the current experiments is shown in Table 3-7. A plot of the thorium dissolution rate as a function of the $\mathrm{HNO}_{3}$ and $\mathrm{Al}$ concentrations is shown in Figure 3-3. This plot shows that at high $\mathrm{HNO}_{3}$ concentrations near $13 \mathrm{M}$ some dissolved $\mathrm{Al}$ near $0.5 \mathrm{M}$ has a significant impact on the thorium dissolution rate. This plot also shows that at lower $\mathrm{HNO}_{3}$ concentrations near $7 \mathrm{M}$ the impact of dissolved $\mathrm{Al}$ is small and negligible when considering measurement uncertainty. For these calculations it was assumed that the SRE dissolution will take place at an initial $\mathrm{HNO}_{3}$ concentration less than or equal to $7 \mathrm{M}$ and the impact of dissolved $\mathrm{Al}$ on the thorium dissolution rate is negligible. 
Examining only the thorium dissolution rates in Table 3-7 with no initial dissolved Al, the thorium dissolution rate is second order with respect to the $\mathrm{HNO}_{3}$ concentration as indicated in the literature. For this set of the data a second order fit of the thorium dissolution rates with no initial dissolved $\mathrm{Al}$ is shown to be:

$$
\mathrm{r}_{\mathrm{Th}}=2.7074 *\left[\mathrm{HNO}_{3}\right]^{2}-32.416 *\left[\mathrm{HNO}_{3}\right]+91.425
$$

where:

$\mathrm{r}_{\mathrm{Th}}=$ Th Dissolution Rate in $\mathrm{mg} / \mathrm{cm}^{2} / \mathrm{min}$

$\left[\mathrm{HNO}_{3}\right]=$ Molar Concentration of $\mathrm{HNO}_{3}$

The thorium dissolution rates with no dissolved Al in Table 3-7 from the Symposium on the Reprocessing of Irradiated Fuels document ${ }^{36}$ were interpolated from a figure on Slide 6.2 of that reference. These thorium dissolution rates were then fitted with cubic functions of the $\mathrm{HNO}_{3}$ and thorium concentrations as shown in Figure 3-4. Table 3-8 shows the various cubic fits for the thorium dissolution rates. Note the $\mathrm{R}^{2}$ correlation factors of these cubic fits were high ( 0.99 or greater) so these fits are a good approximation to the original figure in Slide 6.2 of the Symposium document.

Using these cubic fits, the thorium dissolution rates at $7 \mathrm{M}$ and $10 \mathrm{M} \mathrm{HNO}_{3}$ concentration with $0.05 \mathrm{M} \mathrm{F}$ and no initial Al were calculated as shown in Table 3-9. A plot of these predicted thorium dissolution rates is shown in Figure 3-5. These cubic fits can be used for any off-gas calculations for thorium metal dissolution at or below $7 \mathrm{M} \mathrm{HNO}_{3}$ ignoring the impact of the dissolved Al.

Table 3-7. Thorium Dissolution Rate Data

\begin{tabular}{|c|c|c|c|c|c|}
\hline Data Source & $\begin{array}{c}\text { Initial } \\
\mathrm{HNO}_{3}[\mathrm{M}]\end{array}$ & $\begin{array}{c}\text { Initial Al } \\
{[\mathrm{M}]}\end{array}$ & Initial $\mathrm{F}[\mathrm{M}]$ & Initial Th $[\mathrm{M}]$ & $\begin{array}{l}\text { Th Dissolution } \\
{\left[\mathrm{mg} / \mathrm{cm}^{2} / \mathrm{min}\right]}\end{array}$ \\
\hline Moore & 7 & 0.5 & 0.05 & 0 & 14.83 \\
\hline Moore & 13 & 0.5 & 0.05 & 0 & 4.17 \\
\hline Moore & 13 & 0 & 0.05 & 0.2 & 94.83 \\
\hline Moore & 13 & 0 & 0.05 & 0.5 & 66.83 \\
\hline Moore & 13 & 0 & 0.05 & 0.72 & 49.17 \\
\hline Moore & 13 & 0 & 0.05 & 1 & 29.83 \\
\hline Moore & 7 & 0 & 0.05 & 0.5 & 23.17 \\
\hline Moore & 13 & 0 & 0.05 & 0 & 133.33 \\
\hline Dissolution 2** & 4 & 0 & 0.05 & 0 & 2.19 \\
\hline Dissolution $3 * *$ & 7 & 0 & 0.05 & 0 & 14.48 \\
\hline Dissolution $4 * *$ & 10 & 0 & 0.05 & 0 & 20.69 \\
\hline Dissolution $5^{* *}$ & 7 & 0.3 & 0.05 & 0 & 18.10 \\
\hline Dissolution 5-B** & 7 & 0.3 & 0.01 & 0 & 3.49 \\
\hline Dissolution $6^{* *}$ & 10 & 0.3 & 0.05 & 0 & 19.65 \\
\hline Dissolution $10^{* *}$ & 7 & 0.3 & 0.05 & 0 & 17.03 \\
\hline Symposium* & 2 & 0 & 0.05 & 0 & 21 \\
\hline Symposium* & 4 & 0 & 0.05 & 0 & 38 \\
\hline Symposium* & 6 & 0 & 0.05 & 0 & 58 \\
\hline Symposium* & 8 & 0 & 0.05 & 0 & 84 \\
\hline Symposium* & 10 & 0 & 0.05 & 0 & 111 \\
\hline Symposium* & 12 & 0 & 0.05 & 0 & 130 \\
\hline Symposium* & 14 & 0 & 0.05 & 0 & 134 \\
\hline
\end{tabular}


Table 3-7. Thorium Dissolution Rate Data

\begin{tabular}{|c|c|c|c|c|c|}
\hline Data Source & $\begin{array}{c}\text { Initial } \\
\mathrm{HNO}_{3}[\mathrm{M}]\end{array}$ & $\begin{array}{c}\text { Initial Al } \\
{[\mathrm{M}]}\end{array}$ & Initial $\mathrm{F}[\mathrm{M}]$ & Initial Th $[\mathrm{M}]$ & $\begin{array}{l}\text { Th Dissolution } \\
{\left[\mathrm{mg} / \mathrm{cm}^{2} / \mathrm{min}\right]}\end{array}$ \\
\hline Symposium* & 2 & 0 & 0.05 & 0.2 & 11 \\
\hline Symposium* & 4 & 0 & 0.05 & 0.2 & 21 \\
\hline Symposium* & 6 & 0 & 0.05 & 0.2 & 35 \\
\hline Symposium* & 8 & 0 & 0.05 & 0.2 & 54 \\
\hline Symposium* & 10 & 0 & 0.05 & 0.2 & 77 \\
\hline Symposium* & 12 & 0 & 0.05 & 0.2 & 93 \\
\hline Symposium* & 14 & 0 & 0.05 & 0.2 & 95 \\
\hline Symposium* & 2 & 0 & 0.05 & 0.5 & 4 \\
\hline Symposium* & 4 & 0 & 0.05 & 0.5 & 10 \\
\hline Symposium* & 6 & 0 & 0.05 & 0.5 & 18 \\
\hline Symposium* & 8 & 0 & 0.05 & 0.5 & 32 \\
\hline Symposium* & 10 & 0 & 0.05 & 0.5 & 57 \\
\hline Symposium* & 12 & 0 & 0.05 & 0.5 & 67 \\
\hline Symposium* & 14 & 0 & 0.05 & 0.5 & 68 \\
\hline Symposium* & 2 & 0 & 0.05 & 0.72 & 2 \\
\hline Symposium* & 4 & 0 & 0.05 & 0.72 & 7 \\
\hline Symposium* & 6 & 0 & 0.05 & 0.72 & 16 \\
\hline Symposium* & 8 & 0 & 0.05 & 0.72 & 28 \\
\hline Symposium* & 10 & 0 & 0.05 & 0.72 & 47 \\
\hline Symposium* & 12 & 0 & 0.05 & 0.72 & 51 \\
\hline Symposium* & 14 & 0 & 0.05 & 0.72 & 49 \\
\hline Symposium* & 2 & 0 & 0.05 & 1 & 1 \\
\hline Symposium* & 4 & 0 & 0.05 & 1 & 5 \\
\hline Symposium* & 6 & 0 & 0.05 & 1 & 12 \\
\hline Symposium* & 8 & 0 & 0.05 & 1 & 22 \\
\hline Symposium* & 10 & 0 & 0.05 & 1 & 28 \\
\hline Symposium* & 12 & 0 & 0.05 & 1 & 30 \\
\hline Symposium* & 14 & 0 & 0.05 & 1 & 30 \\
\hline
\end{tabular}

*Interpolated value from figure on Slide 6.2 from Symposium Reference, **Experiments in Table 2-1 
SRNL-STI-2012-00279

Revision 1

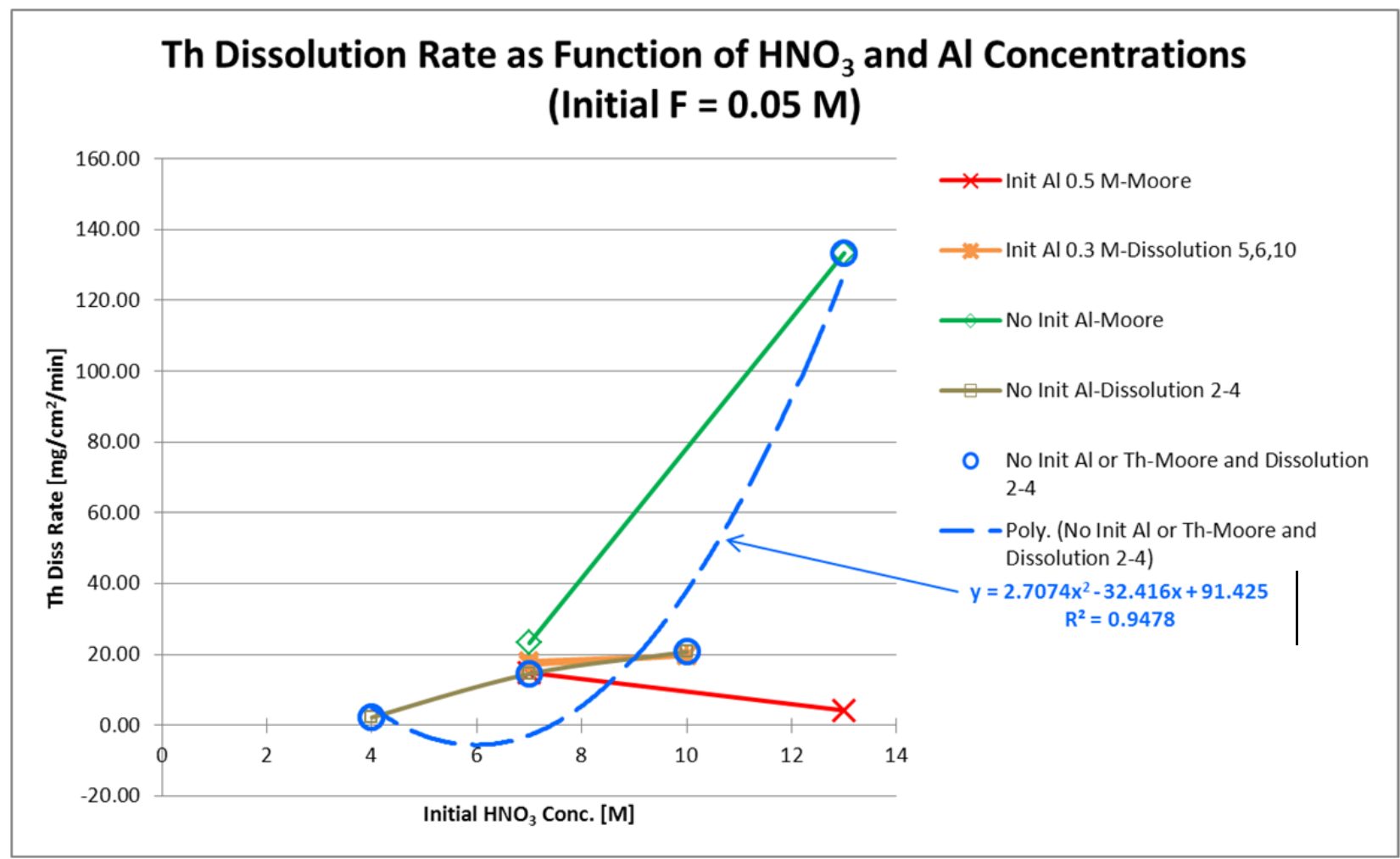

Figure 3-3. Th Dissolution Rate as function of $\mathrm{HNO}_{3}$ and Dissolved Al

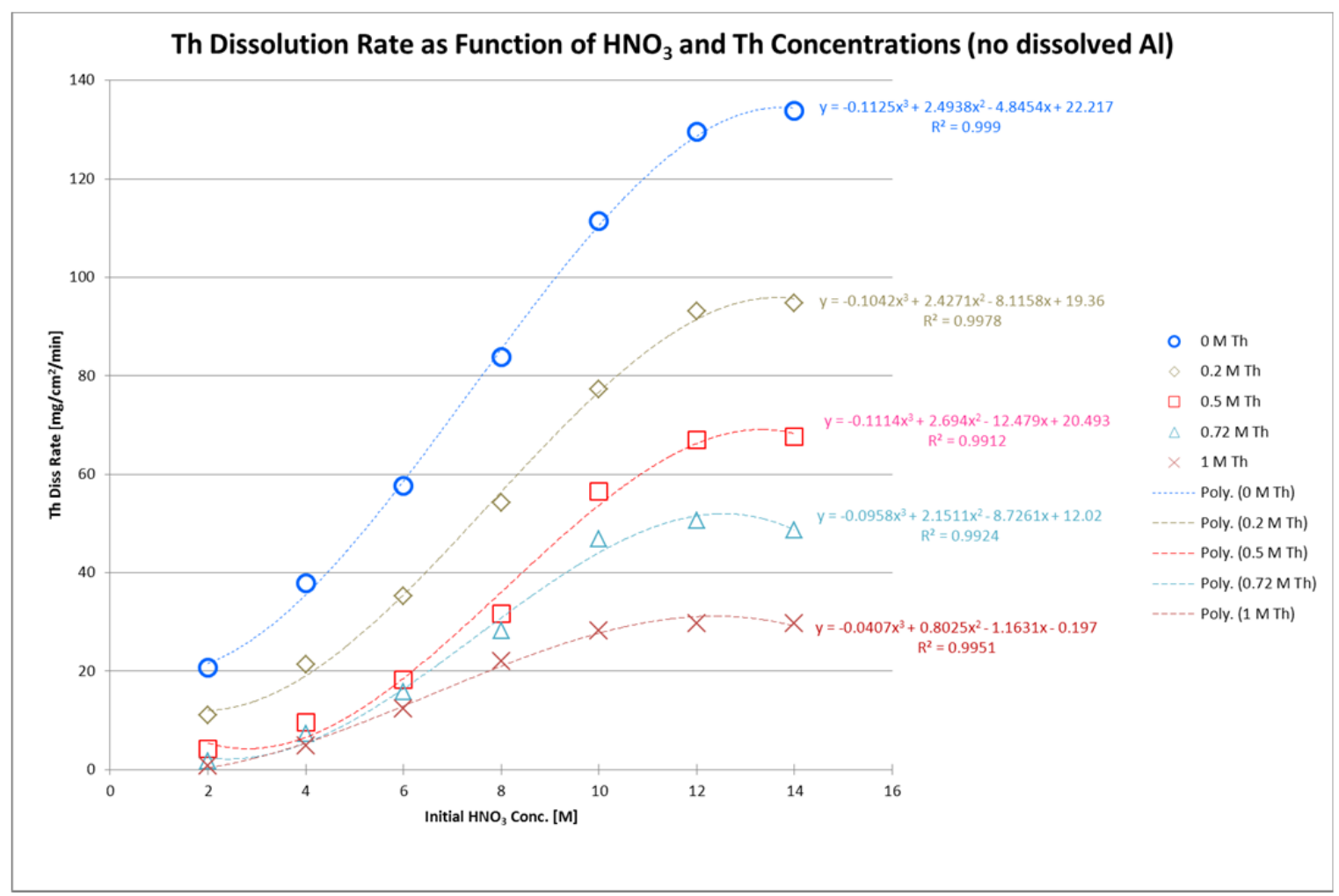

Figure 3-4. Th Dissolution Rate as function of $\mathrm{HNO}_{3}$ and Th Concentrations 
Table 3-8. Thorium Dissolution Rate Fits as function of $\mathrm{HNO}_{3}$ and thorium Concentrations

\begin{tabular}{|c|c|c|c|c|c|}
\hline $\begin{array}{c}\text { Initial } \\
\mathrm{HNO}_{3}[\mathrm{M}]\end{array}$ & $\begin{array}{c}\text { Initial } \\
\mathrm{F}[\mathrm{M}]\end{array}$ & $\begin{array}{c}\text { Initial } \\
\text { Th }[\mathrm{M}]\end{array}$ & $\begin{array}{c}\text { Initial } \\
\mathrm{Al}[\mathrm{M}]\end{array}$ & Th Dissolution Rate $\left[\mathrm{mg} / \mathrm{cm}^{2} / \mathrm{min}\right]$ & $\begin{array}{c}\mathrm{R}^{2} \text { Corre- } \\
\text { lation } \\
\text { Factor }\end{array}$ \\
\hline $2-14$ & 0.05 & 0 & 0 & $\mathrm{r}=-0.1125^{*}\left[\mathrm{HNO}_{3}\right]^{3}+2.4938 *\left[\mathrm{HNO}_{3}\right]^{2}-4.8454 *\left[\mathrm{HNO}_{3}\right]+22.217$ & 0.999 \\
\hline $2-14$ & 0.05 & 0.2 & 0 & $\mathrm{r}=-0.1042\left[\mathrm{HNO}_{3}\right]^{3}+2.4271\left[\mathrm{HNO}_{3}\right]^{2}-8.1158\left[\mathrm{HNO}_{3}\right]+19.36$ & 0.998 \\
\hline $2-14$ & 0.05 & 0.5 & 0 & $\mathrm{r}=-0.1114\left[\mathrm{HNO}_{3}\right]^{3}+2.694\left[\mathrm{HNO}_{3}\right]^{2}-12.479\left[\mathrm{HNO}_{3}\right]+20.493$ & 0.991 \\
\hline $2-14$ & 0.05 & 0.72 & 0 & $\mathrm{r}=-0.0958\left[\mathrm{HNO}_{3}\right]^{3}+2.1511\left[\mathrm{HNO}_{3}\right]^{2}-8.7261\left[\mathrm{HNO}_{3}\right]+12.02$ & 0.992 \\
\hline $2-14$ & 0.05 & 1 & 0 & $\mathrm{r}=-0.0407\left[\mathrm{HNO}_{3}\right]^{3}+0.8025\left[\mathrm{HNO}_{3}\right]^{2}-1.1631\left[\mathrm{HNO}_{3}\right]-0.197$ & 0.995 \\
\hline
\end{tabular}

Table 3-9. Predicted Thorium Dissolution Rates at 7 and $10 \mathrm{M} \mathrm{HNO}_{3}$ and $0.05 \mathrm{M} \mathrm{F}$

\begin{tabular}{|c|c|c|c|c|}
\hline $\begin{array}{c}\text { Initial } \\
\mathrm{HNO}_{3}[\mathrm{M}]\end{array}$ & $\begin{array}{c}\text { Initial } \\
\mathrm{F}[\mathrm{M}]\end{array}$ & $\begin{array}{c}\text { Initial } \\
\text { Th }[\mathrm{M}]\end{array}$ & $\begin{array}{c}\text { Initial } \\
\mathrm{Al}[\mathrm{M}]\end{array}$ & $\begin{array}{c}\text { Th Dissolution Rate } \\
{\left[\mathrm{mg} / \mathrm{cm}^{2} / \mathrm{min}\right]}\end{array}$ \\
\hline 7 & 0.05 & 0 & 0 & 72 \\
\hline 7 & 0.05 & 0.2 & 0 & 46 \\
\hline 7 & 0.05 & 0.5 & 0 & 27 \\
\hline 7 & 0.05 & 0.72 & 0 & 23 \\
\hline 7 & 0.05 & 1 & 0 & 17 \\
\hline 10 & 0.05 & 0 & 0 & 111 \\
\hline 10 & 0.05 & 0.2 & 0 & 77 \\
\hline 10 & 0.05 & 0.5 & 0 & 54 \\
\hline 10 & 0.05 & 0.72 & 0 & 44 \\
\hline 10 & 0.05 & 1 & 0 & 28 \\
\hline
\end{tabular}

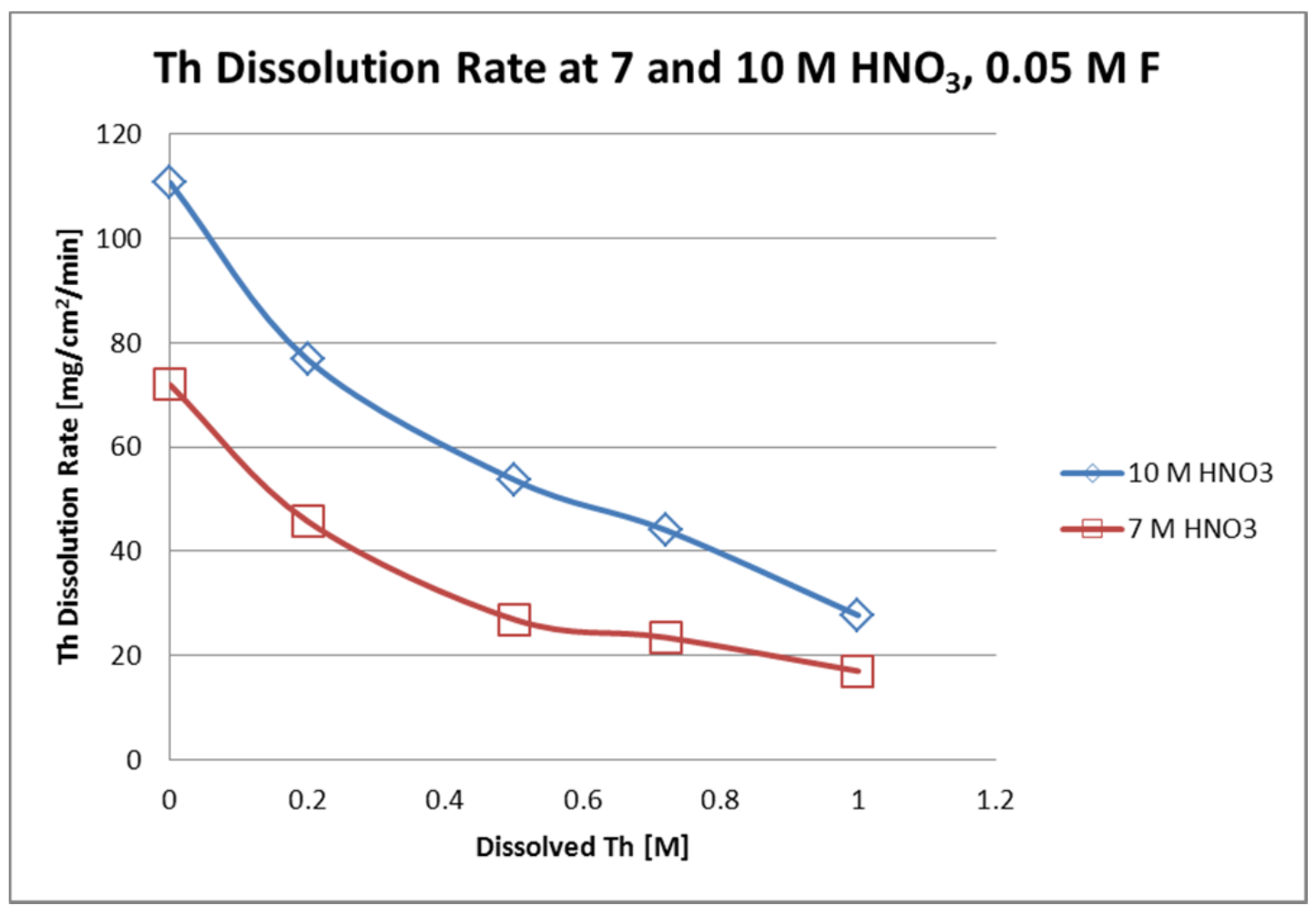

Figure 3-5. Th Dissolution Rate at 7 and 10 M HNO3, 0.05 M F as Function of Th Concentration 
SRNL-STI-2012-00279

Revision 1

\subsubsection{Off-gas Rate Values for Dissolver Charging Scenarios}

Using the off-gas rate calculation assumptions and conditions defined in the prior section, the peak offgas rates were determined to be bounded by the controls listed in the off-gas calculations for the MURR assemblies. $^{34}$ Initially, up to 4 bundles of FNR, SRE, HMI, and/or DR-3 can be charged to a solution of fresh nitric acid. After the first dissolution, the number of bundles that can be charged is dependent on the concentration of aluminum in the dissolver solution as shown in Table 8 and Figure 8 of the off-gas calculations for the MURR assemblies ${ }^{34}$.

An example of the off-gas calculations for the initial charge of 4 bundles of SRE is shown in Table 3-10 using the dissolution terms defined in the prior section which originate from the off-gas calculations for the MURR assemblies. ${ }^{34}$ The peak off-gas rate for processing the 4 L-Bundles of SRE assemblies is $34.80 \mathrm{scfm}$ which slightly exceeds the $34.37 \mathrm{scfm} 60 \% \mathrm{H}_{2} \mathrm{LFL}$ at $200{ }^{\circ} \mathrm{C}$ or represents $3.26 \% \mathrm{H}_{2}$ instead of the limit of $3.2 \% \mathrm{H}_{2}$. Since the assumption of $7 \mathrm{vol} \% \mathrm{H}_{2}$ in the off-gas is conservative as discussed earlier or the actual off-gas values were less than the base average 7 vol $\%$ and no credit is taken for the reduction in rate due to irradiation of the SRE assemblies $(23 \%)$, the $34.80 \mathrm{scfm}$ peak off-gas is acceptable. After the 4 outer L-Bundles dissolve, the peak off-gas rate for processing the 4 outer bundling tubes of the SRE assemblies themselves is $22.54 \mathrm{scfm}$ labeled as Peak Off-Gas for Assembly Area 1 in Table 3-10. If there is a possibility of a hole or cut in any of the 4 outer bundling tubes of the SRE assemblies inside the 4 L-Bundles then the off-gas rate based on the inside exposed fuel area of the SRE assemblies assuming it reacts like Al would be $2.82 \mathrm{scfm}$ labeled as Peak Off-gas for Assembly Area 2. If one uses the thorium dissolution off-gas rates discussed earlier then the peak off-gas rates for the exposed inside fuel tubes will be much smaller. This example calculation demonstrates that the dissolving of the outer L-Bundle will generally dictate how many bundles are processed per dissolver charging scenario as shown in the prior off-gas calculations for the MURR assemblies. ${ }^{34}$ 
Table 3-10. Example Off-Gas Rates for Dissolver Batching Scenario with 4 SRE L-Bundles

\begin{tabular}{|c|c|c|c|c|c|c|c|c|c|c|c|c|c|c|}
\hline $\begin{array}{l}\text { Dissolver } \\
\text { Batches }\end{array}$ & $\begin{array}{l}\text { Fuel } \\
\text { Type }\end{array}$ & $\begin{array}{c}\text { L- } \\
\text { Bundles }\end{array}$ & $\begin{array}{l}\text { Assemblies } \\
\text { per Bundle }\end{array}$ & $\begin{array}{c}\text { Initial } \\
\mathrm{HNO}_{3} \\
{[\mathrm{M}]}\end{array}$ & $\begin{array}{c}\text { Final } \\
\mathrm{HNO}_{3} \\
{[\mathrm{M}]}\end{array}$ & $\begin{array}{l}\text { Initial Al } \\
\text { Conc }[\mathbf{M}]\end{array}$ & $\begin{array}{c}\text { Final Al } \\
\text { Conc } \\
{[\mathbf{M}]}\end{array}$ & $\mid \begin{array}{l}\text { Initial Th } \\
\text { Conc }[\mathrm{M}]\end{array}$ & $\begin{array}{c}\text { Final Th } \\
\text { Conc } \\
\text { [M] }\end{array}$ & \begin{tabular}{|c} 
Moles of \\
HNO3 \\
consumed \\
per mole \\
of Al \\
dissolved
\end{tabular} & $\begin{array}{c}\text { Moles of } \\
\text { HNO3 } \\
\text { consumed } \\
\text { per mole of } \\
\text { Th } \\
\text { dissolved }\end{array}$ & $\begin{array}{c}\text { Hg [M] } \\
\text { (assume } \\
\text { constant) }\end{array}$ & $\begin{array}{c}\text { F [M] } \\
\text { (assume } \\
\text { constant) }\end{array}$ & $\begin{array}{c}\text { Exposed } \\
\text { Metal }\end{array}$ \\
\hline 1 & SRE & 4 & 1 & 2.40 & 1.23 & 0.00 & 0.22 & 0.00 & 0.07 & 3.75 & 5.00 & 0.0020 & 0.05 & $\mathrm{Al}$ \\
\hline
\end{tabular}

\begin{tabular}{|c|c|c|c|c|c|c|c|c|c|c|c|c|c|}
\hline $\begin{array}{c}\text { Ref. Peak } \\
\text { Off-gas } \\
\text { Rate } \\
{\left[\mathbf{s c f m} / \mathbf{f t}^{2}\right]}\end{array}$ & $\mid \begin{array}{c}\text { Ref. Peak } \\
\text { Off-gas } \\
\text { Rate Hg } \\
\text { conc. }[\mathrm{M}]\end{array}$ & $\begin{array}{c}\text { Scale for } \\
\text { Ref. Peak } \\
\text { Off-gas } \\
\text { Rate } \\
\text { based on } \\
\text { Diss Al }\end{array}$ & \begin{tabular}{|c} 
Scale for \\
Ref. Peak \\
Off-gas \\
Rate based \\
on \\
Irradiation
\end{tabular} & $\begin{array}{c}\text { Scale for } \\
\text { Ref. Peak } \\
\text { Off-gas } \\
\text { Rate based } \\
\text { on Catalyst } \\
\text { Conc }\end{array}$ & $\begin{array}{c}\text { Corrected } \\
\text { Peak Off- } \\
\text { gas Rate } \\
{\left[\mathrm{scfm} / \mathrm{ft}^{2}\right]}\end{array}$ & $\begin{array}{l}\text { Immersion } \\
\text { Depth [in] }\end{array}$ & $\begin{array}{c}\text { Immersio } \\
\text { n Depth } \\
\text { [ft] }\end{array}$ & $\begin{array}{c}\text { Bundle } \\
\text { Outer } \\
\text { Surface } \\
\text { Area }\left(\mathbf{f t}^{2}\right)\end{array}$ & $\begin{array}{c}\text { Peak Off- } \\
\text { gas for } \\
\text { Bundle } \\
\text { Outer Area } \\
\text { [scfm] }\end{array}$ & $\begin{array}{c}\text { Assembly } \\
\text { Surface } \\
\text { Area 1 } \\
{\left[\text { in }^{2}\right]}\end{array}$ & $\begin{array}{l}\text { Peak Off- } \\
\text { gas for } \\
\text { Assembly } \\
\text { Area 1 } \\
\text { [scfm] }\end{array}$ & $\begin{array}{c}\text { Assembly } \\
\text { Surface } \\
\text { Area } 2 \\
\left.\text { [in }^{2}\right]\end{array}$ & $\begin{array}{l}\text { Peak Off- } \\
\text { gas for } \\
\text { Assembly } \\
\text { Area } 2 \\
\text { [scfm] }\end{array}$ \\
\hline 0.676 & 0.001 & 1 & 1 & 1.99 & 1.35 & 54 & 4.5 & 25.8 & 34.80 & 16.7 & 22.54 & 2.1 & 2.82 \\
\hline
\end{tabular}




\subsection{Fuel Simulant Neutralization}

Neutralization experiments using $50 \mathrm{wt} \%$ sodium hydroxide targeted 4 neutralization points. The acidic solution was neutralized to the $\mathrm{pH}$ where the first solids precipitate and do not redissolve, $\mathrm{pH}$ of $\sim 4.5$; to $\mathrm{pH} 7$; to $0.8 \mathrm{M}$ free hydroxide, and to $1.2 \mathrm{M}$ free hydroxide. Figure 3-6 below shows the samples at several of these points.

Precipitation occurs with the first addition of $50 \mathrm{wt} \% \mathrm{NaOH}$. The first and second target $\mathrm{pH}$ points proved difficult to achieve since they both fell near the equivalence point of the reaction. The $\mathrm{pH}$ for each point was monitored with a broad range $\mathrm{pH}$ test strip. At $\mathrm{pH} 7$, it is notable that the material becomes a very thick slurry as shown in Figure 3-6C. This precipitate is thick enough that the beaker can be turned upside down without spilling any material. The major component of the precipitate at $\mathrm{pH} 7$ is $\mathrm{Al}(\mathrm{OH})_{3}$ solid. With the addition of additional sodium hydroxide, the reaction quickly moves past this point and the aluminum forms a more soluble species, presumed to be the anionic $\mathrm{Al}(\mathrm{OH})_{4}{ }^{-45}$

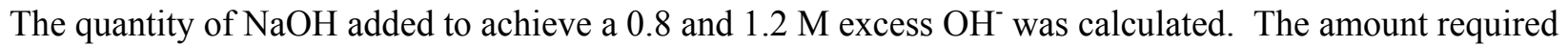
to complex all $\mathrm{Al}$ in the simulant was accounted for in the calculation. The slurry produced at the final neutralization point for both 0.8 and $1.2 \mathrm{M}$ excess $\mathrm{OH}^{-}$was visually similar. These points are represented in Figure 3-6D.

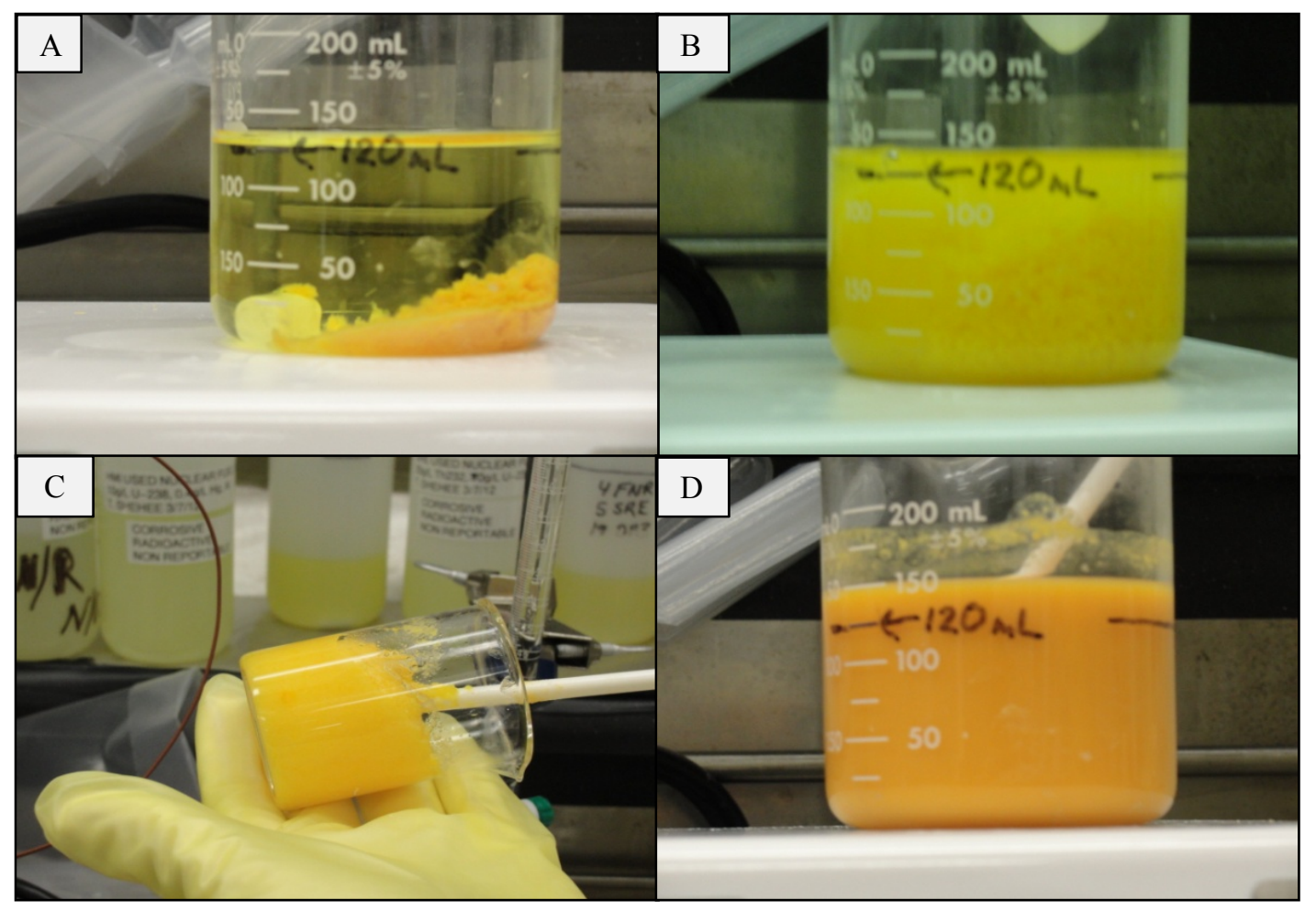

Figure 3-6. Neutralization of simulated dissolver batch. A) after addition of a few $\mathrm{mL} 50 \mathrm{wt} \%$ NaOH showing initial precipitation; B) neutralization at $\mathrm{pH} \mathrm{4.5;} \mathrm{C)} \mathrm{neutralization} \mathrm{at} \mathrm{pH} 7$; D) neutralization with excess 50 wt \% $\mathrm{NaOH}$. 


\subsection{Physical Properties}

The density and mass fraction of the supernate, solids in the slurry, and the undissolved solids in the 14,000 L dissolver fluids are provided in Table 3-19. Note that the sample names follow the format \#F\#S\#D-M where the \# indicates the number of L-Bundles containing the F (FNR or Ford Nuclear Reactor), S (SRE or Sodium Reactor Experiment), or D (DR-3 or Denmark Reactor) fuel, and M indicates the amount of free hydroxide molarity at the end of neutralization $(0.8 \mathrm{M}$ or $1.2 \mathrm{M})$. (Note: These solutions may also contain DU to lower the ${ }^{235} \mathrm{U}$ isotopic percentage.) Tables 3-13 through 3-14 provide Equations used for flow calculations. The rheological data for the $14,000 \mathrm{~L}$ dissolver fluids are provided in Table 3-20 with data fitted to the Newtonian and Bingham Plastic models as well as the apparent viscosity at $600 \mathrm{~s}^{-1}$. These tables provide the average physical property values and the $95 \%$ confidence level of the average physical property values.

For the 14,000 L dissolver fluids, subsamples were pulled, allowed to settle for 24 hours, decanted and the resulting slurries analyzed for density and total solids to see if there was an effect on slurry concentration in the determination of the undissolved solids properties. These results are provided in Table 3-11 and they clearly show that by concentrating the slurry via settling decanting, the UDS density deceases and the confidence level decreased, in all cases. A reason for the larger calculated densities and errors is the denominator in Equations 7 and 8, $\rho_{\text {sup }}+\rho_{s}\left(f_{u d s}-1\right)$. For a low UDS slurry, the density of the supernate and slurry have values that are close to each other and are made closer by further reducing the slurry density when considering the UDS fraction. This difference can be very small, thereby increasing the UDS density and error.

Table 3-11 Undissolved Solids Density Determination Using Concentrated 14,000 L Dissolver Fluids

\begin{tabular}{|c|c|c|c|c|c|c|c|c|}
\hline \multirow{3}{*}{ Identification } & \multicolumn{4}{|c|}{ As-Received Slurry } & \multicolumn{4}{|c|}{ Concentrated Slurry } \\
\hline & Slurry & & UDS & & Slurry & & UDS & \\
\hline & $\begin{array}{c}\text { Density } \\
(\mathrm{g} / \mathrm{ml})\end{array}$ & $\begin{array}{c}\text { TS } \\
\text { fraction }\end{array}$ & $\begin{array}{l}\text { Density } \\
\left(\mathrm{g} / \mathrm{cm}^{3}\right)\end{array}$ & STDEV & $\begin{array}{c}\text { Density } \\
(\mathrm{g} / \mathrm{ml})\end{array}$ & $\begin{array}{c}\text { TS } \\
\text { fraction }\end{array}$ & $\begin{array}{l}\text { Density } \\
\left(\mathrm{g} / \mathrm{cm}^{3}\right)\end{array}$ & STDEV \\
\hline $\begin{array}{c}\text { 4FS19D- } \\
0.8 \mathrm{M}\end{array}$ & 1.3057 & 0.3322 & 14.9481 & 4.7964 & 1.3336 & 0.3907 & 2.647 & 0.090 \\
\hline $\begin{array}{c}\text { 4FS19D- } \\
1.2 \mathrm{M}\end{array}$ & 1.3103 & 0.3527 & 3.5842 & 0.2617 & 1.3347 & 0.3750 & 3.301 & 0.147 \\
\hline 8S19D-0.8M & 1.3180 & 0.3422 & 8.8419 & 0.5403 & 1.3495 & 0.4033 & 2.763 & 0.041 \\
\hline 8S19D-1.2M & 1.3207 & 0.3418 & 10.4840 & 1.3970 & 1.3687 & 0.3993 & 3.429 & 0.074 \\
\hline 5SRE-0.8M & 1.1477 & 0.1751 & 7.2870 & 0.8528 & 1.1769 & 0.2031 & 5.686 & 0.512 \\
\hline 5SRE-1.2M & 1.1601 & 0.1840 & 7.0746 & 0.4654 & 1.2070 & 0.2285 & 5.029 & 0.390 \\
\hline 10SRE-0.8M & 1.2397 & 0.2686 & 5.2041 & 0.2903 & 1.2487 & 0.3030 & 2.691 & 0.147 \\
\hline 10SRE-1.2M & 1.2517 & 0.2741 & 5.7577 & 0.2181 & 1.2862 & 0.3188 & 3.352 & 0.199 \\
\hline
\end{tabular}

During the rheological measurements of 14,000 L dissolver fluids, all the SRE samples started to visually phase separate, where a clear liquid (supernate) was observed on the top surface of the rotating bob. This behavior was not the case for the other 14,000 L dissolver fluids or for the $12,000 \mathrm{~L}$ dissolver fluids.

All the flow curves were fitted to both Newtonian (N) and Bingham Plastic (BP) rheological models. A representative flow curve for the 14,000 L and 12,000 L dissolver fluids are shown in Figure 3-7, fitted to both the $\mathrm{N}$ and BP models. The 14,000 L flow curve shows that using a Newtonian fit the data is very 
similar to the BP fit; hence, the Newtonian results will be used to assess these fluids in the various calculations in this document. For the more viscous 12,000 L dissolver fluids, the Newtonian model can also be used, but with caution and depending on the condition of flow (e.g. laminar, turbulent). If the flow is turbulent, the Newtonian model is used for the pressure drop calculations, given that turbulent losses maybe greater for Newtonian fluids given that pipe roughness is not considered in the nonNewtonian case.

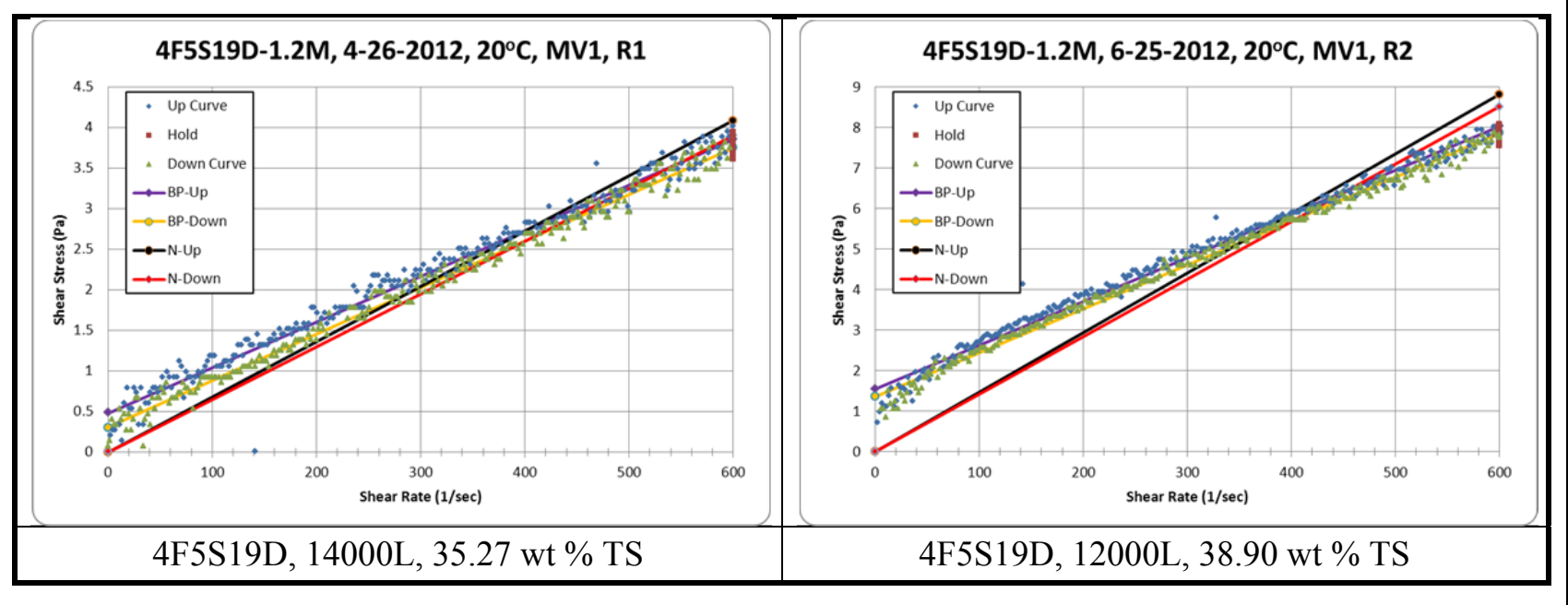

Figure 3-7 Flow Curves for 14,000 and 12,000 L 4F5S19D Slurries

Particle size distributions were obtained for the 12,000 L dissolver fluids and the distributions are shown in Figure 3-8 and have similar PSD profiles. The volume percentiles, mean values and the average and standard deviations are provided in Table 3-12. The deposition velocity correlation (Equation 32) uses the $85^{\text {th }}$ percentile data and since this data is not available, the $90^{\text {th }}$ percentile data will be used and is conservative. The mean volume of 19.3 microns is used to determine if the slurry can be treated as a noncohesive slurry and, if so, its mass flux is determined.

Table 3-12 Particle Size Distribution, Percentiles and Mean Values

\begin{tabular}{|c|c|c|c|c|c|c|c|}
\hline \multirow{2}{*}{\multicolumn{2}{|c|}{ Parameter }} & \multicolumn{6}{|c|}{ Microns } \\
\hline & & \multirow{2}{*}{$\begin{array}{c}\text { 4F5S19D } \\
0.8 \mathrm{M} \\
1.58\end{array}$} & \multirow{2}{*}{$\begin{array}{c}4 \mathrm{~F} 5 \mathrm{~S} 19 \mathrm{D} \\
1.2 \mathrm{M} \\
1.64\end{array}$} & \multirow{2}{*}{$\begin{array}{c}8 \mathrm{~S} 19 \mathrm{D} \\
0.8 \mathrm{M} \\
2.15\end{array}$} & \multirow{2}{*}{$\begin{array}{c}8 \mathrm{~S} 19 \mathrm{D} \\
1.2 \mathrm{M} \\
1.75\end{array}$} & \multirow{2}{*}{$\begin{array}{c}\text { Average } \\
\text { Value }\end{array}$} & \multirow{2}{*}{$\begin{array}{c}\text { STDEV } \\
0.26\end{array}$} \\
\hline \multirow{10}{*}{ 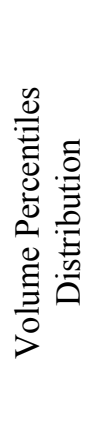 } & 10 & & & & & & \\
\hline & 20 & 3.75 & 3.77 & 4.91 & 4.10 & 4.13 & 0.55 \\
\hline & 25 & 5.08 & 4.83 & 6.16 & 5.21 & 5.32 & 0.58 \\
\hline & 40 & 9.16 & 7.99 & 10.43 & 8.85 & 9.11 & 1.01 \\
\hline & 50 & 12.82 & 10.79 & 14.57 & 12.49 & 12.67 & 1.55 \\
\hline & 60 & 16.77 & 14.57 & 19.60 & 16.91 & 16.96 & 2.06 \\
\hline & 70 & 21.00 & 19.12 & 25.94 & 22.28 & 22.09 & 2.88 \\
\hline & 75 & 23.56 & 21.89 & 30.00 & 25.72 & 25.29 & 3.51 \\
\hline & 90 & 39.23 & 37.46 & 50.34 & 43.57 & 42.65 & 5.73 \\
\hline & 95 & 56.58 & 52.11 & 67.83 & 56.92 & 58.36 & 6.68 \\
\hline \multirow{3}{*}{ 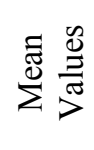 } & Volume & 19.33 & 16.74 & 22.02 & 18.88 & 19.24 & 2.17 \\
\hline & Number & 0.46 & 0.40 & 0.49 & 0.45 & 0.45 & 0.04 \\
\hline & Area & 4.21 & 3.92 & 4.93 & 4.40 & 4.36 & 0.43 \\
\hline
\end{tabular}




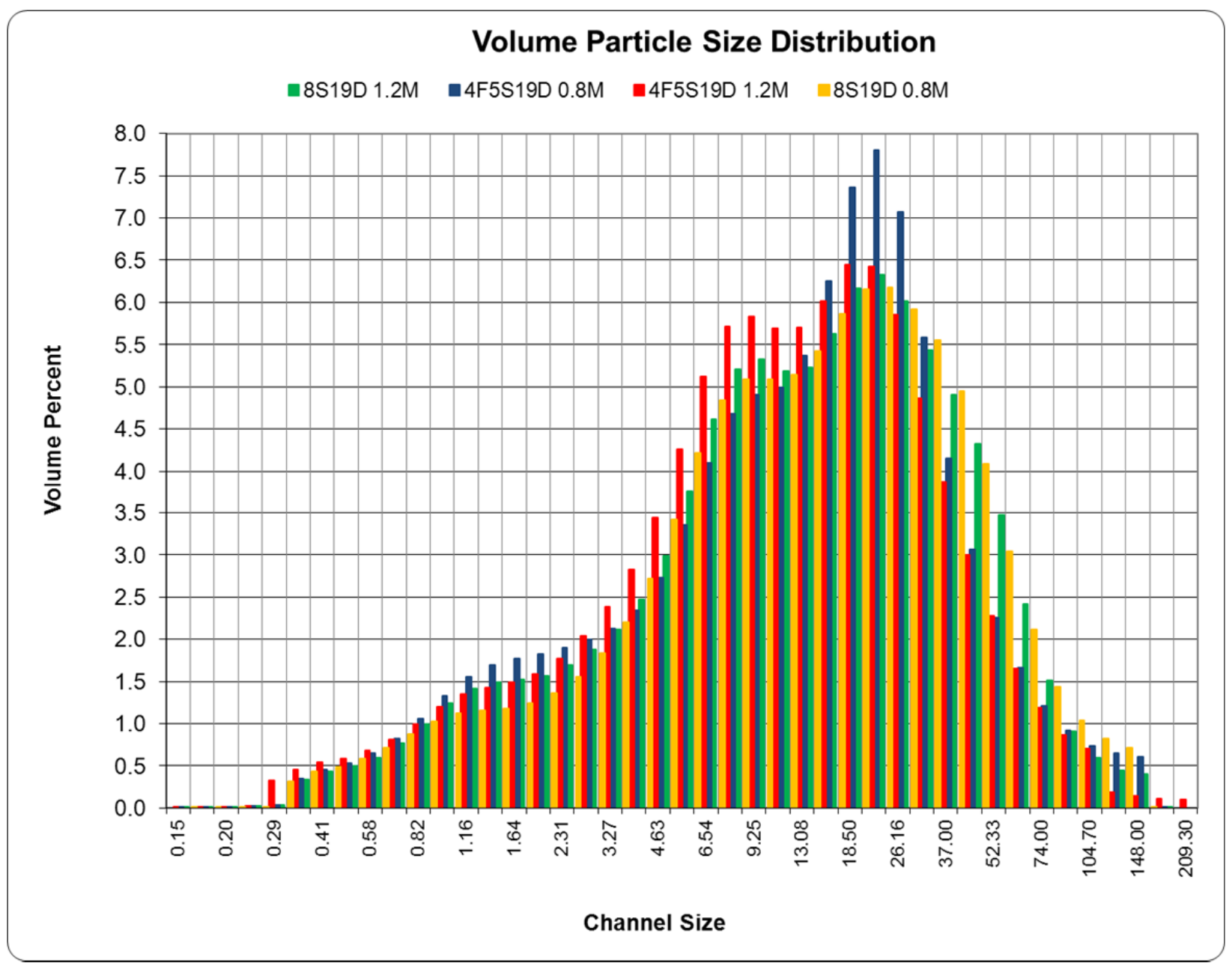

Figure 3-8 Particle Size Distribution of 12,000 L Dissolver Batch Fluids

\subsection{Flow Data}

The properties of water with a density of $62.4 \mathrm{lbm} / \mathrm{ft}^{3}$ (specific gravity $(\mathrm{SG})=1$ ) and viscosity of $1 \mathrm{cp}=$ $0.00674 \mathrm{lbm} / \mathrm{ft} \cdot \mathrm{sec}$ and a dissolver fluid (slurry) with a SG of 1.148 and viscosity of $14.5 \mathrm{cp}$ were used with the Equations shown in Table 3-13 represents the energy Equation for the complete waste transfer line associated with the 3-inch piping between Building 221-H and the HPP. Table 3-14 represents the energy Equation for the 3-inch header in Building 221-H that includes only the initial section of the waste transfer line having a slope of $0.005 \mathrm{ft}_{\text {vertical }} / \mathrm{ft}_{\text {horizontal }}$ leaving Building $221-\mathrm{H}$. 
Table 3-13. Energy Equations for Velocity Determination for Waste Transfer Lines Between Building 221-H and HPP

\begin{tabular}{|c|c|}
\hline $\begin{array}{c}\text { WH\#/Line Number } \\
(\text { HPP\#6) }\end{array}$ & $17.1=\frac{V^{2}}{2 g}\left(1.5+3216 f_{T}\right)$ \\
\hline $\begin{array}{c}\text { WH \#4/WF1101 } \\
(\mathrm{HPP} 45)\end{array}$ & $15.3=\frac{V^{2}}{2 g}\left(1.5+3340 f_{T}\right)$ \\
\hline $\begin{array}{c}\mathrm{WH} \# 3 / \mathrm{WF} 1102 \\
(\mathrm{HPP} 5)\end{array}$ & $13.9=\frac{V^{2}}{2 g}\left(1.5+3332 f_{T}\right)$ \\
\hline $\begin{array}{c}\mathrm{WH} \# 2 / \mathrm{WF} 1103 \\
(\mathrm{HPP} 6)\end{array}$ & $12.8=\frac{V^{2}}{2 g}\left(1.5+3159 f_{T}\right)$ \\
\hline
\end{tabular}

Table 3-14. Energy Equations for Velocity Determination for Initial Section of the Waste Transfer Lines Leaving Building 221-H with Slope of 0.005

\begin{tabular}{|c|c|}
\hline WH\#/Line Number & Energy Equation \\
\hline WH \#1/WF1100 & $4.91=\frac{V^{2}}{2 g}\left(1.5+588 f_{T}\right)$ \\
\hline WH \#4/WF1101 & $3.12=\frac{V^{2}}{2 g}\left(1.5+575 f_{T}\right)$ \\
\hline WH \#3/WF1102 & $1.71=\frac{V^{2}}{2 g}\left(1.5+575 f_{T}\right)$ \\
\hline WH \#2/WF1103 & $0.64=\frac{V^{2}}{2 g}\left(1.5+531 f_{T}\right)$ \\
\hline
\end{tabular}

Table 3-15. Average Velocity, Reynolds Number, and Flow rate for Waste Transfer Lines Between Building 221-H and HPP

\begin{tabular}{|c|c|c|c|c|c|c|c|c|c|c|c|c|}
\hline \multirow{4}{*}{ Line } & \multicolumn{6}{|c|}{$\begin{array}{c}\text { Dissolver Slurry } \\
14.5 \mathrm{cP}, 1.148 \mathrm{~g} / \mathrm{mL} \\
\end{array}$} & \multicolumn{6}{|c|}{$\begin{array}{c}\text { Water } \\
1 \mathrm{cP}, 1 \mathrm{~g} / \mathrm{mL}\end{array}$} \\
\hline & \multicolumn{6}{|c|}{ Pipe roughness } & \multicolumn{6}{|c|}{ Pipe roughness } \\
\hline & \multicolumn{3}{|c|}{$0.0015 \mathrm{ft}$} & \multicolumn{3}{|c|}{$0.006667 \mathrm{ft}$} & \multicolumn{3}{|c|}{$0.0015 \mathrm{ft}$} & \multicolumn{3}{|c|}{$0.006667 \mathrm{ft}$} \\
\hline & $\begin{array}{c}\mathrm{V} \\
(\mathrm{ft} / \mathrm{s})\end{array}$ & $\mathrm{N}_{\mathrm{RE}}$ & $\begin{array}{c}\mathrm{Q} \\
(\mathrm{gpm})\end{array}$ & $\begin{array}{c}\mathrm{V} \\
(\mathrm{ft} / \mathrm{s})\end{array}$ & $\mathrm{N}_{\mathrm{RE}}$ & $\begin{array}{c}\mathrm{Q} \\
(\mathrm{gpm})\end{array}$ & $\begin{array}{c}\mathrm{V} \\
(\mathrm{ft} / \mathrm{s})\end{array}$ & $\mathrm{N}_{\mathrm{RE}}$ & $\begin{array}{c}\mathrm{Q} \\
(\mathrm{gpm})\end{array}$ & $\begin{array}{c}\mathrm{V} \\
(\mathrm{ft} / \mathrm{s})\end{array}$ & $\mathrm{N}_{\mathrm{RE}}$ & $\begin{array}{c}\mathrm{Q} \\
(\mathrm{gpm})\end{array}$ \\
\hline WF1100 & 3.07 & 5848 & 70.7 & 2.78 & 5216 & 64.1 & 4.22 & 99903 & 97.3 & 3.15 & 74586 & 72.6 \\
\hline WF1101 & 2.81 & 5260 & 64.7 & 2.57 & 4807 & 59.1 & 3.89 & 92154 & 89.7 & 2.92 & 69198 & 67.4 \\
\hline WF1102 & 2.65 & 4975 & 61.2 & 2.43 & 4559 & 56.1 & 3.69 & 87435 & 85.1 & 2.78 & 65912 & 64.2 \\
\hline WF1103 & 2.61 & 4896 & 60.2 & 2.40 & 4490 & 55.2 & 3.64 & 86094 & 83.8 & 2.75 & 64994 & 63.3 \\
\hline
\end{tabular}


SRNL-STI-2012-00279

Revision 1

Table 3-16. Average Velocity, Reynolds Number, and Flow rate for Initial Section of the Waste Transfer Lines Leaving Building 221-H With Slope of 0.005

\begin{tabular}{|c|c|c|c|c|c|c|c|c|c|c|c|c|}
\hline \multirow{4}{*}{ Line } & \multirow{2}{*}{\multicolumn{6}{|c|}{$\begin{array}{c}\text { Dissolver Fluid } \\
14.5 \mathrm{cP}, 1.148 \mathrm{~g} / \mathrm{mL} \\
\text { Pipe roughness }\end{array}$}} & \multirow{2}{*}{\multicolumn{6}{|c|}{$\begin{array}{c}\text { Water } \\
1 \mathrm{cP}, 1 \mathrm{~g} / \mathrm{mL} \\
\text { Pipe roughness }\end{array}$}} \\
\hline & & & & & & & & & & & & \\
\hline & \multicolumn{3}{|c|}{$0.0015 \mathrm{ft}$} & \multicolumn{3}{|c|}{$0.006667 \mathrm{ft}$} & \multicolumn{3}{|c|}{$0.0015 \mathrm{ft}$} & \multicolumn{3}{|c|}{$0.006667 \mathrm{ft}$} \\
\hline & $\begin{array}{c}\mathrm{V} \\
(\mathrm{ft} / \mathrm{s})\end{array}$ & $\mathrm{N}_{\mathrm{RE}}$ & $\begin{array}{c}\mathrm{Q} \\
(\mathrm{gpm})\end{array}$ & $\begin{array}{c}\mathrm{V} \\
(\mathrm{ft} / \mathrm{s})\end{array}$ & $\mathrm{N}_{\mathrm{RE}}$ & $\begin{array}{c}\mathrm{Q} \\
(\mathrm{gpm})\end{array}$ & $\begin{array}{c}\mathrm{V} \\
(\mathrm{ft} / \mathrm{s})\end{array}$ & $\mathrm{N}_{\mathrm{RE}}$ & $\begin{array}{c}\mathrm{Q} \\
(\mathrm{gpm})\end{array}$ & $\begin{array}{c}\mathrm{V} \\
(\mathrm{ft} / \mathrm{s} \\
)\end{array}$ & $\mathrm{N}_{\mathrm{RE}}$ & Q (gpm) \\
\hline WF1100 & 3.84 & 7192 & 88.4 & 3.47 & 6507 & 80.0 & 5.10 & 120636 & 117.4 & 3.84 & 90990 & 88.6 \\
\hline WF1101 & 2.99 & 5612 & 69.0 & 2.74 & 5139 & 63.2 & 4.03 & 95380 & 92.8 & 3.09 & 73090 & 71.2 \\
\hline WF1102 & 2.12 & 3964 & 48.7 & 1.97 & 3685 & 45.3 & 2.90 & 68716 & 66.9 & 2.27 & 53831 & 52.4 \\
\hline WF1103 & 1.25 & 2334 & 28.7 & 1.18 & 2212 & 27.2 & 1.76 & 41646 & 40.5 & 1.43 & 33825 & 32.9 \\
\hline
\end{tabular}

The deposition velocity for the $90^{\text {th }}$ percentile particle, the dimensionless grain diameter, and the pipe fill ratio (based on inside diameter of pipe), velocity and Reynolds number for the minimum and maximum pipe slopes are provided in Table 3-17 for a nominal $25 \mathrm{gpm}$ flowrate for all the dissolver batches using the values inTable 3-19 and Table 3-20. For the 14,000 L dissolver batches all the fluids were treated as Newtonian and for the $12,000 \mathrm{~L}$ dissolver batches all the fluids were treated as Newtonian and nonNewtonian in determining the fill ratio, velocity and Reynolds number. For the non-Newtonian cases, if the slope was inadequate, a slope that can process $25 \mathrm{gpm}$ of fluid is provided. A calculation was performed using water to determine the transfer velocity required to exceed the deposition velocity when using water and this was $57.5 \mathrm{gpm}$. 
Table 3-17 Deposition, Dimensionless Grain Diameter, Fill Ratio, Velocity and Reynolds Number for the Minimum and Maximum Pipe Slopes at $25 \mathrm{gpm}$

\begin{tabular}{|c|c|c|c|c|c|c|c|c|c|c|}
\hline \multirow{4}{*}{ 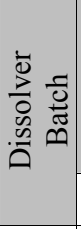 } & \multirow{3}{*}{ Sample } & \multirow{3}{*}{$\begin{array}{l}\text { Deposition } \\
\text { Velocity } \\
(\mathrm{ft} / \mathrm{s})\end{array}$} & \multirow{3}{*}{$\mathrm{d}_{\mathrm{gr}} *$} & \multicolumn{7}{|c|}{25 GPM Sloped Data } \\
\hline & & & & & \multicolumn{3}{|c|}{ Slope $=0.005 \mathrm{ft} / \mathrm{ft}$} & \multicolumn{3}{|c|}{ Slope $=0.0268 \mathrm{ft} / \mathrm{ft}$} \\
\hline & & & & Model** & $\begin{array}{c}\text { Fill } \\
\text { Ratio }\end{array}$ & $\begin{array}{c}\text { Vel. } \\
(\mathrm{ft} / \mathrm{sec})\end{array}$ & $\mathrm{Re}$ & $\begin{array}{c}\text { Fill } \\
\text { Ratio }\end{array}$ & $\begin{array}{c}\text { Vel. } \\
(\mathrm{ft} / \mathrm{sec})\end{array}$ & $\operatorname{Re}$ \\
\hline & Water & 4.44 & 0.56 & $\mathrm{~N}$ & 0.548 & 1.93 & 48406 & 0.331 & 3.76 & 65827 \\
\hline \multirow{8}{*}{$\begin{array}{l}1 \\
8 \\
8 \\
\pm \\
\pm\end{array}$} & 4FS19D-0.8M & 3.71 & 0.19 & $\mathrm{~N}$ & 0.606 & 1.71 & 10738 & 0.354 & 3.42 & 15021 \\
\hline & 4FS19D-1.2M & 3.71 & 0.16 & $\mathrm{~N}$ & 0.617 & 1.67 & 8597 & 0.359 & 3.36 & 12092 \\
\hline & 8S19D-0.8M & 3.67 & 0.20 & $\mathrm{~N}$ & 0.599 & 1.73 & 12254 & 0.352 & 3.45 & 17091 \\
\hline & 8S19D-1.2M & 3.68 & 0.18 & $\mathrm{~N}$ & 0.611 & 1.70 & 9737 & 0.356 & 3.39 & 13653 \\
\hline & 5SRE-0.8M & 4.08 & 0.24 & $\mathrm{~N}$ & 0.591 & 1.76 & 14669 & 0.348 & 3.50 & 20378 \\
\hline & 5SRE-1.2M & 4.04 & 0.25 & $\mathrm{~N}$ & 0.589 & 1.77 & 15278 & 0.348 & 3.51 & 21206 \\
\hline & 10SRE-0.8M & 3.87 & 0.17 & $\mathrm{~N}$ & 0.616 & 1.68 & 8796 & 0.359 & 3.36 & 12365 \\
\hline & 10SRE-1.2M & 3.83 & 0.11 & $\mathrm{~N}$ & 0.606 & 1.71 & 10679 & 0.355 & 3.42 & 14941 \\
\hline \multirow{8}{*}{$\begin{array}{l}1 \\
8 \\
8 \\
1 \\
1\end{array}$} & \multirow{2}{*}{ 4FS19D-0.8M } & \multirow{2}{*}{3.69} & \multirow{2}{*}{0.11} & $\mathrm{~N}$ & 0.658 & 1.56 & 4449 & 0.375 & 3.16 & 6380 \\
\hline & & & & $\mathrm{NN}$ & \multicolumn{3}{|c|}{0.0098 (slope required) } & 0.330 & 3.78 & 10193 \\
\hline & \multirow{2}{*}{ 4FS19D-1.2M } & \multirow{2}{*}{3.66} & \multirow{2}{*}{0.10} & $\mathrm{~N}$ & 0.666 & 1.53 & 3937 & 0.379 & 3.13 & 5670 \\
\hline & & & & $\mathrm{NN}$ & \multicolumn{3}{|c|}{0.0092 (slope required) } & 0.327 & 3.82 & 8347 \\
\hline & \multirow{2}{*}{ 8S19D-0.8M } & \multirow{2}{*}{3.77} & \multirow{2}{*}{0.12} & $\mathrm{~N}$ & 0.645 & 1.59 & 5395 & 0.370 & 3.22 & 7688 \\
\hline & & & & $\mathrm{NN}$ & \multicolumn{3}{|c|}{0.0061 (slope required) } & 0.321 & 3.92 & 10711 \\
\hline & \multirow{2}{*}{ 8S19D-1.2M } & \multirow{2}{*}{3.69} & \multirow{2}{*}{0.16} & $\mathrm{~N}$ & 0.621 & 1.66 & 8040 & 0.361 & 3.34 & 11329 \\
\hline & & & & $\mathrm{NN}$ & 0.549 & 1.93 & 11047 & 0.315 & 4.02 & 15466 \\
\hline
\end{tabular}

$* \mathrm{~d}_{\mathrm{gr}}=$ dimensionless grain diameter

** Model can be either Newtonian $(\mathrm{N})$ or Non-Newtonian $(\mathrm{NN})$ to determine the fill ratio, velocity or Reynolds number.

Additional calculations were performed on the $1^{\text {st }}$ section of piping to determine if there is a potential for the fluids to start accumulating into their respective header in Building 221-H. The energy equations in Table 3-14 and the physical properties of the 12,000 L 4FS19D-0.8M fluid are used and the results are provided in Table 3-18.

Table 3-18 Maximum Flow using the Most non-Newtonian Solution, 12000 L 4FS19D-0.8M

\begin{tabular}{|c|c|}
\hline Line & Flow (gpm) \\
\hline $\begin{array}{c}\text { WF1100 } \\
\text { (WH 1) }\end{array}$ & 109.8 \\
\hline $\begin{array}{c}\text { WF1101 } \\
\text { (WH 4) }\end{array}$ & 86.0 \\
\hline $\begin{array}{c}\text { WF1102 } \\
\text { (WH 3) }\end{array}$ & 52.4 \\
\hline $\begin{array}{c}\text { WF1103 } \\
\text { (WH 2) }\end{array}$ & 1.7 \\
\hline
\end{tabular}


Table 3-19. Supernate, Slurry, and Undissolved Density and Solids Fraction Data, 14,000 L and 12,000 L Dissolver Batch Fluids

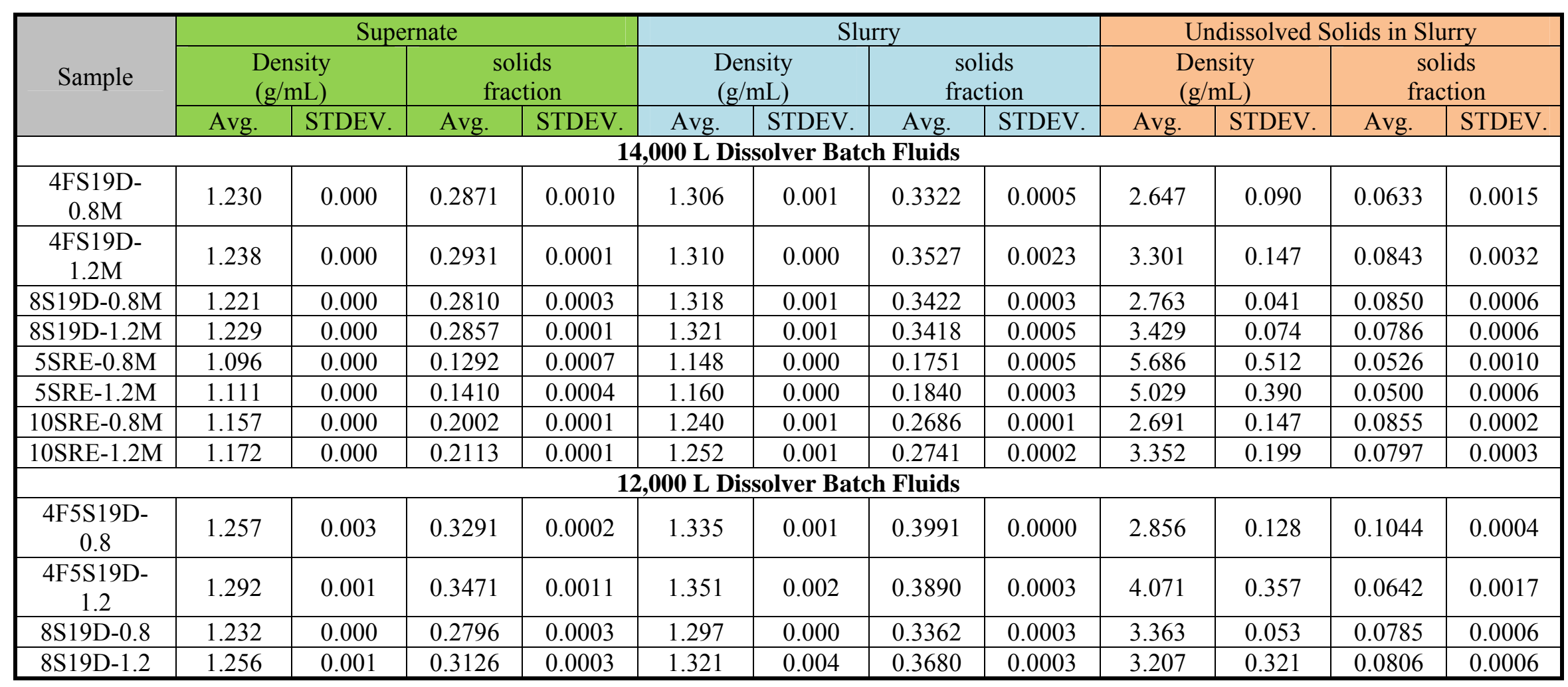


Table 3-20. Rheological Data, 14,000 L and 12,000 L Dissolver Batch Fluids

\begin{tabular}{|c|c|c|c|c|c|c|c|c|c|c|c|c|c|c|}
\hline \multirow{4}{*}{ Sample } & \multirow{2}{*}{\multicolumn{4}{|c|}{$\begin{array}{c}\text { Newtonian Fluid } \\
\text { Viscosity (cP) }\end{array}$}} & \multirow{3}{*}{\multicolumn{2}{|c|}{$\begin{array}{c}\text { Apparent } \\
\text { Viscosity } \\
\text { At Maximum } \\
\text { Shear rate cP }\end{array}$}} & \multicolumn{8}{|c|}{ Bingham Plastic } \\
\hline & & & & & & & \multicolumn{4}{|c|}{ Up Curve } & \multicolumn{4}{|c|}{ Down Curve } \\
\hline & \multicolumn{2}{|c|}{ Up Curve } & \multicolumn{2}{|c|}{ Down Curve } & & & \multicolumn{2}{|c|}{ Yield Stress $(\mathrm{Pa})$} & \multicolumn{2}{|c|}{$\begin{array}{c}\text { Plastic Viscosity } \\
(\mathrm{cP})\end{array}$} & \multicolumn{2}{|c|}{$\begin{array}{l}\text { Yield Stress } \\
(\mathrm{Pa})\end{array}$} & \multicolumn{2}{|c|}{$\begin{array}{c}\text { Plastic Viscosity } \\
(\mathrm{cP})\end{array}$} \\
\hline & Avg. & STDEV & Avg. & STDEV & Avg. & STDEV. & Avg. & STDEV & Avg. & STDEV & Avg. & STDEV & Avg. & STDEV \\
\hline \multicolumn{15}{|c|}{ 14,000 L Dissolver Batch Fluids } \\
\hline $\begin{array}{c}\text { 4F5S19D- } \\
0.8 \\
\end{array}$ & 5.5 & 0.2 & 5.3 & 0.3 & 5.3 & 0.2 & 0.12 & 0.16 & 4.80 & 0.02 & -0.01 & 0.02 & 5.11 & 0.06 \\
\hline $\begin{array}{c}\text { 4F5S19D- } \\
1.2\end{array}$ & 6.8 & 0.0 & 6.5 & 0.1 & 6.4 & 0.0 & 0.45 & 0.05 & 5.67 & 0.07 & 0.28 & 0.04 & 5.77 & 0.03 \\
\hline 8S19D-0.8 & 4.9 & 0.2 & 4.6 & 0.3 & 4.7 & 0.1 & 0.24 & 0.08 & 4.33 & 0.04 & -0.01 & 0.11 & 4.59 & 0.01 \\
\hline 8S19D-1.2 & 6.1 & 0.1 & 5.5 & 0.3 & 5.6 & 0.3 & 0.17 & 0.20 & 5.17 & 0.26 & 0.05 & 0.02 & 5.39 & 0.35 \\
\hline 5SRE-0.8 & 3.6 & 0.1 & 3.1 & 0.2 & 3.3 & 0.2 & 0.35 & 0.07 & 2.68 & 0.30 & 0.05 & 0.03 & 3.01 & 0.16 \\
\hline 5SRE-1.2 & 3.5 & 0.8 & 3.1 & 0.7 & 3.4 & 0.4 & 0.40 & 0.26 & 2.56 & 0.15 & 0.10 & 0.27 & 2.82 & 0.06 \\
\hline 10SRE-0.8 & 6.3 & 0.2 & 5.8 & 0.2 & 5.8 & 0.2 & 0.63 & 0.04 & 4.77 & 0.31 & 0.20 & 0.02 & 5.29 & 0.29 \\
\hline 10SRE-1.2 & 5.3 & 0.4 & 4.9 & 0.5 & 4.9 & 0.4 & 0.54 & 0.11 & 3.96 & 0.10 & 0.14 & 0.14 & 4.52 & 0.12 \\
\hline \multicolumn{15}{|c|}{ 12,000 L Dissolver Batch Fluids } \\
\hline $\begin{array}{c}\text { 4F5S19D- } \\
0.8\end{array}$ & 12.8 & 0.2 & 12.2 & 0.0 & 11.2 & 0.1 & 1.66 & 0.00 & 8.67 & 0.19 & 1.11 & 0.06 & 9.41 & 0.11 \\
\hline $\begin{array}{c}\text { 4F5S19D- } \\
1.2 \\
\end{array}$ & 14.6 & 0.2 & 14.2 & 0.0 & 13.0 & 0.1 & 1.49 & 0.08 & 10.81 & 0.00 & 1.37 & 0.01 & 10.80 & 0.06 \\
\hline 8S19D-0.8 & 10.4 & 0.2 & 10.0 & 0.2 & 9.5 & 0.2 & 0.92 & 0.09 & 8.14 & 0.01 & 0.61 & 0.03 & 8.48 & 0.16 \\
\hline 8S19D-1.2 & 7.3 & 0.1 & 7.1 & 0.0 & 6.8 & 0.2 & 0.51 & 0.22 & 5.80 & 0.17 & 0.43 & 0.25 & 6.00 & 0.00 \\
\hline
\end{tabular}




\subsection{Conclusions}

Dissolution time for Th was fast ( $<5 \mathrm{~min}$ ) in 7 and $10 \mathrm{M} \mathrm{HNO}_{3}$ when $0.05 \mathrm{M} \mathrm{F}^{-}$was present. At $10 \mathrm{M}$ $\mathrm{HNO}_{3}$, dissolution of $\mathrm{Al}$ proved to be extremely slow when $0.002 \mathrm{M} \mathrm{Hg}$ was used as is expected to be used in the dissolver. Dissolution was longer than 2 hours without reaching a complete dissolution. Conversely, when dissolved in one of the dissolver solutions from the earlier Th dissolution which started at $7 \mathrm{M} \mathrm{HNO}_{3}$, the aluminum dissolved in 4.5 to 7 minutes.

Hydrogen gas generation was generally low for thorium dissolutions. The highest raw volume percent of $1.10 \%$ in a Th dissolution came when the initial nitric acid concentration was low $(4 \mathrm{M})$ and there was no initial aluminum present in the solution. With aluminum pre-dissolved at about $0.3 \mathrm{M}$, an initial nitric acid solution of $7 \mathrm{M}$ produced about $1.70 \% \mathrm{H}_{2}$ raw measured volume percent. These values are lower than those observed in literature and are less than those shown in several of the Al dissolutions.

Neutralization of the simulants showed considerable formation of solid material. This material was still fluid at both neutralization points for the idealized dissolver batch composed of 4 FNR, 5 SRE, and 19 DR-3 L-Bundles or a batch made of 8 SRE and 19 DR-3 L-Bundles. The density of the material only increased upon further $\mathrm{NaOH}$ addition to achieve 1.2 M excess.

The surface area calculations for the L-Bundles along with the SRE, HMI, and DR-3 assemblies were completed. When comparing the total surface areas of these UNF assemblies with that of the prior processed MURR assemblies as shown in Figure 3-1, the outer surface areas of the SRE, HMI, and DR-3 assemblies are less. Since the off-gas generation rate from dissolving the L-Bundle with the assemblies inside is surface area dependent, dissolving the SRE, HMI, and DR-3 UNF in the H-Canyon is feasible and is bounded by the analyses provided in the prior off-gas calculations for MURR Assemblies. ${ }^{34}$

The literature revealed that about 5 moles of $\mathrm{HNO}_{3}$ are needed to dissolve 1 mole of thorium versus 3.75 mole of $\mathrm{HNO}_{3}$ to dissolve 1 mole of aluminum. The literature and experiments showed that the highest vol $\% \mathrm{H}_{2}$ in the off-gas from the dissolution of thorium metal was $3.6 \mathrm{wt} \%$ on a water free basis whereas the nominal vol $\% \mathrm{H}_{2}$ in the off-gas from the dissolution of aluminum metal was $7 \mathrm{wt} \%$ on a water free basis. The thorium dissolution experiments and literature reviewed showed that at lower $\mathrm{HNO}_{3}$ concentrations $(<7 \mathrm{M})$ the dissolved $\mathrm{Al}$ has a negligible impact on the dissolution of thorium but this is not true at high $\mathrm{HNO}_{3}$ concentrations (around $10 \mathrm{M}$ ). The amount of $\mathrm{HNO}_{3}$ and dissolved thorium in solution has a significant impact on the thorium dissolution rate. The thorium dissolution rate is approximately second order with respect to the $\mathrm{HNO}_{3}$ concentration as shown in Figure 3-3. The $\mathrm{HNO}_{3}$ concentration also has a big impact on the aluminum dissolution as seen for the MURR and other High Aluminum-Low Uranium assemblies. The thorium dissolution rate, at a constant $\mathrm{HNO}_{3}$ concentration, drops as the amount of dissolved thorium increases as shown in Figure 3-5. The Al dissolution rate follows a similar behavior as the amount of dissolved aluminum increases as seen in the prior calculations for the dissolution of the MURR assemblies. ${ }^{34}$

There was concern about the off-gas generation from the SRE assemblies which have thorium fuel sources unlike the HMI and DR-3 which have High Aluminum-Low Uranium fuel sources. However, since the SRE assemblies are encased in an external aluminum bundling tube which is then encased in the aluminum L-Bundle, the peak off-gas rates are based on the same Al dissolution chemistry used for the MURR, HMI, and DR-3 assemblies. Should the inner thorium fuel tubes of the SRE assemblies become exposed, the experimental off-gas generation rates per unit surface area of thorium is 0.03 to $0.05 \mathrm{scfm} / \mathrm{ft}^{2}$ which is low compared to the off-gas generation rates per unit surface area of aluminum of $0.676 \mathrm{scfm} / \mathrm{ft}^{2}$. As a conservative measure, when the off-gas generation rates were calculated for the inner thorium fuel sources for the SRE assemblies, the Al dissolution off-gas rate was used and the off-gas generation was 
still small compared to the off-gas generation from dissolving the exterior L-Bundle and the exterior bundling tube of the SRE assemblies.

The peak off-gas rates for the FNR, SRE, HMI and DR-3 were determined to be bounded by the controls listed in prior calculations for the dissolution of the MURR assemblies. ${ }^{34}$ Initially, up to 4 bundles of FNR, SRE, HMI, and/or DR-3 can be charged to a solution of fresh nitric acid. After the first dissolution, the number of bundles that can be charged is dependent on the concentration of aluminum in the dissolver solution as shown in Figure 8 and Table 8 of prior calculations for the dissolution of the MURR assemblies $^{34}$. Since there is conservatism built into these calculations, the proposed dissolver scenarios discussed above should be safe with respect to the $\mathrm{H}_{2}$ LFL.

The simulated 14,000 L dissolver batch fluids were visually very fluid in nature. The simulated 12,000 L dissolver batch fluid were also very fluid but could be modeled as a Bingham Plastic fluid. The density of the slurries ranged from 1.148 to $1.335 \mathrm{~g} / \mathrm{mL}$. The undissolved solids fraction concentrations in the slurries were between 0.05 to 0.1044 and the average densities of these solids ranged between 2.65 to $5.7 \mathrm{~g} / \mathrm{cm}^{3}$, though it is expected that the average density is around $3.5 \mathrm{~g} / \mathrm{cm}^{3}$. The rheological data indicated that the 12,000 L dissolver fluids have non-Newtonian properties and were analyzed using both Newtonian and Bingham Plastic rheological models. There are slight differences in the viscosities as determined using the Bingham Plastic model, Newtonian model, or the apparent viscosity at the maximum shear rate for the 14,000 L batches, but these differences start to become slightly larger for the $12,000 \mathrm{~L}$ batches. A viscosity of $14.5 \mathrm{cP}$ and density of $1.148 \mathrm{~g} / \mathrm{mL}$ were used to assess the hydraulics of the various waster lines. The analysis assumed that the lines were full of fluid with no air entrainment. The flow calculations showed that the initial section of the waste lines leaving Building 221- $\mathrm{H}$ had a minimum flow rate of $27.2 \mathrm{gpm}$ associated with WF1103 (WH\#2) waste line and is due to this line having essentially no vertical drop as compared to the other waste lines. The flow calculations showed that the other waste lines provide a flow of at least $45.3 \mathrm{gpm}$. These calculated flow rates were for dirty piping. For clean piping, the calculated flow rates are slightly larger. The calculations showed that the waste lines leaving Building 221-H could handle the 25 to 30 gpm flow that the jet pumps provide, other than waste line WF1103 (WH\#2) for the condition where the waste header would not backup into Building 221-H. A calculation was performed using the most non-Newtonian fluid (12,000 L 4F5S19D$0.8 \mathrm{M}$ ) for all the headers and only WF1103 (WH\#2) was determined to backup. The effect of pipe roughness impacts the flow rates and in this example the flow rates could be reduced by over $20 \%$ based on clean pipe conditions.

Critical velocities and dimensionless grain diameters were calculated. The dimensionless grain diameters were all less than 1, indicating that all these fluids should be treated as cohesive slurries. The critical velocity calculation ranged between 3.5 to $4.1 \mathrm{ft} / \mathrm{s}$, indicating that some of the particles will settle to the bottom of the pipe. This critical velocity range is consistent with previous calculations performed for FCanyon transfers. ${ }^{7}$ There is not sufficient data or literature to make a determination if and how much material can potentially settle. Issues such as the actual floc size, shape and composition are not known and the effect of the fine particles on hindering the settling of the larger particles would impact such calculations. Given a range of pipe slopes in the transfer lines, the steepest slopes have transfer velocities of the same values of the critical velocities, given the $25 \mathrm{gpm}$. Shallower slopes have lower transfer velocities. 


\subsection{Recommendations}

The processing of fuels described in this document will occur in 3 phases. Due to a desire to begin processing to deploy a SRE flowsheet as quickly as possible, SRNL has provided a flowsheet for HCanyon to charge HA/LU bundles (SRNL-STI-2012-00279 Rev. 0) as part of Phase 1. In June 2010, SRNL issued a flowsheet report (SRNL-STI-2010-00005) for dissolution of MURR UNF. This flowsheet can also be used for the dissolution of HA/LU plate fuels such as FNR fuel. No issues have been identified by SRNL which would preclude H-Canyon from charging the FNR fuel and subsequent charging and blending with SRE and DR-3 UNF (Phase 2). Phase 3 will begin with charging SRE fuel bundles to a fresh dissolver solution followed by a number of DR-3 bundles. Charging recommendations per phase are shown below.

\subsection{Phase 1-2}

In Phase 1, SRNL proposes the following flowsheet for charging of HA/LU fuel to support the initial SRE flowsheet.

- $\quad 6-7 \mathrm{M} \mathrm{HNO}_{3}$

- $\quad 0.5 \mathrm{~g} / \mathrm{L} \mathrm{Gd}$

- $0.002 \mathrm{M} \mathrm{Hg}$

- 48 to 72 hour dissolution

The dissolver would be prepared with the nitric acid and gadolinium solution. Mercury catalysts will be added to the dissolver to a concentration of $0.002 \mathrm{M}$. A total of 4 bundles of Ford Nuclear Reactor (FNR) each with 5 assemblies could be charged to the dissolver at an immersion depth of 54 inches as described in the MURR dissolution flowsheet. ${ }^{34}$ The mercuric nitrate catalyst could be added at the rate specified in the report for the first charge. Subsequent charges would not require additional mercury catalyst. The generation of hydrogen during dissolution would be bounded by the MURR analysis. Dissolution time should be 48 - 72 hours.

For Phase 2, the number of bundles of SRE fuel to be charged to the dissolver solution from Phase 1 will be based on the methodology explained in the dissolution of the MURR assemblies ${ }^{34}$ which depends on several factors including the immersion depth, the amount of dissolved aluminum, the amount of irradiation of the assemblies, and the amount of catalyst present. Prior to addition of the SRE UNF bundles to the dissolver, fluoride should be added to achieve a concentration of $0.05 \mathrm{M}$. The fluoride acts as a catalyst and is required in the dissolution of Th metal. Following the dissolution of the SRE bundles, the number of DR-3 fuel bundles charged to the dissolver will be based on the methodology explained for the dissolution of the MURR assemblies just like the SRE assemblies.

\subsection{Phase 3}

In Phase 3, SRNL proposes the following flowsheet for charging of SRE fuel to support the final SRE flowsheet.

- $6-7 \mathrm{M} \mathrm{HNO}_{3}$

- $0.05 \mathrm{M} \mathrm{F}^{-}$

- $\quad 0.5 \mathrm{~g} / \mathrm{L} \mathrm{Gd}$

- $0.002 \mathrm{M} \mathrm{Hg}$

- 48 to 72 hour dissolution

The dissolver would be prepared with the nitric acid, fluoride, and gadolinium solution. Fluoride acts as a catalyst and is required in the dissolution of Th metal in the SRE fuel. The mercuric nitrate catalyst 
should be added at the rate specified in the MURR dissolution flowsheet ${ }^{34}$ for the first charge. Subsequent charges will not require additional mercury or fluoride catalyst. The number of bundles of SRE fuel that can be charged to the dissolver will be determined using the methodology explained in the dissolution of the MURR assemblies ${ }^{34}$ which depends on several factors including the immersion depth, the amount of dissolved aluminum, the amount of irradiation of the assemblies, and the amount of catalyst present. The generation of hydrogen during dissolution will be bounded by the MURR analysis. Dissolution time should be 48-72 hours. Following dissolution of the SRE bundles, the number of DR-3 fuel bundles charged to the dissolver will be based on the methodology explained for the dissolution of the MURR assemblies just like the SRE assemblies.

\subsection{Additional DR-3 Fuel}

L-Area has identified several bundles of HEU DR-3 which are pitted. These bundles are also high aluminum fuels; however, the fuel core is composed of either $\mathrm{U}-\mathrm{Al}_{\mathrm{x}}$ or $\mathrm{U}_{3} \mathrm{O}_{8}-\mathrm{Al}$, (cermet fuel) not the $\mathrm{U}_{3} \mathrm{Si}_{2}-\mathrm{Al}$ alloy of the LEU DR-3 material. U-Al $\mathrm{X}_{\mathrm{x}}$ or $\mathrm{U}_{3} \mathrm{O}_{8}-\mathrm{Al}$ fuels have been evaluated at the Savannah River Laboratory for processing by $\mathrm{H}$-canyon. ${ }^{34,43,46}$ The Perkins report determined that the dissolving behavior of the $\mathrm{U}_{3} \mathrm{O}_{8}^{-} \mathrm{Al}$ fuel was similar to that of a $\mathrm{U}-\mathrm{Al}_{\mathrm{x}}$ alloy fuel with fast dissolution rates in $\mathrm{HNO}_{3}{ }^{-}$ $\mathrm{Hg}\left(\mathrm{NO}_{3}\right)_{2}$ solutions. $^{46}$

The U-Al ${ }_{x}$ DR-3 fuel elements basically have the same dimensions as the LEU DR-3 evaluated in the report; therefore, surface area per unit height will not change. As an example, one $\mathrm{U}-\mathrm{Al}_{\mathrm{x}} \mathrm{DR}-3$ assembly with 4 fuel tubes has dimensions for Tube 1: $62.5 \times 6.395 \times 0.146[\mathrm{~cm}]$, Tube 2: $62.5 \times 7.375 \times$ 0.146 [cm], Tube 3: $62.5 \times 8.355 \times 0.146$ [cm], and Tube 4: $62.5 \times 9.335 \times 0.146$ [cm]. Of note, several assemblies such as 12/049, 12/050, 12/051 do not have a Fuel Tube 1. This configuration will give a slightly higher surface area per unit height but this variance should not be a significant effect on the amount of off-gas since the L-area Bundle is the primary surface source.

The U-Al $\mathrm{x}_{\mathrm{x}}$ alloy of the specific HEU DR-3 fuel elements will not affect the off-gas calculations since the off-gas generation source term for the MURR, FNR, SRE, and DR-3 assemblies comes from the work of Caracciolo. $^{43}$ The Caracciolo off-gas rates were based on $16 \mathrm{wt} \% \mathrm{U}-84 \mathrm{wt} \% \mathrm{Al}$ fuel cores with an average $8.4 \mathrm{wt} \% \mathrm{U}$ when taking into account the complete fuel element including the Al cladding. The specific $\mathrm{U}-\mathrm{Al}_{\mathrm{x}}$ cited in the Appendix A for this fuel has about $6 \mathrm{wt} \% \mathrm{U}$ in the complete fuel element including $\mathrm{Al}$ cladding which is not considerably different from the Caracciolo fuels.

Another difference of this HEU U-Al ${ }_{x}$ DR-3 fuel is that 5 DR-3 assemblies are stacked one on top of another versus having 4 DR-3 assemblies stacked one on top of another inside a L-Area Bundle tube. This will not affect the surface area per unit height other than extending the surface area one more assembly in terms of height and thus will not affect the peak off-gas rate other than taking longer to dissolve.

Several of the pitted fuel assemblies are composed of a $\mathrm{U}_{3} \mathrm{O}_{8}-\mathrm{Al}$ fuel core. These fuel elements basically have the same dimensions as the LEU DR-3 evaluated in this report so surface area per unit height should not change significantly. Example dimensions of these assemblies are $62.5 \times 6.38 \times 0.151 \mathrm{~cm}, 62.5 \mathrm{x}$ $7.36 \times 0.151 \mathrm{~cm}, 62.5 \times 8.34 \times 0.151 \mathrm{~cm}$, and $62.5 \times 9.32 \times 0.151 \mathrm{~cm}$ for fuel tubes 1, 2, 3, and 4, respectively.

Similar to the $\mathrm{UAl}_{\mathrm{x}}$ fuels, the $\mathrm{U}_{3} \mathrm{O}_{8}$-Al version of the HEU DR-3 fuel elements will not affect the off-gas calculations since the off-gas generation source term for the MURR, FNR, SRE, and DR-3 assemblies 
comes from the work of Caracciolo. ${ }^{43}$ The Caracciolo off-gas rates were based on 16 wt \% U-84 wt \% Al fuel cores with average $8.4 \% \mathrm{U}$ in the complete fuel element including $\mathrm{Al}$ cladding.

The specific $\mathrm{U}_{3} \mathrm{O}_{8}$-Al fuel cited in the Appendix A for this HEU DR-3 fuel element has about 29 wt \% U in the complete fuel element including $\mathrm{Al}$ cladding which is significantly different than the $8.4 \mathrm{wt} \% \mathrm{U}$ Caracciolo value. ${ }^{43}$ However, the Caracciolo report states that for the average $14.6 \mathrm{wt} \%$ U Type $1 \mathrm{~B}$ fuel elements the dissolution time was $16 \mathrm{~h}$ versus $\sim 32 \mathrm{~h}$ for the average $8.4 \mathrm{wt} \% \mathrm{U}$ Type $1 \mathrm{~A}$ fuel elements, but the $8.4 \mathrm{wt} \% \mathrm{U}$ Type $1 \mathrm{~A}$ fuel elements gave a peak off-gas rate of $5 \mathrm{scfm}$ while the $14.6 \mathrm{wt} \% \mathrm{U}$ Type $1 \mathrm{~B}$ fuel elements gave a peak off-gas rate of $3.2 \mathrm{scfm}$. In other words, a higher wt \% U fuel element may dissolve faster but the peak off-gas rate is lower, probably due to the increased foaming at the metal-nitric acid surfaces. The work of W. C. Perkins discusses the dissolving of $\mathrm{U}_{3} \mathrm{O}_{8}$-Al fuel elements with about $60 \mathrm{wt} \% \mathrm{U}_{3} \mathrm{O}_{8}$ and $40 \mathrm{wt} \% \mathrm{Al}$ in the fuel core and about $40 \mathrm{wt} \% \mathrm{Al}$ cladding in the entire fuel element which is equivalent to $31 \mathrm{wt} \% \mathrm{U}$ in the complete fuel element including Al cladding. ${ }^{46}$ For the Perkins $\mathrm{U}_{3} \mathrm{O}_{8}-\mathrm{Al}$ fuel elements the dissolution rate is about $0.7 \mathrm{lb}(\mathrm{h} * \mathrm{ft})$ for a $0.001 \mathrm{M} \mathrm{Hg}$ and a $0.7 \mathrm{M} \mathrm{Al}$ dissolver solution which matches rates shown in Caracciolo for $16 \mathrm{wt} \% \mathrm{U}-84 \mathrm{wt} \%$ Al fuel cores. ${ }^{43,46}$

Based on the Caracciolo and Perkins references, the $\mathrm{U}_{3} \mathrm{O}_{8}$-Al fuel cores should not behave differently in terms of the dissolution and amount of off-gas produced than the $\mathrm{U}_{3} \mathrm{Si}_{2}$-Al DR-3 already discussed in the report.

\subsection{Flowrate recommendations}

It is recommended that Waste Header \#1, 4, or 3 (transfer lines WF1100 , WF1101, or WF1102 respectively) be used if there is a potential for the fluid to have a Bingham Plastic yield stress of larger than $1 \mathrm{~Pa}$ such as those of the 12,000L dissolver batch fluids. If transfer line WF1103 is used for such fluids, the fluid will backup in to the header in Building 221-H. The 10-inch header associated with Waste Header \#2 (WF1103) may backup and provide the necessary head for 25 gpm, but this was not analyzed.

The deposition velocity was calculated between 3.5 to $4.1 \mathrm{ft} / \mathrm{s}$. Given a discharge rate of $25 \mathrm{gpm}$, only the steepest transfer lines have a potential to mitigate settling of solids. Intermittent flushing with inhibited water is recommended in minimizing undissolved solids buildup at the maximum achievable flow rate with a minimum of at least 3 waste line volumes or an acceptable drop rate in the waste header liquid level is observed. 


\subsection{References}

1. (a) Parkins, W.E., Power Reactors - The Sodium Reactor Experiment, Proceedings of the International Conference on the Peaceful Uses of Atomic Energy, Geneva, United Nations - New York: Geneva, 1955, p 27. (b) Pickett, C. E., Recommended Disposition of SRE Fuel, CBU-HCP2004-00078, Savannah River Site, 2004, p 3.

2. Clifton, Jr., W. H., Flowsheet Evaluation for the Dissolving of Sodium Reactor Experiment Used Nuclear Fuel, TTR, NMMD-HTS-2012-3193, Savannah River Nuclear Solutions, Aiken, SC, January 30, 2012.

3. Rudisill, T. S.; Pierce, R. A., Dissolution of Plutonium Metal in 8-10 M Nitric Acid, SRNL-STI2012-00043, Rev. 0, Savannah River National Laboratory, Aiken, SC, 2012, p 58.

$4 \quad$ D129820, Bldg 221F/H Savannah River Plant Standard 6'- 0” DIA. X 6'-0” Cell Tank,

5 D127605, Bldg 221 F\&H, STIRRERS FOR 6'- 0” DIAMETER CELL TANK

6 W146044, SRS 200 AREA, BLDG 221 F/G, 3 H.P. AGITATOR ASSEMBLY FOR 6' - 0” DIA X 6' - 0" CELL TANK

7 Lambert, D. P., and al. et., "DETERMINING THE CAUSE FOR LOW FLOWRATES DURING AM?CM SIMULANT TESTING IN F AREA”, WSRC-TR-2002-00569, Rev. 0, April 29, 2003

8 Newell, J. D., and al. et., "Continuously Stirred Tank Reactor Parameters That Affect Sludge Batch 6 Simulant Properties”, SRNL-STI-2009-00603, May 2010

9 Koopman, D. C., and al. et., "RHEOLOGY IMPROVEMENTS DURING PREPARATION OF 40INCH HEEL CASE SIMULANTS FOR SLUDGE BATCH 4”, WSRC-STI-2006-00068, October 2006

10 Koopman, D. C., and al. et., "IMPACT OF PREPARATIN METHODS AND SCALE FACTORS ON SLUDGE BATCH 4 SIMULANT PROPERTIES”, WSRC-STI-2006-00088, November 2006

11. Haestad Methods, Chapter 1, "Basic Hydraulic Principles”, http://www.aces.edu/waterquality/streams/Fact\%20Sheets/Hydraulic\%20Basics.pdf, 2002.

12. Crane Technical Paper No. 410, "Flow of Fluids Through Valves, Fittings, and Pipe”, 1988.

13. Steimke, J. L., et al., "Engineering Development Laboratory (EDL) Draining Tests and Transfer Path Review”, SRT-WHM-2004-00017, Savannah River Laboratory, Aiken, SC, December 30, 2004.

14. Lauchlan, C. S., et al., “Air in Pipelines - A literature Review”, HR Wallingford, SR649, Rev. 2.0, April 2005.

15. Pozos, O., et al., “Air entrapped in gravity pipeline systems”, Journal of Hydraulic Research, Vol. 48, No. 3, pp. 338-347, 2010.

16. Mathworks, Inc., Natick, Massachusetts, Matlab Central File Exchange, http://www.mathworks.com/matlabcentral/fileexchange/7747.

17. Abulnaga, B., "Slurry Systems Handbook”, Chapter 6, McGraw-Hill, 2002.

18. Darby, R., "Chemical Engineering Fluids Mechanics”, Chapter 6, 2 ${ }^{\text {nd }}$, Mercel Dekker, 2001.

19. Turian, R. M., HSU, F. L., and Ma, T. W., "Estimation of the Critical Velocity in Pipeline Flow of Slurries”, Powder Technology, Volume 51, pp. 35-47, 1987.

20. Blevins, R. D., “Applied Fluid Dynamics Handbook”, “Chapter 8, Kreiger Publishing Company, 2003.

21. Yang, C. T., "Erosion and Sedimentation Manual”, Chapter 3 - Noncohesive Sediment Transport, U.S. Department of the Interior, Bureau of Reclamation, November 2006.

22. W715433, SRP BLDG 21H Replace Waste HDRS Transition Box Sections \& Details Process, Instruments \& Elect., Rev. 15.

23. W712040, SRP Bldg. 241H Replace Waste Header WF1100 \& WF1101 221-H-HPP5 \& HPP6, Piping Flexibility Analysis, Sheet 1, Rev. 7 
24. W712042, SRP Bldg. 241H Replace Waste Header WF1102 \& WF1103 221-H-HPP5 \& HPP6, Piping Flexibility Analysis, Sheet 3, Rev. 7.

25. W712648, SRP Bldg. 241H Replace Waste Header WF1102 \& WF1103 221-H-HPP5 \& HPP6, Piping Flexibility Analysis, Sheet 4, Rev. 2.

26. W712649, SRP Bldg. 241H Replace Waste Header WF1100 \& WF1101 221-H-HPP5 \& HPP6, Piping Flexibility Analysis, Sheet 2, Rev. 2.

27. D178553, Waste Line Details, Rev. 4.

28. D179291, Waste Line Details, Rev. 8.

29 Proctor, J.F., "SAVANNAH RIVER PLANT REPLACE WASTE HEADERS, WR 860604 BASIC DESIGN DATA”, 132220, October 3, 1978

30. S1-2-669, Jet Jumper.

31. Darby, R., “Chemical Engineering Fluid Mechanics”, Marcel Dekker, Inc., 2nd Edition, 2001.

32. Jessee, L., Cropping of HMI (BER-II) Fuel Assemblies (U), M-CLC-L-00285, Rev. 1, Savannah River Nuclear Solutions, Aiken, SC, September 28, 2009.

33. Laurinat, J. E., Calculation of Surface Area for Dissolution of MURR Fuel, X-CLC-H-00800, Rev. 0, Savannah River Nuclear Solutions, Aiken, SC, December 17, 2009.

34. Kyser, E. A., Dissolution of Irradiated MURR Fuel Assemblies, SRNL-STI-2010-00005, Revision 2, Savannah River National Lab, Aiken, SC, June 2010.

35. Karraker, D. G., Dissolution of Thorium in Mixtures of $\mathrm{HNO}_{3}$ and HF, DP-399, Savannah River Laboratory, Aiken, SC, 1959, p 15.

36. Blanco, R. E. Dissolution and Feed Adjustment, Symposium on the Reprocessing of Irradiated Fuels, Book 1, United States Atomic Energy Commission, Brussels, Belgium, May 20-25, 1957, p. 22-44.

37. Orth, D. A., SRP Thorium Processing Experience, DPSPU 78-30-3, American Nuclear Society Meeting, San Diego, CA, E. I. du Pont de Nemours \& Co., Savannah River Laboratory, Aiken, SC, June 18-23, 1978.

38. Clifton, Jr., W. H., KUR. RECH-I. FNR. and HOR UNF Campaign Validation, SRNS-EI1002010-00021, Rev. 0, Savannah River Nuclear Solutions, Aiken, SC, October 13, 2010.

39. Prout, W. E., Symonds, A. E., Recovery of Thorium and Uranium-233 from Irradiated Thorium Oxide and Metal, DP-1036, E. I. du Pont de Nemours \& Co., Savannah River Laboratory, Aiken, SC, January 1967.

40. Weitz, F. R. , H-Canyon Dissolver Hydrogen Dilution Calculations using Off-gas Specific Lower Flammability Limit (LFL), X-CLC-H-00473, Westinghouse Savannah River Company, Aiken, SC, October 2, 2003.

41. Scott, F. E. , Zabetakis, M. G., Flammability of Hydrogen-Air-Nitrogen Oxide Mixtures, AECU3178 or BM-3507, United States Department of the Interior; Bureau of Mines, Pittsburg, PA, 1956.

42. Dyer, W. G., Williams, J. C., Impact of Temperature on Hydrogen Lower Flammability Limit for Separations, Facilities, WSRC-TR-2003-00313, Rev 0, Westinghouse Savannah River Company, Aiken, SC, July 2003.

43. Caracciolo, V. P., Dissolver for Uranium-Aluminum Alloy Tubes, DP-398, E. I. du Pont de Nemours and Company, Savannah River Laboratory, Aiken, SC, September 1959.

44. Moore, R. L., Goodall, C. A., Hepworth, J. L., Watts, Jr., R. A., Nitric Acid Dissolution of Thorium, Hanford Atomic Products Operation, General Electric Co., Richland, Wash. May 1957.

45 A. S. Russell, J. D. Edwards, and C. S. Taylor, "Solubility and Density of Hydrated Aluminas in NaOH Solutions”, J. of Metals, 1955, 1123-1128.

46. Perkins, W. C., Dissolving Uranium Oxide Fuel Aluminum, DP-1337, E. I. du Pont de Nemours and Company, Savannah River Laboratory, Aiken, SC, Nov. 1973. 
SRNL-STI-2012-00279

Revision 1

Appendix A. Surface Area Calculations 


\section{A.1. $\quad$ Disassembly L-Area Bundling Tube Surface Area Calculations}

The surface area per unit height of the L-Bundle as described in the SRNS drawing C-CS-L-0962 revision 7 was derived. A sketch of the L-Bundle is shown in Figure A-1. The various parts of the L-Bundle have been color coded and the dimensions of these parts are shown in Table A-1.

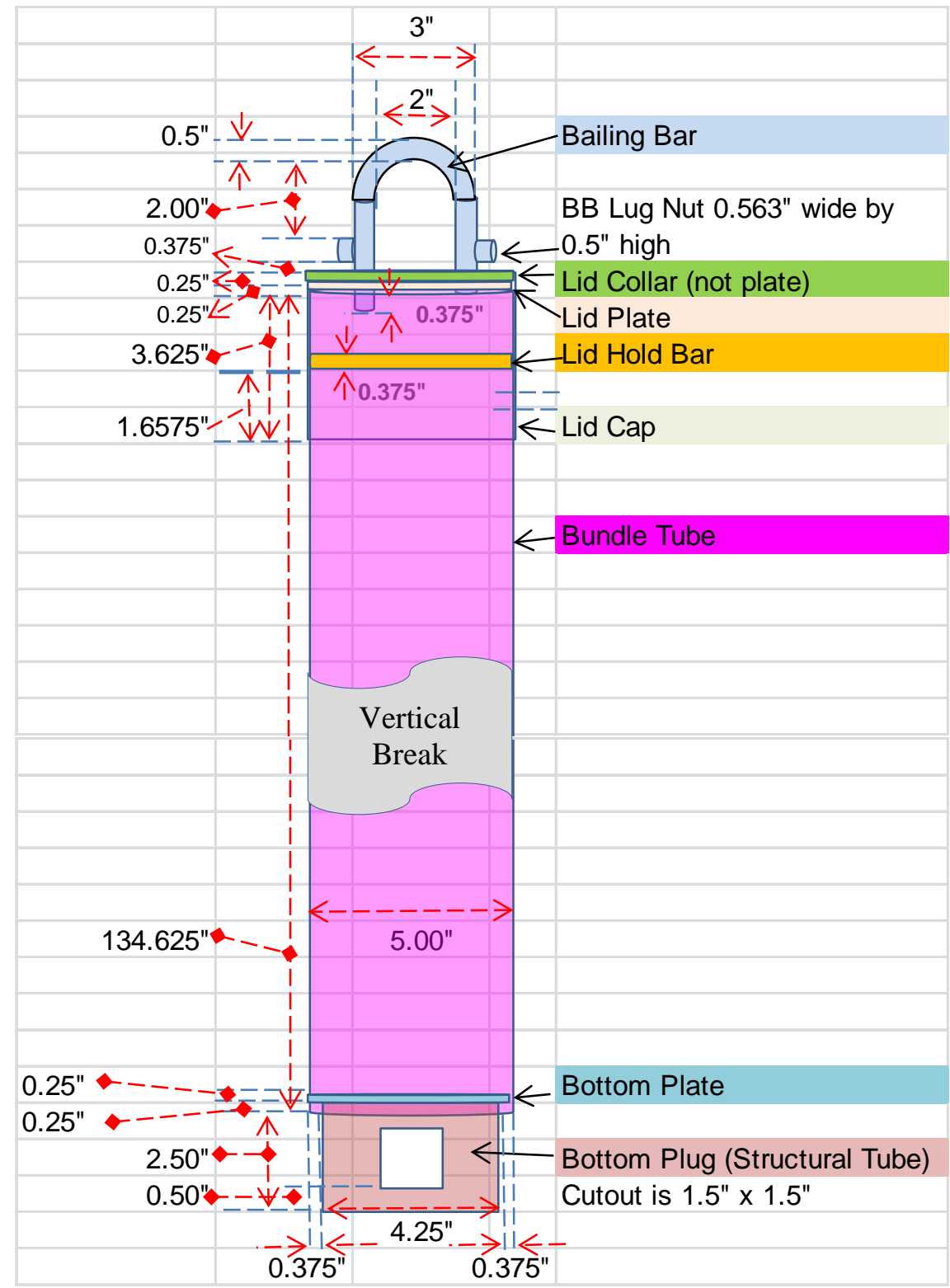

Figure A-1. Sketch of Disassembly L-Area Bundling Tube (DABT) or L-Bundle 
SRNL-STI-2012-00279

Revision 1

Table A-1. Disassembly L-Area Bundling Tube Parts and Dimensions

\begin{tabular}{|c|c|c|c|c|}
\hline L-Bundle Part & $\begin{array}{l}\text { Outer } \\
\text { Diameter } \\
\text { (OD) [in] }\end{array}$ & $\begin{array}{l}\text { Wall } \\
\text { [in] }\end{array}$ & $\begin{array}{l}\text { Length } \\
\text { (vertical } \\
\text { height) [in] }\end{array}$ & Comments \\
\hline Bailing Bar & 0.500 & $0.500 *$ & 8.552 & $\begin{array}{l}\text { Believe correct bail bar length as } 1.875 \text { in. slot in } \\
\text { bundle tube for holding bar and treat holding bar } \\
\text { separate. }\end{array}$ \\
\hline Lid Collar & 5.080 & 0.052 & 0.250 & $\begin{array}{l}\text { Since calls collar treat as annular ring on top of } \\
\text { lid plate. }\end{array}$ \\
\hline Lid Plate & 4.976 & NA & 0.250 & $\begin{array}{l}\text { Has eight equally space } 0.25 \text { in. OD holes plus } \\
\text { two } 0.5 \text { in. OD holes for bail bar. }\end{array}$ \\
\hline Lid Hold Bar & 0.375 & NA & 5.000 & $\begin{array}{l}\text { Welded to lid to go into } 1.875 \text { in. slot on bundle } \\
\text { tube. }\end{array}$ \\
\hline Lid Cap & 5.080 & 0.052 & 4.125 & $\begin{array}{l}\text { Note length (or height) includes } 0.25 \text { in. tall lid } \\
\text { plate and } 0.25 \text { in. tall collar. }\end{array}$ \\
\hline Bundle Tube & 5.000 & 0.052 & 134.625 & None \\
\hline Bottom Plate & 4.896 & NA & 0.250 & $\begin{array}{l}\text { Has } 8 \text { equally space } 0.25 \text { in. OD holes. Note that } \\
\text { bottom plate sits } 0.25 \text { in up from bottom of } \\
\text { bundle tube. }\end{array}$ \\
\hline Bottom Plug (Structural Tube) & 4.250 & 0.250 & 2.750 & $\begin{array}{c}\text { Two } 180^{\circ} \text { apart } 1.5 \text { in. x } 1.5 \text { in. slots in Bottom } \\
\text { Plug } 0.5 \text { in. off bottom. Bottom Plug recessed } \\
0.25 \text { in. up into Bundle Tube where contacts } \\
\text { Bottom Plate giving exposed } 2.5 \text { in. below } \\
\text { bundle tube }\end{array}$ \\
\hline
\end{tabular}

NA=Not Applicable, $*$ Solid Thickness

Starting from the bottom of the L-Bundle and going up vertically the exposed inner and outer surface area of L-Bundle is calculated as shown in Table A-2. This table details the surface area calculations for specific sections of the L-Bundle in terms of height from its bottom. The first column is called the Scaling Factor for Height $h$ or the area per unit height for each section of the L-Bundle. The second column is the Height $h$ of each section of the L-Bundle. The third column is the Constant area term for each section of the L-Bundle like the previous section's surface area or a specific surface area at the boundary between sections. The fourth column is the Cumulative Surface Area term which is the total area up to the specific height of each section which is calculated as the scaling factor for height times height $h$ plus the constant. The fifth and sixth columns are the from and to height respectively from the bottom of the L-Bundle. These from and to columns can be used to find the specific surface area at a specific height if desired. The seventh column is the Description of the particular section of the L-Bundle where the surface area calculation applies. The eighth column is Surface Area Sums in the particular section of the L-Bundle. The [ ] in the Area Sums reflect calculation values used in the Scaling Factor for Height $h$ and Constant columns as shown by the formulae where h terms go into the Scaling Factor for Height $h$ and Constant terms go into the Constant term. Using the logic outlined here, the total inner and outer surface area for the L-Bundle for the full 141 inch height was 4335.83 in $^{2}$ giving an overall scaling factor for the L-Bundle of $30.75 \mathrm{in}^{2} /$ in or $2.56 \mathrm{ft}^{2} / \mathrm{ft}$. 
Table A-2. Inner and Outer Surface Area Calculation for DABT or L-Bundle

\begin{tabular}{|c|c|c|c|c|c|c|c|}
\hline \begin{tabular}{|c|} 
Scaling \\
Factor for \\
Height h \\
{$\left[\right.$ in $^{2} /$ in] } \\
\end{tabular} & \begin{tabular}{|c|} 
Height h \\
[in]
\end{tabular} & $\begin{array}{c}\text { Constant } \\
{\left[\text { in }^{2}\right]}\end{array}$ & $\begin{array}{c}\text { Cumulative } \\
\text { Surface } \\
\text { Area }\left[i^{2}\right] \\
\end{array}$ & $\begin{array}{c}\text { From } \\
\text { [in] }\end{array}$ & To [in] & Description & Surface Area Sums \\
\hline 25.13 & 0.50 & 3.14 & 15.71 & 0 & 0.5 & $\begin{array}{l}\text { from bottom of structural } \\
\text { tube (bottom plug or BP) } \\
\text { up to start of } 1.5 " \text { cutouts }\end{array}$ & $\begin{array}{l}\text { [Bottom Annular Ring of BP }]+[\text { Outside Circumference Area of } \\
\text { BP }]+[\text { Inside Circumference Area of BP }]=\left[\pi / 4 *\left(4.25^{2}-3.75^{2}\right)\right] \\
+\left[\pi^{*} 4.25^{*} \mathrm{~h}\right]+\left[\pi^{*} 3.75 * \mathrm{~h}\right]\end{array}$ \\
\hline 23.63 & 1.50 & 15.71 & 51.16 & 0.5 & 2 & $\begin{array}{l}\text { from bottom of 1.5" } \\
\text { cutouts on bottom plug up } \\
\text { to top of 1.5" cutouts on } \\
\text { BP }\end{array}$ & $\begin{array}{c}\text { [Outside Circumference Area of BP] }+ \text { [Inside Circumference } \\
\text { Area of BP] - [Outside Face Area of Cutouts }-[\text { Inside Face Area } \\
\text { of Cutouts }]+[\text { Inside Edge Area of Cutouts }]=\left[\pi^{*} 4.25^{*} \mathrm{~h}\right]+ \\
{\left[\pi^{*} 3.75^{*} \mathrm{~h}\right]-\left[1.5^{*} \mathrm{~h}\right]-\left[1.5^{*} \mathrm{~h}\right]+\left[2 *\left(1.5^{*} 0.25+\mathrm{h} * 0.25^{*} 2\right)\right]}\end{array}$ \\
\hline 25.13 & 0.50 & 51.91 & 64.47 & 2 & 2.5 & $\begin{array}{l}\text { from top of } 1.5 " \text { cutouts } \\
\text { on BP up to bottom of } \\
\text { Bundle Tube }\end{array}$ & $\begin{array}{c}\text { [Outside Circumference Area of BP] }+ \text { [Inside Circumference } \\
\text { Area of BP }]+[\text { Top Inside Edge Area of Cutouts }]=\left[\pi^{*} 4.25^{*} \mathrm{~h}\right]+ \\
{\left[\pi^{*} 3.75^{*} \mathrm{~h}\right]+\left[2 *\left(1.5^{*} 0.25\right)\right]}\end{array}$ \\
\hline 29.06 & 0.25 & 65.28 & 72.55 & 2.5 & 2.75 & $\begin{array}{l}\text { from bottom of Bundle } \\
\text { Tube up to bottom of } \\
\text { bottom plate }\end{array}$ & $\begin{array}{c}\text { [Outside Circumference area of Bundle Tube }]+[\text { Inside } \\
\text { Circumference area of BP] }+[\text { bottom annular ring of Bundle } \\
\text { Tube }]=[\pi * 5 * \mathrm{~h}]+[\pi * 4.25 * \mathrm{~h}]+\left[\pi / 4 *\left(5^{2}-4.896^{2}\right)\right]\end{array}$ \\
\hline 21.99 & 0.25 & 87.84 & 93.34 & 2.75 & 3 & $\begin{array}{l}\text { from bottom of bottom } \\
\text { plate up to top of bottom } \\
\text { plate }\end{array}$ & $\begin{array}{l}\text { [Inside Surface area of } 0.25 " \text { holes] }+ \text { [Outside Circumference } \\
\text { area of Bundle Tube] }+ \text { [bottom surface area of bottom plate] }= \\
{[8 *(\pi * 0.25 * \mathrm{~h})]+\left[\pi^{*} 5 * \mathrm{~h}\right]+\left[\pi / 4 * 4.896^{2}-8 * \pi / 4 * 0.25^{2}-\right.} \\
\left.\pi / 4 *\left(4.25^{2}-3.75^{2}\right)\right]\end{array}$ \\
\hline 31.09 & 130.50 & 111.77 & 4168.91 & 3 & 133.5 & $\begin{array}{l}\text { from top of bottom plate } \\
\text { up to bottom of lid where } \\
\text { overlaps Bundle Tube }\end{array}$ & $\begin{array}{l}\text { [Inside Circumference area of Bundle Tube] }+ \text { [Outside } \\
\text { Circumference area of Bundle Tube] }+ \text { [top surface area of } \\
\text { bottom plate }]=[\pi * 4.896 * h]+[\pi * 5 * h]+\left[\pi / 4 * 4.896^{2}-\right. \\
\left.\qquad 8 * \pi / 4 * 0.25^{2}\right]\end{array}$ \\
\hline 31.34 & 1.66 & 4168.91 & 4220.86 & 133.5 & 135.16 & $\begin{array}{l}\text { from bottom of lid where } \\
\text { overlaps Bundle Tube up } \\
\text { to bottom of holding bar }\end{array}$ & $\begin{array}{c}\text { [Outside Circumference area of Lid] }+ \text { [Inside Circumference } \\
\text { area of Bundle Tube }]=\left[\pi^{*} 5.08 * \mathrm{~h}\right]+\left[\pi^{*} 4.896 * \mathrm{~h}\right]\end{array}$ \\
\hline 51.36 & 0.19 & 4220.86 & 4230.49 & 135.16 & 135.35 & $\begin{array}{l}\text { from bottom of holding } \\
\text { bar up to middle of } \\
\text { holding bar }\end{array}$ & $\begin{array}{c}\text { [Outside Circumference area of Lid] + [Inside Circumference } \\
\text { area of Bundle Tube] }+ \text { [circumference area of holding bar] - [end } \\
\text { face areas of holding bar against lid] }=[\pi * 5.08 * \mathrm{~h}]+\left[\pi * 4.896^{*} \mathrm{~h}\right] \\
+\left[2 * 5 * 0.375^{*} \cos ^{-1}(1-8 / 3 * \mathrm{~h})\right]-\left[2 * 0.375^{*}\left\{0.375^{*} \cos ^{-1}(1-\right.\right. \\
\left.\left.8 / 3 * \mathrm{~h})-(0.375-\mathrm{h}) * \sin \left(\cos ^{-1}(1-8 / 3 * \mathrm{~h})\right)\right\}\right]\end{array}$ \\
\hline
\end{tabular}


Table A-2. Inner and Outer Surface Area Calculation for DABT or L-Bundle

\begin{tabular}{|c|c|c|c|c|c|c|c|}
\hline \begin{tabular}{|c|} 
Scaling \\
Factor for \\
Height h \\
{$\left[\right.$ in $^{2} /$ in] $]$} \\
\end{tabular} & \begin{tabular}{|c|} 
Height h \\
[in]
\end{tabular} & $\begin{array}{c}\text { Constant } \\
{\left[\text { in }^{2}\right]}\end{array}$ & $\begin{array}{l}\text { Cumulative } \\
\text { Surface } \\
\text { Area }\left[i^{2}\right] \\
\end{array}$ & $\begin{array}{c}\text { From } \\
\text { [in] }\end{array}$ & To [in] & Description & Surface Area Sums \\
\hline 41.29 & 0.19 & 4230.49 & 4238.23 & 135.35 & 135.53 & $\begin{array}{l}\text { from middle of holding } \\
\text { bar up to top of holding } \\
\text { bar }\end{array}$ & $\begin{array}{c}\text { [Outside Circumference area of Lid] }+ \text { [Inside Circumference } \\
\text { area of Bundle Tube] }+ \text { [circumference area of holding bar] - [end } \\
\text { face areas of holding bar against lid] }=\left[\pi * 5.08^{*} \mathrm{~h}\right]+\left[\pi^{*} 4.896^{*} \mathrm{~h}\right] \\
+\left[2 * 5 * 0.375^{*}\left(\pi / 2-\cos ^{-1}(\mathrm{~h} / 0.375)\right)\right]-\left[1 / 2 * 0.375 * 0.375^{*}(\pi / 2-\right. \\
\left.\left.\cos ^{-1}(\mathrm{~h} / 0.375)\right)+\mathrm{h} * \sqrt{0.375^{2}-\mathrm{h}^{2}}\right]\end{array}$ \\
\hline 31.34 & 1.21 & 4238.23 & 4276.23 & 135.53 & 136.745 & $\begin{array}{c}\text { from top of holding bar up } \\
\text { to bottom of bail bar in } \\
\text { bundle tube }\end{array}$ & $\begin{array}{c}\text { [Outside Circumference area of Lid }]+[\text { Inside Circumference } \\
\text { area of Bundle Tube }]=[\pi * 5.08 * \mathrm{~h}]+\left[\pi^{*} 4.896 * \mathrm{~h}\right]\end{array}$ \\
\hline 32.91 & 0.38 & 4276.43 & 4288.77 & 136.75 & 137.12 & $\begin{array}{l}\text { from bottom of bail bar in } \\
\text { bundle tube up to bottom } \\
\text { of lid plate }\end{array}$ & $\begin{array}{c}\text { [Outside Circumference area of Lid }]+[\text { Inside Circumference } \\
\text { area of Bundle Tube }]+[\text { circumference area of bail bar }]+[\text { end } \\
\text { face area of bail bar }]=\left[\pi^{*} 5.08 * \mathrm{~h}\right]+\left[\pi^{*} 4.896 * \mathrm{~h}\right]+\left[\pi^{*} 0.5 * \mathrm{~h}\right]+ \\
{\left[\pi^{*}(0.5 / 2)^{2}\right]}\end{array}$ \\
\hline 22.24 & 0.25 & 4307.82 & 4313.38 & 137.12 & 137.37 & $\begin{array}{l}\text { from bottom of lid plate } \\
\text { up to top of lid plate (don't } \\
\text { count bail bar holes since } \\
\text { bail bar fills) }\end{array}$ & $\begin{array}{c}\text { [inside surface area of } 0.25 " \text { holes }]+[\text { Outside Circumference } \\
\text { area of Lid] }+[\text { bottom surface area of lid plate }]=\left[8^{*} \pi^{*} 0.25^{*} \mathrm{~h}\right]+ \\
{\left[\pi^{*} 5.08 * \mathrm{~h}\right]+\left[\pi^{*}(4.976 / 2)^{2}-8^{*} \pi^{*}(0.25 / 2)^{2}\right]}\end{array}$ \\
\hline 34.73 & 0.25 & 4313.38 & 4322.07 & 137.37 & 137.62 & $\begin{array}{l}\text { from top of lid plate (don't } \\
\text { count bail bar holes since } \\
\text { bail bar fills) up to top of } \\
0.052 \text { thick lid collar }\end{array}$ & $\begin{array}{c}\text { [inside Circumference area of lid collar }]+[\text { Outside } \\
\text { Circumference area of lid collar }]+[\text { Circumference area of bail } \\
\text { bar }]=\left[\pi^{*} 4.976 * \mathrm{~h}\right]+\left[\pi^{*} 5.08 * \mathrm{~h}\right]+\left[2 * \pi^{*} 0.5 * \mathrm{~h}\right]\end{array}$ \\
\hline 3.14 & 0.38 & 4322.89 & 4324.07 & 137.62 & 138 & $\begin{array}{c}\text { from top of } 0.052 " \text { thick } \\
\text { lid collar to bottom of bail } \\
\text { bar lugs }\end{array}$ & $\begin{array}{l}\text { [circumference area of bail bar] }+ \text { [top annular ring SA of lid } \\
\text { collar }]=\left[2 * \pi^{*} 0.5^{*} \mathrm{~h}\right]+\left[\pi^{*}(5.08 / 2)^{2}-\pi^{*}(4.976 / 2)^{2}\right]\end{array}$ \\
\hline 4.91 & 0.25 & 4324.07 & 4325.29 & 138 & 138.25 & $\begin{array}{l}\text { from bottom of bail bar } \\
\text { lugs up to middle of bail } \\
\text { bar lugs }\end{array}$ & $\begin{array}{c}\text { [circumference area of bail bar] }+[\text { circumference area of lug] } \\
\text { (note [end area of lug exposed] - [end area of lug against bail bar] } \\
\text { cancel one another and circumference area of bail bar covered by } \\
\text { lug is greater than the end area of lug exposed) }=\left[2 * \pi^{*} 0.5^{*} \mathrm{~h}\right]+ \\
{\left[0.5^{*} 0.563^{*} \cos ^{-1}(1-\mathrm{h} / 0.25)\right]}\end{array}$ \\
\hline
\end{tabular}


Table A-2. Inner and Outer Surface Area Calculation for DABT or L-Bundle

\begin{tabular}{|c|c|c|c|c|c|c|c|}
\hline \begin{tabular}{|c|} 
Scaling \\
Factor for \\
Height h \\
{$\left[\right.$ in $^{2} /$ in] $]$} \\
\end{tabular} & \begin{tabular}{|c|} 
Height h \\
[in]
\end{tabular} & $\begin{array}{c}\text { Constant } \\
{\left[\text { in }^{2}\right]}\end{array}$ & $\begin{array}{l}\text { Cumulative } \\
\text { Surface } \\
\text { Area }\left[i^{2}\right] \\
\end{array}$ & $\begin{array}{c}\text { From } \\
\text { [in] }\end{array}$ & To [in] & Description & Surface Area Sums \\
\hline 4.91 & 0.25 & 4325.29 & 4326.52 & 138.25 & 138.5 & $\begin{array}{l}\text { from middle of bail bar } \\
\text { lugs up to top of bail bar } \\
\text { lugs }\end{array}$ & $\begin{array}{c}\text { [circumference area of bail bar] }+ \text { [circumference area of lug] } \\
\text { (note [end area of lug exposed] - [end area of lug against bail bar] } \\
\text { cancel one another and circumference area of bail bar covered by } \\
\text { lug is greater than end area of lug exposed) }=\left[2 * \pi^{*} 0.5^{*} \mathrm{~h}\right]+ \\
{\left[0.563 *\left(\pi / 2-\cos ^{-1}(\mathrm{~h} / 0.25)\right) * 0.5\right]}\end{array}$ \\
\hline 3.14 & 1.00 & 4326.52 & 4329.66 & 138.5 & 139.5 & $\begin{array}{l}\text { from top of bail bar lugs } \\
\text { up to start of arc on bail } \\
\text { bar }\end{array}$ & {$[$ circumference area of bail bar $]=\left[2 * \pi^{*} 0.5^{*} \mathrm{~h}\right]$} \\
\hline 4.11 & 1.50 & 4329.66 & 4335.83 & 139.5 & 141 & $\begin{array}{l}\text { from start of arc on bail } \\
\text { bar up to top of bail bar }\end{array}$ & $\begin{array}{l}\text { [fraction of bail bar arc SA covered] (note treat circumference } \\
\text { area of arc as } 2 \text { straight pieces each with half length of } 180^{\circ} \text { arc } \\
\left.\text { with diameter of } 2.5^{\prime \prime}\right)=\left[\mathrm{h} / 1.5^{*} \pi / 2^{*} 2.5^{*} \pi^{*} 0.5\right]\end{array}$ \\
\hline
\end{tabular}


In a prior set of dissolution calculations for the MURR assemblies, only the external area of L-Bundle was used. For the SRE, HMI, and DR-3 assemblies documented in this report, the predicted clearances between the outermost dimension of these assemblies and the inner diameter of the L-Bundle (4.896 in.) are shown in Table A-3. Based on the MURR assemblies dissolution calculations ${ }^{34}$ and its references, any surfaces separated by 2 to $9.7 \mathrm{~mm}$ are considered too close to be included in the surface area calculations due to the gas layer forming on the "close" surfaces. Based on the clearances described in Table A-3 and the general shape of the assemblies, it was decided to ignore the internal surface parts of the L-Bundle so a set of calculations were performed where only the outer surface area parts of the LBundle were counted and the internal surface area parts were not counted but shown as strike through in Table A-4 for completeness. Using the logic outlined here, the total outer surface area for the L-Bundle for the full 141 inch height was $2236.74 \mathrm{in}^{2}$ giving an overall scaling factor for the L-Bundle of 15.86 in $^{2} /$ in or $1.32 \mathrm{ft}^{2} / \mathrm{ft}$.

Table A-3. Clearances between L-Bundle and SRE, HMI, and DR-3 Assemblies

\begin{tabular}{|c|c|c|c|}
\hline Assembly & $\begin{array}{c}\text { Assembly } \\
\text { Outermost } \\
\text { Dimension }\end{array}$ & $\begin{array}{c}\text { Clearance between Bundling Tube } \\
\text { Inner Wall and Assembly Outermost } \\
\text { Dimension }\end{array}$ \\
\hline SRE & {$[\mathrm{in}]$} & {$[\mathrm{in}]$} & {$[\mathrm{mm}]$} \\
\hline HMI & 3.50 & 0.698 & 17.7 \\
\hline DR-3 & 3.37 & 0.763 & 19.4 \\
\hline
\end{tabular}


Table A-4. Outer Surface Area Calculation for DABT or L-Bundle

\begin{tabular}{|c|c|c|c|c|c|c|c|}
\hline \begin{tabular}{|c|} 
Scaling \\
Factor for \\
Height h \\
{$\left[\right.$ in $^{2} /$ in] }
\end{tabular} & $\begin{array}{l}\text { Height h } \\
\text { [in] }\end{array}$ & $\begin{array}{c}\text { Constant } \\
{\left[\text { in }^{2}\right]}\end{array}$ & $\begin{array}{c}\text { Cumulative } \\
\text { Surface } \\
\text { Area }\left[i^{2}\right]\end{array}$ & $\begin{array}{c}\text { From } \\
\text { [in] }\end{array}$ & To [in] & Description & Surface Area Sums \\
\hline 13.35 & 0.50 & 3.14 & 9.82 & 0.00 & 0.50 & $\begin{array}{l}\text { from bottom of structural } \\
\text { tube (bottom plug or BP) } \\
\text { up to start of } 1.5^{\prime \prime} \text { cutouts }\end{array}$ & $\begin{array}{c}\text { [Bottom Annular Ring of BP }]+[\text { Outside Circumference Area of } \\
\text { BP }]+ \text { [nside Cireumference Area of BP }]=\left[\pi / 4 *\left(4.25^{2}-3.75^{2}\right)\right] \\
+[\pi * 4.25 * h]+[\pi * 3.75 * h]\end{array}$ \\
\hline 13.35 & 1.50 & 9.82 & 29.85 & 0.50 & 2.00 & $\begin{array}{l}\text { from bottom of } 1.5^{\prime \prime} \\
\text { cutouts on bottom plug up } \\
\text { to top of } 1.5^{\prime \prime} \text { cutouts on } \\
\text { BP }\end{array}$ & $\begin{array}{c}\text { [Outside Circumference Area of BP] }+ \text { [nside Circumference } \\
\text { Area of BP] - [Outside Face Area of Cutouts] - Inside Face Area } \\
\text { efCutouts] }+[\text { Inside Edge Area of Cutouts }]=\left[\pi^{*} 4.25^{*} \mathrm{~h}\right]+ \\
{\left[\pi^{*} 3.75 * \mathrm{~h}\right]-[1.5 * \mathrm{~h}]-[1.5 * \mathrm{~h}]+\left[2 *\left(1.5 * 0.25+\mathrm{h} * 0.25^{*} 2\right)\right]}\end{array}$ \\
\hline 13.35 & 0.50 & 30.60 & 37.27 & 2.00 & 2.50 & $\begin{array}{l}\text { from top of } 1.5^{\prime \prime} \text { cutouts } \\
\text { on BP up to bottom of } \\
\text { Bundle Tube }\end{array}$ & $\begin{array}{c}\text { [Outside Circumference Area of BP }]+ \text { [nside Circumference } \\
\text { Area of BP }]+[\text { Top Inside Edge Area of Cutouts }]=\left[\pi^{*} 4.25 * \mathrm{~h}\right]+ \\
{\left[\pi^{*} 3.75^{*} \mathrm{~h}\right]+\left[2 *\left(1.5^{*} 0.25\right)\right]}\end{array}$ \\
\hline 15.71 & 0.25 & 38.08 & 42.01 & 2.50 & 2.75 & $\begin{array}{l}\text { from bottom of Bundle } \\
\text { Tube up to bottom of } \\
\text { bottom plate }\end{array}$ & $\begin{array}{l}\text { [Outside Circumference area of Bundle Tube }]+ \text { Enside } \\
\text { Eiremmference area of BP }]+[\text { bottom annular ring of Bundle } \\
\text { Tube }]=[\pi * 5 * h]+[\pi * 4.25 * \mathrm{~h}]+\left[\pi / 4 *\left(5^{2}-4.896^{2}\right)\right]\end{array}$ \\
\hline 21.99 & 0.25 & 57.30 & 62.80 & 2.75 & 3.00 & $\begin{array}{l}\text { from bottom of bottom } \\
\text { plate up to top of bottom } \\
\text { plate }\end{array}$ & $\begin{array}{c}\text { [Inside Surface area of } 0.25 " \text { holes }]+[\text { Outside Circumference } \\
\text { area of Bundle Tube] }+[\text { bottom surface area of bottom plate }]= \\
{\left[8^{*}\left(\pi^{*} 0.25^{*} \mathrm{~h}\right)\right]+\left[\pi^{*} 5 * \mathrm{~h}\right]+\left[\pi / 4 *\left(4.896^{2}-8 * \pi / 4 * 0.25^{2}-\right.\right.} \\
\left.\pi / 4 *\left(4.25^{2}-3.75^{2}\right)\right]\end{array}$ \\
\hline 15.71 & 130.50 & 81.23 & 2131.12 & 3.00 & 133.50 & $\begin{array}{l}\text { from top of bottom plate } \\
\text { up to bottom of lid where } \\
\text { overlaps Bundle Tube }\end{array}$ & $\begin{array}{c}\text { Inside Circumference area of Bundle Tube }]+[\text { Outside } \\
\text { Circumference area of Bundle Tube] }+[\text { top surface area of } \\
\text { bottom plate }]=[\pi * 4.896 * h]+[\pi * 5 * h]+\left[\pi / 4 * 4.896^{2}-\right. \\
\left.8 * \pi / 4 * 0.25^{2}\right]\end{array}$ \\
\hline 15.96 & 1.66 & 2131.12 & 2157.57 & 133.50 & 135.16 & $\begin{array}{l}\text { from bottom of lid where } \\
\text { overlaps Bundle Tube up } \\
\text { to bottom of holding bar }\end{array}$ & $\begin{array}{c}\text { [Outside Circumference area of Lid] }+ \text { [nside Circumference } \\
\text { area of Bundle Tube }]=\left[\pi^{*} 5.08 * \mathrm{~h}\right]+\left[\pi^{*} 4.896 * \mathrm{~h}\right]\end{array}$ \\
\hline 15.96 & 0.19 & 2157.57 & 2160.56 & 135.16 & 135.35 & $\begin{array}{l}\text { from bottom of holding } \\
\text { bar up to middle of } \\
\text { holding bar }\end{array}$ & $\begin{array}{l}\text { [Outside Circumference area of Lid] + [Inside Circumference } \\
\text { area of Bundle Tube] + [circumference area of holding bar] - [end } \\
\text { face areas of holding bar against lid }]=[\pi * 5.08 * \mathrm{~h}]+[\pi * 4.896 * \mathrm{~h}] \\
+\left[2 * 5 * 0.375 * \cos ^{-1}(1-8 / 3 * \mathrm{~h})\right]-\left[2 * 0.375 *\left\{0.375^{*} \cos ^{-1}(1-\right.\right. \\
\left.\left.8 / 3 * \mathrm{~h})-(0.375-\mathrm{h}) * \sin \left(\cos ^{-1}(1-8 / 3 * \mathrm{~h})\right)\right\}\right]\end{array}$ \\
\hline
\end{tabular}


Table A-4. Outer Surface Area Calculation for DABT or L-Bundle

\begin{tabular}{|c|c|c|c|c|c|c|c|}
\hline \begin{tabular}{|c|} 
Scaling \\
Factor for \\
Height h \\
{$\left[\right.$ in $^{2} /$ in] }
\end{tabular} & $\begin{array}{l}\text { Height h } \\
{[\text { [in] }}\end{array}$ & $\begin{array}{c}\text { Constant } \\
{\left[\text { in }^{2}\right]}\end{array}$ & $\begin{array}{c}\text { Cumulative } \\
\text { Surface } \\
\text { Area }\left[i^{2}\right]\end{array}$ & $\begin{array}{c}\text { From } \\
\text { [in] }\end{array}$ & To [in] & Description & Surface Area Sums \\
\hline 15.96 & 0.19 & 2160.56 & 2163.56 & 135.35 & 135.53 & $\begin{array}{l}\text { from middle of holding } \\
\text { bar up to top of holding } \\
\text { bar }\end{array}$ & $\begin{array}{c}\text { [Outside Circumference area of Lid] + [Inside Circumference } \\
\text { area of Bundle Tube] + [ circumference area of holding bar] - [end } \\
\text { face areas of holding bar against lid] }=[\pi * 5.08 * \mathrm{~h}]+[\pi * 4.896 * \mathrm{~h}] \\
\neq\left[2 * 5 * 0.375 *\left(\pi / 2-\cos ^{-1}(\mathrm{~h} / 0.375)\right)\right]\left[1 / 2^{*} 0.375 * 0.375 *(\pi / 2\right. \\
\left.\left.\cos ^{-1}(\mathrm{~h} / 0.375)\right)+\mathrm{h} * \sqrt{0.375^{2}-\mathrm{h}^{2}}\right]\end{array}$ \\
\hline 15.96 & 1.21 & 2163.56 & 2182.91 & 135.53 & 136.75 & $\begin{array}{c}\text { from top of holding bar up } \\
\text { to bottom of bail bar in } \\
\text { bundle tube }\end{array}$ & $\begin{array}{c}\text { [Outside Circumference area of Lid }]+[\text { Inside Circumference } \\
\text { area of Bundle Tube }]=[\pi * 5.08 * \mathrm{~h}]+[\pi * 4.896 * \mathrm{~h}]\end{array}$ \\
\hline 17.53 & 0.38 & 2183.10 & 2189.68 & 136.75 & 137.12 & $\begin{array}{l}\text { from bottom of bail bar in } \\
\text { bundle tube up to bottom } \\
\text { of lid plate }\end{array}$ & $\begin{array}{c}\text { [Outside Circumference area of Lid }]+ \text { Inside Cireumference } \\
\text { area of Bundle Tube }]+[\text { circumference area of bail bar }]+[\text { end } \\
\text { face area of bail bar }]=[\pi * 5.08 * h]+[\pi * 4.896 * h]+[\pi * 0.5 * h]+ \\
{\left[\pi^{*}(0.5 / 2)^{2}\right]}\end{array}$ \\
\hline 22.24 & 0.25 & 2208.73 & 2214.29 & 137.12 & 137.37 & $\begin{array}{l}\text { from bottom of lid plate } \\
\text { up to top of lid plate (don't } \\
\text { count bail bar holes since } \\
\text { bail bar fills) }\end{array}$ & $\begin{array}{c}\left.\text { [inside surface area of } 0.25^{\prime} \text { holes }\right]+[\text { Outside Circumference } \\
\text { area of Lid] }+[\text { bottom surface area of lid plate }]=\left[8^{*} \pi^{*} 0.25^{*} \mathrm{~h}\right]+ \\
{\left[\pi^{*} 5.08^{*} \mathrm{~h}\right]+\left[\pi^{*}(4.976 / 2)^{2}-8^{*} \pi^{*}(0.25 / 2)^{2}\right]}\end{array}$ \\
\hline 34.73 & 0.25 & 2214.29 & 2222.98 & 137.37 & 137.62 & $\begin{array}{l}\text { from top of lid plate (don't } \\
\text { count bail bar holes since } \\
\text { bail bar fills) up to top of } \\
0.052 \text { thick lid collar }\end{array}$ & $\begin{array}{c}\text { [inside Circumference area of lid collar }]+[\text { Outside } \\
\text { Circumference area of lid collar }]+[\text { Circumference area of bail } \\
\text { bar }]=\left[\pi^{*} 4.976^{*} \mathrm{~h}\right]+\left[\pi^{*} 5.08^{*} \mathrm{~h}\right]+\left[2 * \pi^{*} 0.5^{*} \mathrm{~h}\right]\end{array}$ \\
\hline 3.14 & 0.38 & 2223.80 & 2224.98 & 137.62 & 138.00 & $\begin{array}{c}\text { from top of } 0.052 " \text { thick } \\
\text { lid collar to bottom of bail } \\
\text { bar lugs }\end{array}$ & $\begin{array}{l}\text { [circumference area of bail bar] }+ \text { [top annular ring SA of lid } \\
\text { collar }]=\left[2^{*} \pi^{*} 0.5^{*} \mathrm{~h}\right]+\left[\pi^{*}(5.08 / 2)^{2}-\pi^{*}(4.976 / 2)^{2}\right]\end{array}$ \\
\hline 4.91 & 0.25 & 2224.98 & 2226.20 & 138.00 & 138.25 & $\begin{array}{l}\text { from bottom of bail bar } \\
\text { lugs up to middle of bail } \\
\text { bar lugs }\end{array}$ & $\begin{array}{c}\text { [circumference area of bail bar] }+ \text { [circumference area of lug] } \\
\text { (note [end area of lug exposed] - [end area of lug against bail bar] } \\
\text { cancel one another and circumference area of bail bar covered by } \\
\text { lug is greater than the end area of lug exposed) }=\left[2 * \pi^{*} 0.5^{*} \mathrm{~h}\right]+ \\
{\left[0.5^{*} 0.563^{*} \cos ^{-1}(1-\mathrm{h} / 0.25)\right]}\end{array}$ \\
\hline
\end{tabular}


Table A-4. Outer Surface Area Calculation for DABT or L-Bundle

\begin{tabular}{|c|c|c|c|c|c|c|c|}
\hline \begin{tabular}{|c|} 
Scaling \\
Factor for \\
Height h \\
{$\left[\right.$ in $^{2} /$ in] $]$} \\
\end{tabular} & \begin{tabular}{|c|} 
Height h \\
[in]
\end{tabular} & $\begin{array}{c}\text { Constant } \\
{\left[\text { in }^{2}\right]}\end{array}$ & $\begin{array}{l}\text { Cumulative } \\
\text { Surface } \\
\text { Area }\left[i^{2}\right] \\
\end{array}$ & $\begin{array}{c}\text { From } \\
\text { [in] }\end{array}$ & To [in] & Description & Surface Area Sums \\
\hline 4.91 & 0.25 & 2226.20 & 2227.43 & 138.25 & 138.50 & $\begin{array}{l}\text { from middle of bail bar } \\
\text { lugs up to top of bail bar } \\
\text { lugs }\end{array}$ & $\begin{array}{c}\text { [circumference area of bail bar] }+ \text { [circumference area of lug] } \\
\text { (note [end area of lug exposed] - [end area of lug against bail bar] } \\
\text { cancel one another and circumference area of bail bar covered by } \\
\text { lug is greater than end area of lug exposed) }=\left[2 * \pi^{*} 0.5^{*} \mathrm{~h}\right]+ \\
{\left[0.563 *\left(\pi / 2-\cos ^{-1}(\mathrm{~h} / 0.25)\right) * 0.5\right]}\end{array}$ \\
\hline 3.14 & 1.00 & 2227.43 & 2230.57 & 138.50 & 139.50 & $\begin{array}{l}\text { from top of bail bar lugs } \\
\text { up to start of arc on bail } \\
\text { bar }\end{array}$ & {$[$ circumference area of bail bar $]=\left[2 * \pi^{*} 0.5^{*} \mathrm{~h}\right]$} \\
\hline 4.11 & 1.50 & 2230.57 & 2236.74 & 139.50 & 141.00 & $\begin{array}{l}\text { from start of arc on bail } \\
\text { bar up to top of bail bar }\end{array}$ & $\begin{array}{l}\text { [fraction of bail bar arc SA covered] (note treat circumference } \\
\text { area of arc as } 2 \text { straight pieces each with half length of } 180^{\circ} \text { arc } \\
\left.\text { with diameter of } 2.5^{\prime \prime}\right)=\left[\mathrm{h} / 1.5^{*} \pi / 2^{*} 2.5^{*} \pi^{*} 0.5\right]\end{array}$ \\
\hline
\end{tabular}




\section{A.2. $\quad$ Sodium Reactor Experiment (SRE) Surface Area Calculations}

The surface area per unit height of the Sodium Reactor Experiment (SRE) shipping can as described in the SRNS drawing EX-N704-000065 was derived. A sketch showing the side view of the SRE is shown in Figure A-2. The numbers along the right side of the drawing show the various layers of fuel slugs stacked end to end. A sketch showing the top cross-section of the SRE is in Figure A-3 where the red circles represent the $0.75 \mathrm{in}$. outer diameter fuel slugs, the green circles represent the $1 \mathrm{in}$. outer diameter aluminum spacer tubes, and the blue circles are the $1.125 \mathrm{in}$. outer diameter aluminum spacer tubes. A sketch of the lifting bail on top of the SRE shipping can is shown in Figure A-4. The various parts of the SRE have been color coded and the dimensions of these parts are shown in Table A-5.

Table A-5. Sodium Reactor Experiment (SRE) Shipping Can Parts and Dimensions

\begin{tabular}{|c|c|c|c|c|}
\hline L-Bundle Part & $\begin{array}{l}\text { Outer } \\
\text { Diameter } \\
\text { (OD) [in] }\end{array}$ & $\begin{array}{l}\text { Wall } \\
\text { [in] }\end{array}$ & $\begin{array}{c}\text { Length } \\
\text { (vertical } \\
\text { height) [in] }\end{array}$ & Comments \\
\hline Lifting Bail & 3.44 & $0.75^{*}$ & 5.750 & Detail dimension in Figure A-4 \\
\hline Lid Top & 5.080 & 0.052 & 0.250 & $\begin{array}{c}\text { Whole Lid overlaps flush with top of } \\
\text { Bundle Tube so count as part of Bundle } \\
\text { Tube Height. Indicate Lid Top Thickness } \\
\text { for potential inside surface area } \\
\text { calculations. }\end{array}$ \\
\hline External Bundle Tube & 3.500 & 0.125 & 110.250 & No holes or cuts in external bundle tube. \\
\hline Large Internal Aluminum Spacer Tubes & 1.125 & 0.028 & 103.000 & Two of these. \\
\hline Small Internal Aluminum Spacer Tubes & 1.000 & 0.028 & 103.000 & Five of these. \\
\hline Internal Fuel Slug & 0.750 & NA & 6.000 & $\begin{array}{l}\text { Stacked end to end in internal aluminum } \\
\text { spacer tubes. Maximum number per SRE } \\
\text { shipping can is } 119 \text {. }\end{array}$ \\
\hline Bottom Plate & 3.250 & NA & 0.500 & $\begin{array}{l}\text { Bottom plate recessed up into external } \\
\text { bundle tube except for } 0.12 " \text { exposed. }\end{array}$ \\
\hline
\end{tabular}

NA=Not Applicable, $*$ Leg Thickness

The SRE shipping can has no holes or cuts in the external bundle tube. For this reason the surface area calculations only consider the external surfaces. Starting from the bottom of the SRE and going up vertically the exposed outer surface area is calculated as shown in Table A-6. This table details the external surface area calculations for specific sections of the SRE in terms of height from its bottom. The same terms and structure as defined for the L-Bundle above are used in this table. Using the logic outlined in Table A-6, the total outer surface area for the SRE for the full 110.245 inch height was $1198.21 \mathrm{in}^{2}$ giving an overall outer scaling factor for the SRE of $10.87 \mathrm{in}^{2} / \mathrm{in}$ or $0.91 \mathrm{ft} / \mathrm{ft}$.

If one wanted to evaluate the additional area from the internal parts of the SRE assembly, the calculations in Table A- 6 would need to be augmented. After careful examination of the internal spacing between the aluminum spacer tubes and the fuel slugs, most of the internal clearances are less than $9.7 \mathrm{~mm}(\sim 3.18-$ $9.65 \mathrm{~mm}$ ) but there was a small portion of two of the one inch aluminum space tubes (No. 3 and 4 ) and a small portion of the main bundle tube with a clearance of about $12.2 \mathrm{~mm}$. Therefore the internal area that would possibly be exposed if there were cuts or holes in the SRE main bundling tube is not significant but is quantified in Table A-7. Note that the internal surface area terms that are not counted in the calculation are shown as strike through in Table A-7 for completeness. The additional inner surface area for the SRE for the full 110.245 inch height was $153.34 \mathrm{in}^{2}$ giving an overall inner scaling factor for the SRE of 1.47 
$\mathrm{in}^{2} /$ in or $0.12 \mathrm{ft}^{2} / \mathrm{ft}$. If the outer scaling factor of $0.91 \mathrm{ft}^{2} / \mathrm{ft}$ is combined with the inner scaling factor of $0.12 \mathrm{ft}^{2} / \mathrm{ft}$ the total inner and outer scaling factor becomes $1.03 \mathrm{ft}^{2} / \mathrm{ft}$.

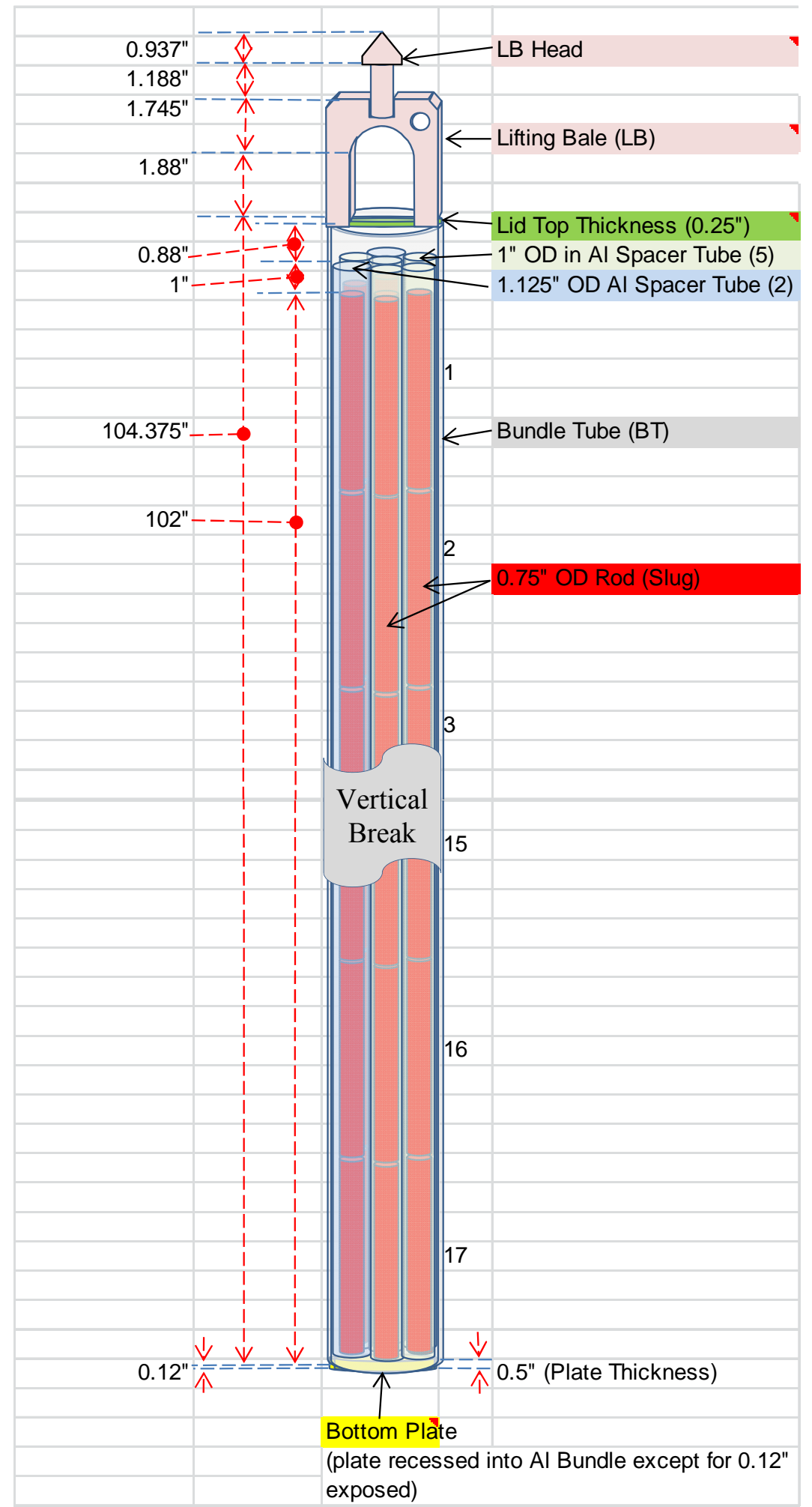

Figure A-2. Sketch of Sodium Reactor Experiment (SRE) Shipping Can 
SRNL-STI-2012-00279

Revision 1

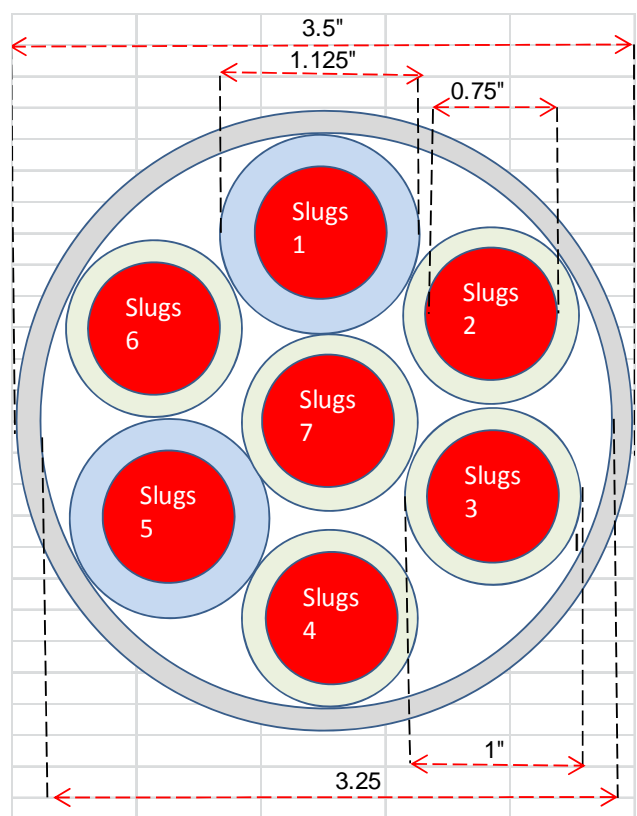

Figure A-3. Sketch of Cross-section of Sodium Reactor Experiment (SRE) Shipping Can

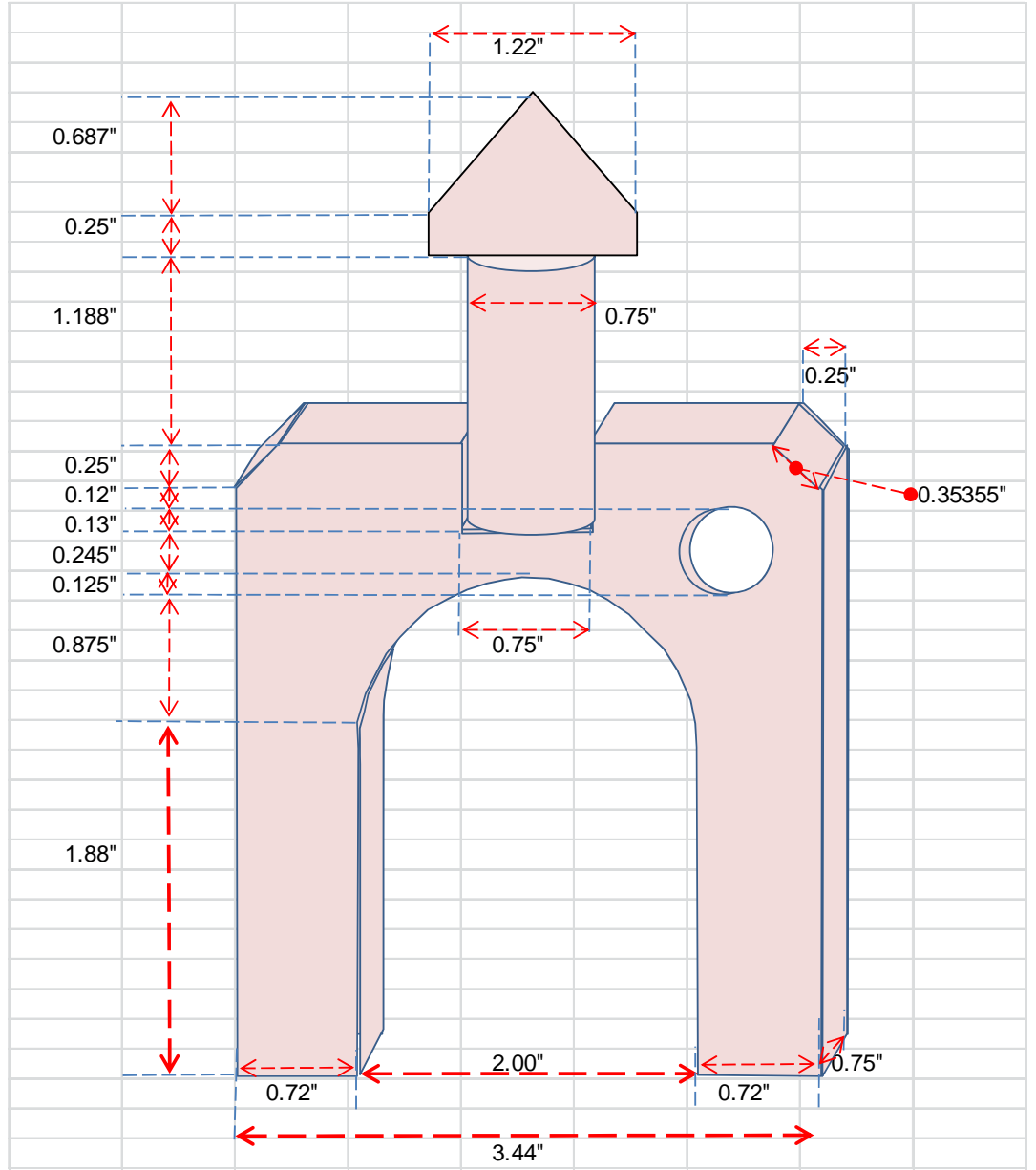

Figure A-4. Sketch of Lifting Bail of Sodium Reactor Experiment (SRE) Shipping Can 
Table A-6. Outer Surface Area Calculation for SRE

\begin{tabular}{|c|c|c|c|c|c|c|c|}
\hline \begin{tabular}{|c|} 
Scaling \\
Factor for \\
Height h \\
[in $/$ in] \\
\end{tabular} & \begin{tabular}{|c|} 
Height h \\
[in]
\end{tabular} & $\begin{array}{c}\text { Constant } \\
{\left[\text { in }^{2}\right]}\end{array}$ & \begin{tabular}{|l|} 
Cumulative \\
Surface \\
Area $\left[i^{2}\right]$ \\
\end{tabular} & $\begin{array}{c}\text { From } \\
\text { [in] }\end{array}$ & To [in] & Description & Surface Area Sums \\
\hline 10.21 & 0.12 & 8.30 & 9.52 & 0.00 & 0.12 & $\begin{array}{c}\text { From bottom of bottom plate up to start } \\
\text { of external Bundle Tube. }\end{array}$ & $\begin{array}{c}\text { [Circumference area of bottom plate }]+[\text { Surface } \\
\text { area of bottom plate }]=\left[\pi^{*} 3.25 * h\right]+\left[\pi^{*}(3.25 / 2)^{2}\right]\end{array}$ \\
\hline 11.00 & 104.38 & 9.52 & 1157.18 & 0.12 & 104.50 & $\begin{array}{c}\text { From start of external Bundle Tube up } \\
\text { to top of lid. Ignores small } 0.125^{\prime \prime} \text { high } \\
45^{\circ} \text { chamfer in lid and lid counted as } \\
\text { part of external Bundle Tube since sits } \\
\text { flush with outside wall of external } \\
\text { Bundle Tube. }\end{array}$ & $\begin{array}{l}\text { [Circumference area of external Bundle Tube and } \\
\qquad \text { Lid }]=\left[\pi^{*} 3.5^{*} \mathrm{~h}\right]\end{array}$ \\
\hline 5.88 & 1.88 & 1164.62 & 1175.68 & 104.50 & 106.38 & $\begin{array}{c}\text { From start of exposed Lifting Bale (LB) } \\
\text { legs in Al lid up to start of arc in LB. }\end{array}$ & $\begin{array}{c}{[(\text { front/back faces of LB leg }+ \text { right/left faces of LB }} \\
\left.\text { leg })^{*} \text { No. of LB Legs }\right]+[\text { surface area of top of lid }] \\
=\left[2 *\left(2 * 0.72 * \mathrm{~h}+2 * 0.75^{*} \mathrm{~h}\right)\right]+\left[\pi^{*}((3.5-0.75) / 2)^{2}+\right. \\
2 * 0.75]\end{array}$ \\
\hline 6.80 & 0.88 & 1175.68 & 1181.63 & 106.38 & 107.25 & $\begin{array}{c}\text { from start of arc in Lifting Bale (LB) up } \\
\text { to start of } 0.5 \text { " diameter hole in right } \\
\text { side of LB }\end{array}$ & $\begin{array}{c}\text { [inside SA of LB arc] }+ \text { [outside right/left faces of } \\
\text { LB legs] }+[\text { front/back faces of LB legs] }= \\
{\left[2 * 0.75^{*} \sin ^{-1}(\mathrm{~h})\right]+[2 * 0.75 * \mathrm{~h}]+[2 *\{\mathrm{~h} * 3.44-} \\
\left.\left.\sin ^{-1}(\mathrm{~h})-\mathrm{h} * \sqrt{1-\mathrm{h}^{2}}\right)\right]\end{array}$ \\
\hline 26.03 & 0.13 & 1181.63 & 1184.88 & 107.25 & 107.38 & $\begin{array}{c}\text { from start of } 0.5 " \text { diameter hole in right } \\
\text { side of LB up to top of arc in LB }\end{array}$ & $\begin{array}{c}-[\text { face area of hole] }+[\text { inside area of hole }]+ \\
\text { [outside right/left faces of LB legs] }+[\text { front/back } \\
\text { faces of LB legs] }+[\text { inside SA of LB arc }]=- \\
{\left[0.25^{2} * \cos ^{-1}(1-4 * \mathrm{~h})-(0.25-\mathrm{h}) * 0.25^{*} \sin \left(\cos ^{-1}(1-\right.\right.} \\
4 * \mathrm{~h}))]+\left[2 * 0.75^{*} \cos ^{-1}(1-4 * \mathrm{~h})\right]+\left[\mathrm{h} * 0.75^{*} 2\right]+ \\
{\left[2\left\{\mathrm{~h} * 3.44-\mathrm{h} / 0.125^{*} 0.04179\right\}\right]+} \\
{\left[\mathrm{h} / 0.125^{*} 0.50536 * 0.75 * 2\right]}\end{array}$ \\
\hline 14.18 & 0.13 & 1184.88 & 1186.66 & 107.38 & 107.50 & $\begin{array}{l}\text { from top of arc in LB up to middle of } \\
0.5^{\prime} \text { diameter hole in right side LB }\end{array}$ & $\begin{array}{c}\text { [front/back faces of LB legs] + [outside right/left } \\
\text { faces of LB legs] -[face area of hole in new section] } \\
+ \text { [inside area of hole in new section] }=[2 * \mathrm{~h} * 3.44] \\
+[2 * \mathrm{~h} * 0.75]-\left[\left(0.25^{2} * \cos ^{-1}(1-4 *(\mathrm{~h}+0.125))-\right.\right. \\
\mathrm{h} *(0.25-\mathrm{h}-0.125)^{*} 0.25^{*} \sin \left(\cos ^{-1}(1-4 *(\mathrm{~h}+\right. \\
0.125)))-\left(0.25^{2} * \cos ^{-1}(1-4 * 0.125)-(0.25-\right. \\
\left.\left.0.125) * 0.25^{*} \sin \left(\cos ^{-1}(1-4 * 0.125)\right)\right)\right]+ \\
{\left[2 * 0.75^{*} \cos ^{-1}(1-4 *(\mathrm{~h}+0.125))-2 * 0.75^{*} \cos ^{-1}(1-\right.} \\
4 * 0.125)]\end{array}$ \\
\hline
\end{tabular}


Table A-6. Outer Surface Area Calculation for SRE

\begin{tabular}{|c|c|c|c|c|c|c|c|}
\hline \begin{tabular}{|c|} 
Scaling \\
Factor for \\
Height h \\
{$\left[\right.$ in $^{2} /$ in] $]$} \\
\end{tabular} & \begin{tabular}{|l} 
Height h \\
[in]
\end{tabular} & $\begin{array}{c}\text { Constant } \\
{\left[\text { in }^{2}\right]}\end{array}$ & $\begin{array}{l}\text { Cumulative } \\
\text { Surface } \\
\text { Area }\left[i^{2}\right] \\
\end{array}$ & $\begin{array}{l}\text { From } \\
\text { [in] }\end{array}$ & To [in] & Description & Surface Area Sums \\
\hline 9.46 & 0.12 & 1186.66 & 1187.79 & 107.50 & 107.62 & $\begin{array}{l}\text { from middle of } 0.5 " \text { diameter hole in } \\
\text { right side LB up to bottom of notch in } \\
\text { LB }\end{array}$ & $\begin{array}{c}\text { [front/back faces of LB legs] }+ \text { [outside right/left } \\
\text { faces of LB legs] }-[\text { face area of hole in new section] } \\
+\left[\text { inside area of hole in new section] }=\left[2 * h^{*} 3.44\right]\right. \\
\left.+\left[2 * h^{*} 0.75\right]-\left[0.25^{2} * \sin ^{-1}(4 * \mathrm{~h})+\mathrm{h} * \sqrt{0.25^{2}-\mathrm{h}^{2}}\right)\right] \\
+\left[2 * 0.75^{*} 0.25^{*} \sin -1(4 * \mathrm{~h})\right]\end{array}$ \\
\hline 11.00 & 0.13 & 1187.79 & 1189.22 & 107.62 & 107.75 & $\begin{array}{c}\text { from bottom of notch in LB up to top of } \\
0.5^{\prime \prime} \text { diameter hole on right side LB }\end{array}$ & $\begin{array}{c}\text { [front/back faces LB legs] }+ \text { [outside right/left faces } \\
\text { LB legs]-[face area of hole in new section] }+[\text { inside } \\
\text { area of hole in new section] }+ \text { [notch area bottom } \\
\text { exposed] }+[\text { head circumference area }]+[\text { notch side } \\
\text { area exposed }]=[3.44 * \mathrm{~h}-0.75 * \mathrm{~h}]+[0.75 * \mathrm{~h}]- \\
{[\mathrm{h} / 0.13 * 0.04057]+[\mathrm{h} / 0.13 * 0.4013]+\left[0.75^{2} *(1-\pi\right.} \\
/ 4)]+[0.75 * \pi * \mathrm{~h}]+[2 * \mathrm{~h} * 0.75]\end{array}$ \\
\hline 7.30 & 0.12 & 1189.22 & 1190.10 & 107.75 & 107.87 & $\begin{array}{l}\text { from top of } 0.5^{\prime \prime} \text { diameter hole on right } \\
\text { side LB up to } 45^{\circ} \text { chamfer start on LB }\end{array}$ & $\begin{array}{c}{[\text { front/back faces LB legs }]+[\text { outside right/left faces }} \\
\text { LB legs }]+[\text { head circumference area }]+[\text { notch side } \\
\text { area exposed }]=[3.44 * \mathrm{~h}-0.75 * \mathrm{~h}]+[0.75 * \mathrm{~h}]+ \\
{[0.75 * \pi * \mathrm{~h}]+[2 * \mathrm{~h} * 0.75]}\end{array}$ \\
\hline 7.48 & 0.25 & 1190.10 & 1191.97 & 107.87 & 108.12 & $\begin{array}{l}\text { from } 45^{\circ} \text { chamfer start on LB up to top } \\
\text { of LB }\end{array}$ & $\begin{array}{c}\text { [front/back faces LB legs] }+[\text { head circumference } \\
\text { area }]+[\text { notch side area exposed }]-[\text { chamfer missing } \\
\text { face area }]+[\text { chamfer side area }]=[3.44 * \mathrm{~h}-0.75 * \mathrm{~h}] \\
+[0.75 * \pi * \mathrm{~h}]+[2 * \mathrm{~h} * 0.75]-\left[0.5 * \mathrm{~h}^{2}\right]+ \\
{[0.75 * \mathrm{~h} * \sqrt{2}]}\end{array}$ \\
\hline 2.36 & 1.19 & 1191.97 & 1194.77 & 108.12 & 109.31 & $\begin{array}{l}\text { from top of LB up to start of conical } \\
\text { section of head }\end{array}$ & {$[$ head circumference area $]=\left[0.75^{*} \pi^{*} \mathrm{~h}\right]$} \\
\hline 6.74 & 0.25 & 1194.77 & 1196.45 & 109.31 & 109.56 & $\begin{array}{c}\text { from start of conical section of head up } \\
\text { to top of flat sided portion of conical } \\
\text { section }\end{array}$ & $\begin{array}{c}\text { [area on underside of conical section] }+ \text { [flat section } \\
\text { of conical section side area] }=\left[\pi^{*}(1.22 / 2)^{2}-\right. \\
\left.\pi^{*}(0.75 / 2)^{2}\right]+[1.22 * \pi * h]\end{array}$ \\
\hline 2.56 & 0.69 & 1196.45 & 1198.21 & 109.56 & 110.25 & $\begin{array}{c}\text { from top of flat sided portion of conical } \\
\text { section up to conical apex }\end{array}$ & $\begin{array}{c}{[\text { conical surface area }]=\left[\pi^{*} 0.61 * 0.91873-\right.} \\
\left.\pi^{*} 0.66396^{*}(0.687-\mathrm{h})^{2} / 0.74777^{2}\right]\end{array}$ \\
\hline
\end{tabular}


Table A-7. Internal Surface Area Calculation for SRE

\begin{tabular}{|c|c|c|c|c|c|c|c|}
\hline \begin{tabular}{|c|} 
Scaling \\
Factor for \\
Height h \\
[in $^{2} /$ in] \\
\end{tabular} & \begin{tabular}{|c|} 
Height h \\
[in]
\end{tabular} & $\begin{array}{c}\text { Constant } \\
{\left[\text { in }^{2}\right]}\end{array}$ & $\begin{array}{c}\text { Cumulative } \\
\text { Surface } \\
\text { Area }\left[i^{2}\right]\end{array}$ & $\begin{array}{c}\text { From } \\
\text { [in] }\end{array}$ & To [in] & Description & Surface Area Sums \\
\hline 0.00 & 0.50 & & 0.00 & 0.00 & 0.50 & $\begin{array}{l}\text { from outside bottom plate up to top of } \\
\text { bottom plate }\end{array}$ & Already accounted for in external calculation. \\
\hline 1.35 & 102.00 & 3.09 & 140.66 & 0.50 & 102.50 & $\begin{array}{l}\text { from top of bottom plate up to top of } \\
\text { fuel slugs inside Al tubes }\end{array}$ & $\begin{array}{c}\text { [circumference area of inside main Al canister] }+ \\
\text { [circumference area of outside Al tubes 1-7] }+ \\
\text { [circumference area of inside Al tubes } 1-7]+ \\
\text { [circumference area of outside fuel slugs } 1-7]+ \\
\text { [bottom surface area of fuel slugs } 1-7] \text { assumes fuel } \\
\text { slugs flush top to bottom and contact points between } \\
\text { inside Al tubes and Al canister and fuel slugs } \\
\text { negligible and anything closer than } 9.7 \mathrm{~mm} \text { ignore }= \\
{[0.29647 * 1.625 * \mathrm{~h}]\{\text { only portion }>9.7 \mathrm{~mm}\}+} \\
{\left[2 *(0.86689 * 0.5)^{*} \mathrm{~h}\right]\{\text { only portion }>9.7 \mathrm{~mm}\}+} \\
{\left[7 * \pi *(0.75 / 2)^{2}\right]}\end{array}$ \\
\hline 1.35 & 1.00 & 143.75 & 145.10 & 102.50 & 103.50 & $\begin{array}{l}\text { from top of fuel slugs inside Al tubes to } \\
\text { top of Al tubes }\end{array}$ & $\begin{array}{c}\text { [circumference area of inside main Al canister] }+ \\
\text { [circumference area of outside Al tubes 1-7] }+ \\
\text { [circumference area of inside Al tubes } 1-7]+[\text { top } \\
\text { surface area of fuel slugs } 1-7] \text { assumes fuel slugs } \\
\text { flush top to bottom and contact points between } \\
\text { inside Al tubes and Al canister and fuel slugs } \\
\text { negligible }=[0.29647 * 1.625 * \mathrm{~h}] \text { \{only portion }>9.7 \\
\mathrm{~mm}\}+[2 *(0.86689 * 0.5) * \mathrm{~h}] \text { \{only portion }>9.7 \\
\mathrm{~mm}\}+\left[7 * \pi *(0.75 / 2)^{2}\right]\end{array}$ \\
\hline 9.42 & 0.88 & 145.10 & 153.34 & 103.50 & 104.38 & from top of $\mathrm{Al}$ tubes to top inside of lid & [circumference area of inside $\mathrm{Al} \mathrm{lid}]=\left[3.00^{*} \pi^{*} \mathrm{~h}\right]$ \\
\hline
\end{tabular}




\section{A.3. $\quad$ Hahn-Meitner-Institut (HMI) Surface Area Calculations}

There were two types of Hahn-Meitner-Institut (HMI) assemblies, a standard and control assembly. The surface area calculations for the standard HMI assembly will be described first followed by the control assembly.

\section{A.3.1. Standard HMI Assembly Surface Area Calculations}

The standard HMI assembly is described in the SRNS drawings 2598-100, 101, 102, 104, 105, 300, 303, 304, and 305. The standard HMI assemblies described in these drawings were then cropped by SRNS as described in the calculation sheet M-CLC-L- $00285^{32}$. A sketch showing the side view of the standard HMI assembly is shown in Figure A-5. An enlarged sketch of the standard HMI assembly with the front side plate removed is shown in Figure A-6. The various parts of the standard HMI assembly have been color coded and the dimensions of these parts are shown in Table A-8.

Table A-8. Standard HMI Assembly Parts and Dimensions

\begin{tabular}{|c|c|c|c|c|}
\hline & & & & \\
HMI Part & Height [in] & Length (width) [in] & Depth[in] & Comments \\
\hline Side Plate & 3.37 & 28.48 & 0.18 & Front and Back Side Plates \\
\hline External Fuel Plate & 0.06 & 27.30 & 2.79 & 2 External Plates \\
\hline External Fuel Plate Core & 0.06 & 24.02 & 2.79 & 2 External Plates \\
\hline Internal Fuel Plate & 0.05 & 24.61 & 2.79 & 21 Internal Plates \\
\hline Internal Fuel Plate Core & 0.05 & 24.02 & 2.79 & 21 Internal Plates \\
\hline
\end{tabular}

Since the original standard HMI assembly was cropped, some internal surfaces will be exposed when placed in the L-Bundle. Starting from the bottom of the standard HMI assembly and going up vertically the exposed surface area is calculated as shown in Table A-9. This table as the earlier ones details the surface area calculations for specific sections of the standard HMI assembly in terms of height from its bottom. The same terms and structure as defined for the L-Bundle and SRE above are used in this table. Note that the clearances between the internal and external fuel plates are about $2.1 \mathrm{~mm}$ and so these internal surfaces should not be counted in the surface area calculations. Using the logic outlined in Table A-9, the total surface area for the standard HMI assembly for the full 26 inch height was $345.71 \mathrm{in}^{2}$ giving an overall outer scaling factor for the standard HMI assembly of $13.30 \mathrm{in}^{2} / \mathrm{in}$ or $1.11 \mathrm{ft}^{2} / \mathrm{ft}$. 


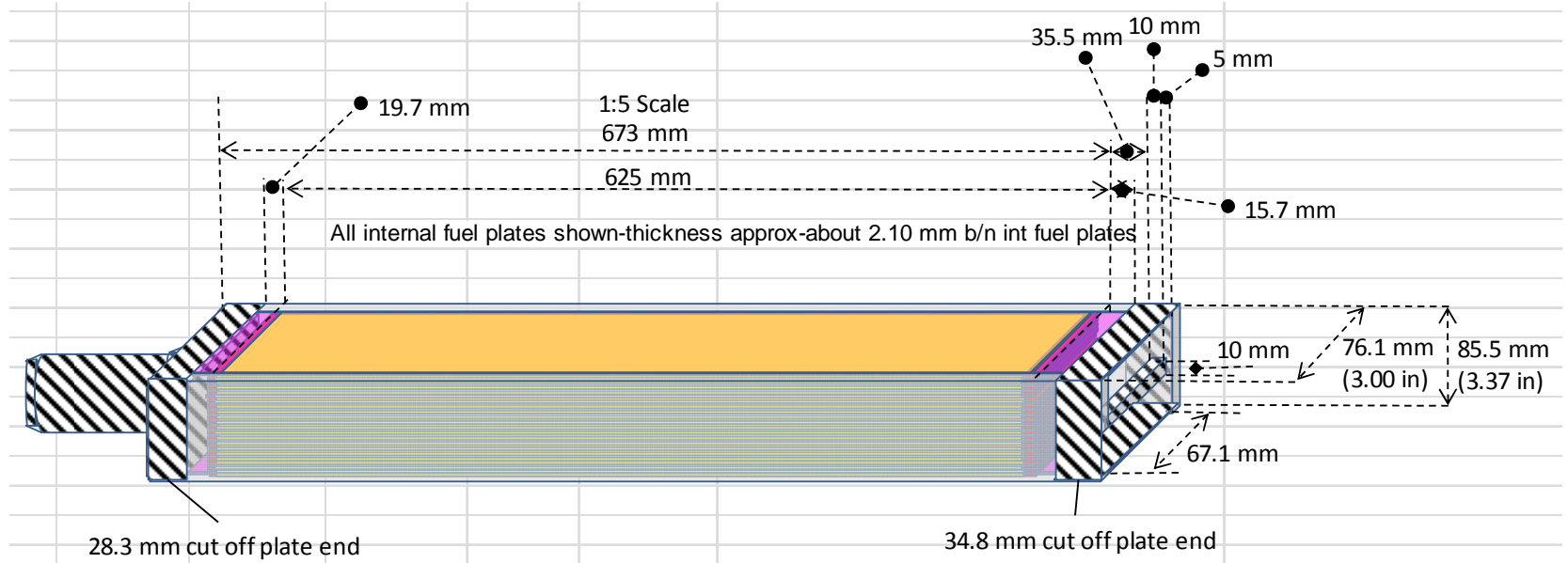

Figure A-5. Sketch of Standard HMI Assembly with Cropped Sections Cross Hatched

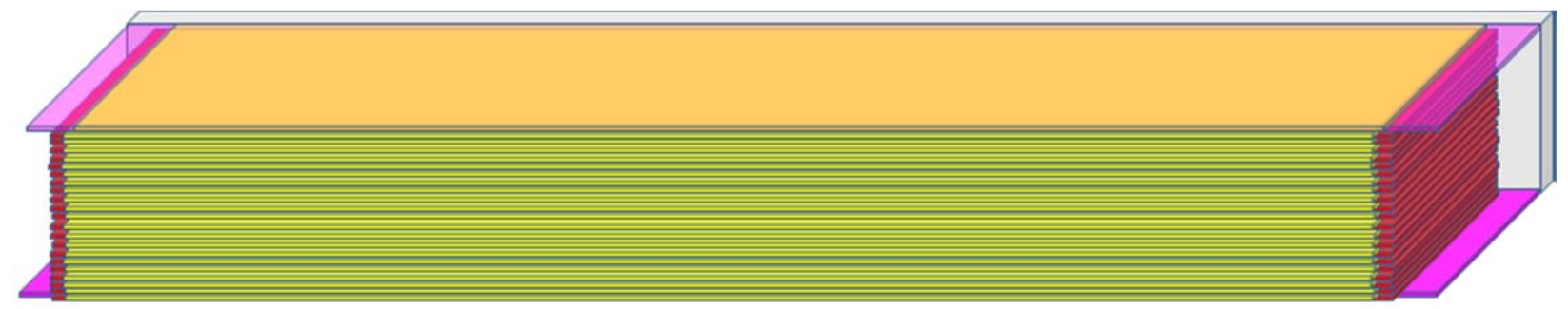

Figure A-6. Sketch of Standard HMI Assembly with Side Plate Removed 
Table A-9. Surface Area Calculation for Standard HMI Assembly

\begin{tabular}{|c|c|c|c|c|c|c|c|}
\hline \begin{tabular}{|c|} 
Scaling \\
Factor for \\
Height h \\
{$\left[\right.$ in $^{2} /$ in] } \\
\end{tabular} & $\begin{array}{l}\text { Height h } \\
\text { [in] }\end{array}$ & $\begin{array}{c}\text { Constant } \\
{\left[\text { in }^{2}\right]}\end{array}$ & $\begin{array}{c}\text { Cumulative } \\
\text { Surface Area } \\
{\left[\text { in }^{2}\right]}\end{array}$ & $\begin{array}{c}\text { From } \\
\text { [in] }\end{array}$ & To [in] & Description & Surface Area Sums \\
\hline 25.45 & 0.62 & 1.50 & 17.23 & 0.00 & 0.62 & $\begin{array}{l}\text { from start of cropped end up to start of } \\
\text { internal fuel plates }\end{array}$ & $\begin{array}{c}\text { [bottom edges of side plates] }+ \text { [bottom edges of } \\
\text { outer fuel plates] }+ \text { [outside side plates] }+ \text { [inside } \\
\text { side plates] }+[\text { outside outer fuel plates] }+ \text { [inside } \\
\text { outer fuel plates] }]=[2 * 0.177 * 3.366]+ \\
{[2 * 0.059 * 2.642]+[3.366 * \mathrm{~h} * 2]+[3.366 * \mathrm{~h} * 2]+} \\
{[2.996 * \mathrm{~h} * 2]+[2.996 * \mathrm{~h} * 2]}\end{array}$ \\
\hline 12.72 & 24.61 & 19.99 & 333.09 & 0.62 & 25.22 & $\begin{array}{c}\text { from start of internal fuel plates up to top } \\
\text { of internal fuel plates (note ignore inside } \\
\text { area between internal fuel plates and outer } \\
\text { fuel plates since they are about } 2.1 \mathrm{~mm} \\
\text { apart) }\end{array}$ & $\begin{array}{c}\text { [bottom edge area of internal plates] }+[\text { outside } \\
\text { side plates] }+[\text { outside outer fuel plates] }= \\
{[21 * 0.05 * 2.62]+[3.366 * \mathrm{~h} * 2]+[2.996 * \mathrm{~h} * 2]}\end{array}$ \\
\hline 12.72 & 0.78 & 335.84 & 345.71 & 25.22 & 26.00 & $\begin{array}{l}\text { from top of internal fuel plates up to } \\
\text { cropped end }\end{array}$ & $\begin{array}{c}\text { [top edge area of internal plates] }+ \text { [outside side } \\
\text { plates] }+[\text { outside outer fuel plates] }= \\
{[21 * 0.05 * 2.62]+[3.366 * h * 2]+[2.996 * h * 2]}\end{array}$ \\
\hline
\end{tabular}




\section{A.3.2. $\quad$ Control HMI Assembly Surface Area Calculations}

The control HMI assembly is described in the SRNS drawings 2598-400, 401, 402, 403, 405, 201, 202 , 203, 205, and 207. The control HMI assemblies described in these drawings were then cropped by SRNS as described in the calculation sheet M-CLC-L- $00285^{32}$. A sketch showing the side view of the control HMI assembly is shown in Figure A-7. An enlargement of the cylindrical cropped end of the control HMI assembly is shown in Figure A-8. An enlarged sketch of the standard HMI assembly with the front side plate removed is shown in Figure A-9. The various parts of the standard HMI assembly have been color coded and the dimensions of these parts are shown in Table A-10.

Table A-10. Control HMI Assembly Parts and Dimensions

\begin{tabular}{|c|c|c|c|c|}
\hline HMI Part & $\begin{array}{c}\text { Height } \\
\text { [in] }\end{array}$ & $\begin{array}{c}\text { Length (width) } \\
\text { [in] }\end{array}$ & Depth[in] & Comments \\
\hline Side Plate & 3.37 & 31.79 & 0.18 & Front and Back Side Plates \\
\hline Outer Absorber Plate & 0.06 & 31.75 & 2.79 & 2 Outer Plates on Top and Bottom \\
\hline Inner Absorber Plate & 0.05 & 29.90 & 2.79 & 2 Inner Plates on Top and Bottom \\
\hline Internal Fuel Plate & 0.05 & 24.61 & 2.79 & 17 Internal Plates \\
\hline Internal Fuel Plate Core & 0.05 & 24.02 & 2.79 & 17 Internal Plates \\
\hline Cylindrical End -1 & 2.969 (OD) & 0.197 & NA & Cylindrical end closest to fuel plates (see \\
Figure A-8)
\end{tabular}

Since the original control HMI assembly was cropped, some internal surfaces will be exposed when placed in the L-Bundle. Starting from the bottom of the control HMI assembly and going up vertically the exposed surface area is calculated as shown in Table A-11. This table as the earlier ones details the surface area calculations for specific sections of the control HMI assembly in terms of height from its bottom. The same terms and structure as defined for the L-Bundle, SRE, and HMI-standard above are used in this table. Note that the clearances between the internal fuel plates and outer/inner absorber plates are about $2.1 \mathrm{~mm}$ and the clearance between the outer/inner absorber plates is about $6 \mathrm{~mm}$ so these internal surfaces should not be counted in the surface area calculations. Using the logic outlined in Table A-11, the total surface area for the control HMI assembly for the full 30.3 inch height was 399.72 $\mathrm{in}^{2}$ giving an overall outer scaling factor for the control HMI assembly of $13.19 \mathrm{in}^{2} /$ in or $1.10 \mathrm{ft}^{2} / \mathrm{ft}^{\text {. }}$ 


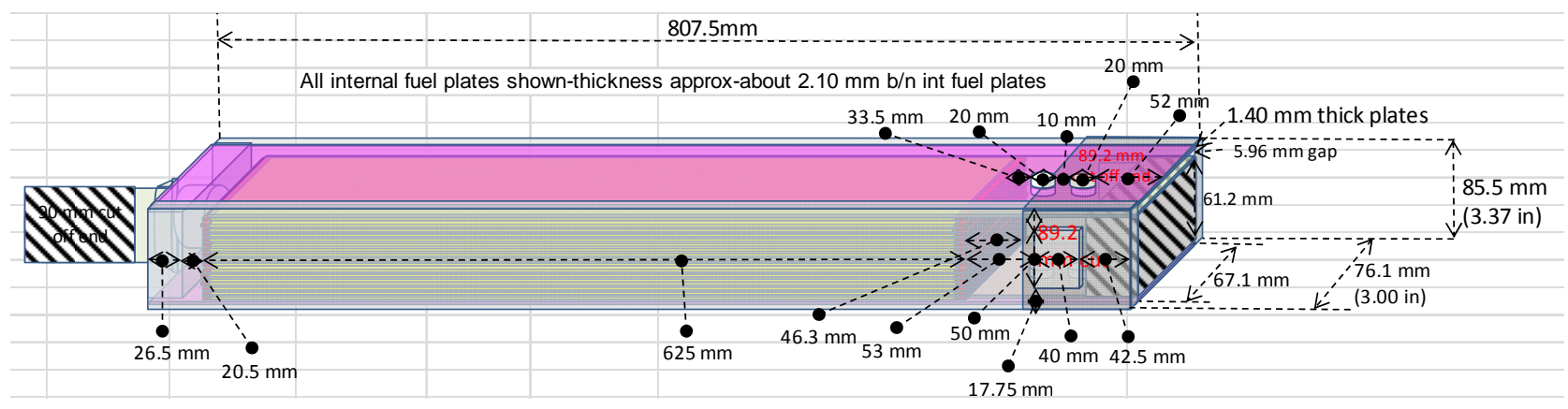

Figure A-7. Sketch of Control HMI Assembly with Cropped Sections Cross Hatched

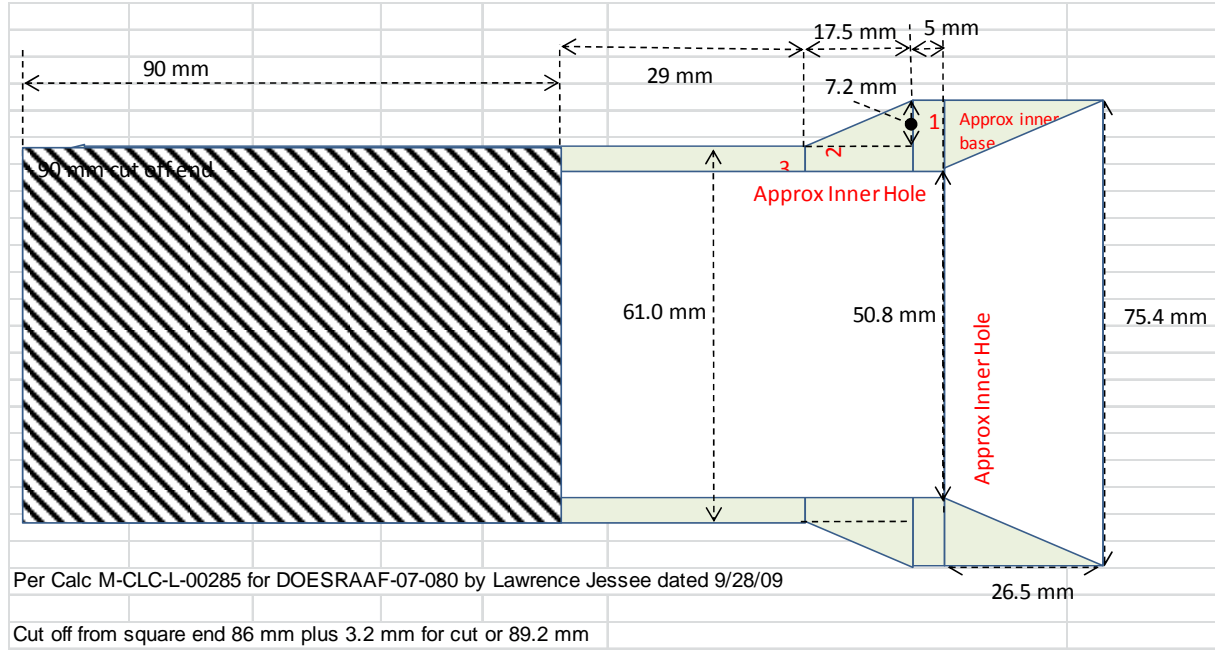

Figure A-8. Sketch of End of Control HMI Assembly with Cropped Sections Cross Hatched

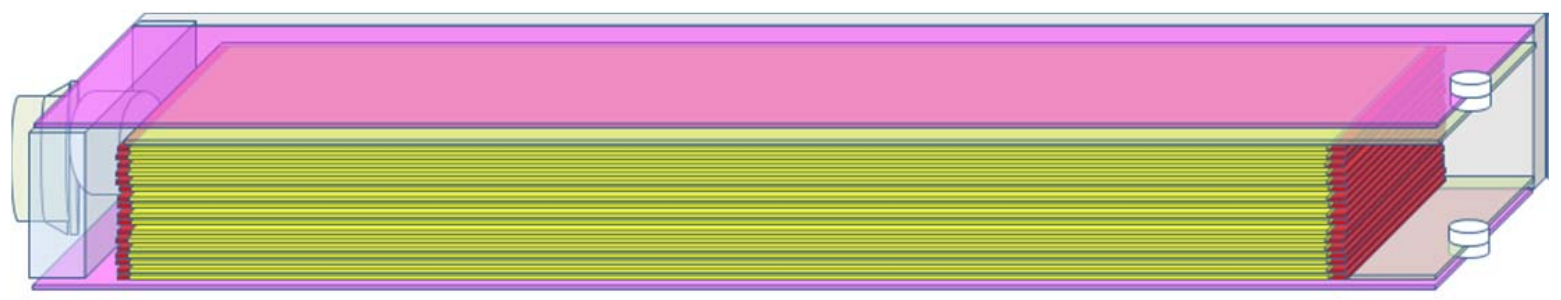

Figure A-9. Sketch of Control HMI Assembly with Front Side Plate Removed 
Table A-11. Surface Area Calculation for Control HMI Assembly

\begin{tabular}{|c|c|c|c|c|c|c|c|}
\hline $\begin{array}{l}\text { Scaling } \\
\text { Factor for } \\
\text { Height h } \\
{\left[\text { in }^{2} / \text { in] }\right.}\end{array}$ & $\begin{array}{l}\text { Height h } \\
\text { [in] }\end{array}$ & $\begin{array}{c}\text { Constant } \\
{\left[\mathrm{in}^{2}\right]}\end{array}$ & $\begin{array}{c}\text { Cumulative } \\
\text { Surface Area } \\
{\left[i^{2}\right]}\end{array}$ & $\begin{array}{c}\text { From } \\
\text { [in] }\end{array}$ & To [in] & Description & Surface Area Sums \\
\hline 22.53 & 0.11 & 1.78 & 4.26 & 0.00 & 0.11 & $\begin{array}{l}\text { from start of cropped end up } \\
\text { to middle of hole }\end{array}$ & $\begin{array}{c}\text { [bottom edges of outer and inner top \& bottom Abs plates] }+ \\
\text { [bottom edges of front \& back side plates] + [outside faces of } \\
\text { front \& back side plates] + [outside faces of top \& bottom outer } \\
\text { Abs plates] }+ \text { [outer faces of top \& bottom inner Abs plates] }+ \\
\text { [inner faces of side plates] - [face area of holes in outer top/bot } \\
\text { Abs plates] }+ \text { [inside circumference area of holes in out/in } \\
\text { top/bot Abs plates] }=[0.0551 * 2.642 * 2 * 2]+[0.177 * 3.366 * 2]+ \\
{\left[3.366^{*} \mathrm{~h}^{*} 2\right]+[2.996 * \mathrm{~h} * 2]+[2.642 * \mathrm{~h} * 2]+[2.409 * \mathrm{~h} * 2]-} \\
{\left[4 *\left\{0.394^{2} * \cos ^{-1}(1-\mathrm{h} / 0.394)-0.394 *(0.394-\mathrm{h}) * \sin \left(\cos ^{-1}(1-\right.\right.\right.} \\
\left.\mathrm{h} / 0.394))\}]+\left[4 * 2 * 0.394 * 0.0551^{*} \cos ^{-1}(1-\mathrm{h} / 0.394)\right)\right] \\
\end{array}$ \\
\hline 21.05 & 0.39 & 4.26 & 12.54 & 0.11 & 0.50 & $\begin{array}{l}\text { from middle of second hole } \\
\text { in out/in top/bot Abs plate } \\
\text { up to top of second hole }\end{array}$ & $\begin{array}{c}\text { [outside faces of front \& back side plates] }+ \text { [outside faces of } \\
\text { top \& bottom outer Abs plates] }+ \text { [outer faces of top \& bottom } \\
\text { inner Abs plates] }+ \text { [inner faces of side plates] - [face area of } \\
\text { holes in outer top/bot Abs plates] }+ \text { [inside circumference area } \\
\text { of holes in out/in top/bot Abs plates] }=\left[3.366^{*} \mathrm{~h} * 2\right]+ \\
{\left[2.996^{*} \mathrm{~h} 2\right]+[2.642 * \mathrm{~h} * 2]+[2.409 * \mathrm{~h} * 2]-} \\
{\left[4 *\left\{\mathrm{~h} * \sqrt{0.394^{2}-h^{2}}+0.394^{2} * \sin ^{-1}(\mathrm{~h} / 0.394)\right\}\right]+} \\
{\left[4 * 2 * 0.394 * 0.0551^{*} \sin ^{-1}(\mathrm{~h} / 0.394)\right]}\end{array}$ \\
\hline 22.83 & 1.32 & 12.54 & 42.65 & 0.50 & 1.82 & $\begin{array}{l}\text { from top of second hole in } \\
\text { out/in top/bot Abs plate up } \\
\text { to start of internal fuel plates }\end{array}$ & $\begin{array}{c}\text { [outside faces of front } \& \text { back side plates] }+[\text { outside faces of } \\
\text { top \& bottom outer Abs plates] }+[\text { outer faces of top \& bottom } \\
\text { inner Abs plates] }+[\text { inner faces of side plates] }=[3.366 * \mathrm{~h} * 2]+ \\
{[2.996 * \mathrm{~h} * 2]+[2.642 * \mathrm{~h} * 2]+[2.409 * \mathrm{~h} * 2]}\end{array}$ \\
\hline 12.72 & 24.61 & 44.88 & 357.98 & 1.82 & 26.43 & $\begin{array}{c}\text { from start of internal fuel } \\
\text { plates up to top of int fuel } \\
\text { plates (note don't count inner } \\
\text { faces due to closeness of } \\
\text { plates) }\end{array}$ & $\begin{array}{c}\text { [outside faces of front } \& \text { back side plates] }+ \text { [outside faces of } \\
\text { top \& bottom outer Abs plates] }+[\text { bottom edges of internal fuel } \\
\text { plates }]=\left[3.366^{*} h^{*} 2\right]+\left[2.996^{*} h^{*} 2\right]+\left[17^{*} 0.050^{*} 2.62\right]\end{array}$ \\
\hline 12.72 & 0.81 & 357.98 & 368.25 & 26.43 & 27.24 & $\begin{array}{c}\text { from top of int fuel plates up } \\
\text { to bottom of end fitting base } \\
\text { (don't include any internal } \\
\text { surfaces) }\end{array}$ & $\begin{array}{l}\text { [outside faces of front } \& \text { back side plates] }+[\text { outside faces of } \\
\text { top \& bottom outer Abs plates] }=\left[3.366^{*} h^{*} 2\right]+\left[2.996^{*} h^{*} 2\right]\end{array}$ \\
\hline
\end{tabular}


Table A-11. Surface Area Calculation for Control HMI Assembly

\begin{tabular}{|c|c|c|c|c|c|c|c|}
\hline \begin{tabular}{|c|} 
Scaling \\
Factor for \\
Height h \\
{$\left[\right.$ in $^{2} /$ in] }
\end{tabular} & $\begin{array}{l}\text { Height h } \\
\text { [in] }\end{array}$ & $\begin{array}{c}\text { Constant } \\
{\left[\mathrm{in}^{2}\right]}\end{array}$ & $\begin{array}{c}\text { Cumulative } \\
\text { Surface Area } \\
{\left[\text { in }^{2}\right]}\end{array}$ & $\begin{array}{c}\text { From } \\
\text { [in] }\end{array}$ & To [in] & Description & Surface Area Sums \\
\hline 12.72 & 1.04 & 368.25 & 381.52 & 27.24 & 28.28 & $\begin{array}{c}\text { from bottom of end fitting } \\
\text { base up to top of side/outer } \\
\text { Abs plates (don't include any } \\
\text { internal surfaces) }\end{array}$ & $\begin{array}{l}\text { [outside faces of front } \& \text { back side plates] }+[\text { outside faces of } \\
\text { top \& bottom outer Abs plates] }=\left[3.366^{*} h * 2\right]+\left[2.996^{*} h^{*} 2\right]\end{array}$ \\
\hline 9.33 & 0.20 & 383.01 & 384.84 & 28.28 & 28.48 & $\begin{array}{l}\text { from top of side/outer } \mathrm{Abs} \\
\text { plates up to top of part } 1 \text { of } \\
\text { end fitting cylinder }\end{array}$ & $\begin{array}{c}\text { [top area if no cylinder or corner holes] - }[\text { cross sectional area } \\
\text { of cylinder base] - }[\text { area of corner holes] }+[\text { circumference } \\
\text { outside area of end fitting cylinder }]=[2.969 * 2.929]- \\
{\left[\pi^{*}(2.969 / 2)^{2}\right]-[0.5 * 0.382 * 0.382 * 4]+\left[\pi^{*} 2.969 * \mathrm{~h}\right]}\end{array}$ \\
\hline 9.09 & 0.69 & 384.84 & 391.10 & 28.48 & 29.17 & $\begin{array}{c}\text { from top of part } 1 \text { of end } \\
\text { fitting cylinder to top of part } \\
2 \text { of end fitting (for } \\
\text { derivation see calc for Std } \\
\text { HMI) }\end{array}$ & $\begin{array}{c}{[\text { circumference area of conical section of part } 2]=[\text { total conical }} \\
\text { area }]-[\text { top conical area }]=\left[\left(\pi^{*} 1.484 * 4.34\right)-\left(\pi^{*} 0.342 /(1-\right.\right. \\
\left.\left.\left.0.342^{2}\right) *(4.078-\mathrm{h})^{2}\right)\right]\end{array}$ \\
\hline 7.54 & 1.14 & 391.10 & 399.72 & 29.17 & 30.31 & $\begin{array}{l}\text { from top of part } 2 \text { of end } \\
\text { fitting cylinder up to top of } \\
\text { part } 3 \text { of end fitting (note } \\
\text { calc ends here since cut off } \\
\text { top } 50 \mathrm{~mm} \text { ) }\end{array}$ & $\begin{array}{l}\text { [circumference area of cylindrical section of part } 3]= \\
\qquad\left[\pi^{*} 2.402 * h\right]\end{array}$ \\
\hline
\end{tabular}


SRNL-STI-2012-00279

Revision 1

\section{A.4. DR-3 (Denmark Reactor) Surface Area Calculations}

The surface area per unit height of the DR-3 (Denmark Reactor) assemblies as described in the SRNS drawings VPF 22923-001,002,003,004,005,006,007,008,009,010,011-A-RISO was derived. The original DR-3 assemblies were altered as shown in VPF 22923-001,002,003,004-A-RISO where only the concentric fuel tubes remained in a cup holder assembly. The fuel tubes were of two lengths where the first set is designated as DR-3-1 which was 24.606 inches long and a second set designated as DR-3-2 which was 26.0 inches long. Therefore, the surface area calculations have been broken down into two sections.

\section{A.4.1. $\quad$ DR-3-1 (Denmark Reactor) Surface Area Calculations}

A sketch of the side view of the DR-3-1 assembly is shown in Figure A-10. A top cross sectional view of the DR-3-1 assembly is shown in Figure A-11 showing the spacing of the concentric fuel tubes. Figure A-12 shows a cross-section side view of the DR-3-1 assembly with the top and bottom cup plates holding the fuel tubes with a hairpin. Figure A-13 shows a top view of the top and bottom cup plates. Since the cup plates are only $1 \mathrm{~mm}$ thick the inside area of holes are not counted in the surface area calculations. The elevation change in the top and bottom cup plates due to a $40.5 \mathrm{~mm}$ circular countersunk area around the center point is also ignored in the surface area calculations. The various parts of the DR-3-1 assembly have been color coded and the dimensions of these parts are shown in Table A-12. 
SRNL-STI-2012-00279

Revision 1

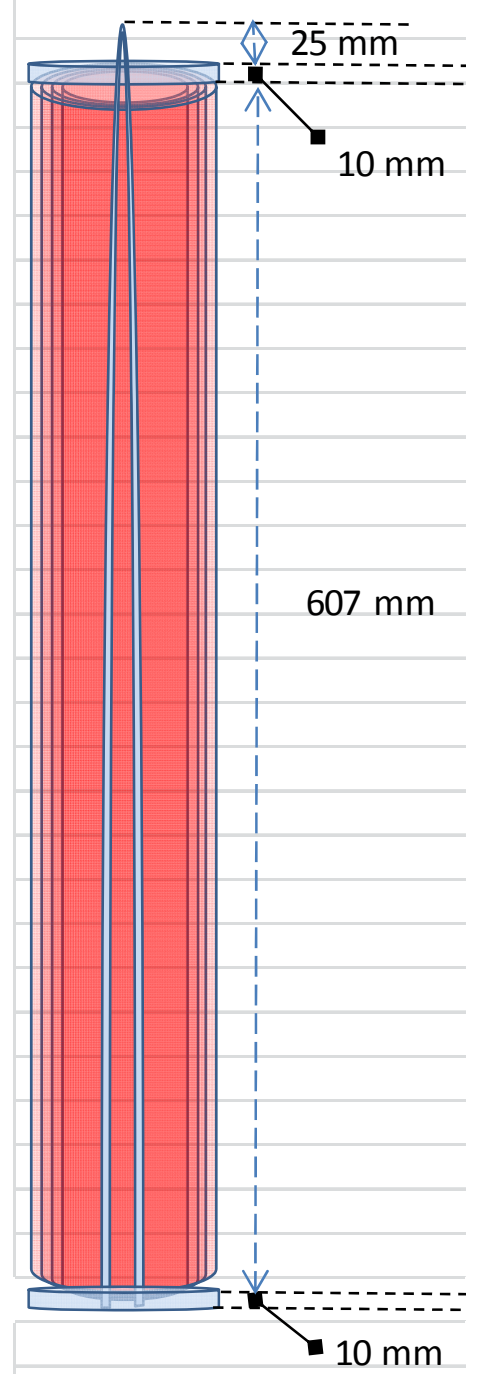

Figure A-10. Sketch of Side View of DR-3-1 Assembly 
SRNL-STI-2012-00279

Revision 1

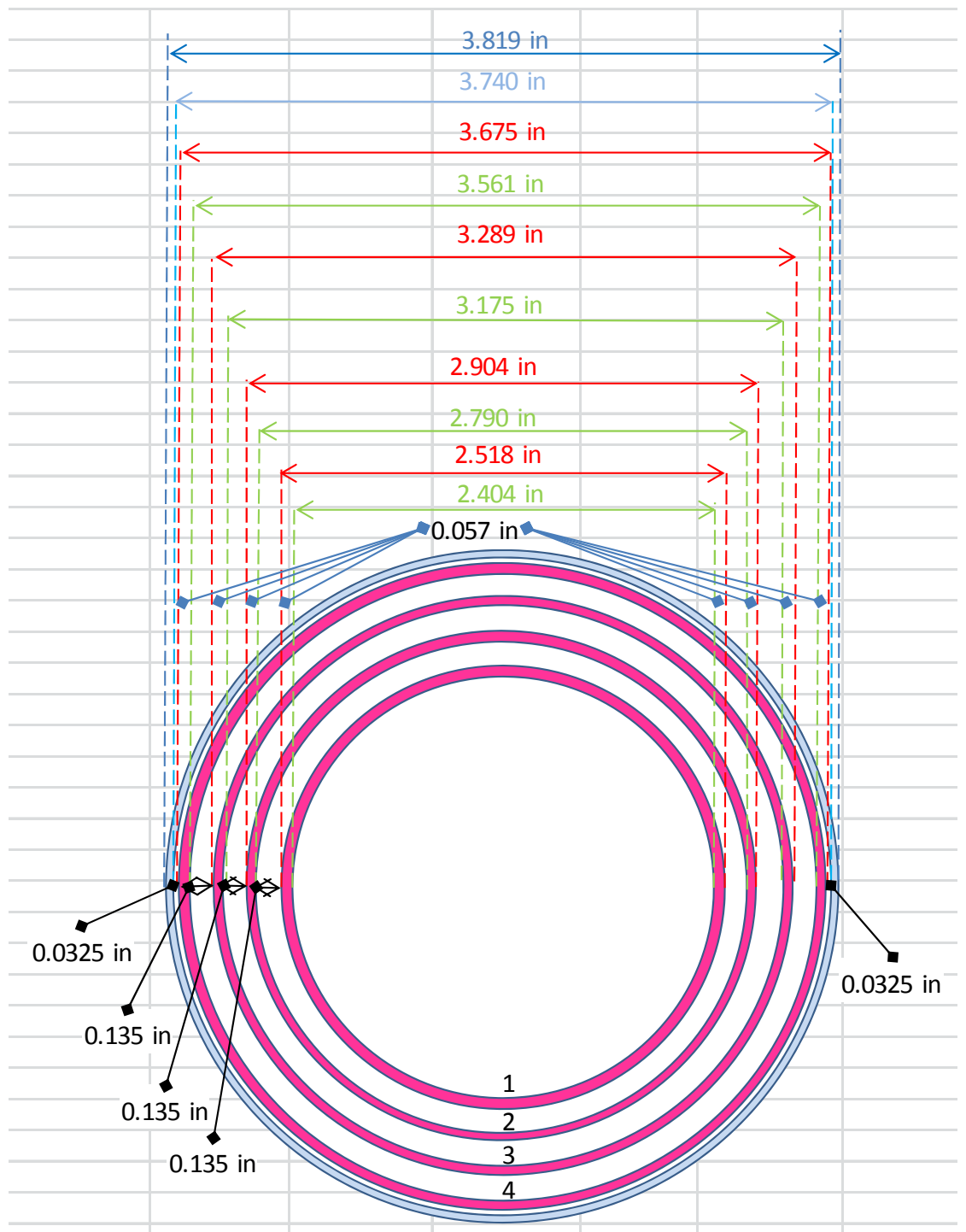

Figure A-11. Sketch of Top Cross-section of DR-3-1 Assembly 
SRNL-STI-2012-00279

Revision 1

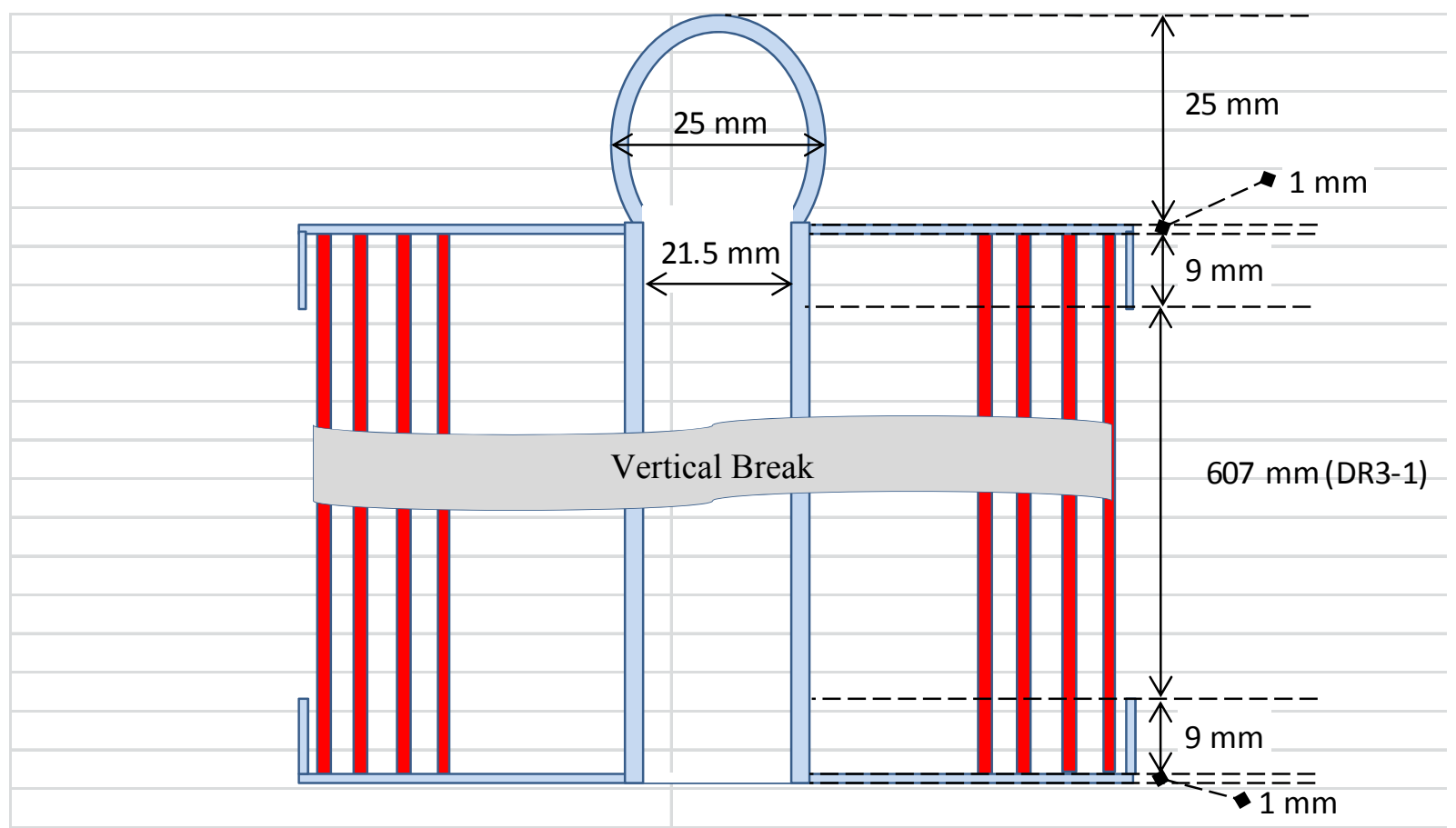

Figure A-12. Sketch of Side Cross-section of DR-3-1 Assembly

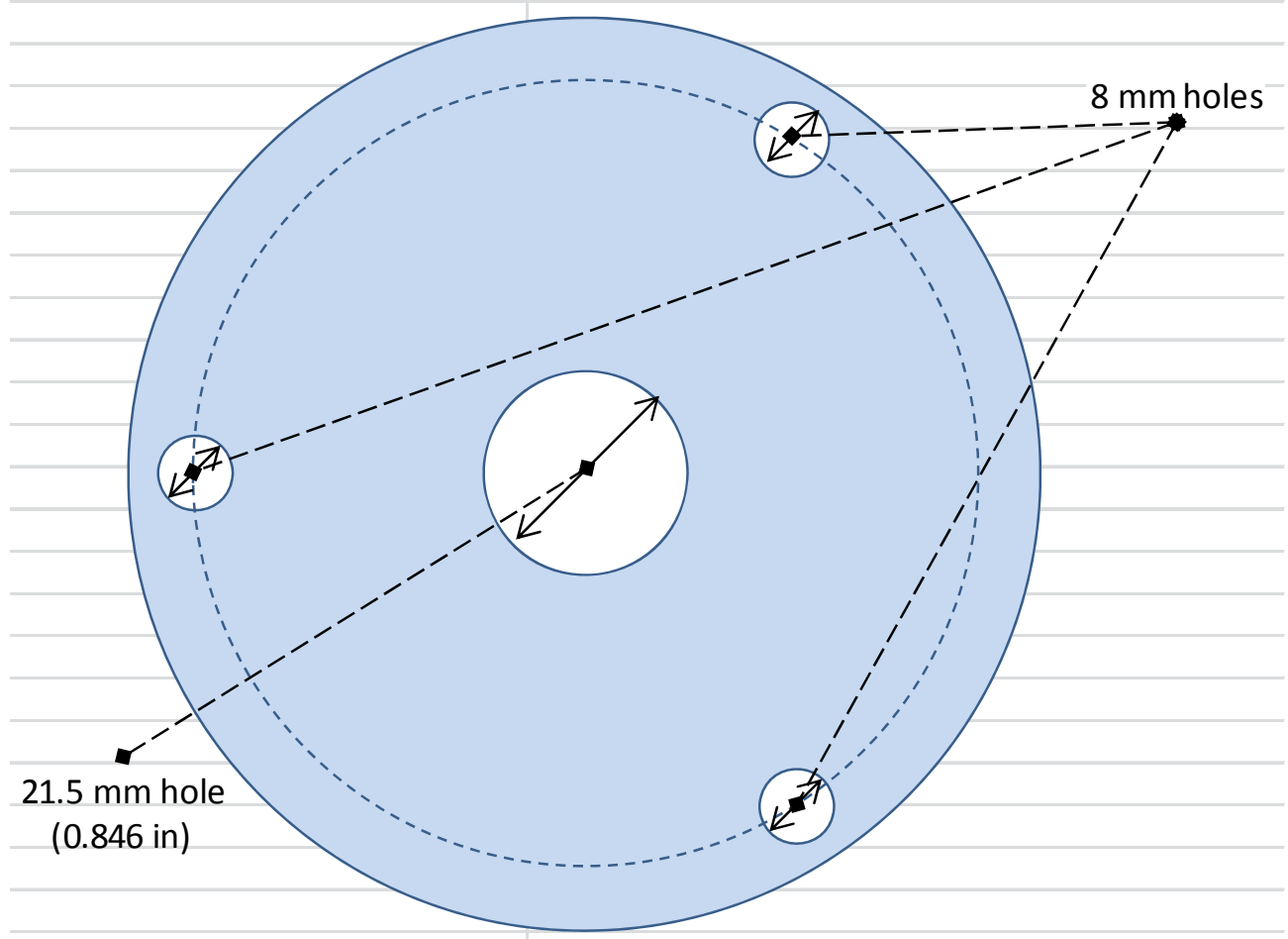

Figure A-13. Sketch of Top and Bottom Cup Plates of DR-3-1 Assembly 
SRNL-STI-2012-00279

Revision 1

Table A-12. Denmark Reactor DR-3-1 Assembly Parts and Dimensions

\begin{tabular}{|c|c|c|c|c|}
\hline DR-3-1 Part & $\begin{array}{c}\text { Outer Diameter } \\
\text { (OD) [in] }\end{array}$ & $\begin{array}{c}\text { Wall } \\
\text { [in] }\end{array}$ & $\begin{array}{c}\text { Length (vertical } \\
\text { height) [in] }\end{array}$ & Comments \\
\hline Fuel Tube 1 & 2.518 & 0.057 & 24.606 & Innermost fuel tube \\
\hline Fuel Tube 2 & 2.904 & 0.057 & 24.606 & Outside fuel tube 1 \\
\hline Fuel Tube 3 & 3.289 & 0.057 & 24.606 & Outside fuel tube 2 \\
\hline Fuel Tube 4 & 3.675 & 0.057 & 24.606 & Outermost fuel tube \\
\hline Cup Plate Assembly & 3.819 & NA & 25.669 & $\begin{array}{c}\text { Overall dimensions of cup plate } \\
\text { assembly with fuel tubes. }\end{array}$ \\
\hline Hairpin & 0.98 & 0.0787 & 52.56 & $\begin{array}{c}\text { OD for circular bent hairpin at top. } \\
\text { Length is if hairpin straightens out. }\end{array}$ \\
\hline Top and Bottom Cup Plates & 3.819 & 0.0394 & 0.39 & Length is height of cup wall. \\
\hline
\end{tabular}

$\mathrm{NA}=$ Not Applicable

Starting from the bottom of the Denmark Reactor DR-3-1 Assembly and going up vertically the exposed inner and outer surface area is calculated as shown in Table A-13. This table details the surface area calculations for specific sections of the DR-3-1 Assembly in terms of height from its bottom. The same terms and structure as defined for the L-Bundle, SRE, and HMI above are used in this table. Using the logic outlined here, the total inner and outer surface area for the DR-3-1 Assembly for the full 25.7 inch height was $513.43 \mathrm{in}^{2}$ giving an overall scaling factor for the DR-3-1 Assembly of $20.00 \mathrm{in}^{2} / \mathrm{in}$ or 1.67 $\mathrm{ft}^{2} / \mathrm{ft}$. 
Table A-13. Inner and Outer Surface Area Calculation for DR-3-1 Assembly

\begin{tabular}{|c|c|c|c|c|c|c|c|}
\hline \begin{tabular}{|c|} 
Scaling \\
Factor for \\
Height h \\
{$\left[\right.$ in $^{2} /$ in] }
\end{tabular} & $\begin{array}{l}\text { Height h } \\
\text { [in] }\end{array}$ & $\begin{array}{c}\text { Constant } \\
{\left[\text { in }^{2}\right]}\end{array}$ & $\begin{array}{c}\text { Cumulative } \\
\text { Surface } \\
\text { Area }\left[\text { in }^{2}\right]\end{array}$ & $\begin{array}{c}\text { From } \\
\text { [in] }\end{array}$ & To [in] & Description & Surface Area Sums \\
\hline 12.49 & 0.04 & 10.66 & 11.15 & 0 & 0.04 & $\begin{array}{l}\text { from bottom of bottom cup plate up to } \\
\text { start of fuel tubes (ignore inside } \\
\text { circumference area of holes since only } 1 \\
\text { mm thick and elevation change for } 40.5 \\
\text { mm circular countersunk area) }\end{array}$ & $\begin{array}{c}\text { [bottom area of cup plate }]+[\text { outer circumference } \\
\text { area of bottom cup plate }]+[\text { circumference area } \\
\text { of } 2 \text { legs of hairpin] }]=\left[\pi / 4 *\left(3.819^{2}-3 * 0.315^{2}-\right.\right. \\
\left.\left.0.846^{2}\right)\right]+\left[\pi^{*} 3.819 * \mathrm{~h}\right]+\left[\pi^{*} 0.0787 * 2 * \mathrm{~h}\right]\end{array}$ \\
\hline 20.04 & 0.35 & 15.13 & 22.23 & 0.04 & 0.39 & $\begin{array}{l}\text { from start of fuel tubes up to top of bottom } \\
\text { cup plate }\end{array}$ & $\begin{array}{c}\text { [inside face area of bottom cup plate] }+ \text { [outside } \\
\text { circumference area of bottom cup plate }]+ \\
\text { [circumference area of } 2 \text { legs of hairpin }]+[\text { inner } \\
\text { circumference area of fuel tube } 1]= \\
{\left[\left(\pi / 4 *\left(2.404^{2}-0.846^{2}\right)\right)\right]+\left[\pi^{*} 3.819 * \mathrm{~h}\right]+} \\
{\left[\pi^{*} 0.0787 * 2 * \mathrm{~h}\right]+\left[\pi^{*} 2.404 * \mathrm{~h}\right]}\end{array}$ \\
\hline 19.59 & 23.90 & 22.23 & 490.44 & 0.39 & 24.29 & $\begin{array}{c}\text { from top of bottom cup plate up to bottom } \\
\text { of top cup plate }\end{array}$ & $\begin{array}{c}\text { [circumference area of } 2 \text { legs of hairpin] }+[\text { inner } \\
\text { circumference area of fuel tube } 1]+[\text { outer } \\
\text { circumference area of fuel tube } 4]= \\
{\left[\pi^{*} 0.0787 * 2 * \mathrm{~h}\right]+\left[\pi^{*} 2.404 * \mathrm{~h}\right]+\left[\pi^{*} 3.675 * \mathrm{~h}\right]}\end{array}$ \\
\hline 20.04 & 0.35 & 490.44 & 497.54 & 24.29 & 24.65 & $\begin{array}{l}\text { from bottom of top cup plate up to top of } \\
\text { fuel tubes }\end{array}$ & $\begin{array}{c}\text { [circumference area of } 2 \text { legs of hairpin] }+[\text { inner } \\
\text { circumference area of fuel tube } 1]+[\text { outer } \\
\text { circumference area of top cup plate }]= \\
{\left[\pi^{*} 0.0787 * 2 * h\right]+\left[\pi^{*} 2.404 * \mathrm{~h}\right]+\left[\pi^{*} 3.819 * \mathrm{~h}\right]}\end{array}$ \\
\hline 12.49 & 0.04 & 501.52 & 502.01 & 24.65 & 24.69 & $\begin{array}{c}\text { from top of fuel tubes up to top of top cup } \\
\text { plate (ignore inside circumference area of } \\
\text { holes since only } 1 \mathrm{~mm} \text { thick and elevation } \\
\text { change for } 40.5 \mathrm{~mm} \text { circular countersunk } \\
\text { area) }\end{array}$ & $\begin{array}{c}\text { [inside face area of top cup plate }]+ \\
\text { [circumference area of } 2 \text { legs of hairpin }]+[\text { outer } \\
\text { circumference area of top cup plate }]= \\
{\left[\pi / 4 *\left(2.404^{2}--0.846^{2}\right)\right]+\left[\pi^{*} 3.819^{*} \mathrm{~h}\right]+} \\
{\left[\pi^{*} 0.0787 * 2 * \mathrm{~h}\right]}\end{array}$ \\
\hline 0.78 & 0.98 & 512.67 & 513.43 & 24.69 & 25.67 & $\begin{array}{l}\text { from top of top cup plate up to top of } \\
\text { hairpin (assume bent hair pin } \sim 25 \mathrm{~mm} \\
\text { Outer Diameter) }\end{array}$ & $\begin{array}{c}\text { [top area of top cup plate }]+[\text { circumference area } \\
\text { of } 2 \text { legs of hairpin curved] }=\left[\pi / 4 *\left(3.819^{2}-\right.\right. \\
\left.\left.3 * 0.315^{2}-0.846^{2}\right)\right]+\left[\pi^{*} \pi^{*} 2 / 25.4 * \mathrm{~h}\right]\end{array}$ \\
\hline
\end{tabular}




\section{A.4.2. $\quad$ DR-3-2 (Denmark Reactor) Surface Area Calculations}

A sketch of the side view of the DR-3-2 assembly is shown in Figure A-15.Figure A-14. The top cross sectional view of the DR-3-2 assembly is the same as the DR-3-1 assembly as shown in Figure A-11 showing the spacing of the concentric fuel tubes. Figure A-15 shows a cross-section side view of the DR3-2 assembly with the top and bottom cup plates holding the fuel tubes with a hairpin. The top and bottom cup plates are the same as in the DR-3-1 assembly as shown in Figure A-13. Since the cup plates are only $1 \mathrm{~mm}$ thick the inside area of holes are not counted in the surface area calculations. The elevation change in the top and bottom cup plates due to a $40.5 \mathrm{~mm}$ circular countersunk area around the center point is also ignored in the surface area calculations. The various parts of the DR-3-2 assembly have been color coded and the dimensions of these parts are shown in Table A-14.

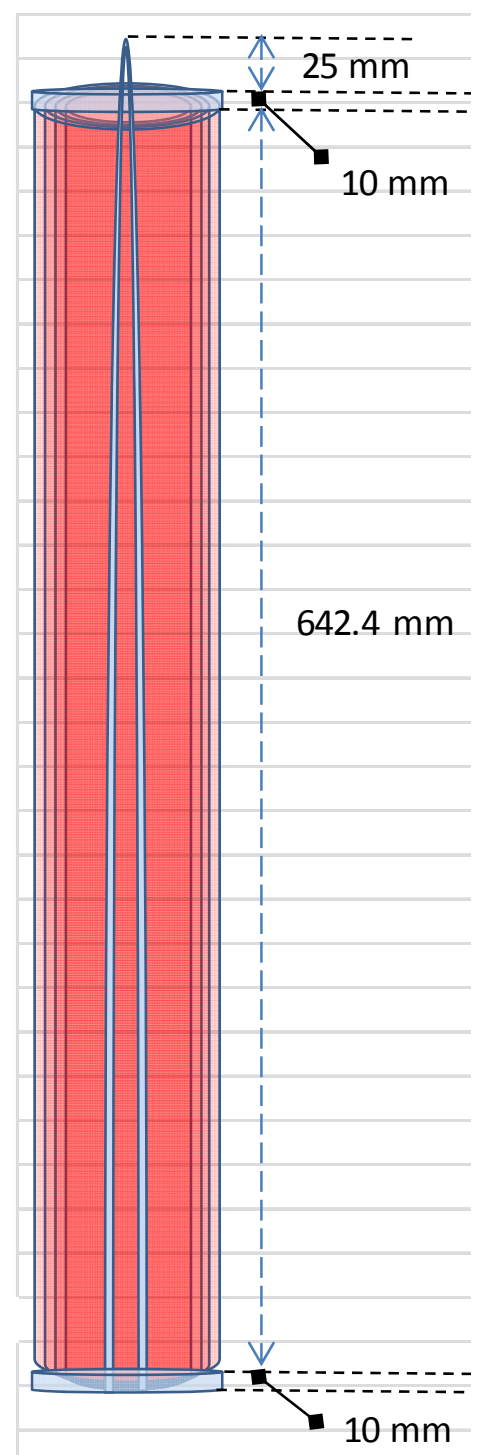

Figure A-14. Sketch of Side View of DR-3-2 Assembly 
SRNL-STI-2012-00279

Revision 1

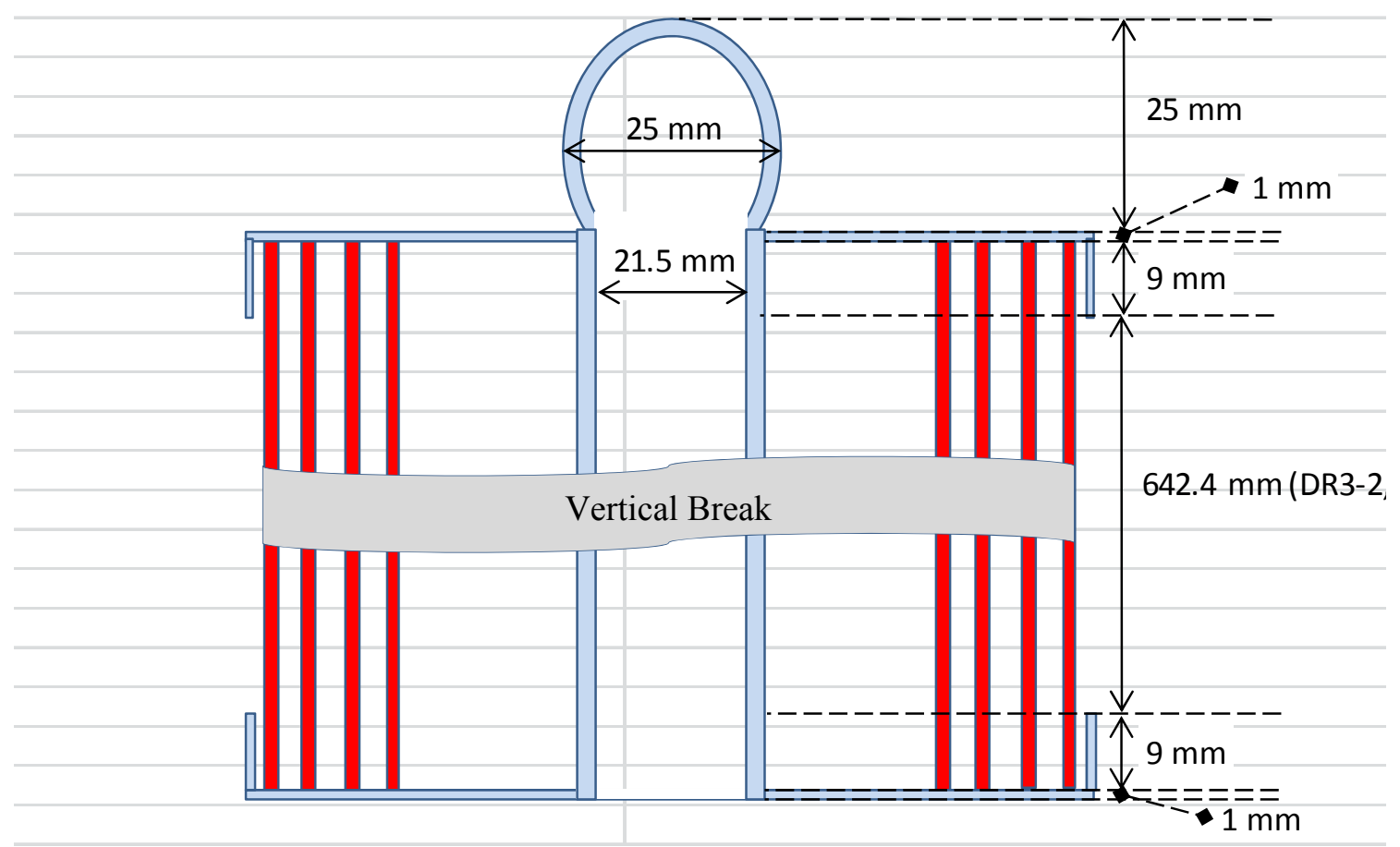

Figure A-15. Sketch of Side Cross-section of DR-3-2 Assembly

Starting from the bottom of the Denmark Reactor DR-3-2 Assembly and going up vertically the exposed inner and outer surface area is calculated as shown in Table A-14. This table details the surface area calculations for specific sections of the DR-3-2 Assembly in terms of height from its bottom. The same terms and structure as defined for the L-Bundle, SRE, and HMI above are used in this table. Using the logic outlined here, the total inner and outer surface area for the DR-3-1 Assembly for the full 27.06 inch height was $540.74 \mathrm{in}^{2}$ giving an overall scaling factor for the DR-3-1 Assembly of $19.98 \mathrm{in}^{2} /$ in or 1.67 $\mathrm{ft}^{2} / \mathrm{ft}$. 
Table A-14. Inner and Outer Surface Area Calculation for DR-3-2 Assembly

\begin{tabular}{|c|c|c|c|c|c|c|c|}
\hline \begin{tabular}{|c} 
Scaling \\
Factor \\
for \\
Height \\
$\mathbf{h}$ \\
{$\left[\mathrm{in}^{2} / \mathrm{in}\right]$} \\
\end{tabular} & $\begin{array}{c}\text { Height } \\
\text { h [in] }\end{array}$ & $\begin{array}{c}\text { Constant } \\
{\left[\mathrm{in}^{2}\right]}\end{array}$ & $\begin{array}{l}\text { Cumulative } \\
\text { Surface } \\
\text { Area }\left[i^{2}\right] \\
\end{array}$ & $\begin{array}{c}\text { From } \\
\text { [in] }\end{array}$ & To [in] & Description & Surface Area Sums \\
\hline 12.49 & 0.04 & 10.66 & 11.15 & 0.00 & 0.04 & $\begin{array}{c}\text { from bottom of } \\
\text { bottom cup plate up } \\
\text { to start of fuel tubes } \\
\text { (ignore inside } \\
\text { circumference area } \\
\text { of holes since only } \\
1 \mathrm{~mm} \text { thick and } \\
\text { elevation change } \\
\text { for } 40.5 \mathrm{~mm} \\
\text { circular } \\
\text { countersunk area) }\end{array}$ & $\begin{array}{c}\text { [bottom area of cup plate] }+ \\
\text { [outer circumference area of } \\
\text { bottom cup plate] }+ \\
\text { [circumference area of } 2 \text { legs } \\
\text { of hairpin }]=\left[\pi / 4 *\left(3.819^{2}-\right.\right. \\
\left.\left.3 * 0.315^{2}-0.846^{2}\right)\right]+ \\
{\left[\pi^{*} 3.819^{*} \mathrm{~h}\right]+} \\
{\left[\pi^{*} 0.0787 * 2 * \mathrm{~h}\right]}\end{array}$ \\
\hline 20.04 & 0.35 & 15.13 & 22.23 & 0.04 & 0.39 & $\begin{array}{l}\text { from start of fuel } \\
\text { tubes up to top of } \\
\text { bottom cup plate }\end{array}$ & $\begin{array}{c}\text { [inside face area of bottom } \\
\text { cup plate] }+ \text { [outside } \\
\text { circumference area of bottom } \\
\text { cup plate }]+[\text { circumference } \\
\text { area of } 2 \text { legs of hairpin] }+ \\
\text { [inner circumference area of } \\
\text { fuel tube } 1]=\left[\left(\pi / 4 *\left(2.404^{2}-\right.\right.\right. \\
\left.\left.\left.0.846^{2}\right)\right)\right]+\left[\pi^{*} 3.819 * \mathrm{~h}\right]+ \\
{\left[\pi^{*} 0.0787 * 2 * \mathrm{~h}\right]+\left[\pi^{*} 2.404 * \mathrm{~h}\right]}\end{array}$ \\
\hline 19.59 & 25.29 & 22.23 & 517.74 & 0.39 & 25.69 & $\begin{array}{c}\text { from top of bottom } \\
\text { cup plate up to } \\
\text { bottom of top cup } \\
\text { plate }\end{array}$ & $\begin{array}{c}\text { [circumference area of } 2 \text { legs } \\
\text { of hairpin] + [inner } \\
\text { circumference area of fuel } \\
\text { tube } 1]+ \text { [outer circumference } \\
\text { area of fuel tube } 4]= \\
{\left[\pi^{*} 0.0787 * 2 * \mathrm{~h}\right]+} \\
{\left[\pi^{*} 2.404 * \mathrm{~h}\right]+\left[\pi^{*} 3.675^{*} \mathrm{~h}\right]}\end{array}$ \\
\hline 20.04 & 0.35 & 517.74 & 524.85 & 25.69 & 26.04 & $\begin{array}{c}\text { from bottom of top } \\
\text { cup plate up to top } \\
\text { of fuel tubes }\end{array}$ & $\begin{array}{c}\text { [circumference area of } 2 \text { legs } \\
\text { of hairpin] }+ \text { [inner } \\
\text { circumference area of fuel } \\
\text { tube } 1]+ \text { [outer circumference } \\
\text { area of top cup plate }= \\
{\left[\pi^{*} 0.0787 * 2 * \mathrm{~h}\right]+} \\
{\left[\pi^{*} 2.404 * \mathrm{~h}\right]+\left[\pi^{*} 3.819 * \mathrm{~h}\right]}\end{array}$ \\
\hline 12.49 & 0.04 & 528.82 & 529.31 & 26.04 & 26.08 & \begin{tabular}{|} 
from top of fuel \\
tubes up to top of \\
top cup plate \\
(ignore inside \\
circumference area \\
of holes since only \\
$1 \mathrm{~mm}$ thick and \\
elevation change \\
for $40.5 \mathrm{~mm}$ \\
circular \\
countersunk area)
\end{tabular} & $\begin{array}{c}\text { [inside face area of top cup } \\
\text { plate }]+[\text { circumference area } \\
\text { of } 2 \text { legs of hairpin }]+ \text { [outer } \\
\text { circumference area of top cup } \\
\text { plate }]=\left[\pi / 4 *\left(2.404^{2}--0.846^{2}\right)\right] \\
+\left[\pi^{*} 3.819 * \mathrm{~h}\right]+ \\
{\left[\pi^{*} 0.0787 * 2 * \mathrm{~h}\right]}\end{array}$ \\
\hline
\end{tabular}


Table A-14. Inner and Outer Surface Area Calculation for DR-3-2 Assembly

\begin{tabular}{|c|c|c|c|c|c|c|c|}
\hline \begin{tabular}{|l} 
Scaling \\
Factor \\
for \\
Height \\
h \\
{$\left[\right.$ in $^{2} /$ in $]$} \\
\end{tabular} & $\begin{array}{c}\text { Height } \\
h \text { [in] }\end{array}$ & $\begin{array}{c}\text { Constant } \\
{\left[\text { in }^{2}\right]}\end{array}$ & $\begin{array}{c}\text { Cumulative } \\
\text { Surface } \\
\left.\text { Area [in }{ }^{2}\right] \\
\end{array}$ & $\begin{array}{c}\text { From } \\
\text { [in] }\end{array}$ & To [in] & Description & Surface Area Sums \\
\hline 0.78 & 0.98 & 539.97 & 540.74 & 26.08 & 27.06 & $\begin{array}{c}\text { from top of top cup } \\
\text { plate up to top of } \\
\text { hairpin (assume } \\
\text { bent hair pin } \sim 25 \\
\text { mm Outer } \\
\text { Diameter) }\end{array}$ & $\begin{array}{c}\text { [top area of top cup plate] }+ \\
\text { [circumference area of } 2 \text { legs } \\
\text { of hairpin curved] }= \\
{\left[\pi / 4 *\left(3.819^{2}-3 * 0.315^{2}-\right.\right.} \\
\left.\left.0.846^{2}\right)\right]+\left[\pi^{*} \pi^{*} 2 / 25.4 * \mathrm{~h}\right]\end{array}$ \\
\hline
\end{tabular}




\section{Distribution:}

K. M. Fox, 999-W

S. D. Fink, 773-A

B. J. Giddings, 786-5A

C. C. Herman, 999-W

S. L. Marra, 773-A

F. M. Pennebaker, 773-42A

Records Administration (EDWS)

E. A. Kyser, 773-A

T. C. Shehee, 773-A

M. C. Thompson, 773-A

W. E. Daniel, 999-W

E. K. Hansen, 999-W

S. A. Thomas, 703-46A
W. E. Harris, 704-2H

J. B. Schaade, 704-2H

P. B. Andrews, 704-2H

W. G. Dyer, 704-2H

K. A. Dukes, 221-H

W. H. Clifton, 704-2H

S. L. Hudlow, 221-H

J. L. Bodkin, 221-H

R. G. Brown, 221-H

T. L. Tice, $221-\mathrm{H}$

J. R. Lint, 221-H

B. M. Williamson, 221-H

J. A. Schlesser, 707-C

M. C. Chandler, 703-H

V. E. Magoulas, 703-H

M. J. Lewczyk, 221-H

B. L. Sims, 221-H

S. O. Reed, 221-H 